\title{
Synthesis of Carbazoles and Carbazole-containing Heterocycles via Rhodium-Catalyzed Tandem Carbonylative Benzannulations
}

\author{
Wangze Song, ${ }^{\dagger}$ Xiaoxun Li, ${ }^{\dagger}$ Ka Yang, ${ }^{\dagger}$ Xian-liang Zhao, ${ }^{\dagger}$ Daniel A. Glazier, ${ }^{\star}$ Bao- \\ $\min \mathrm{Xi}^{\S} *$ and Weiping Tang ${ }^{\dagger, *, *}$ \\ $\uparrow$ School of Pharmacy, University of Wisconsin-Madison, Madison, 53705, United States \\ ${ }^{\S}$ Guangdong Provincial Key Laboratory of New Drug Screening, School of Pharmaceutical Sciences, \\ Southern Medical University, Guangzhou, Guangdong Province 510515, PR China \\ $¥$ Department of Chemistry, University of Wisconsin-Madison, Madison, 53706, United States
}




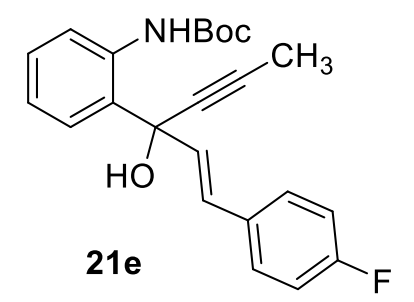

$400 \mathrm{MHz}, \mathrm{CDCl}_{3}$

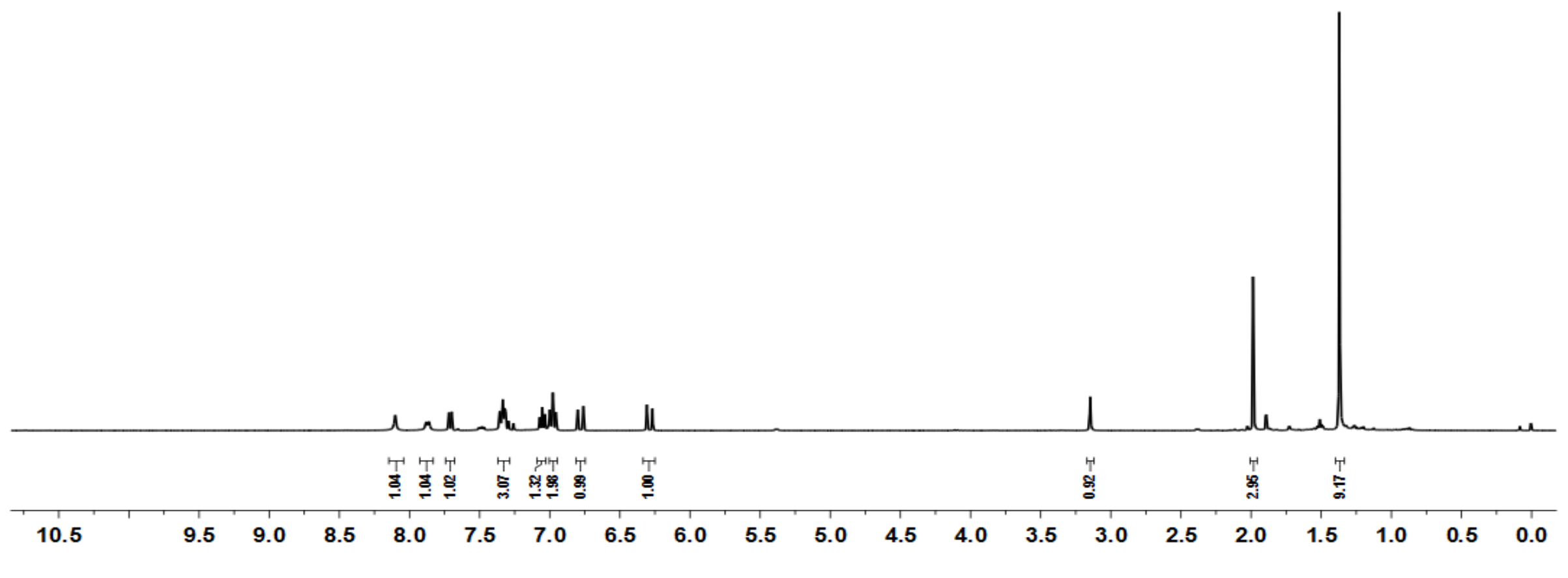




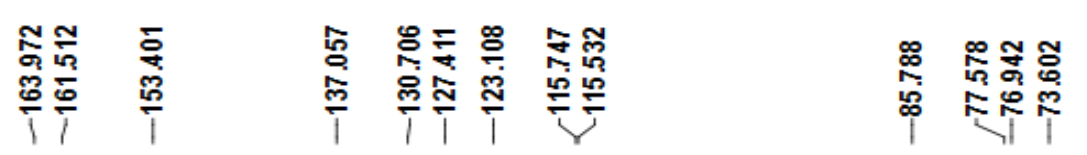

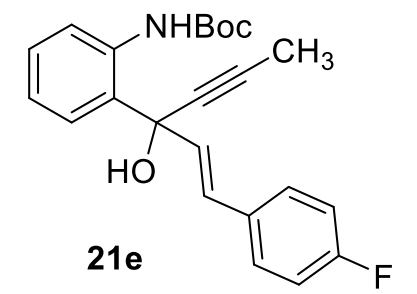

$100 \mathrm{MHz}, \mathrm{CDCl}_{3}$

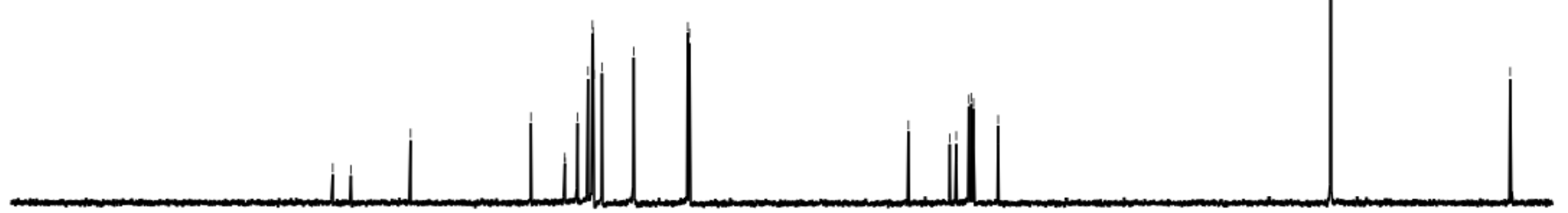



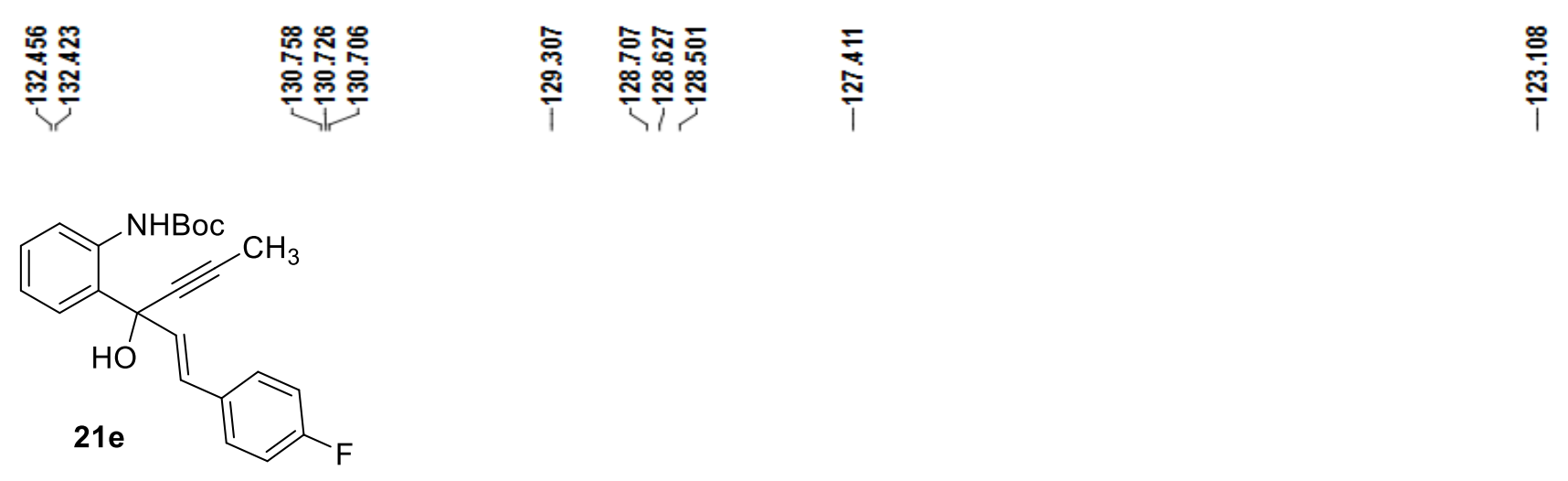

$100 \mathrm{MHz}, \mathrm{CDCl}_{3}$

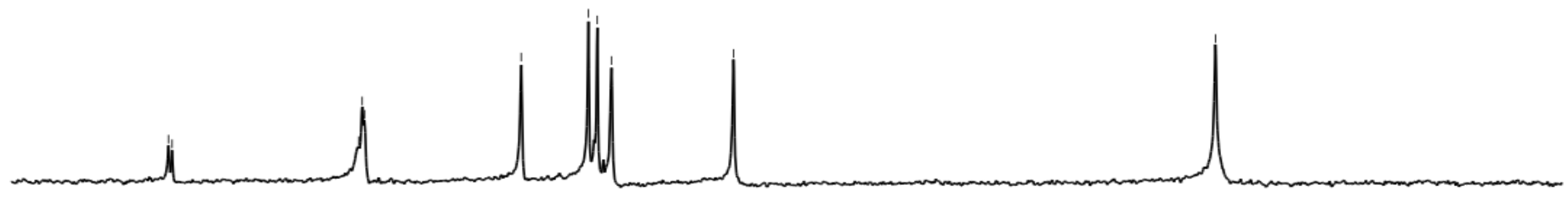




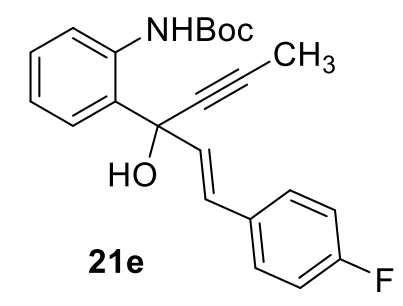

$100 \mathrm{MHz}, \mathrm{CDCl}_{3}$

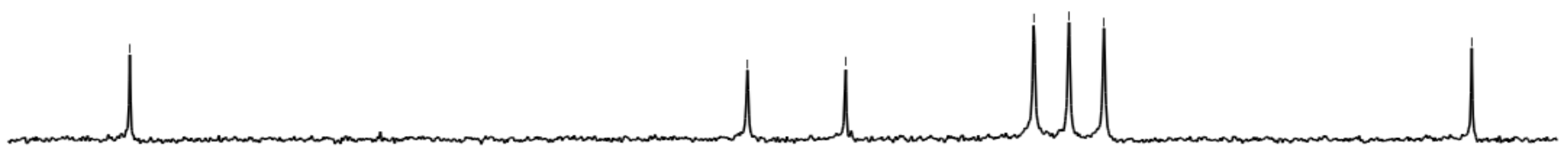




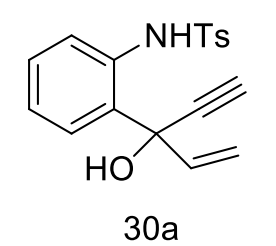

$500 \mathrm{MHz}, \mathrm{CDCl} 3$

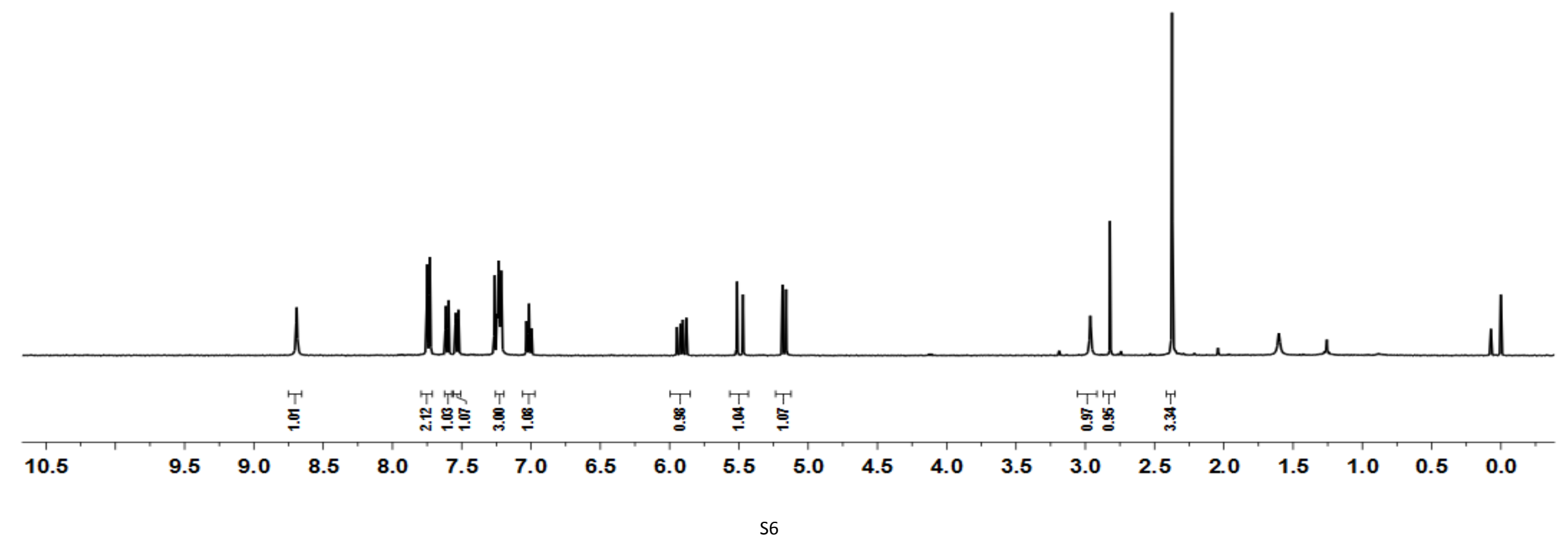



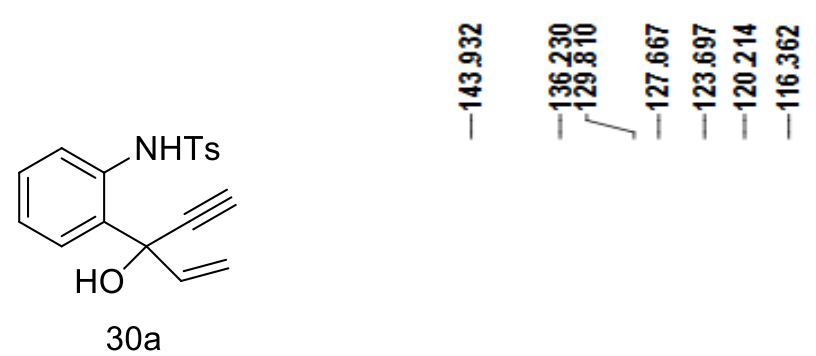

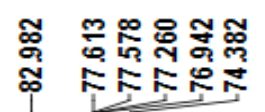

$125 \mathrm{MHz}, \mathrm{CDCl} 3$

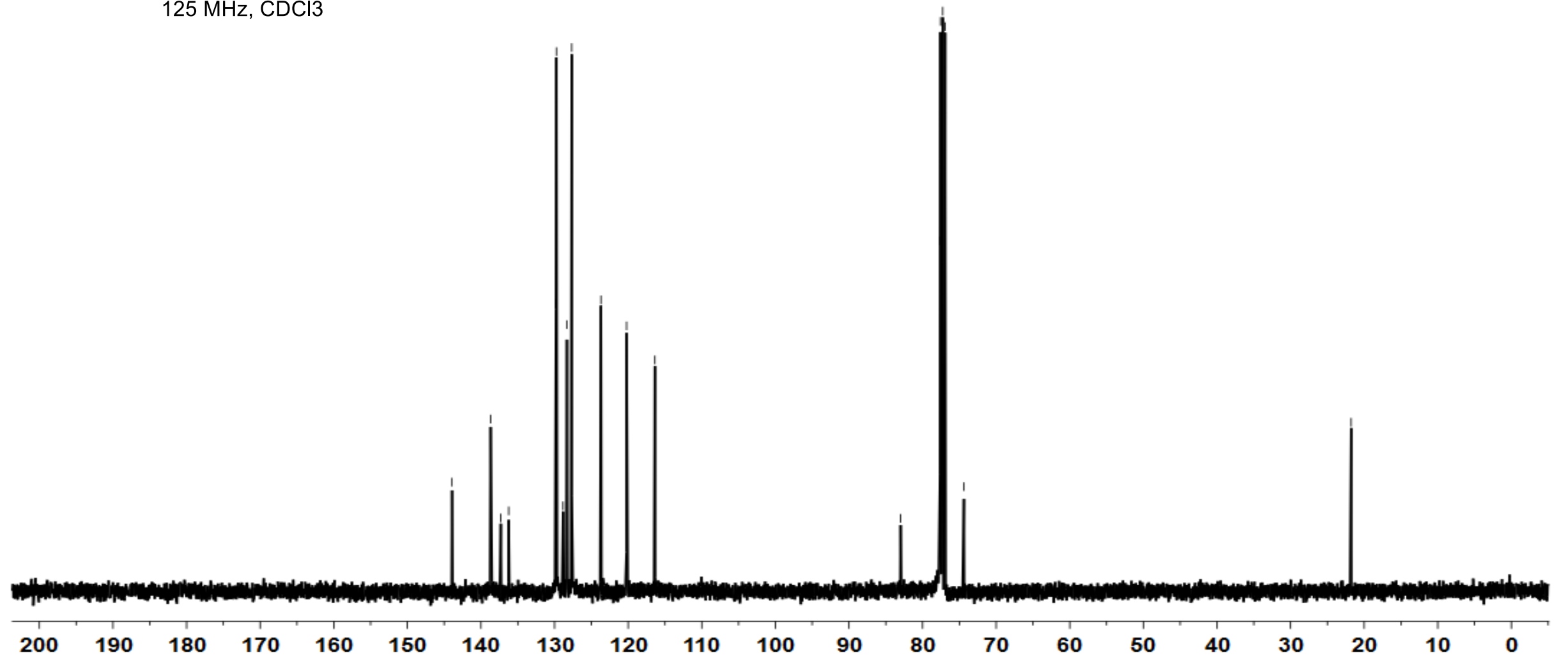




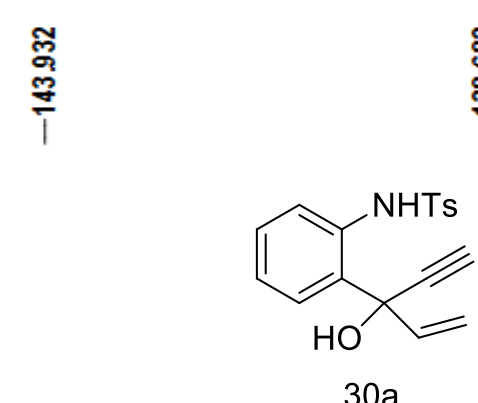

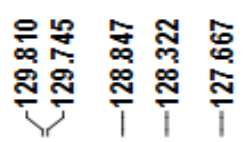

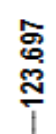

$\underset{\frac{1}{4}}{\stackrel{1}{4}}$

:

$30 a$

$125 \mathrm{MHz}, \mathrm{CDCl} 3$

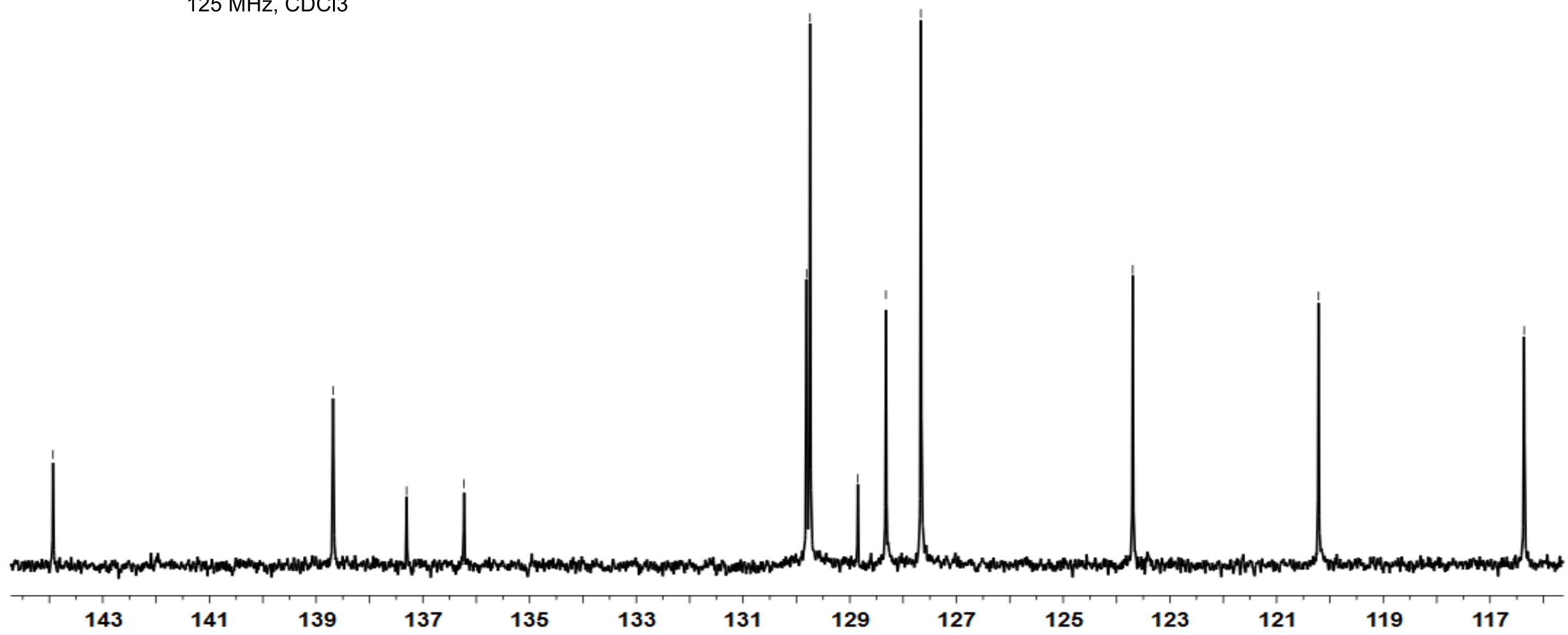




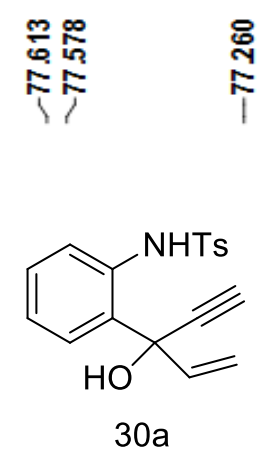

$125 \mathrm{MHz}, \mathrm{CDCl} 3$

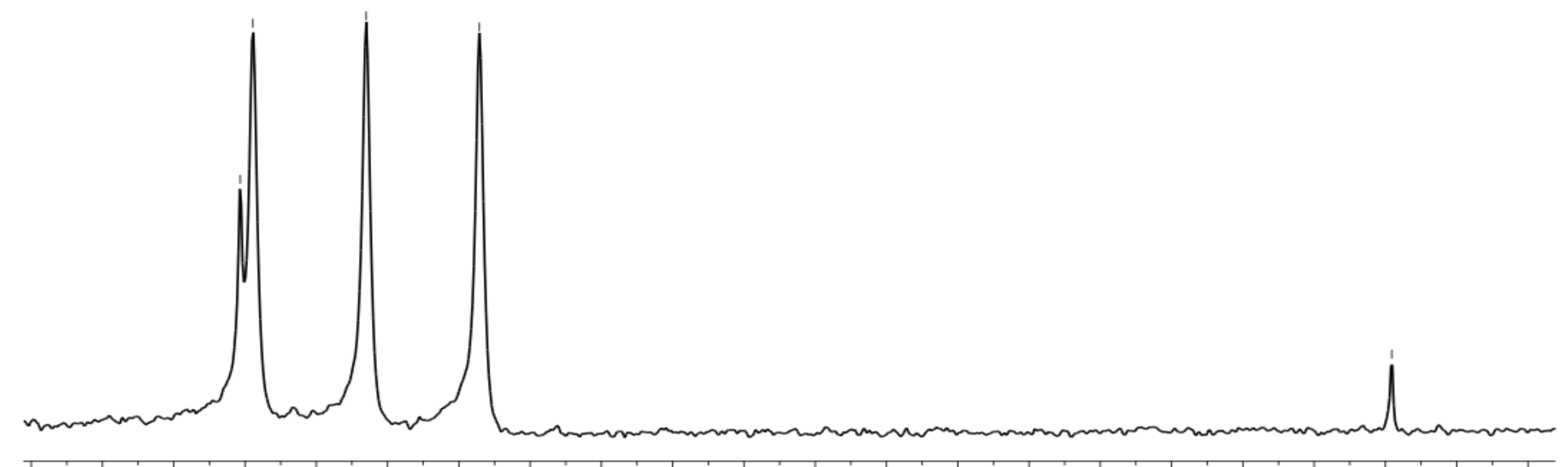

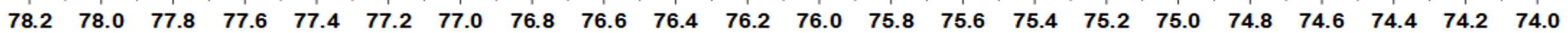



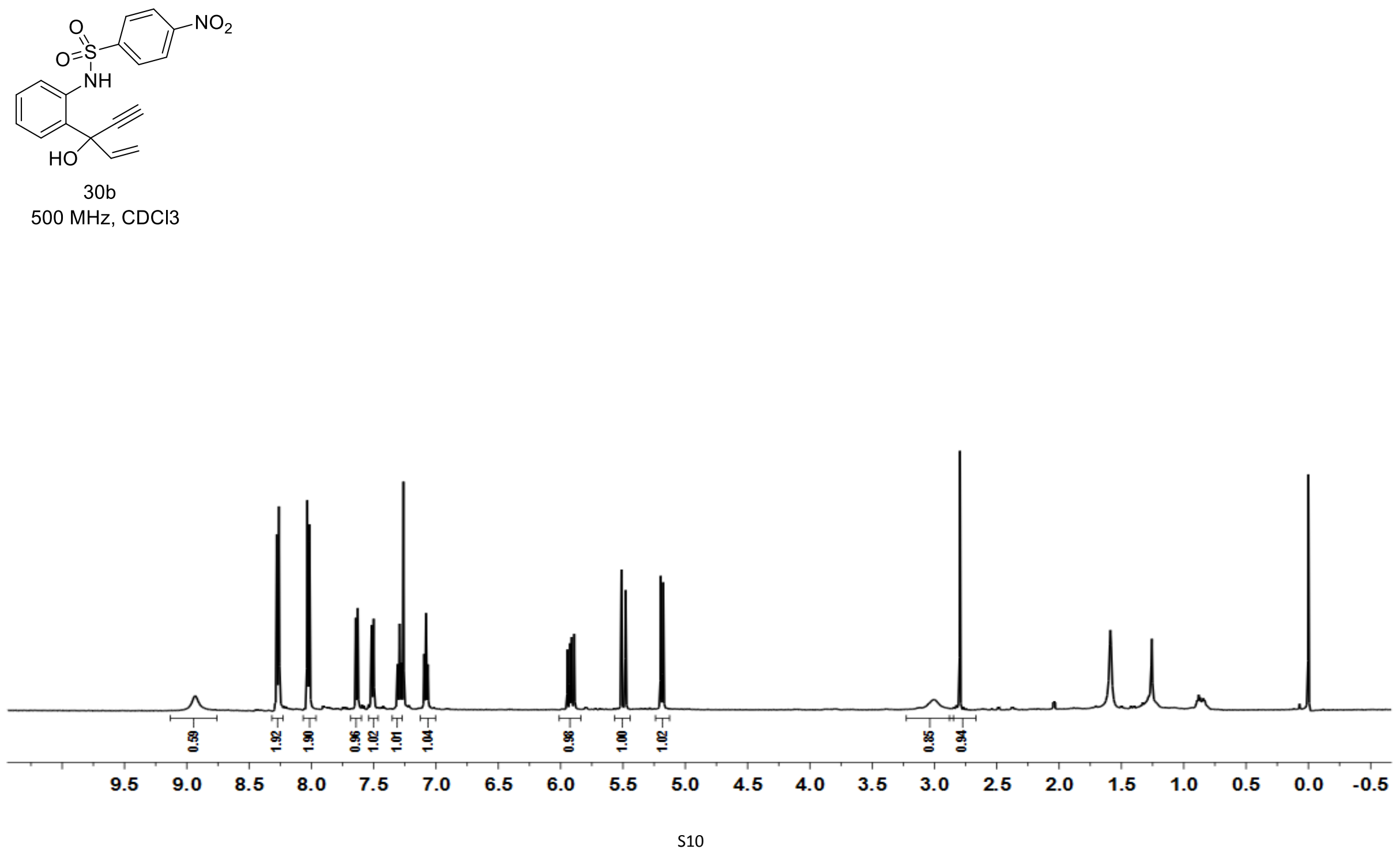


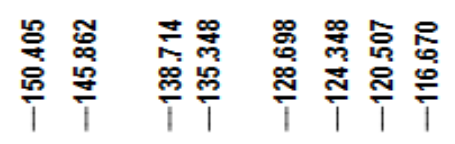

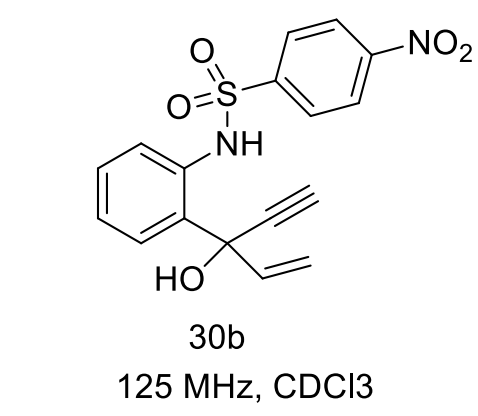

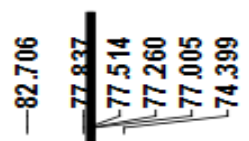

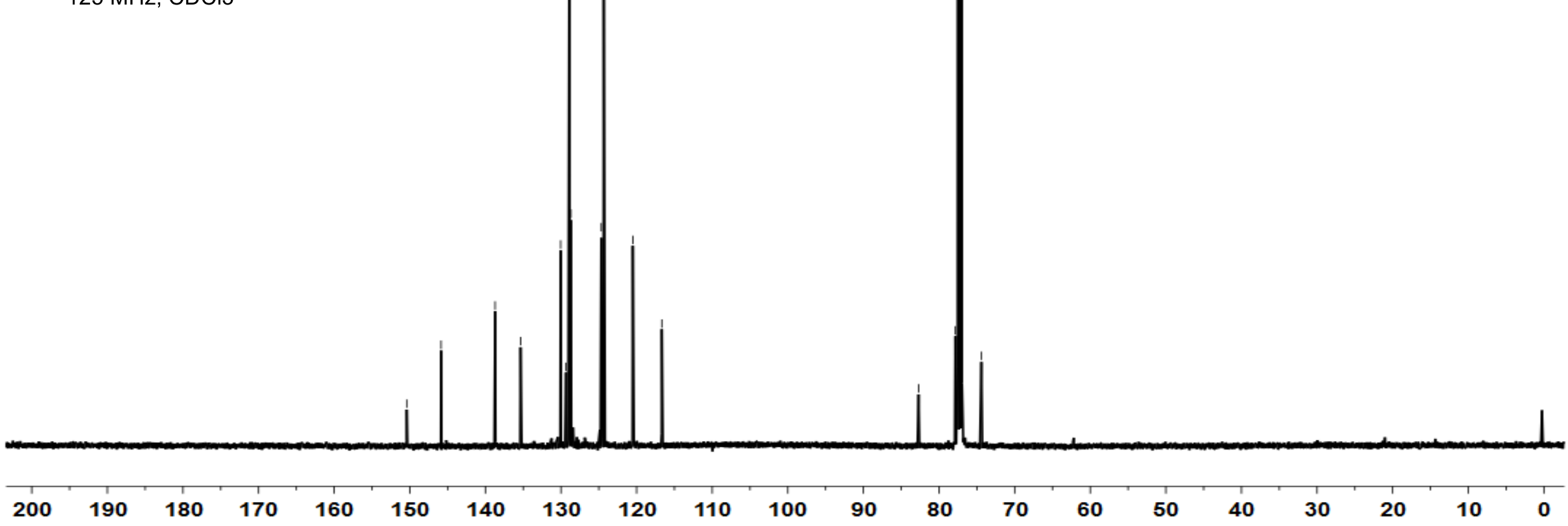

S11 
$\underset{\substack{1 \\ \frac{1}{1}}}{\frac{5}{10}}$

商

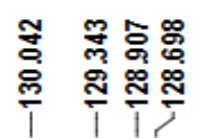

站

సิ

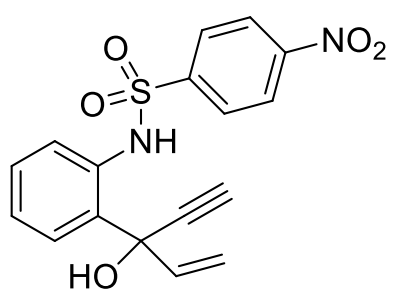

$30 \mathrm{~b}$

$125 \mathrm{MHz}, \mathrm{CDCl} 3$

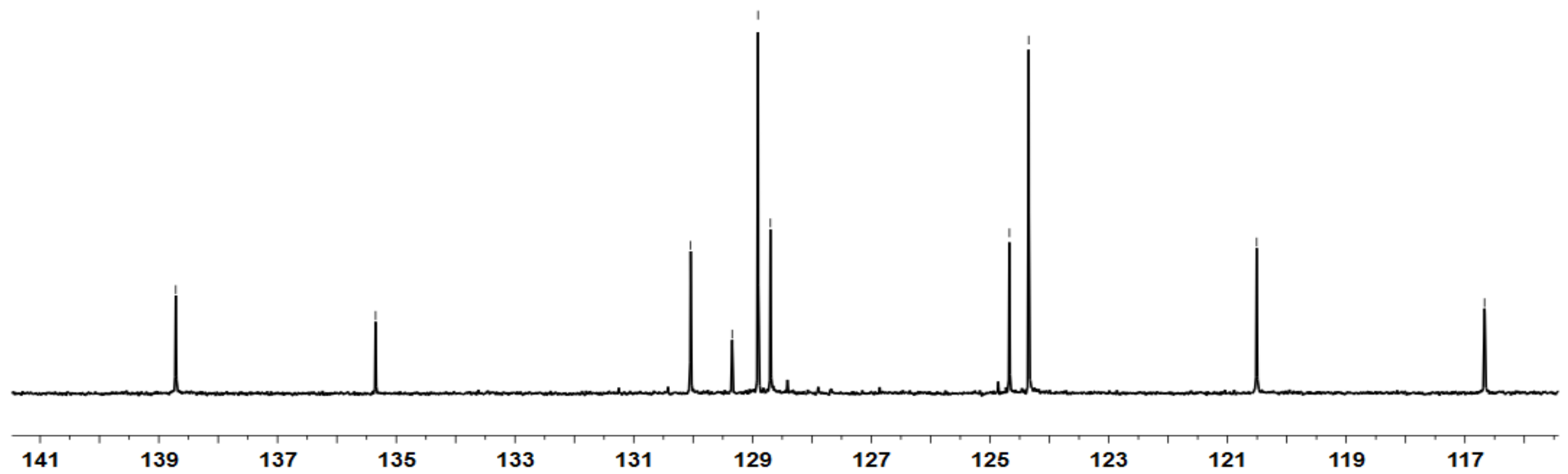




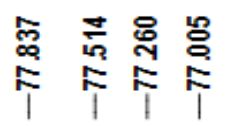

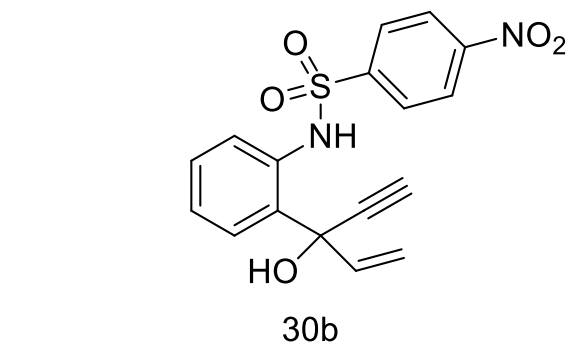

$125 \mathrm{MHz}, \mathrm{CDCl} 3$

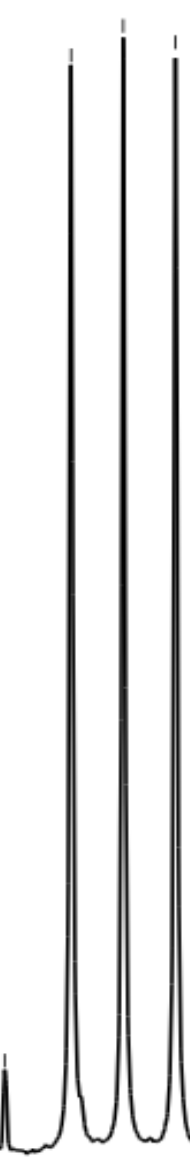

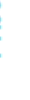

\author{
$125 \mathrm{MHZ}, \mathrm{CDCl}$
}
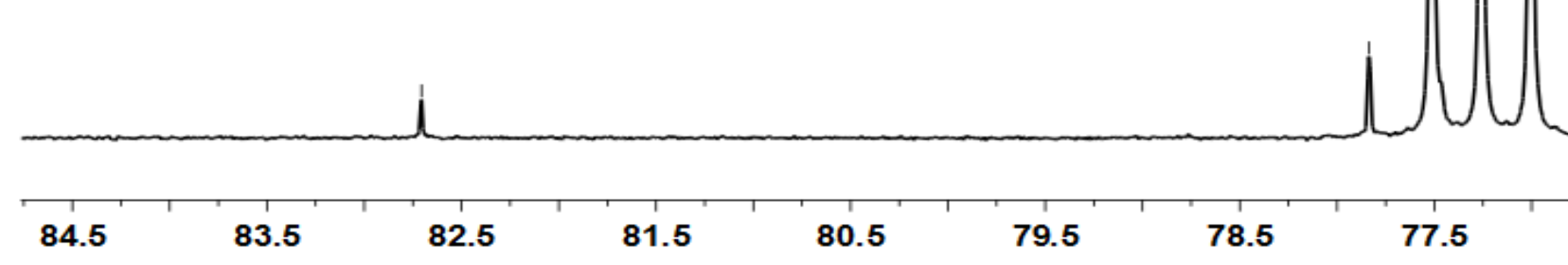

78.5

77.5

76.5

75.5

74.5 73.5 


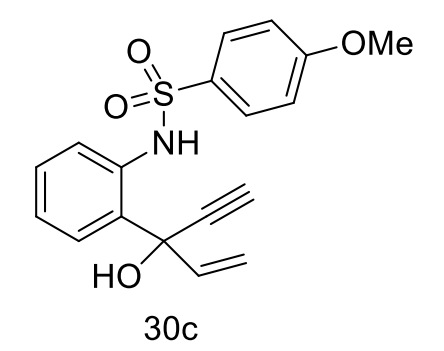

$500 \mathrm{MHz}, \mathrm{CDCl} 3$

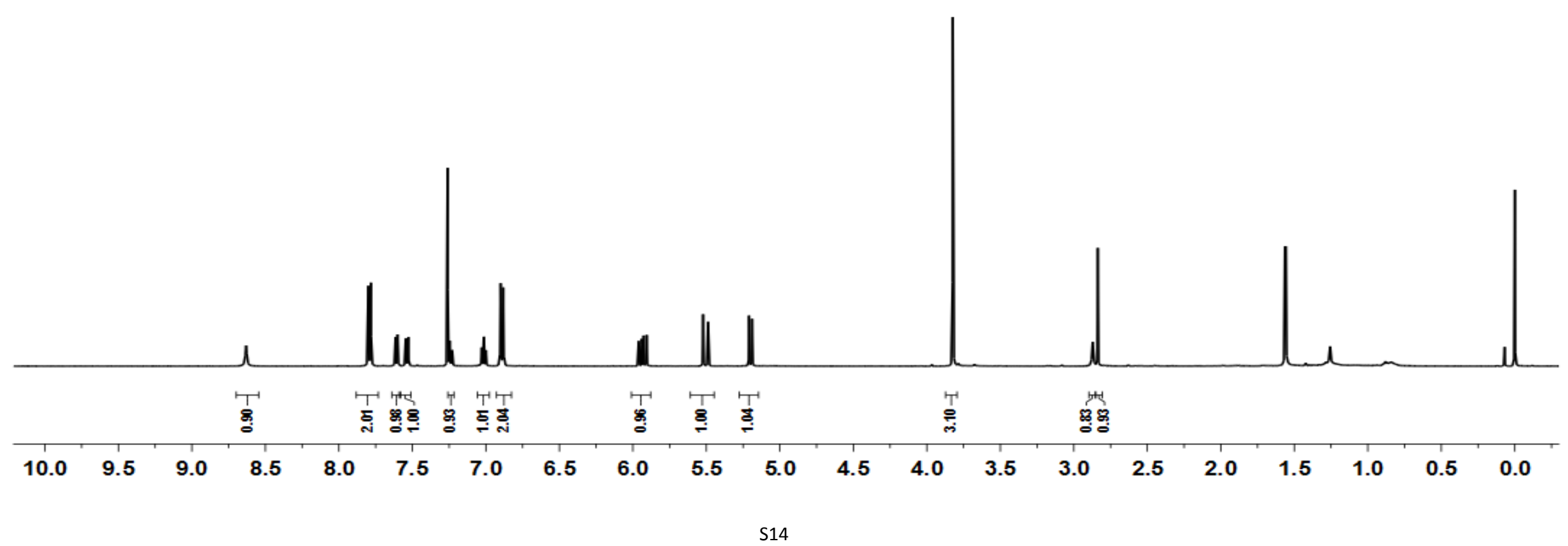




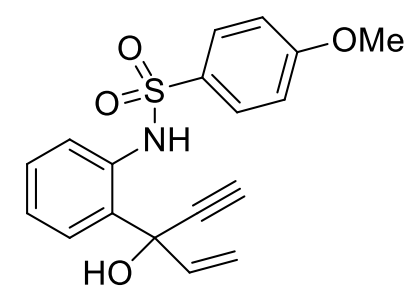

$30 \mathrm{c}$

$125 \mathrm{MHz}, \mathrm{CDCl} 3$

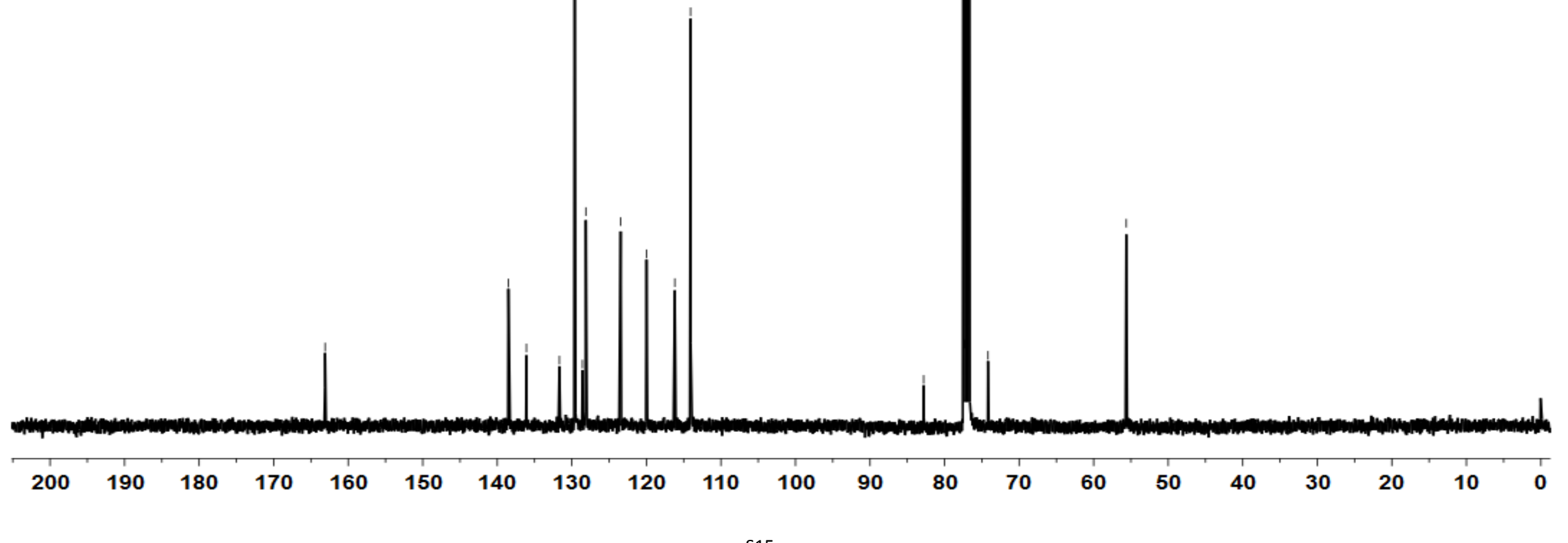




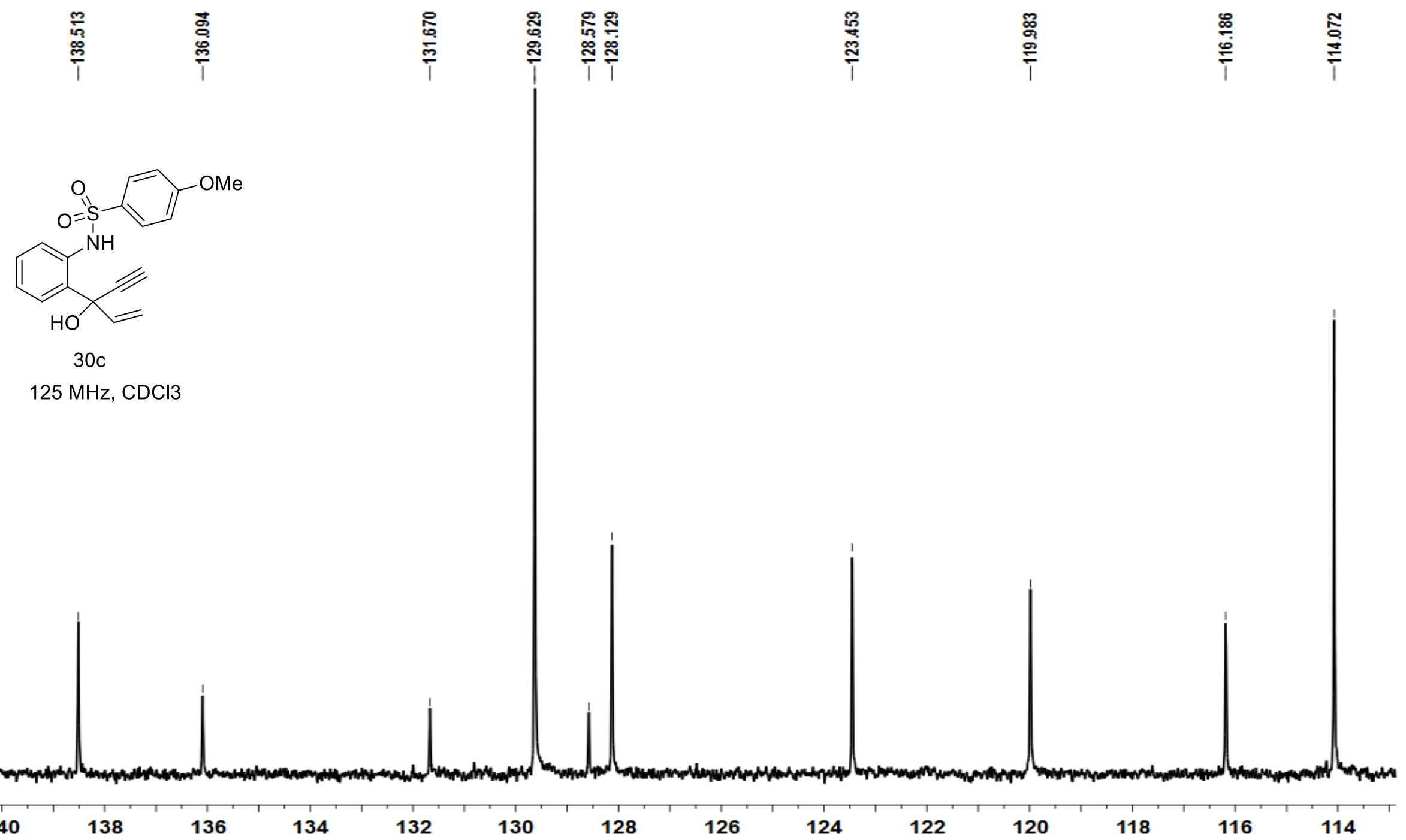




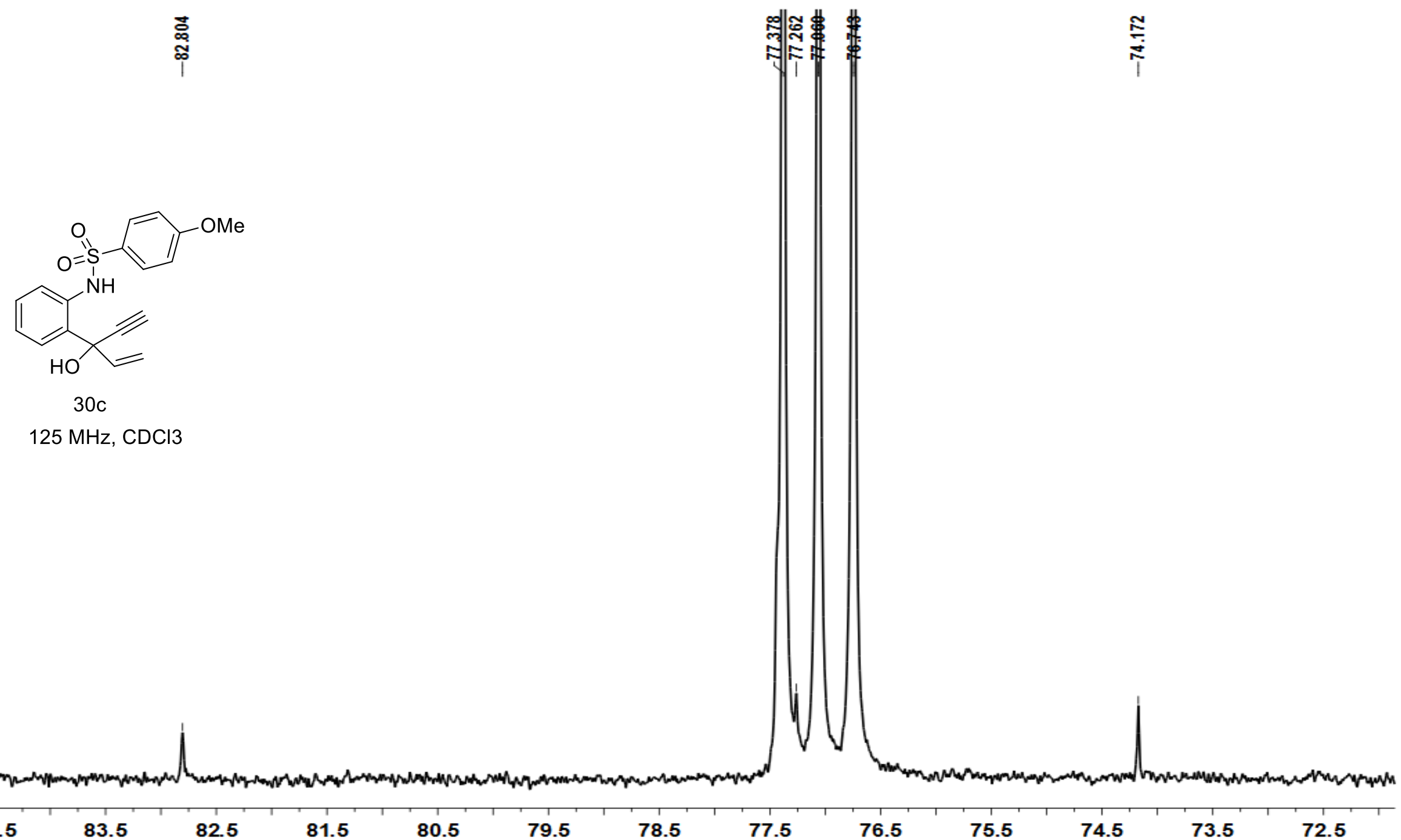




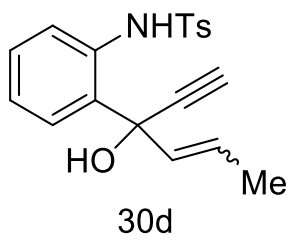

$400 \mathrm{MHz}, \mathrm{CDCl} 3$

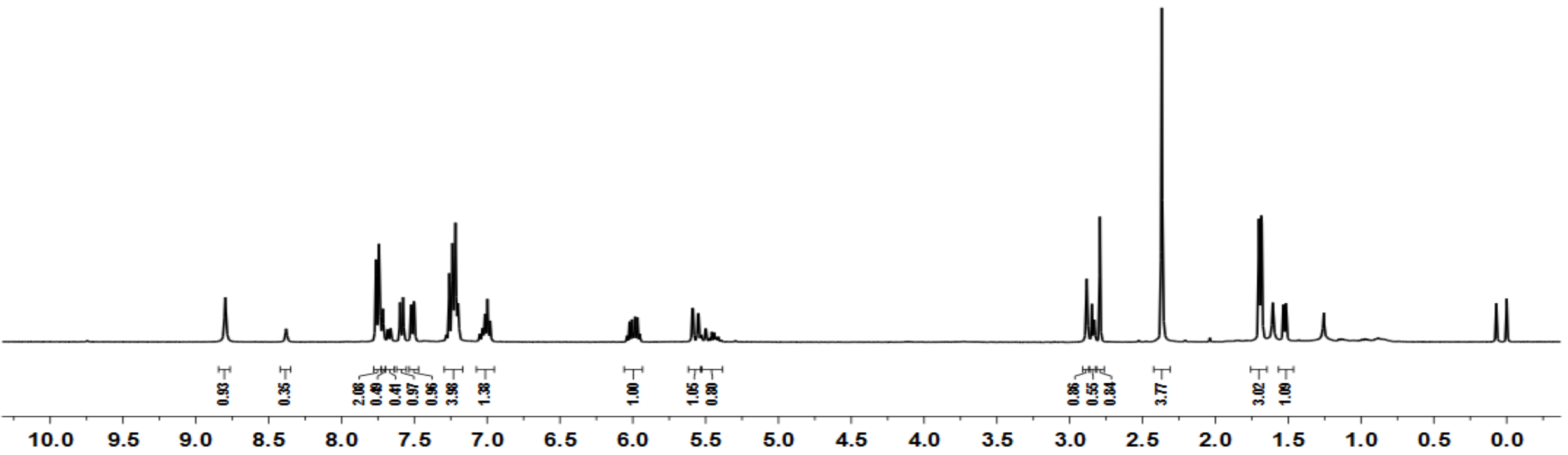

S18 


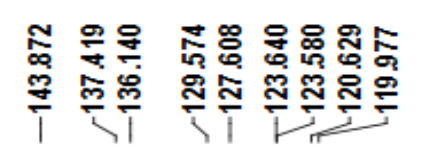

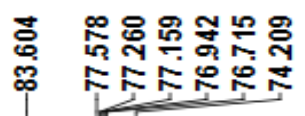

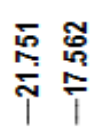

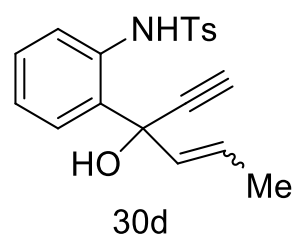

$125 \mathrm{MHz}, \mathrm{CDCl} 3$

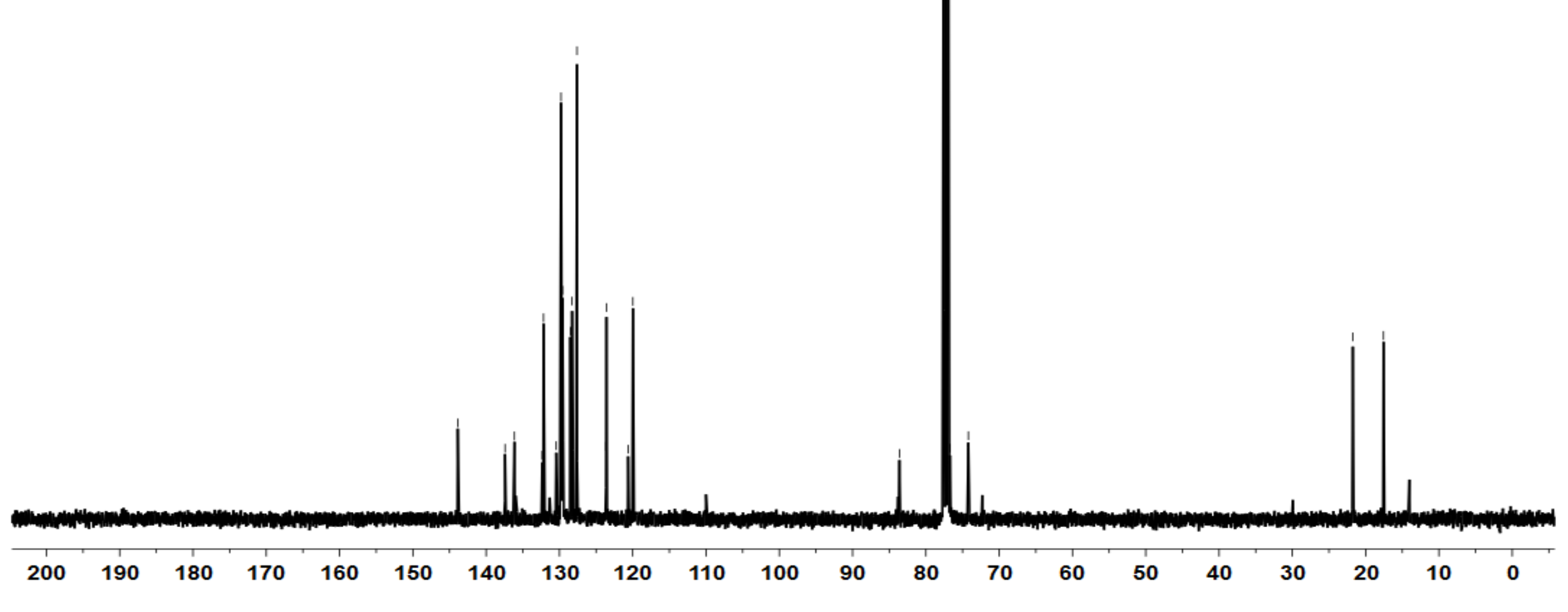




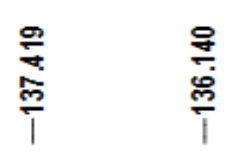

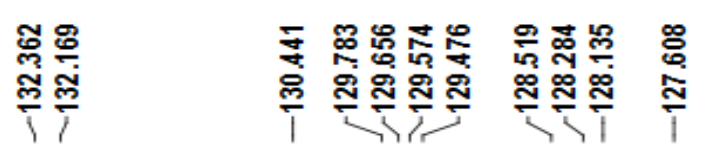

윯

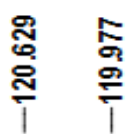

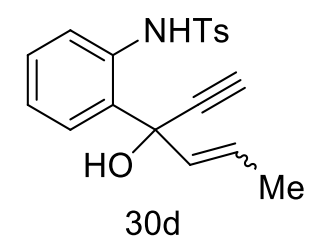

$125 \mathrm{MHz}, \mathrm{CDCl} 3$

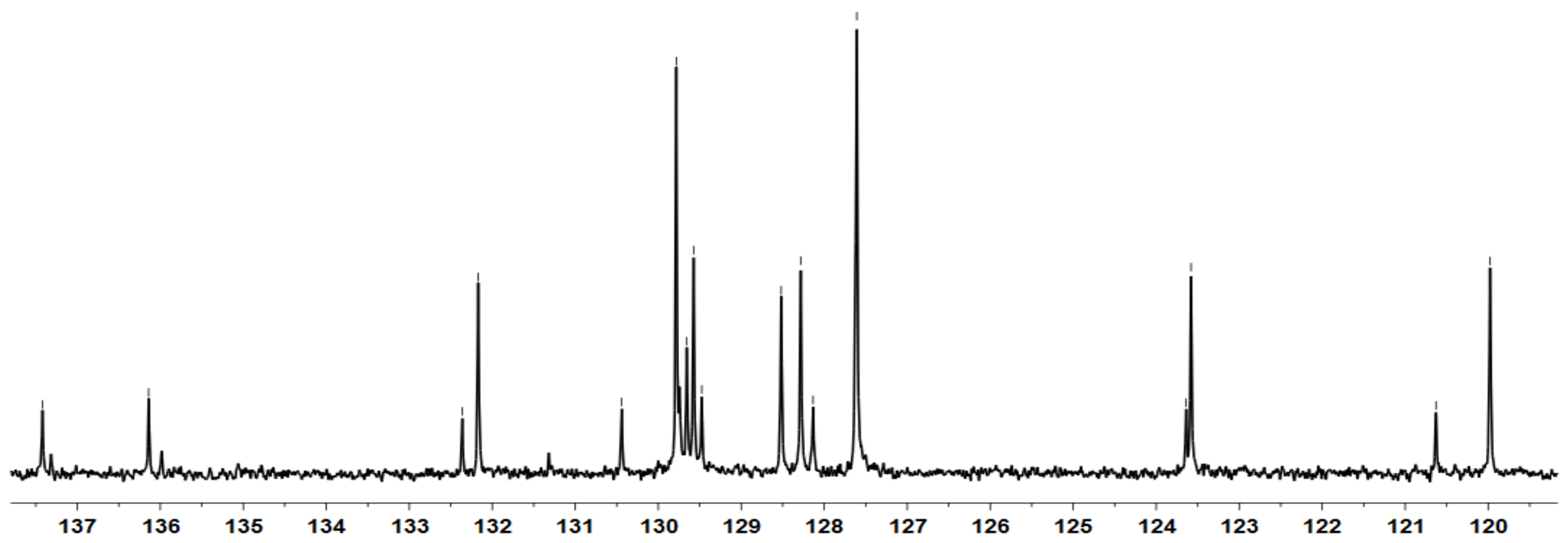




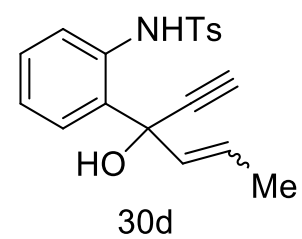

$125 \mathrm{MHz}, \mathrm{CDCl} 3$

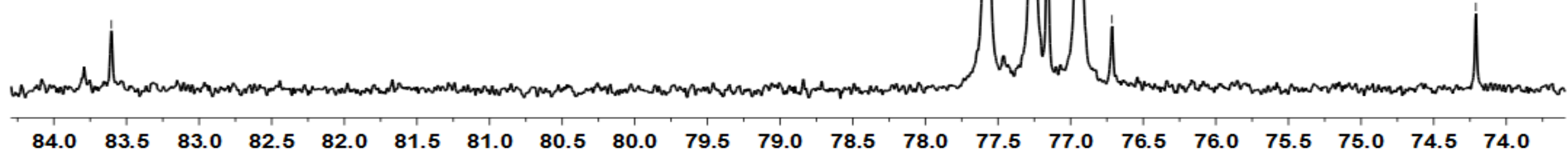




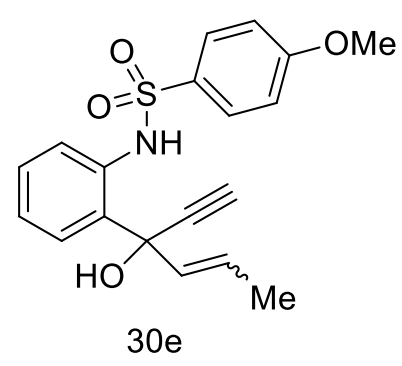

$500 \mathrm{MHz}, \mathrm{CDCl} 3$

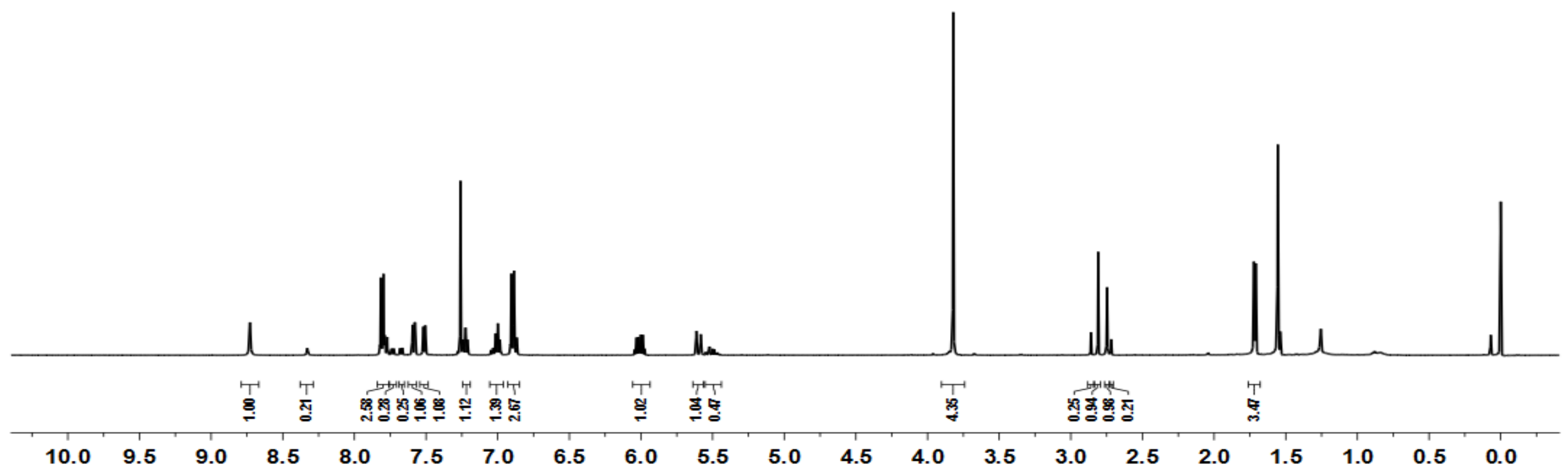




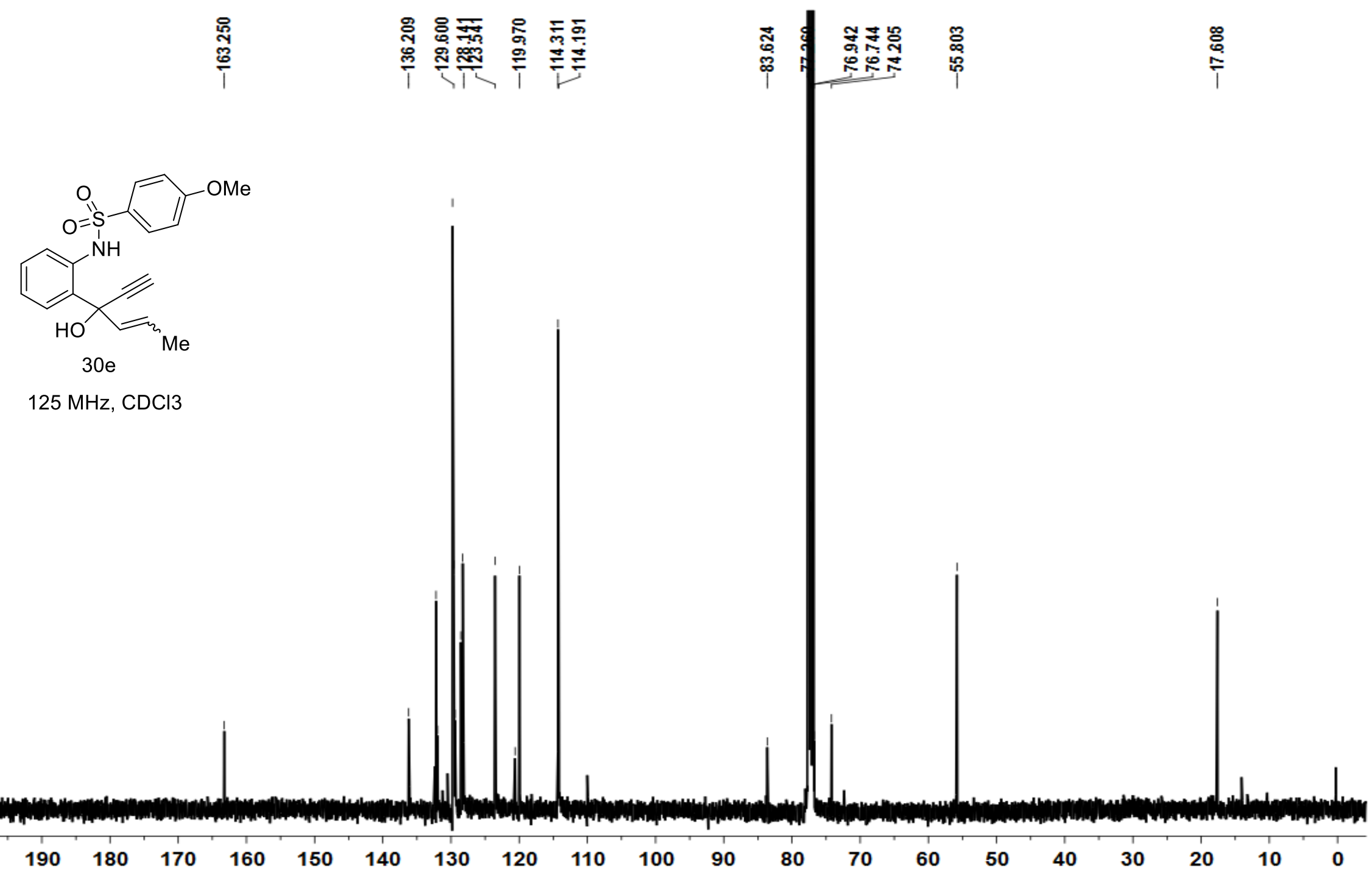




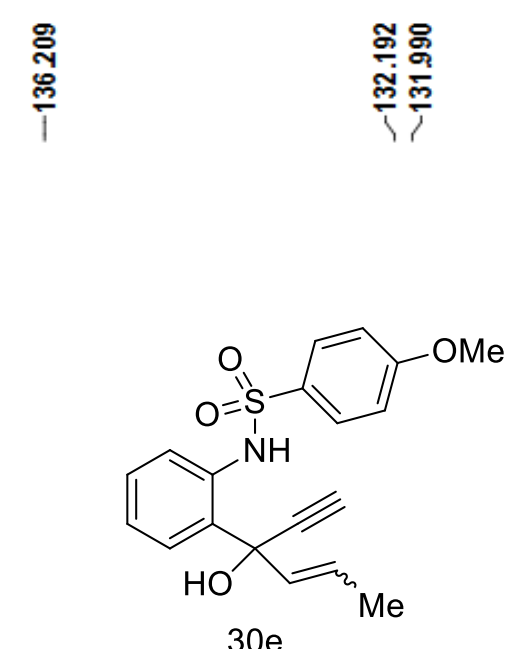

$125 \mathrm{MHz}, \mathrm{CDCl} 3$

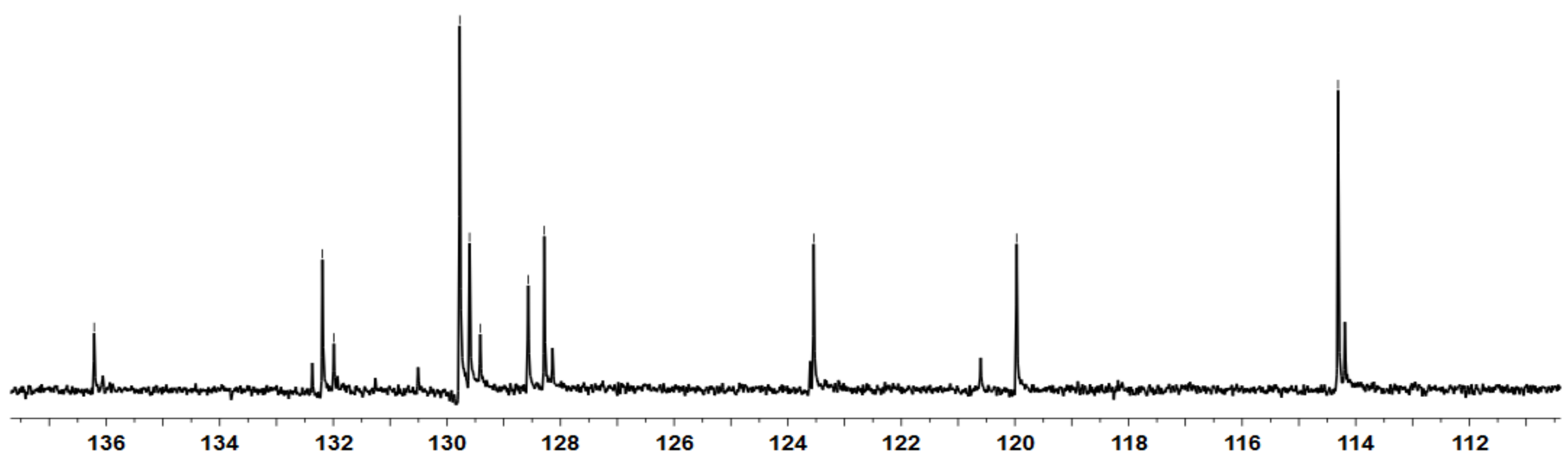




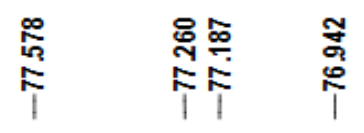

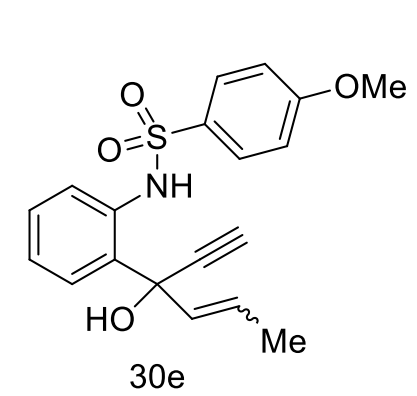

$125 \mathrm{MHz}, \mathrm{CDCl} 3$

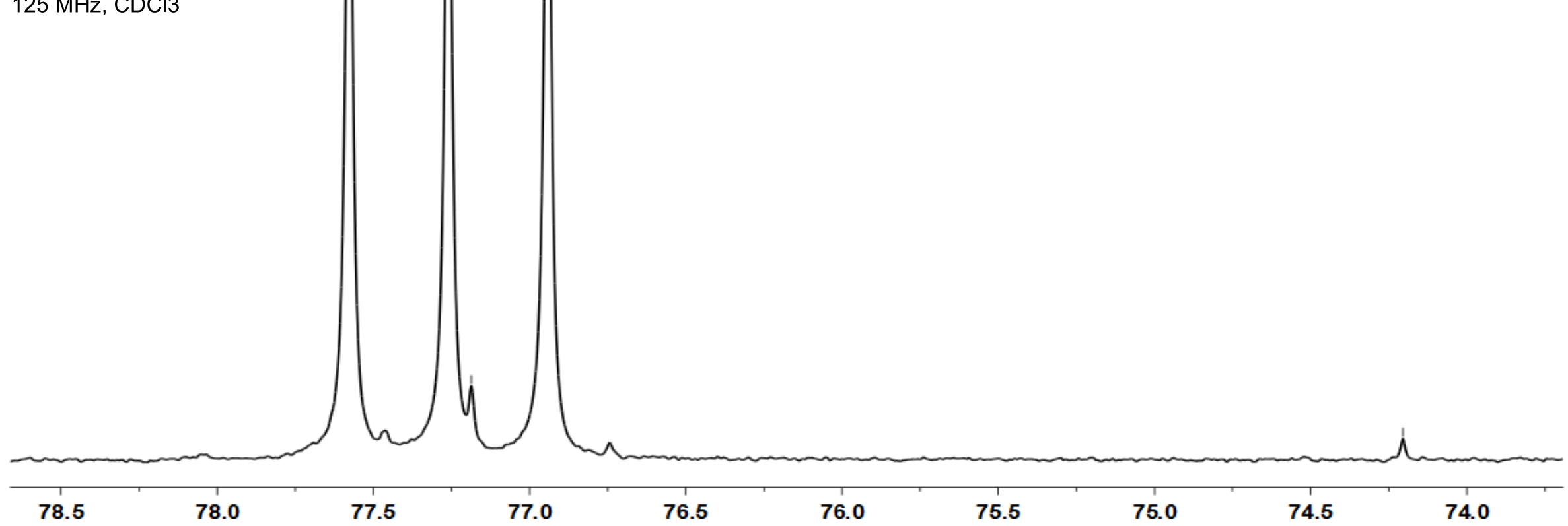




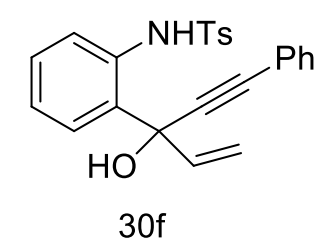

$500 \mathrm{MHz}, \mathrm{CDCl} 3$

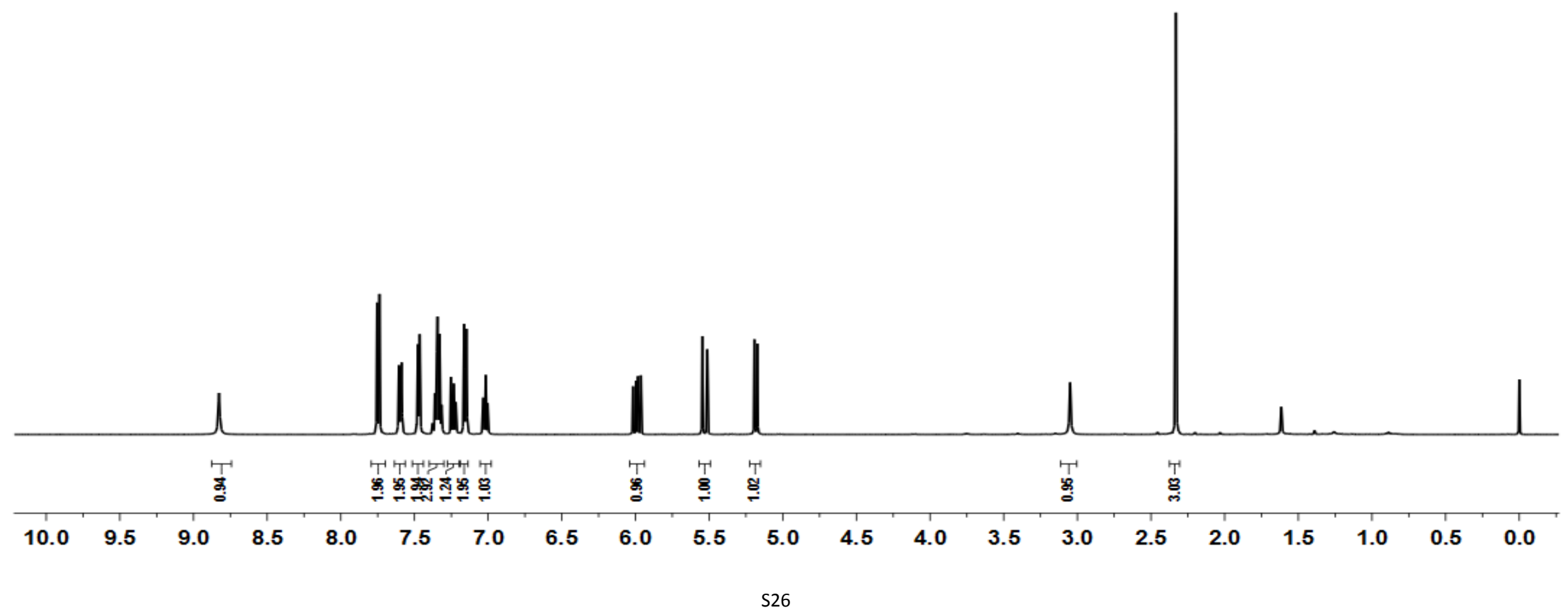




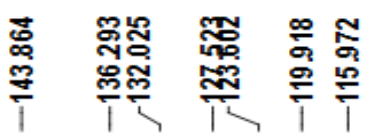

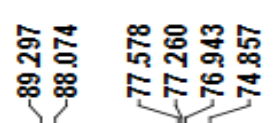

$\frac{\pi}{i}$

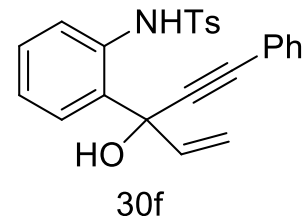

$125 \mathrm{MHz}, \mathrm{CDCl} 3$

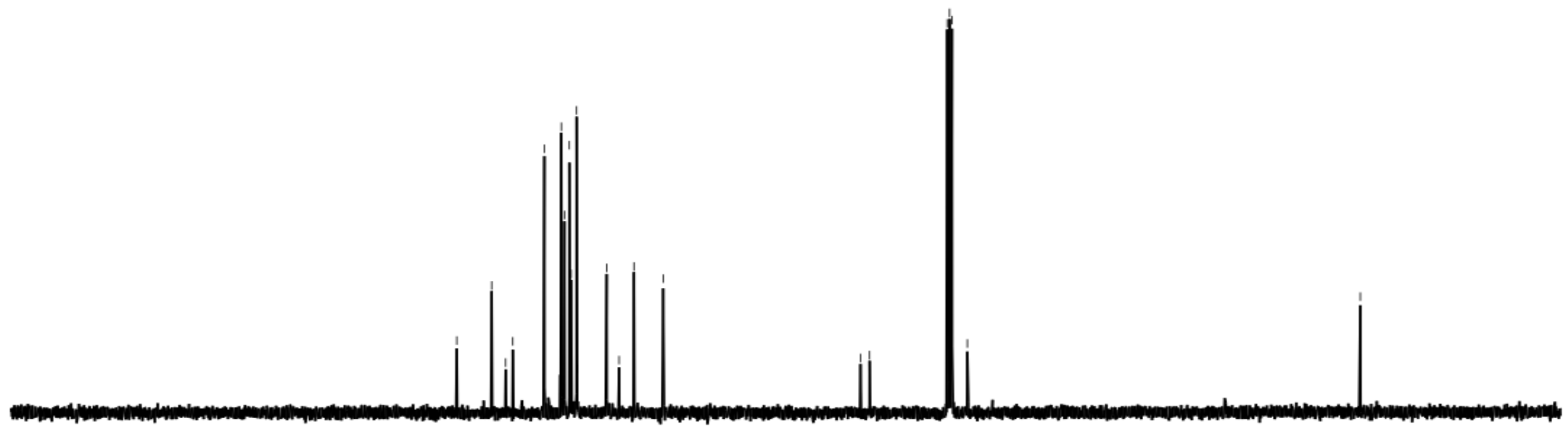




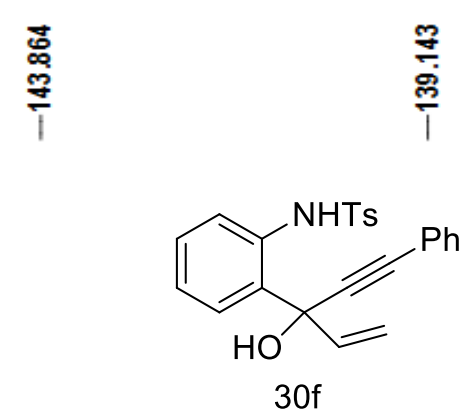

$125 \mathrm{MHz}, \mathrm{CDCl} 3$

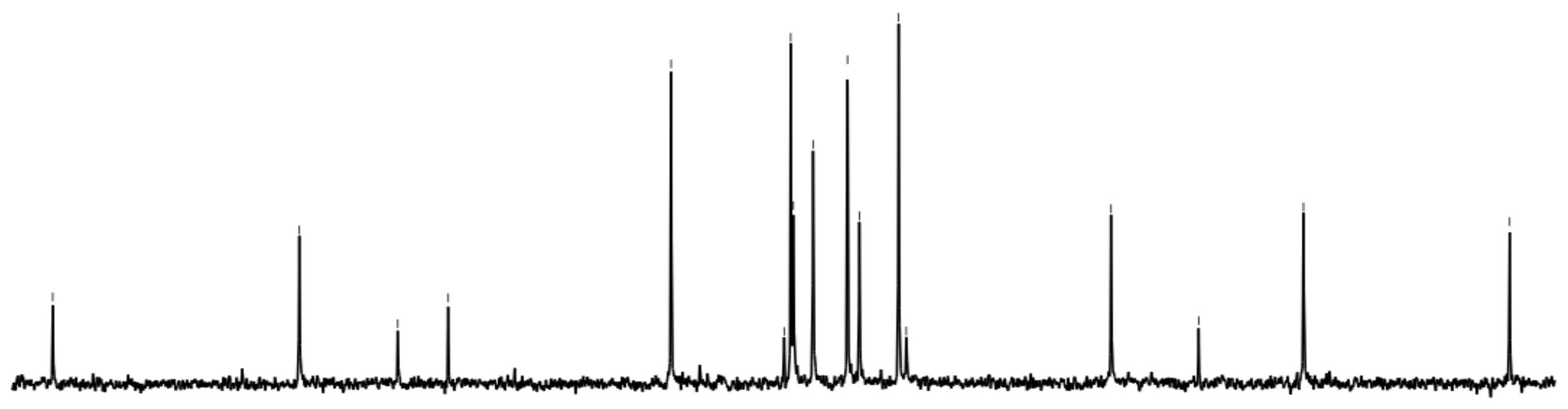




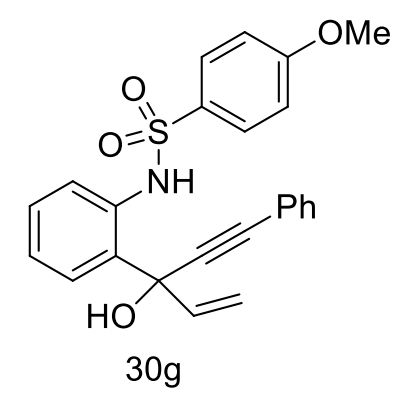

$400 \mathrm{MHz}, \mathrm{CDCl} 3$

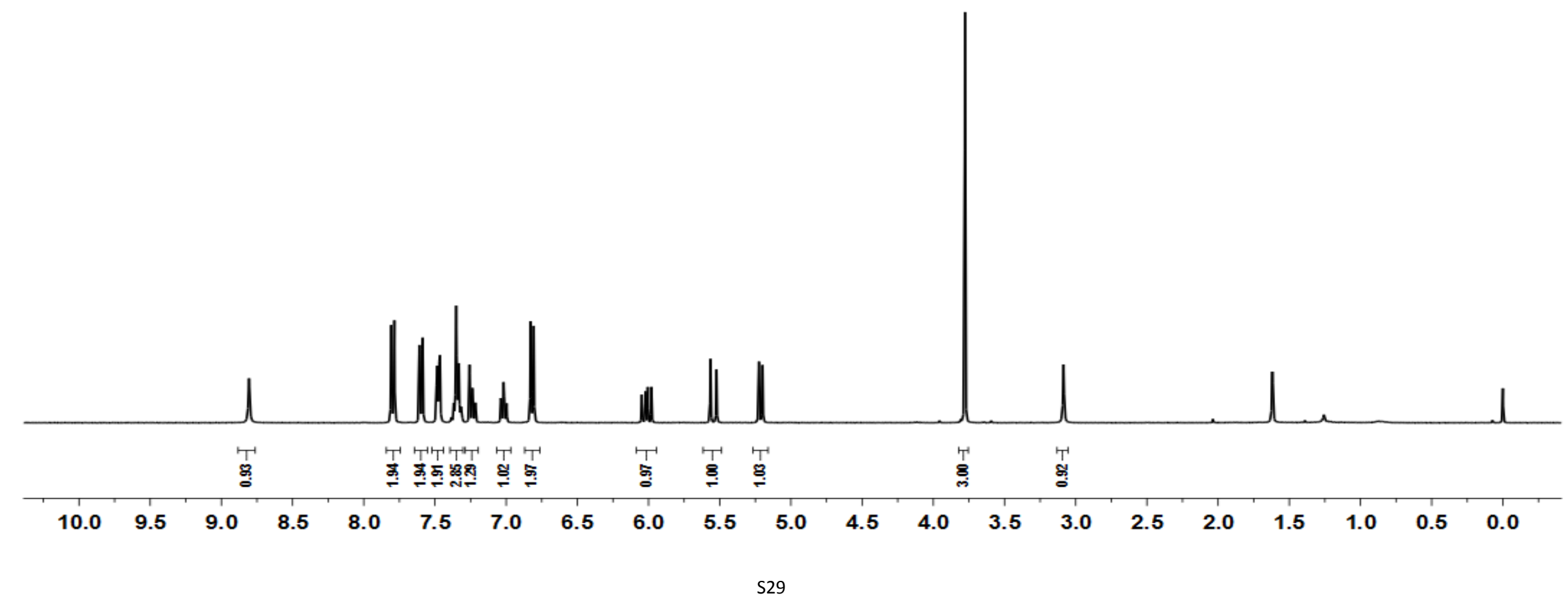




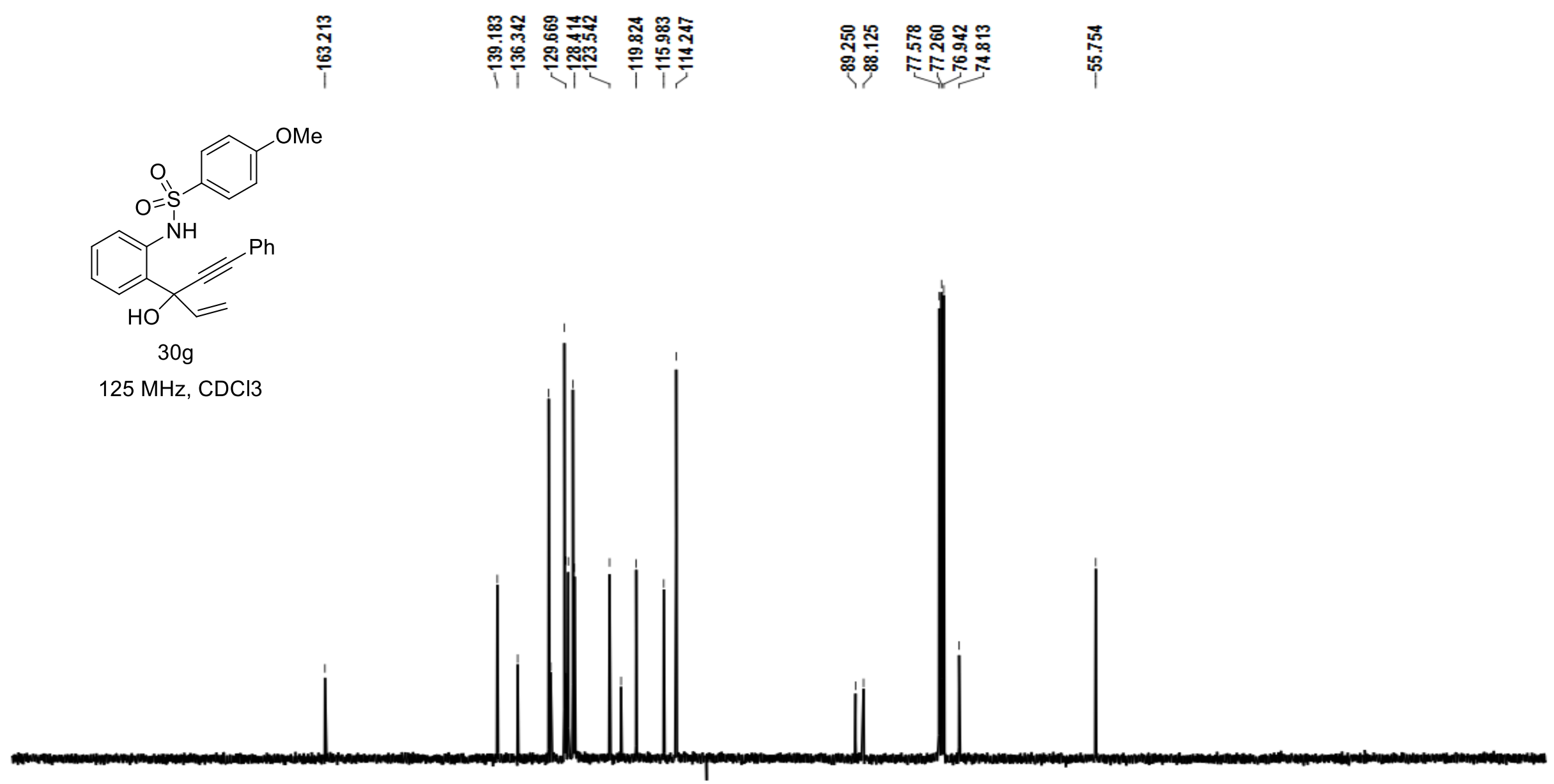




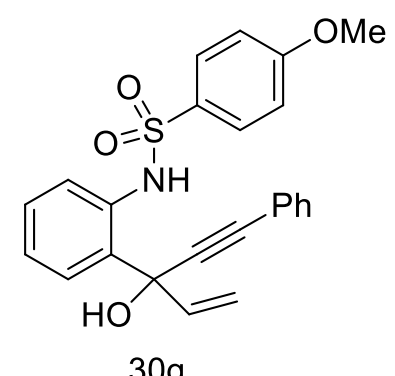

$125 \mathrm{MHz}, \mathrm{CDCl} 3$
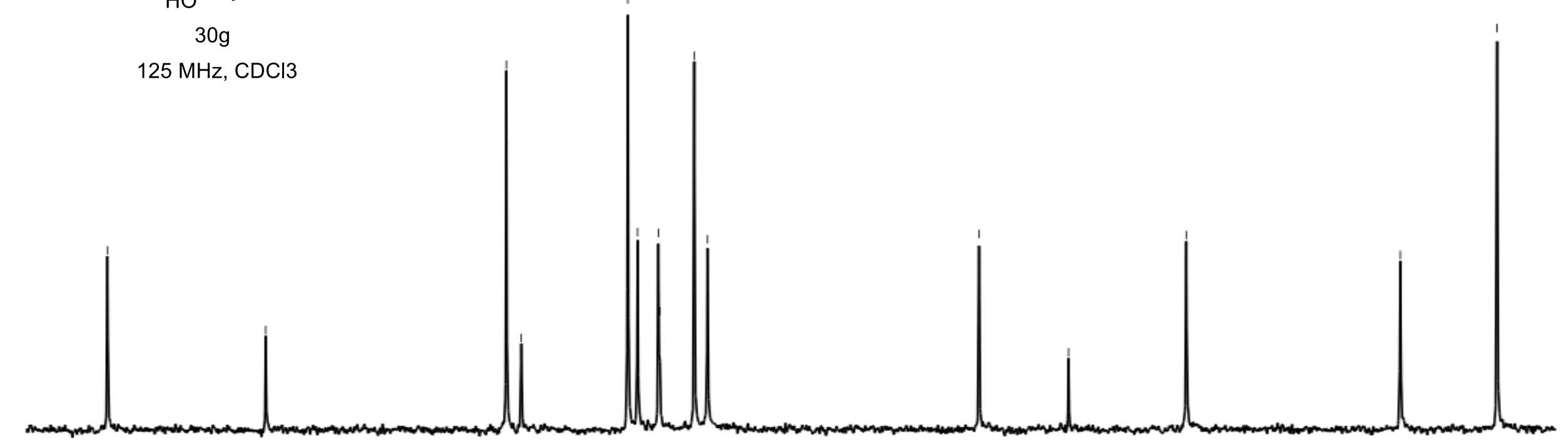

139

137

135

133

131

129

127

125

123

121

119

117 


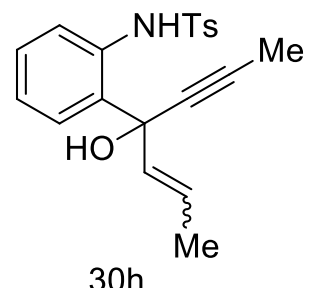

$30 \mathrm{~h}$

$400 \mathrm{MHz}, \mathrm{CDCl} 3$

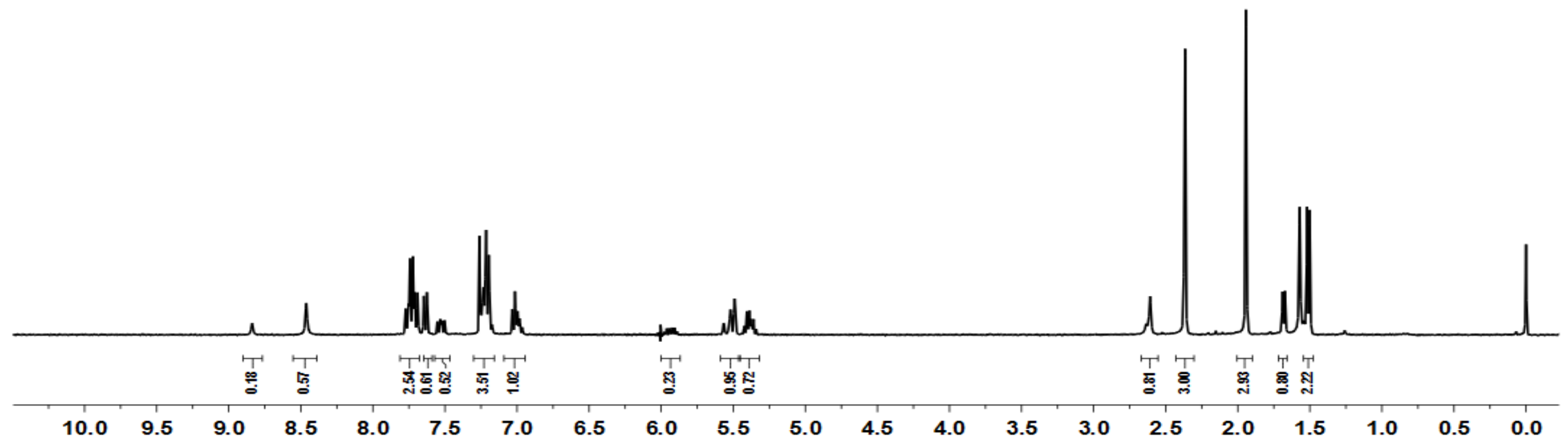




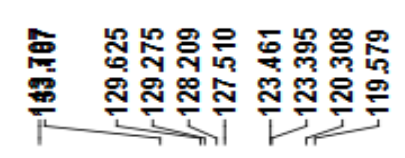

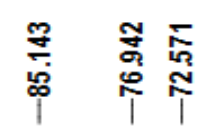

突

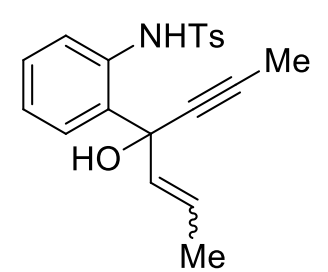

$30 \mathrm{~h}$

$125 \mathrm{MHz}, \mathrm{CDCl} 3$

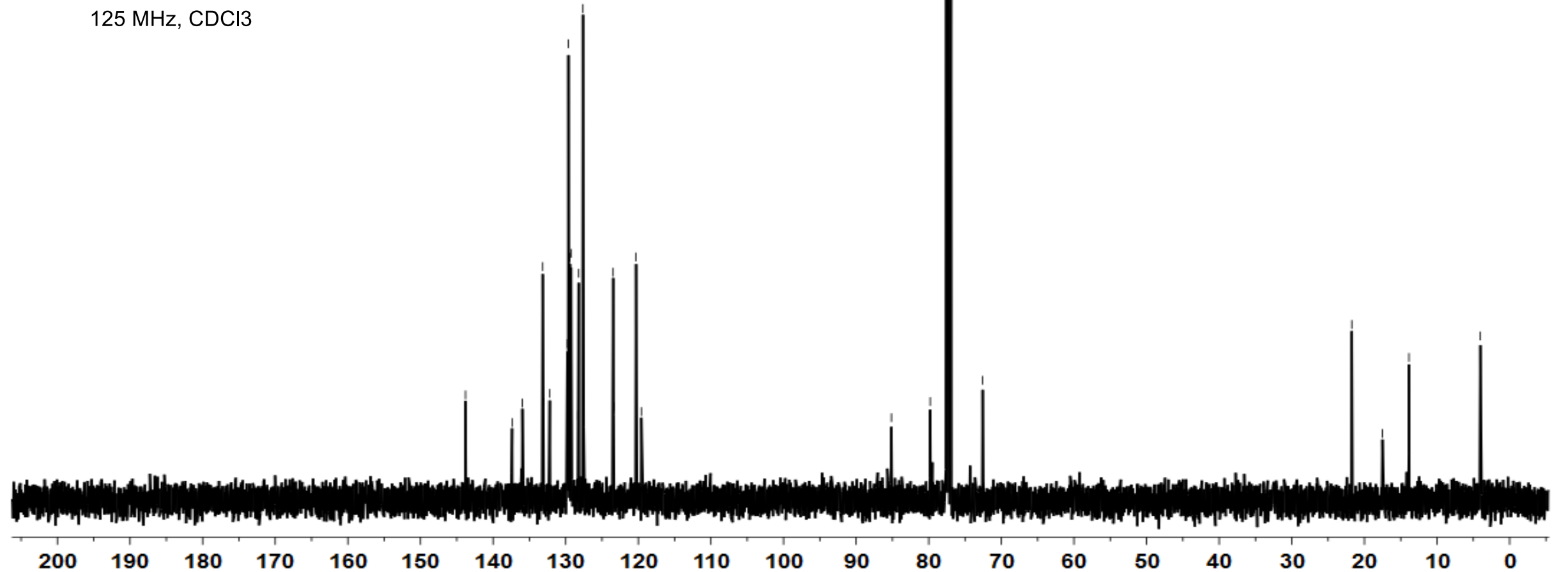




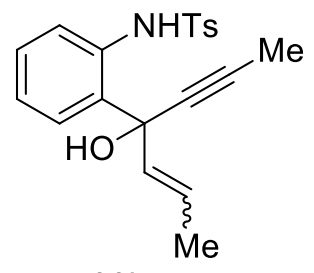

$30 \mathrm{~h}$

$125 \mathrm{MHz}, \mathrm{CDCl} 3$

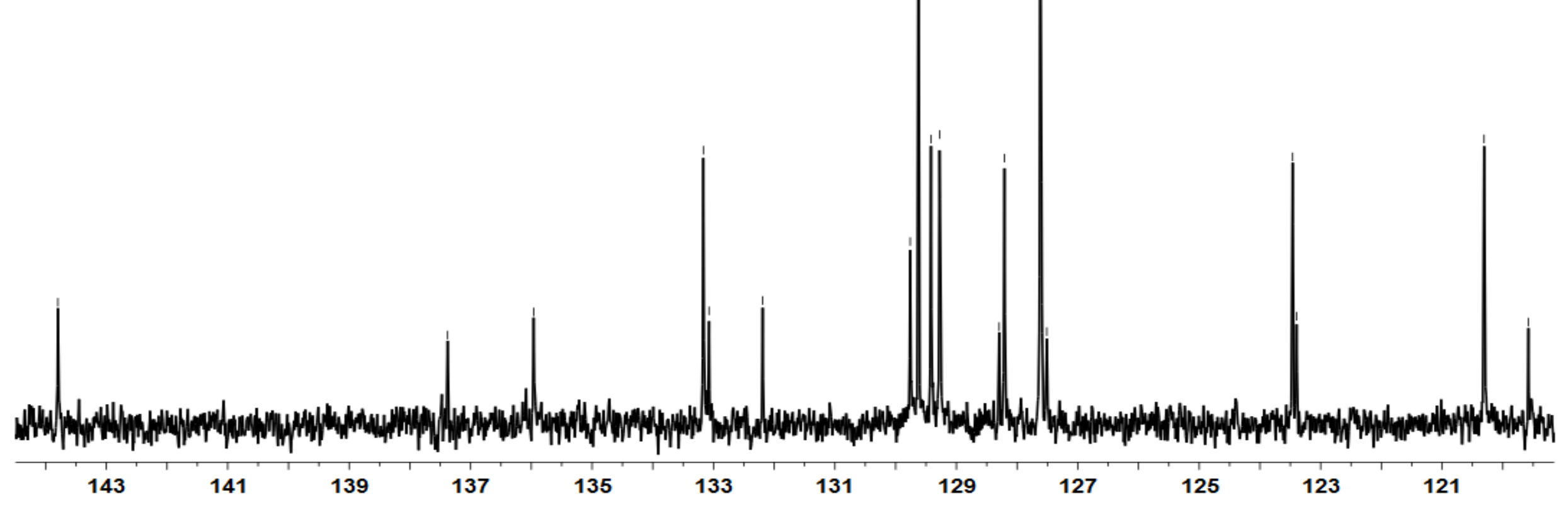




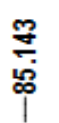

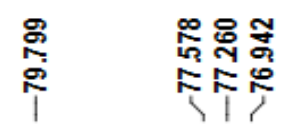

ָิธ

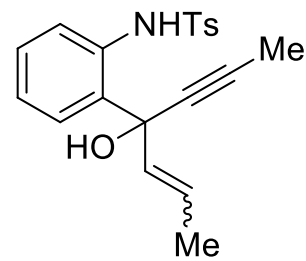

$30 \mathrm{~h}$

$125 \mathrm{MHz}, \mathrm{CDCl} 3$

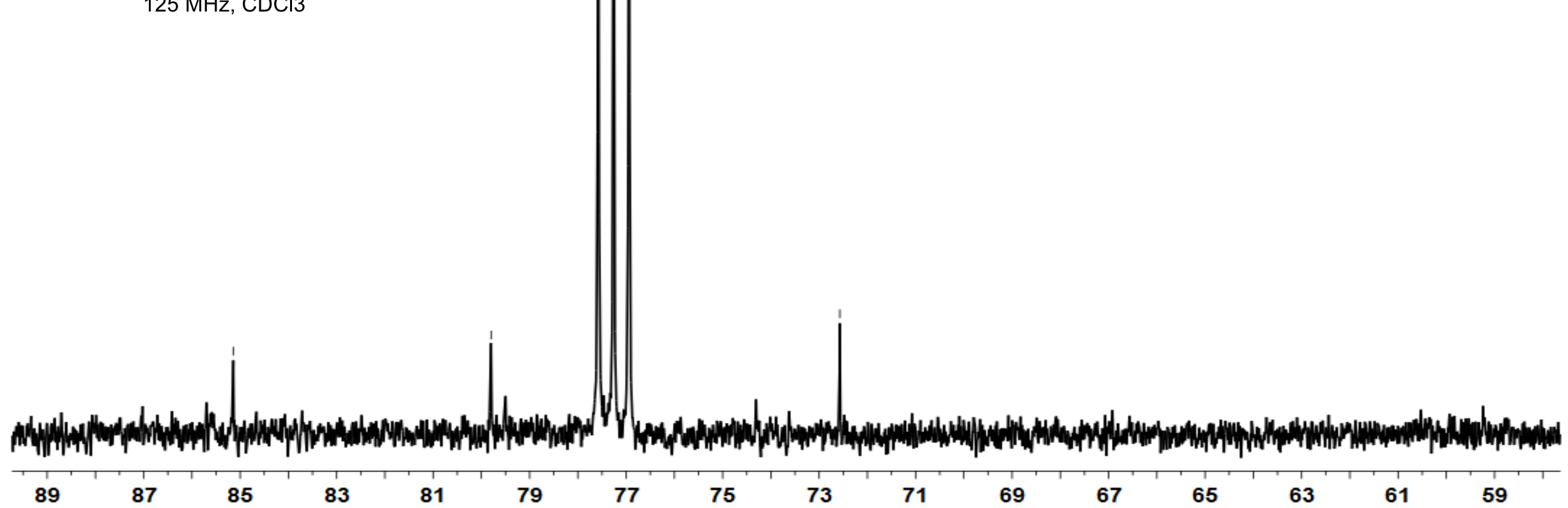




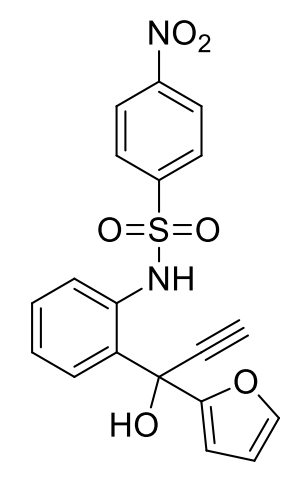

$36 b$

$400 \mathrm{MHz}, \mathrm{CDCl} 3$

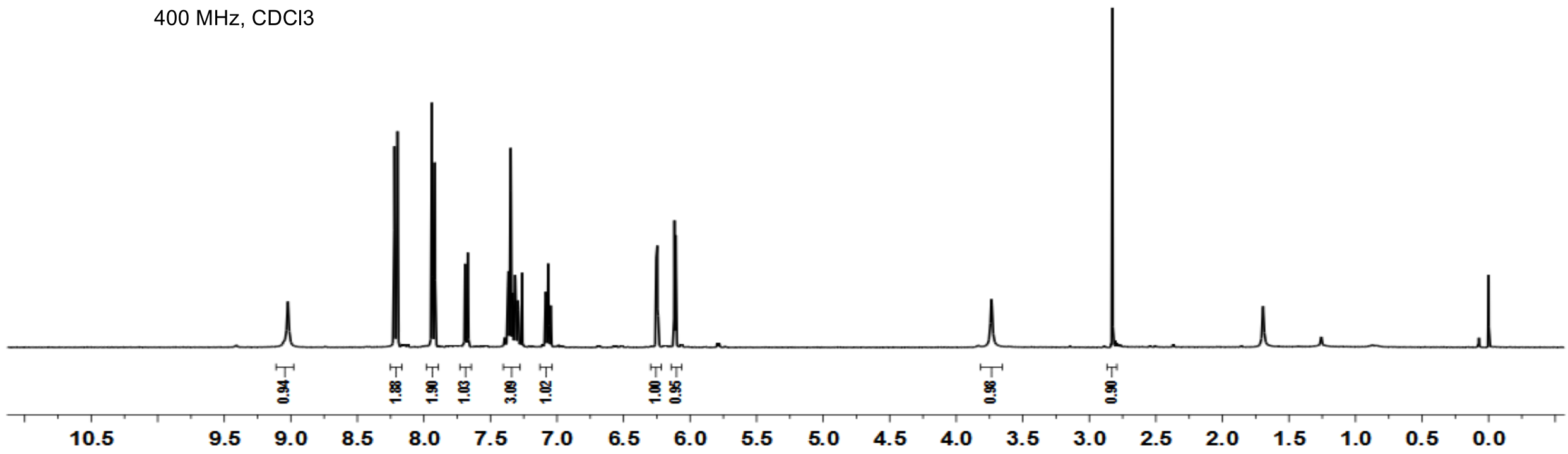




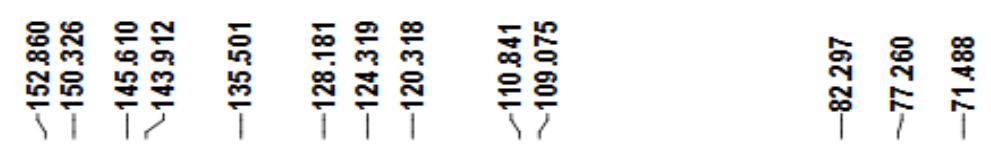

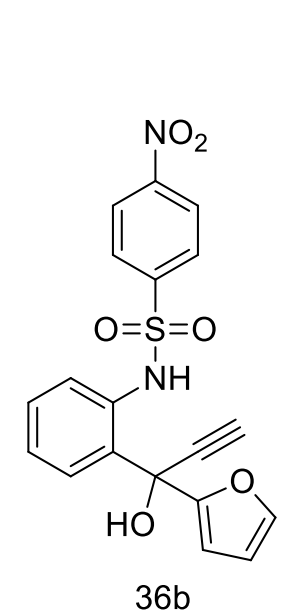

$100 \mathrm{MHz}, \mathrm{CDCl} 3$

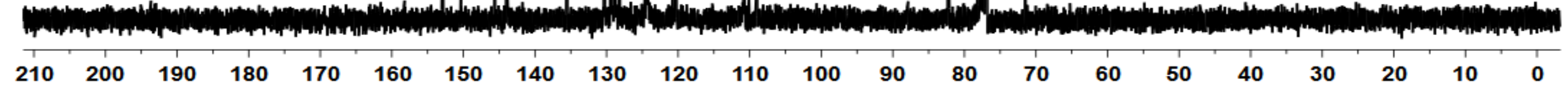




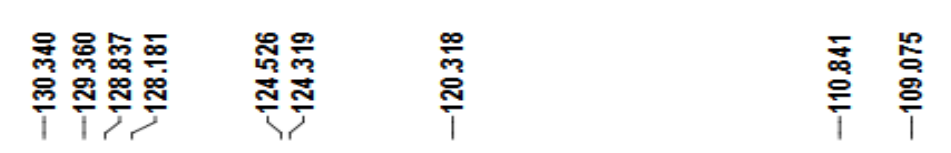

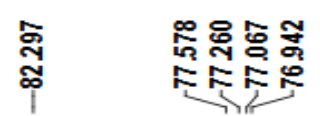

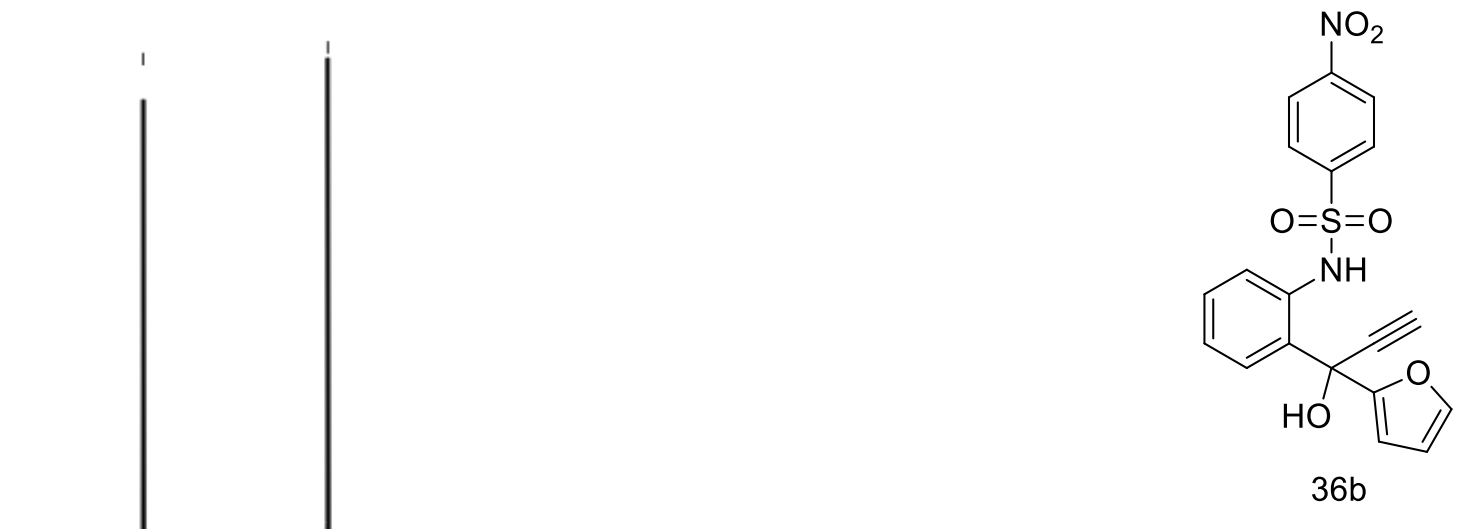

$100 \mathrm{MHz}, \mathrm{CDCl} 3$

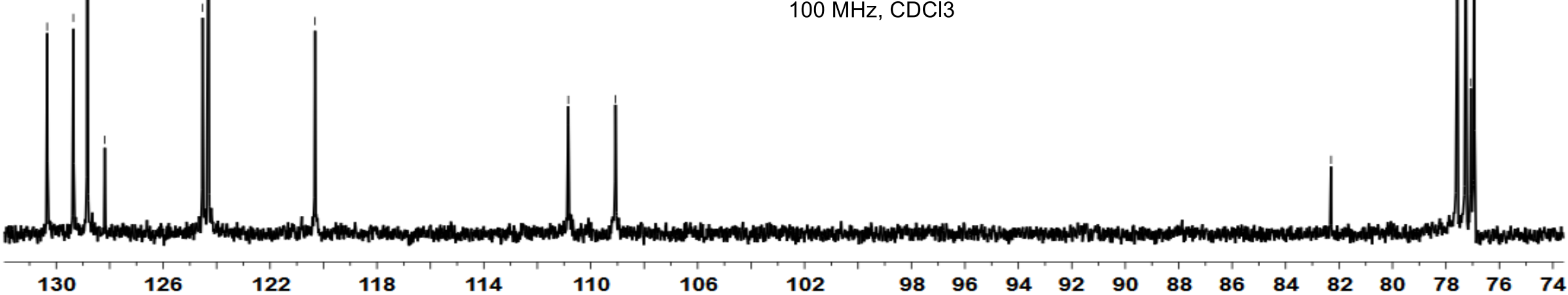




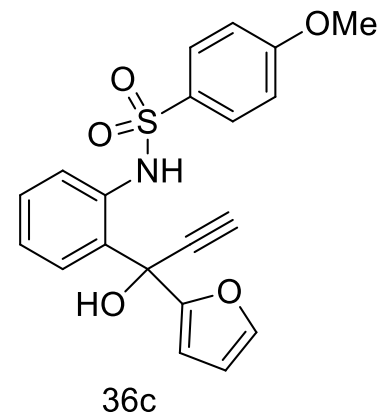

$400 \mathrm{MHz}, \mathrm{CDCl} 3$

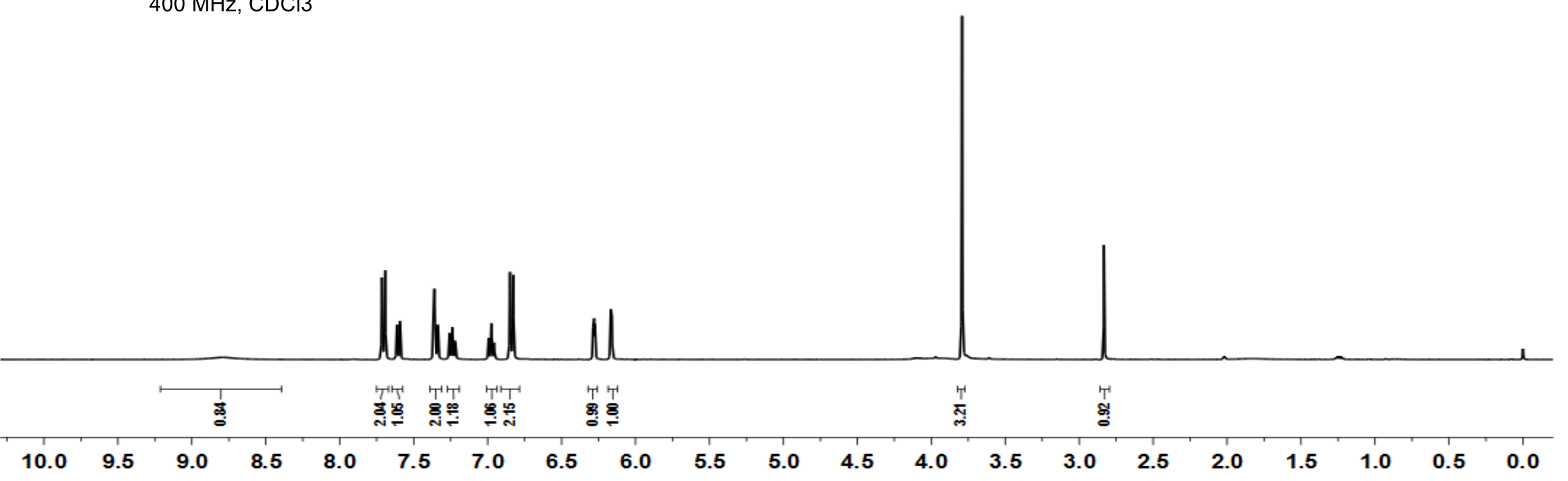




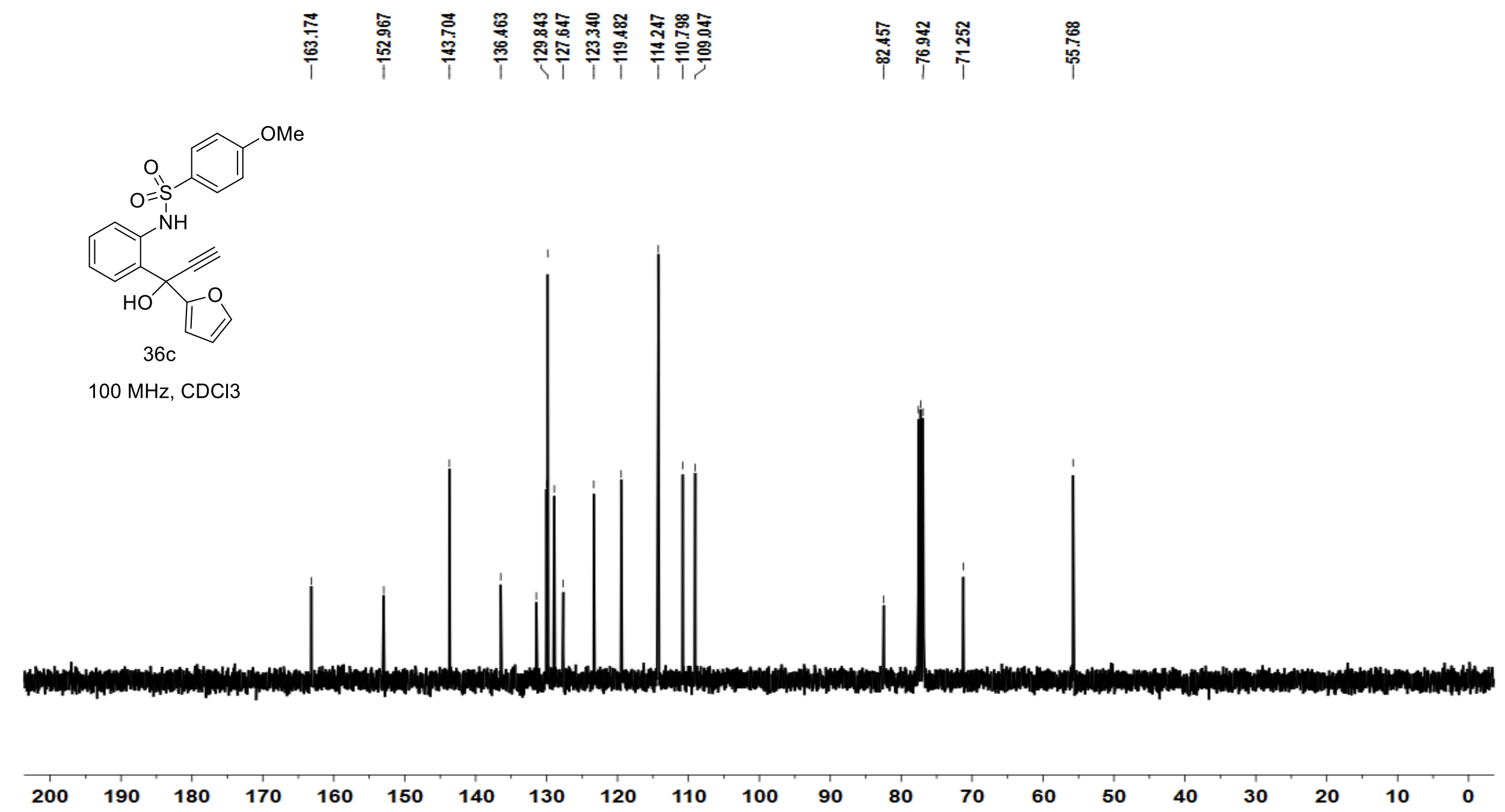



$\frac{\mathscr{8}}{\mathbf{8}}$

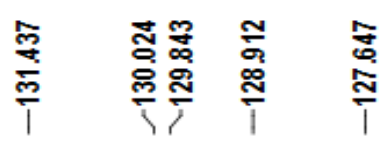
i̊

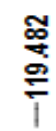
$\stackrel{\text { F }}{\stackrel{7}{7}}$

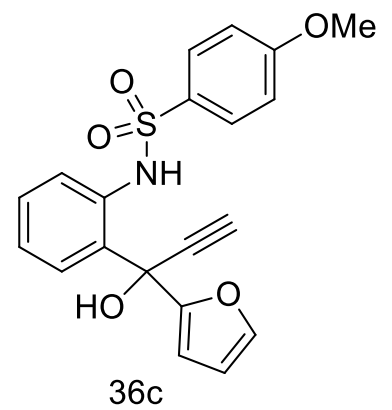

$100 \mathrm{MHz}, \mathrm{CDCl} 3$

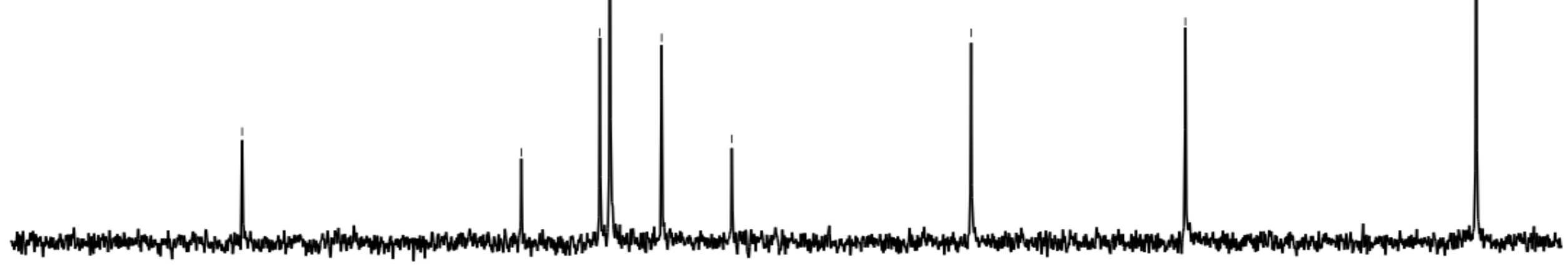




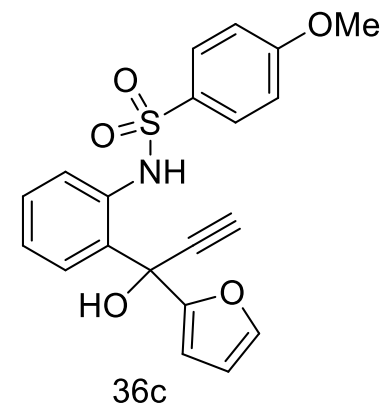

$100 \mathrm{MHz}, \mathrm{CDCl} 3$

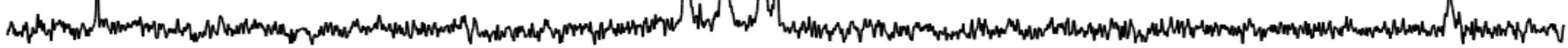

\begin{tabular}{|c|c|c|c|c|c|}
\hline 83.0 & 82.0 & 81.0 & 80.0 & 79.0 & 78.0 \\
\hline
\end{tabular}




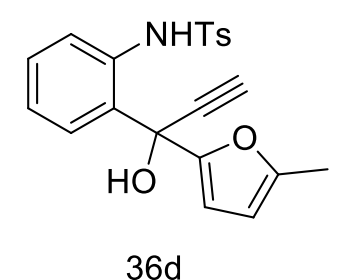

$500 \mathrm{MHz}, \mathrm{CDCl} 3$

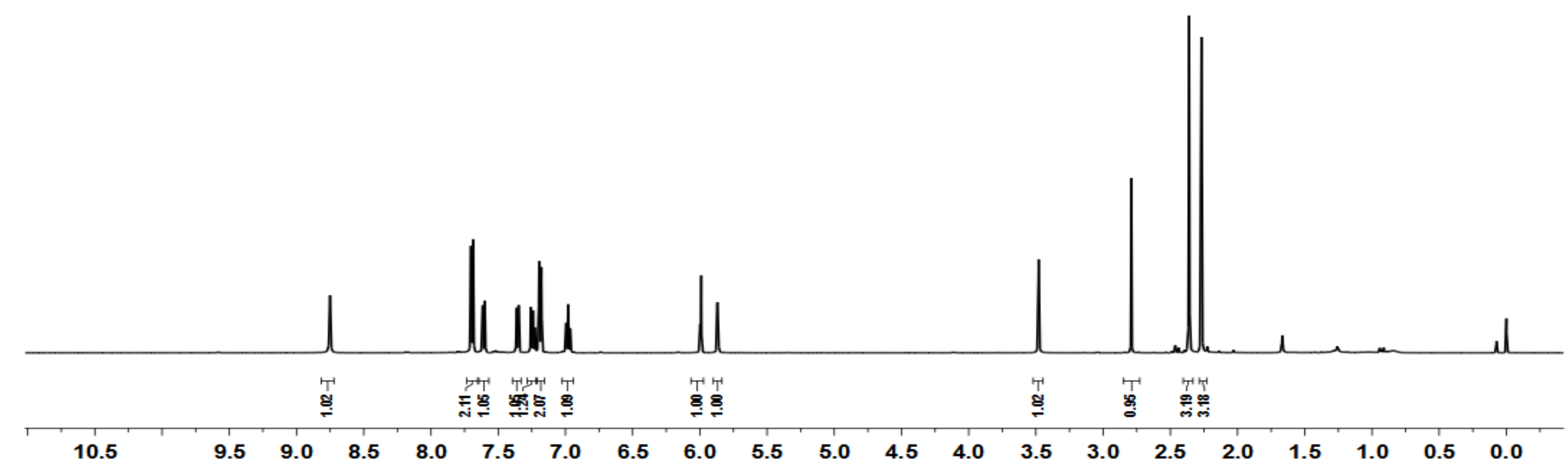




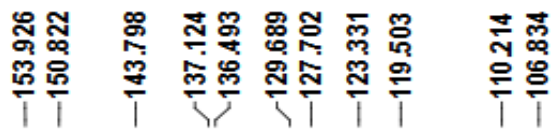

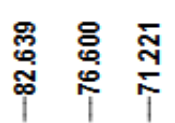

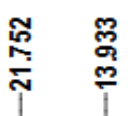

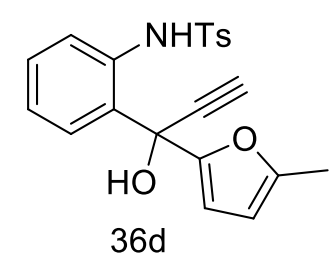

$100 \mathrm{MHz}, \mathrm{CDCl} 3$

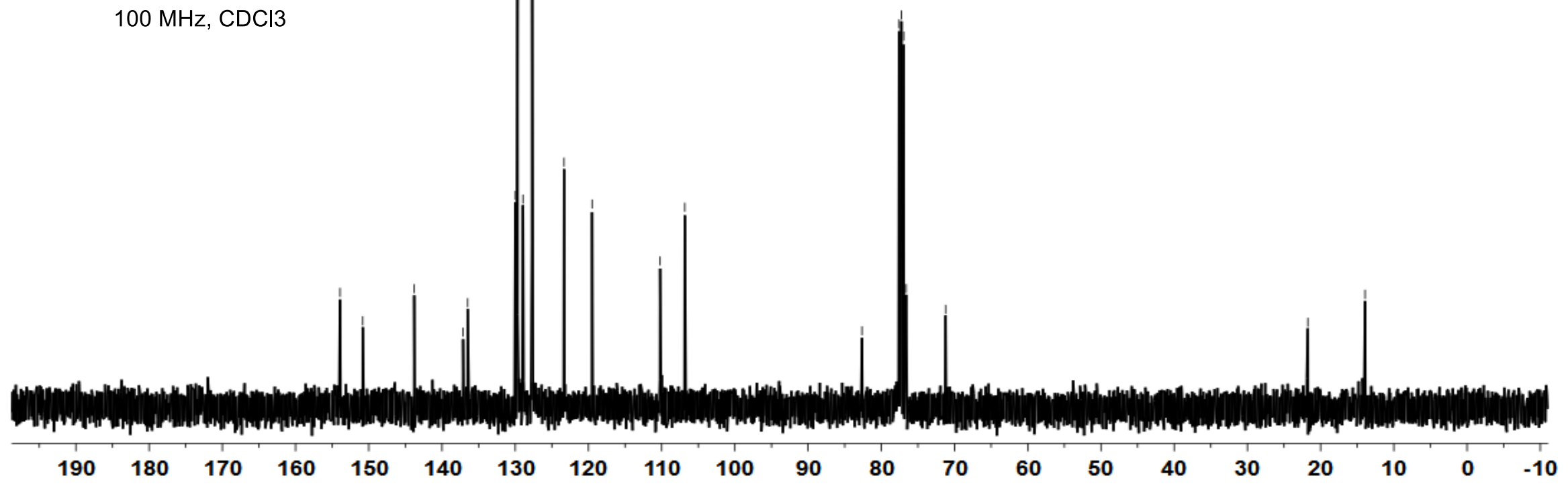




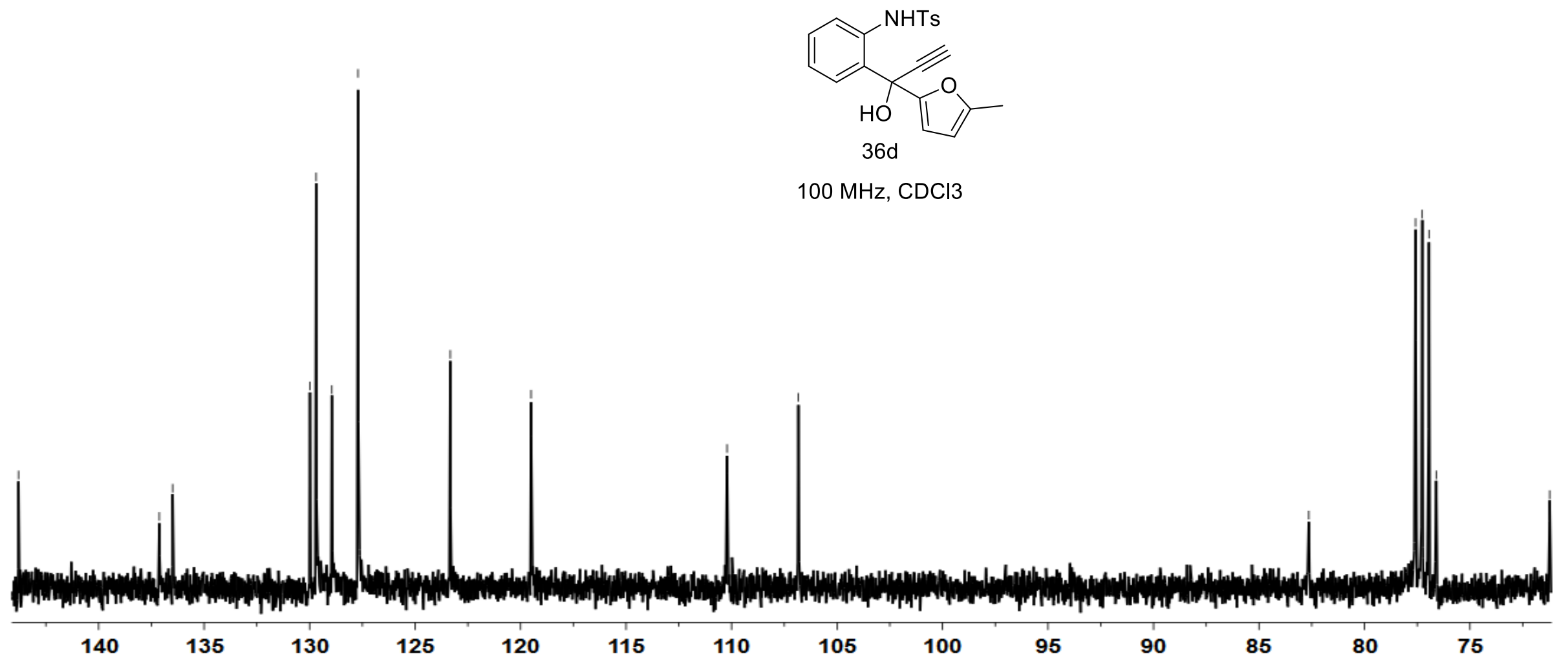




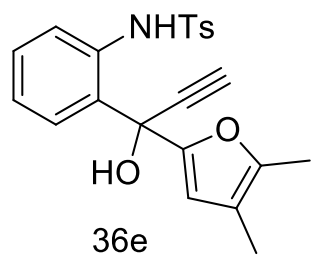

$500 \mathrm{MHz}, \mathrm{CDCl} 3$

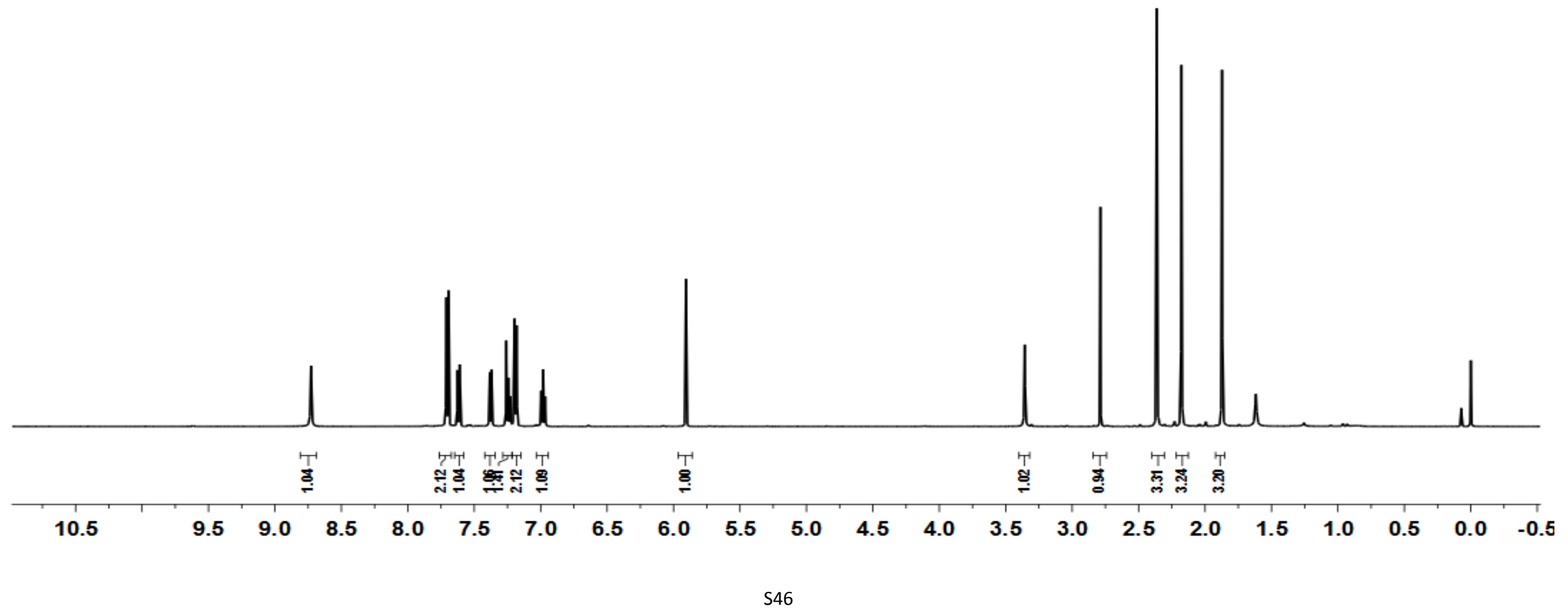




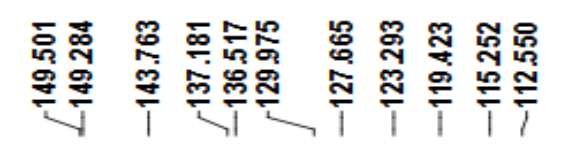

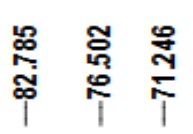

꿍 흉유

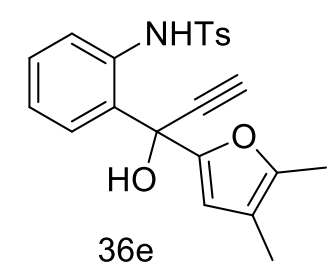

$125 \mathrm{MHz}, \mathrm{CDCl} 3$
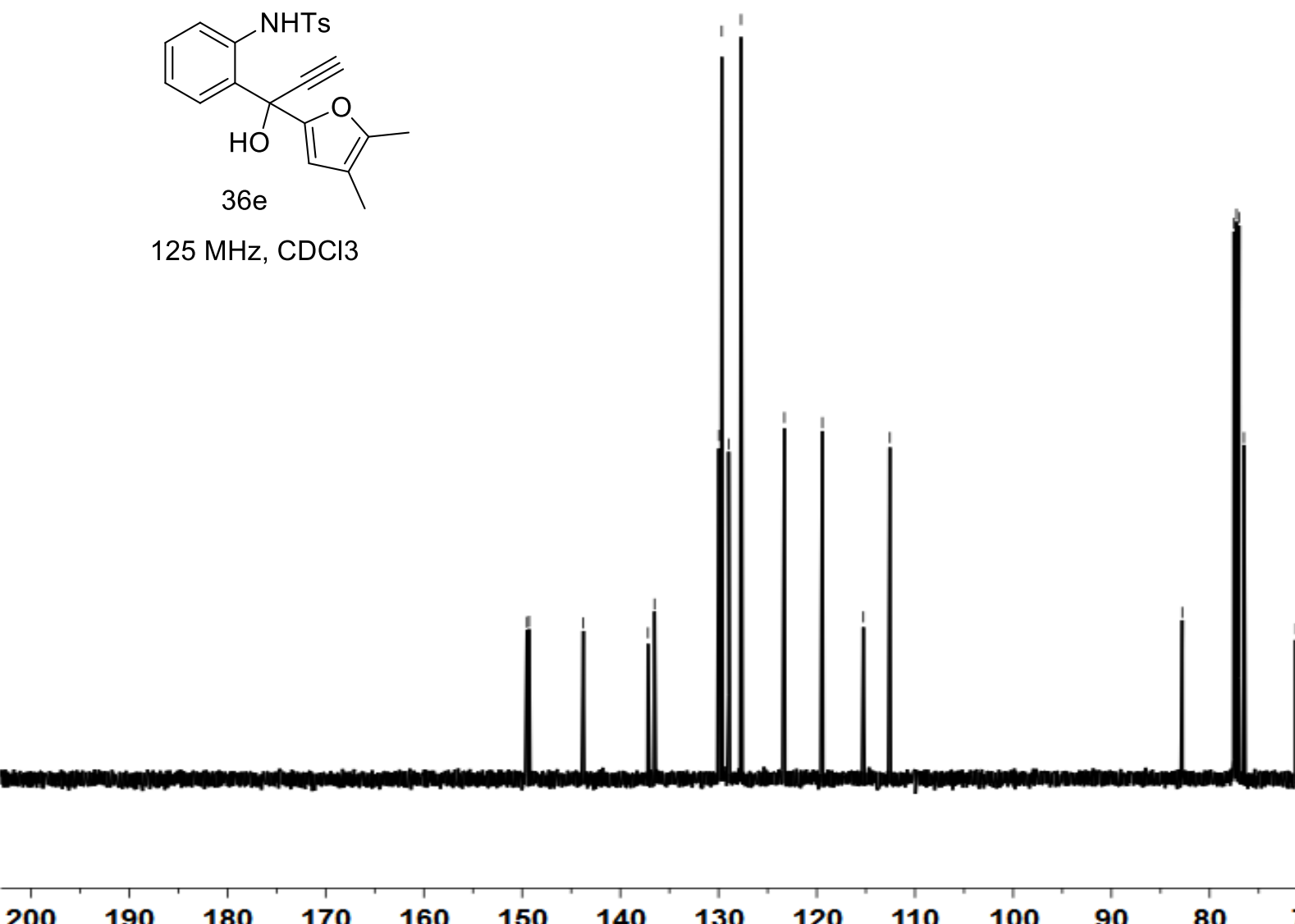

150

140

130

120

110

100

90

80

7060

50

40

30

20

100 


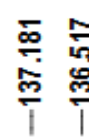

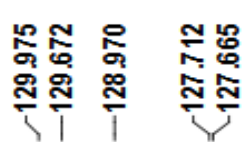

ঙ્స్త్

$\stackrel{\dddot{7}}{\frac{\pi}{7}}$

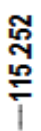

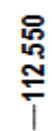

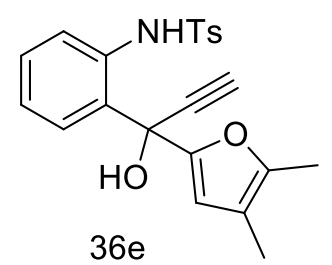

$125 \mathrm{MHz}, \mathrm{CDCl} 3$ 


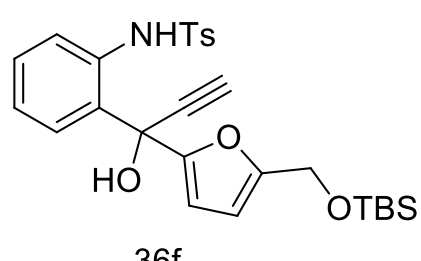

36

$400 \mathrm{MHz}, \mathrm{CDCl} 3$

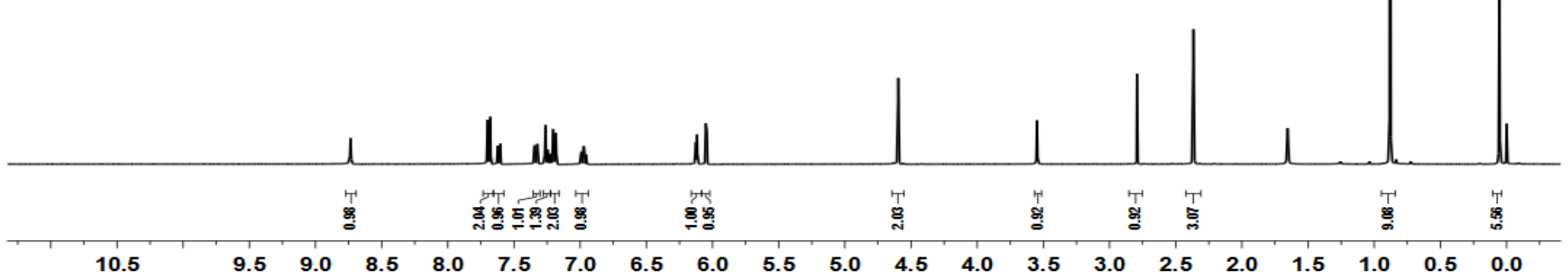




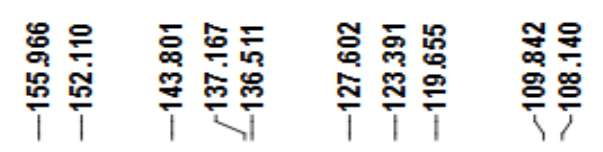

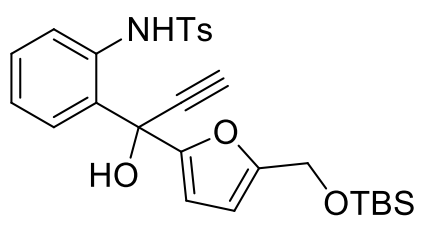

$36 f$

$100 \mathrm{MHz}, \mathrm{CDCl} 3$

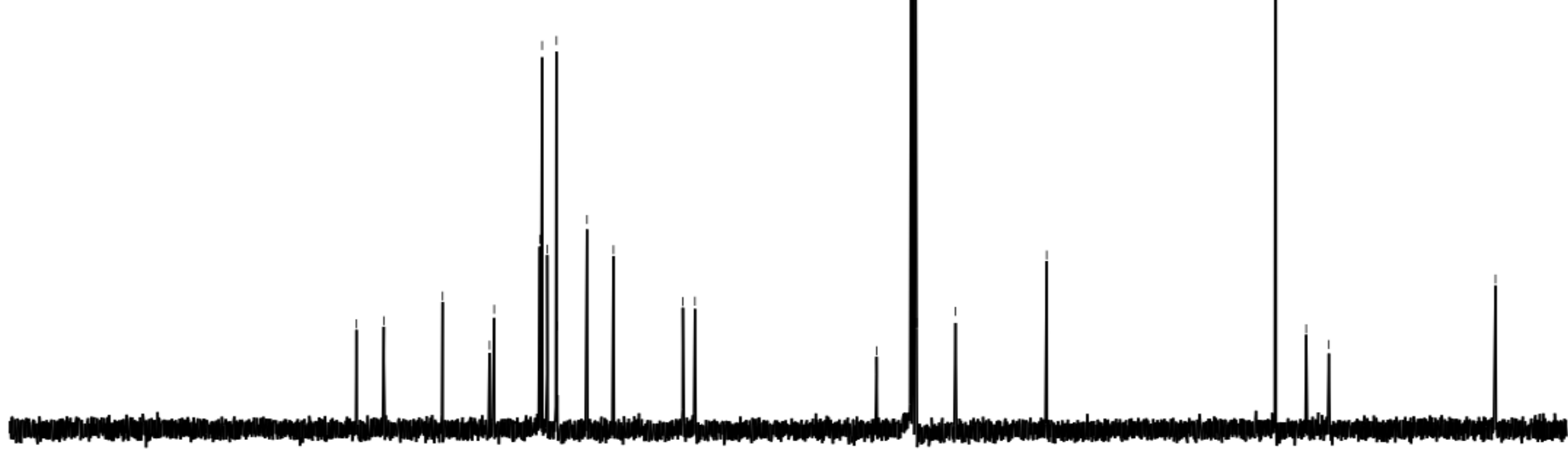

$\begin{array}{lllllllllll}200 & 190 & 180 & 170 & 160 & 150 & 140 & 130 & 120 & 110 & 100\end{array}$ 
$\begin{array}{ll}\frac{5}{1} & = \\ \frac{1}{1} & 0\end{array}$

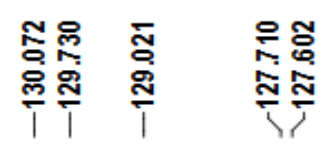

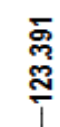

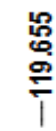

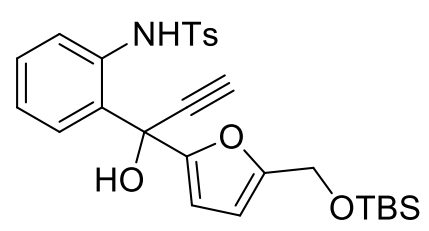

$36 f$

$100 \mathrm{MHz}, \mathrm{CDCl} 3$

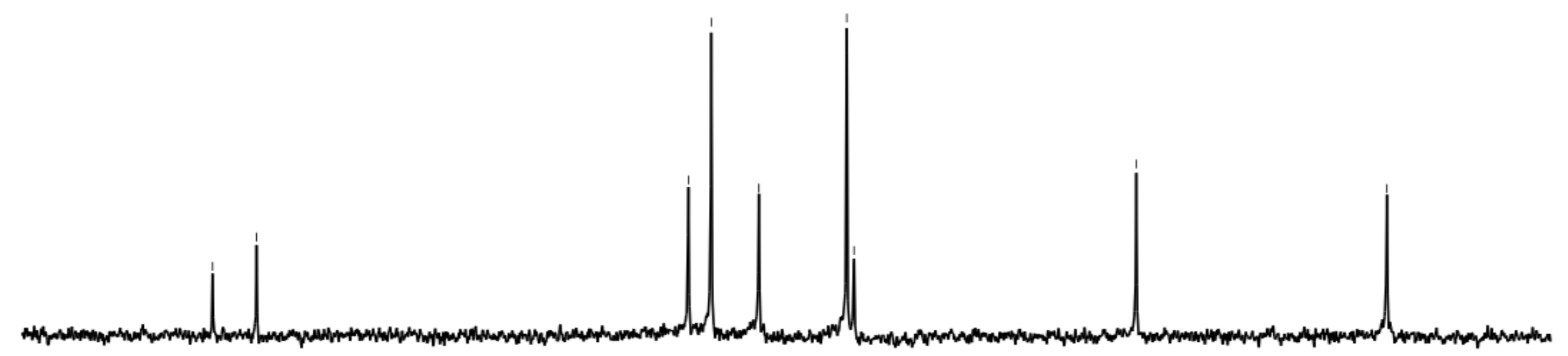

$\begin{array}{llllllllllll}140 & 139 & 138 & 137 & 136 & 135 & 134 & 133 & 132 & 131 & 130 & 129\end{array}$ 


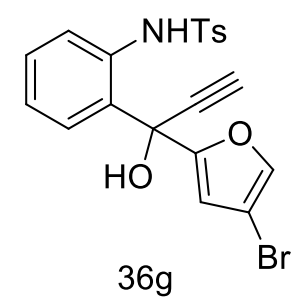

$500 \mathrm{MHz}, \mathrm{CDCl} 3$

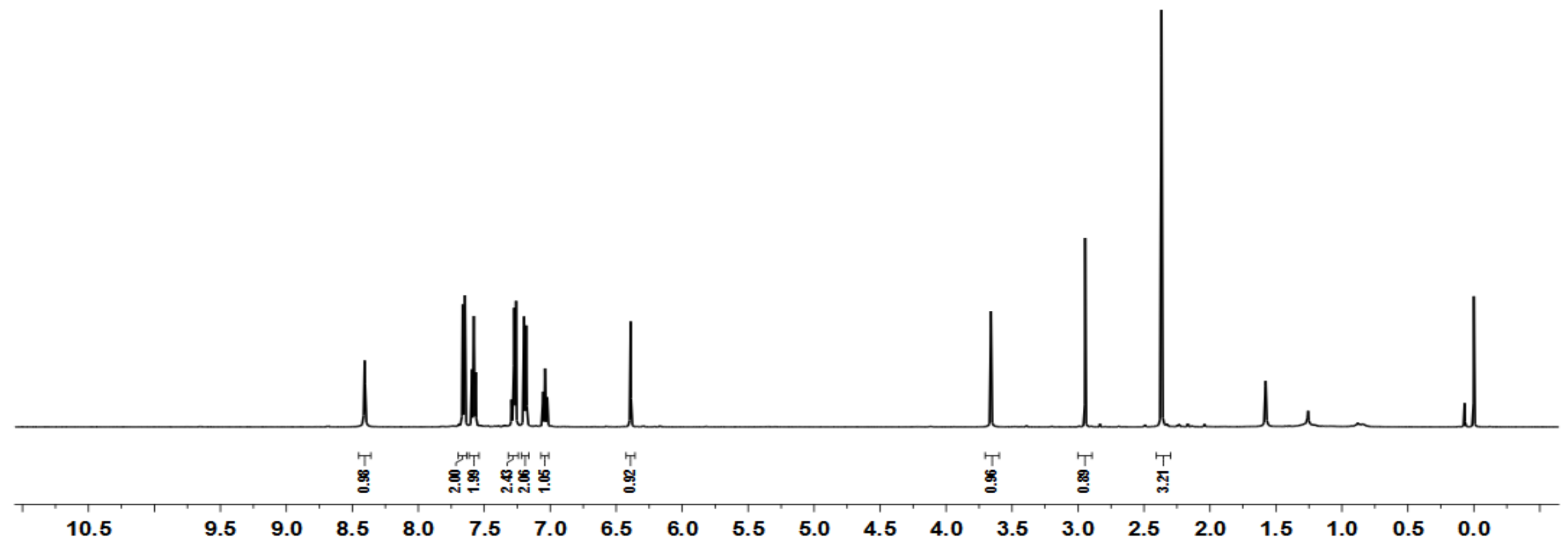




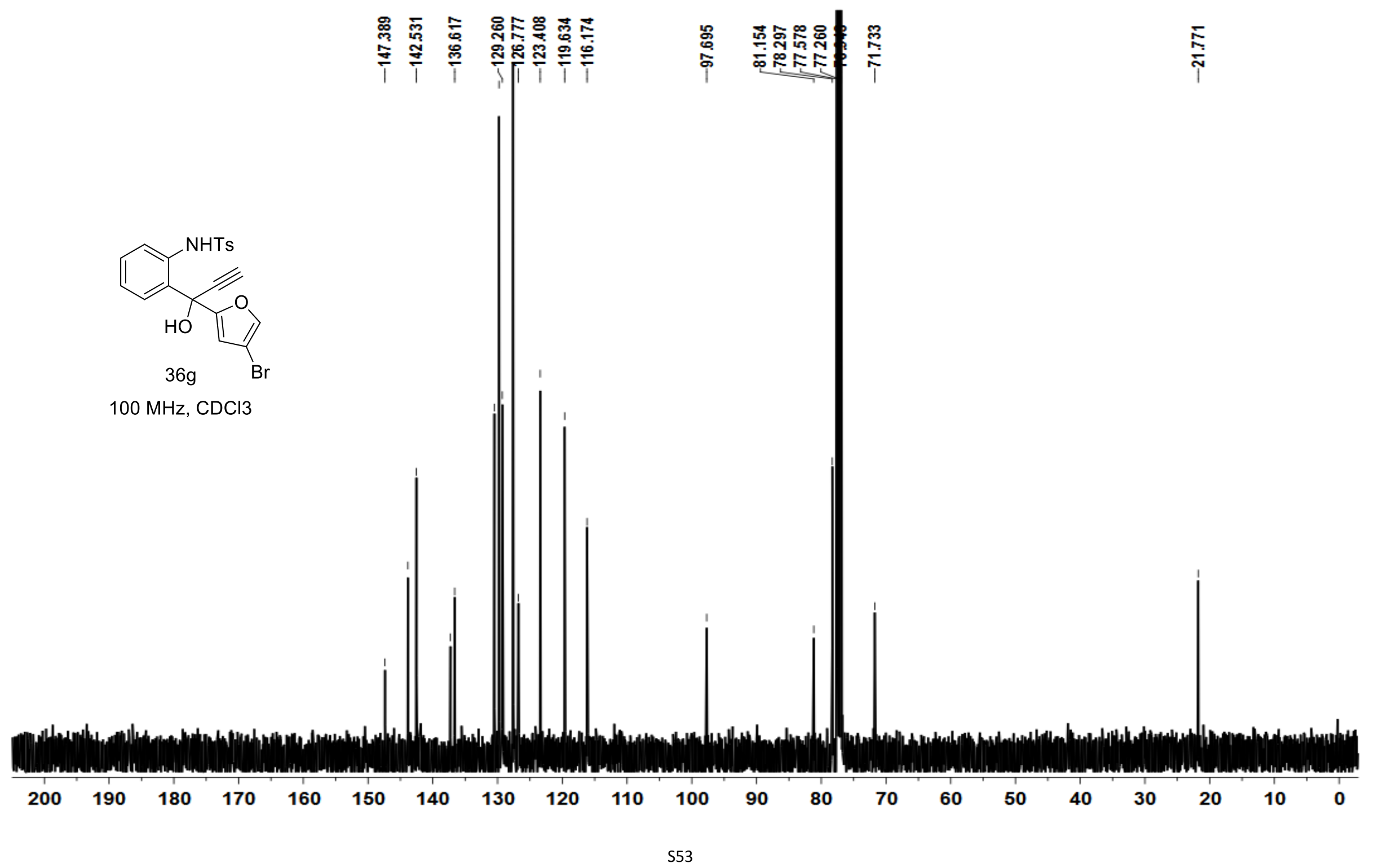




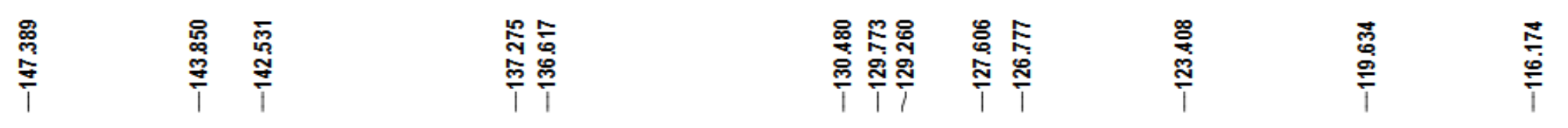

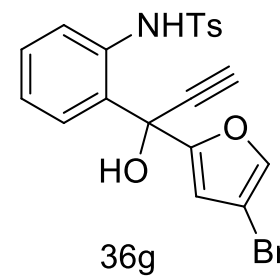

$100 \mathrm{MHz}, \mathrm{CDCl} 3$

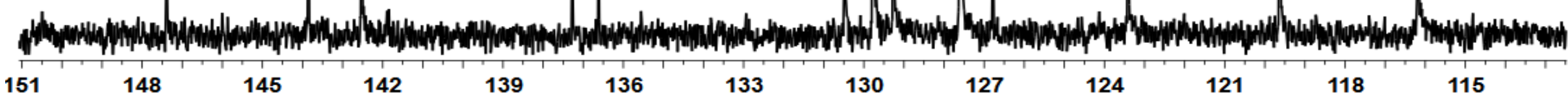




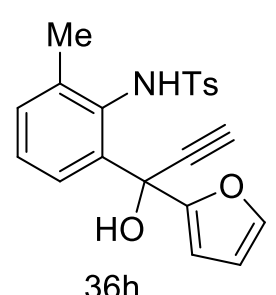

$400 \mathrm{MHz}, \mathrm{CDCl} 3$

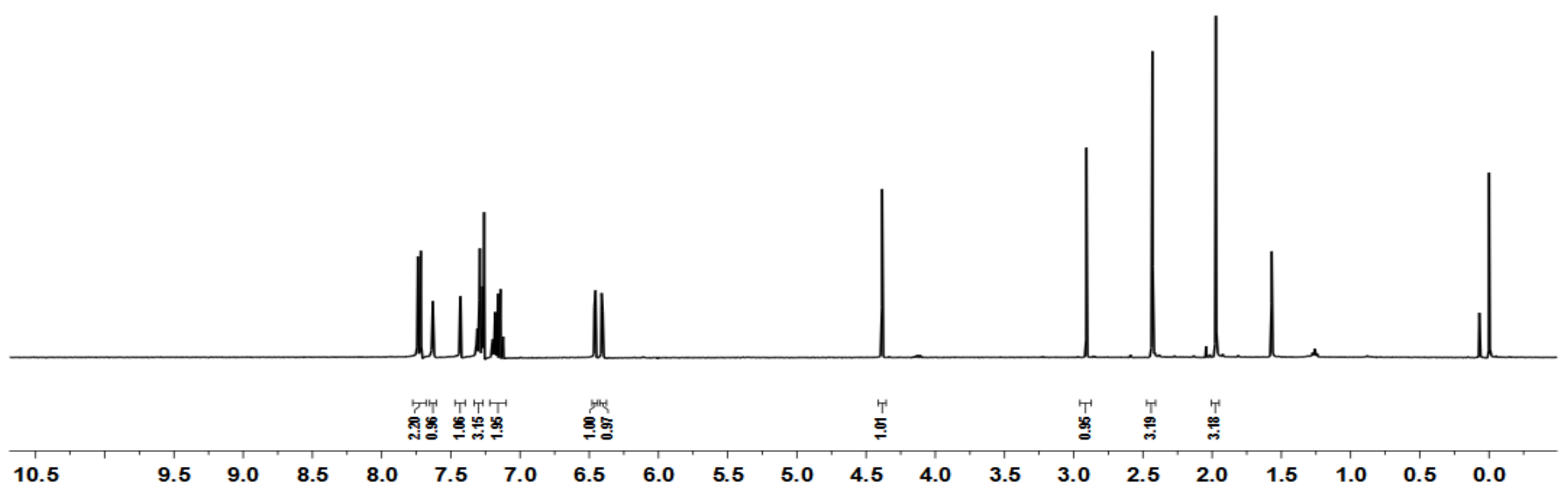




$$
\frac{\text { 急 }}{\frac{8}{5}}
$$$$
\text { 空 }
$$

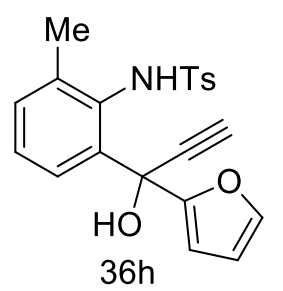

$125 \mathrm{MHz}, \mathrm{CDCl} 3$

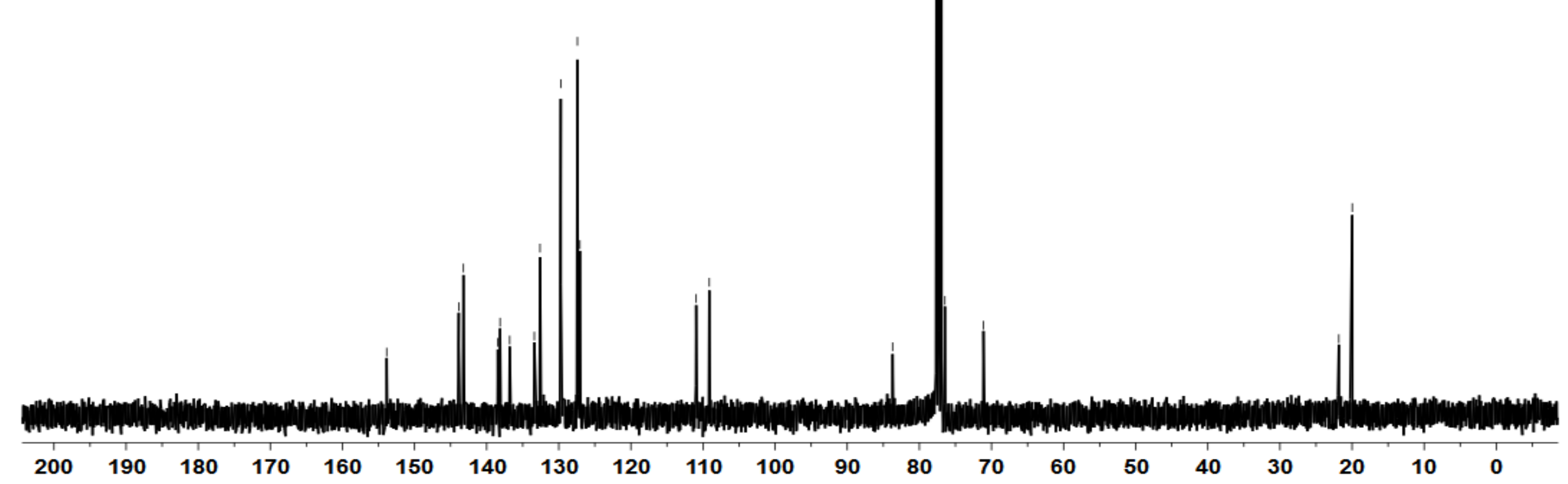




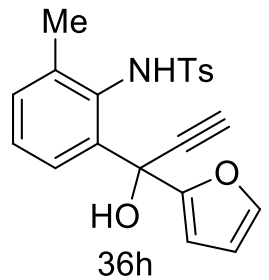

$125 \mathrm{MHz}, \mathrm{CDCl} 3$

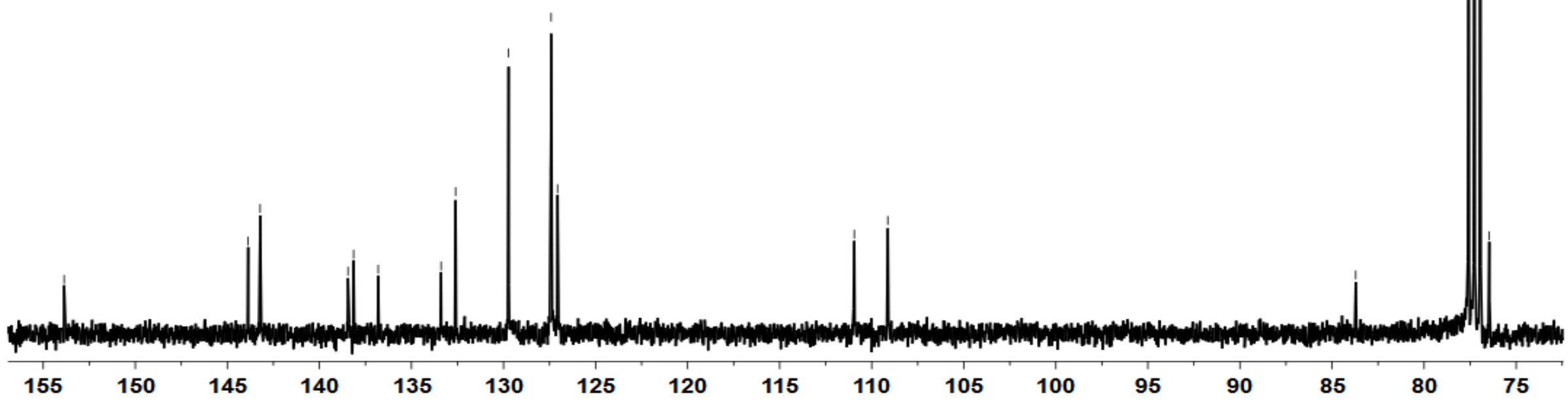




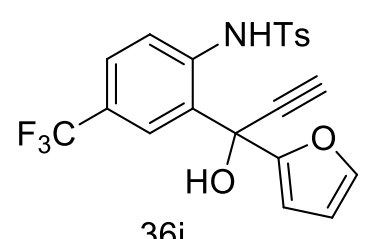

$400 \mathrm{MHz}, \mathrm{CDCl} 3$

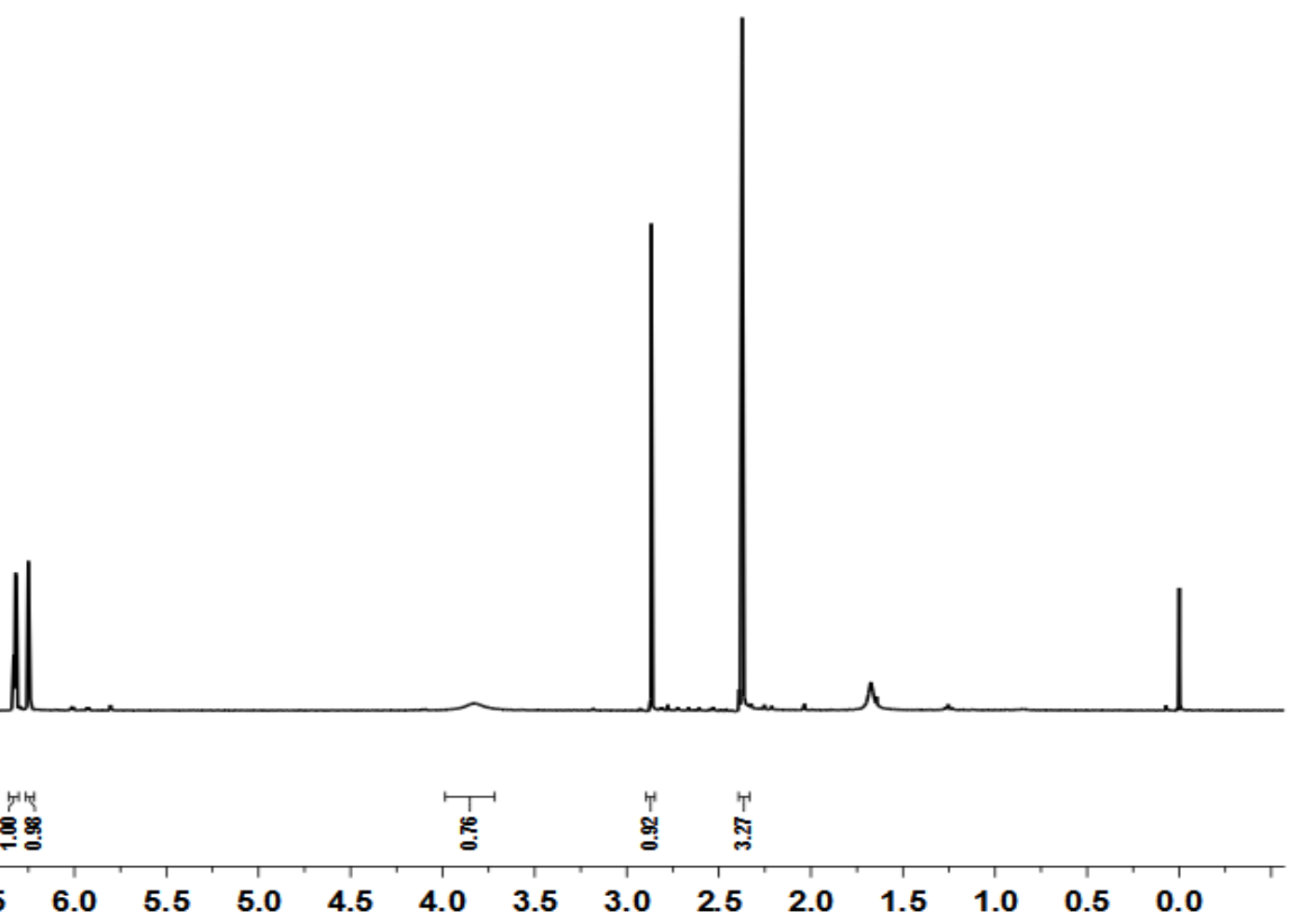




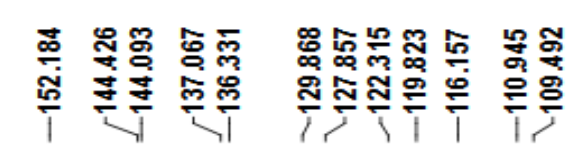

통 웅

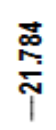

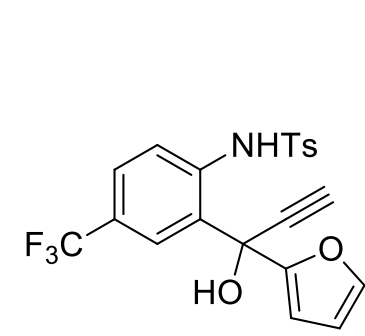

$36 \mathrm{i}$

$100 \mathrm{MHz}, \mathrm{CDCl} 3$

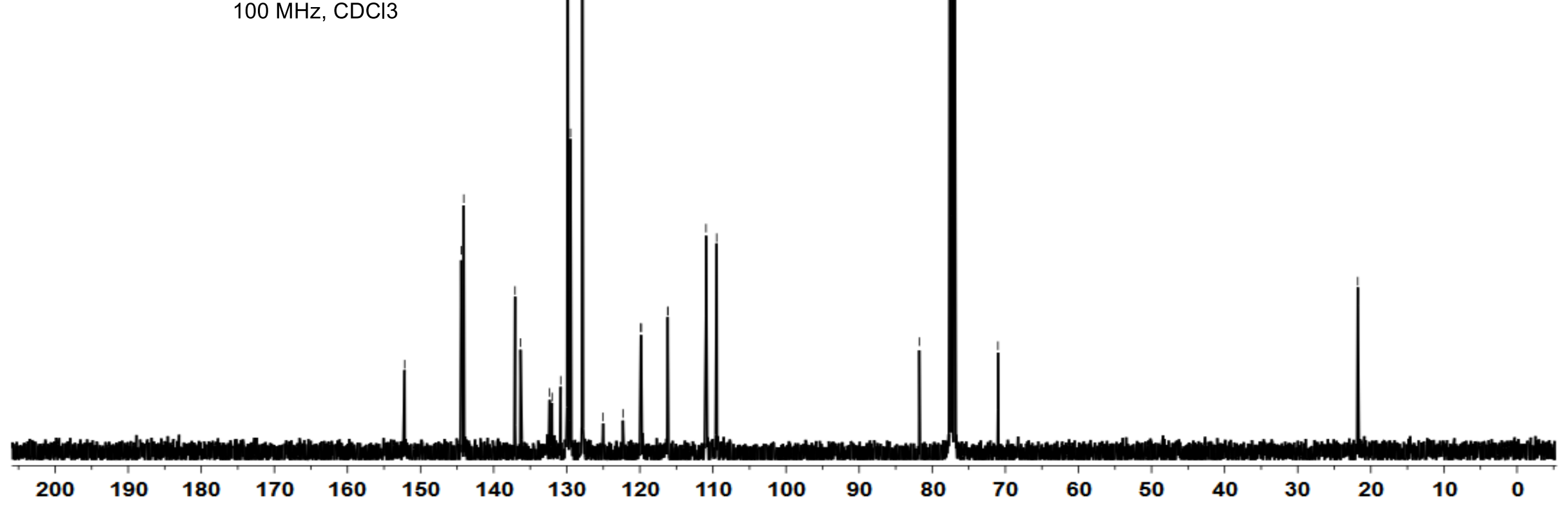




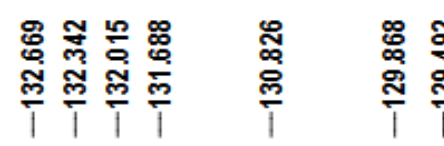

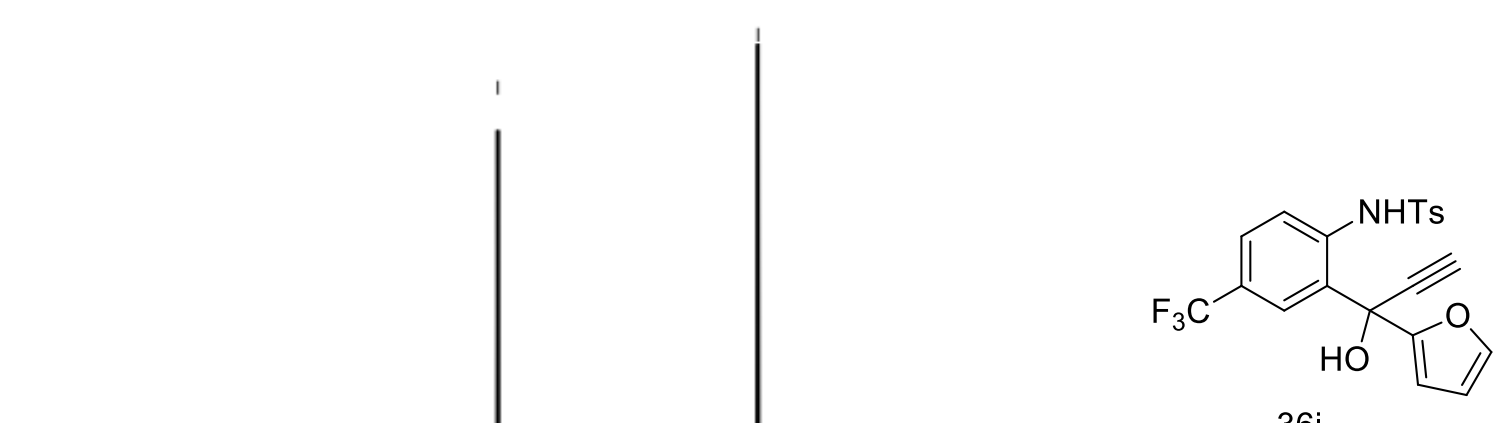

36

$100 \mathrm{MHz}, \mathrm{CDCl} 3$

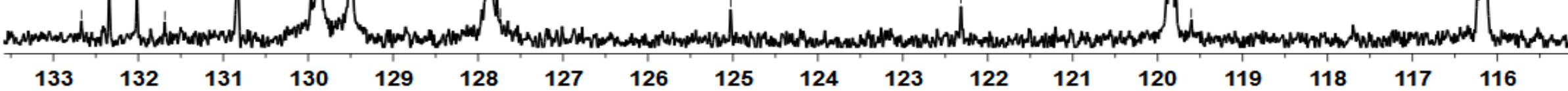




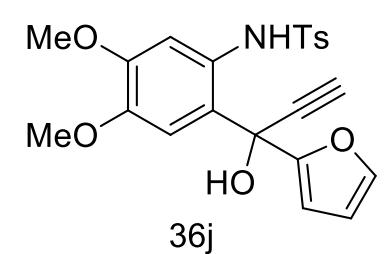

$500 \mathrm{MHz}, \mathrm{CDCl} 3$

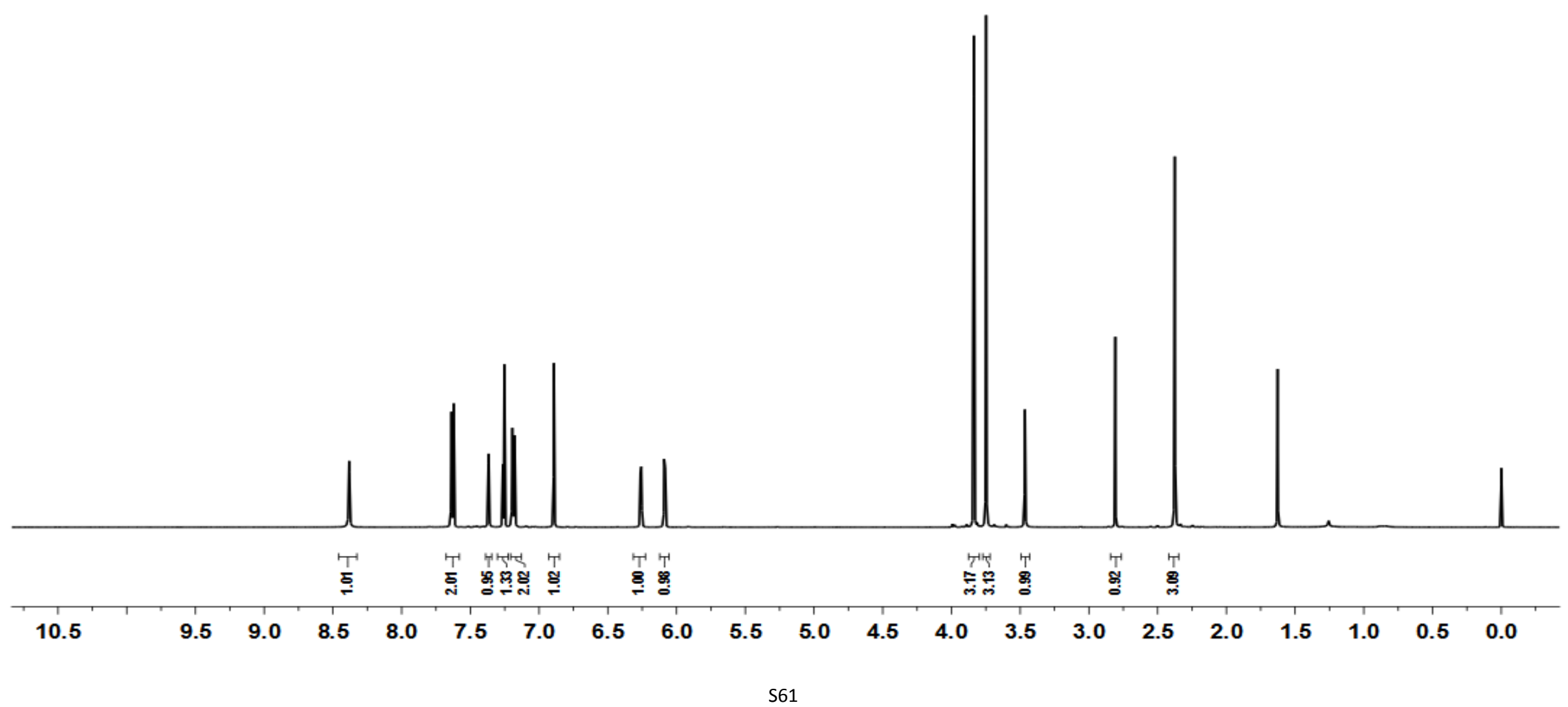




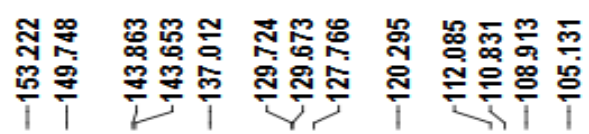

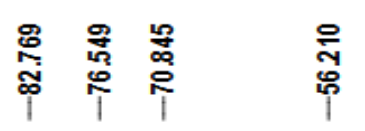

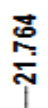

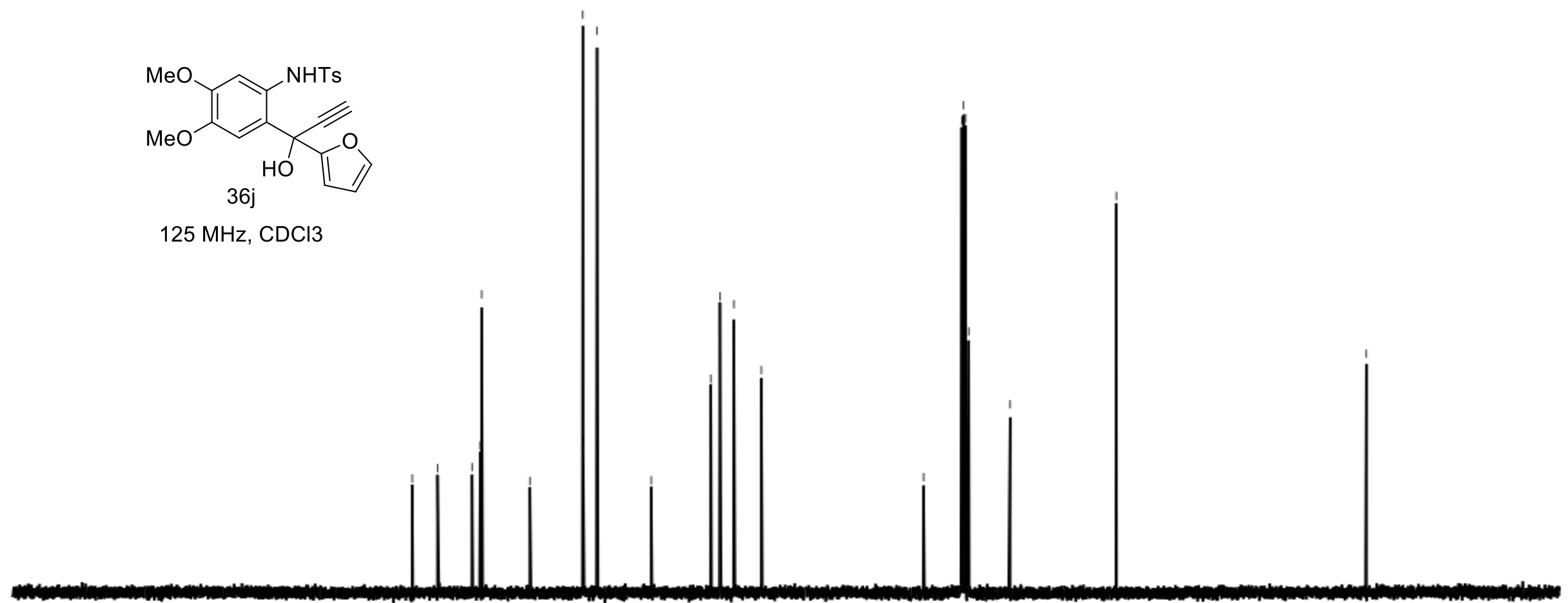



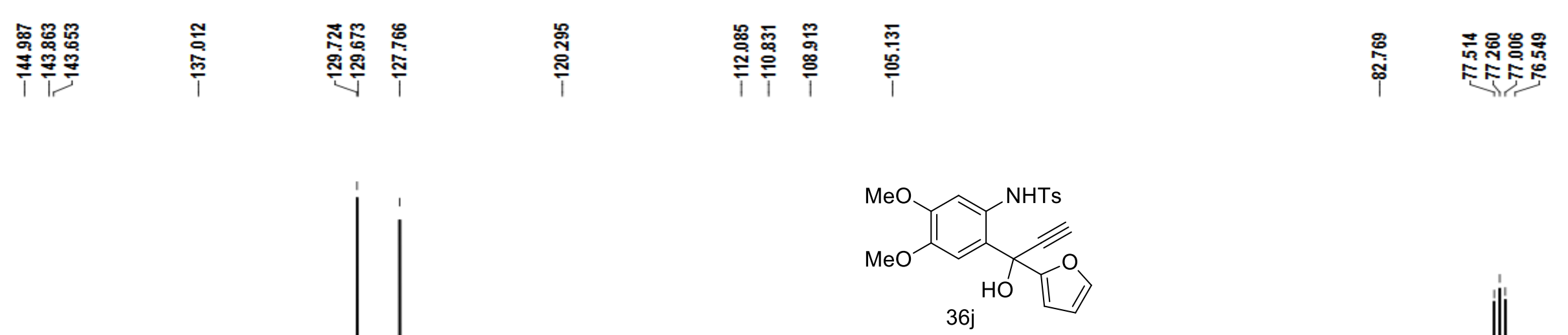

$125 \mathrm{MHz}, \mathrm{CDCl} 3$

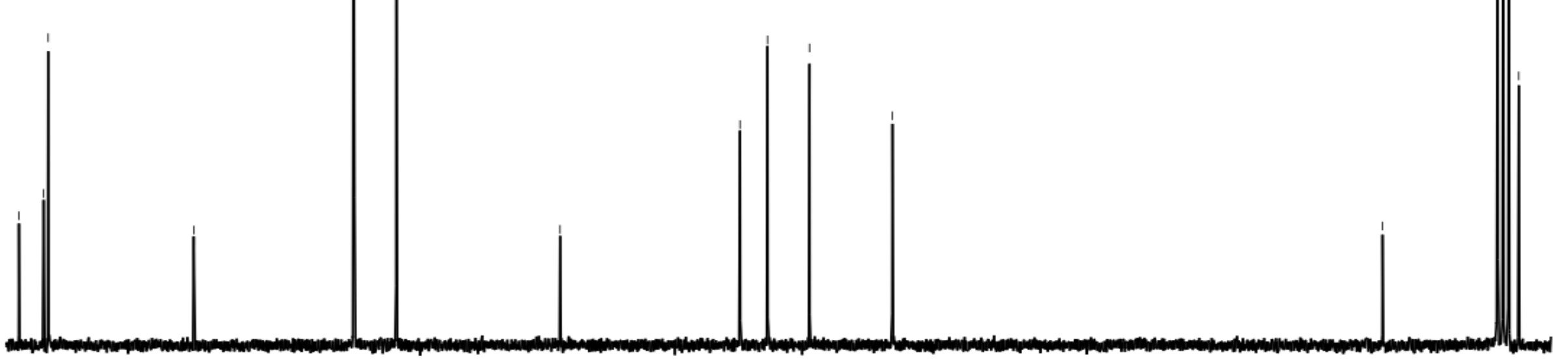

130

125

120

115

110

105

100

95

90

85

80 


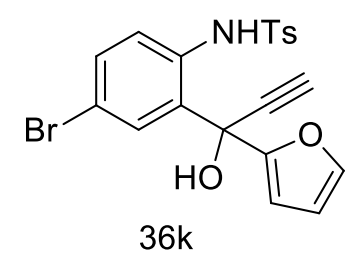

$500 \mathrm{MHz}, \mathrm{CDCl} 3$

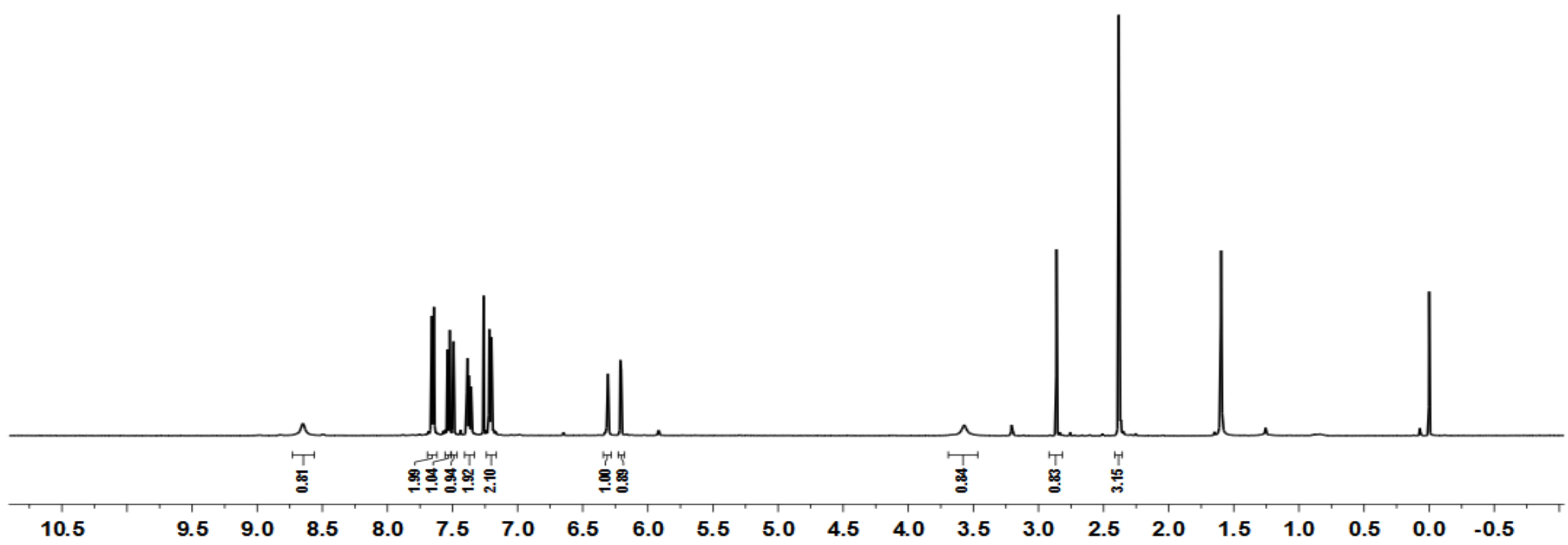




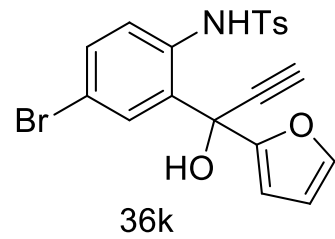

$100 \mathrm{MHz}, \mathrm{CDCl} 3$

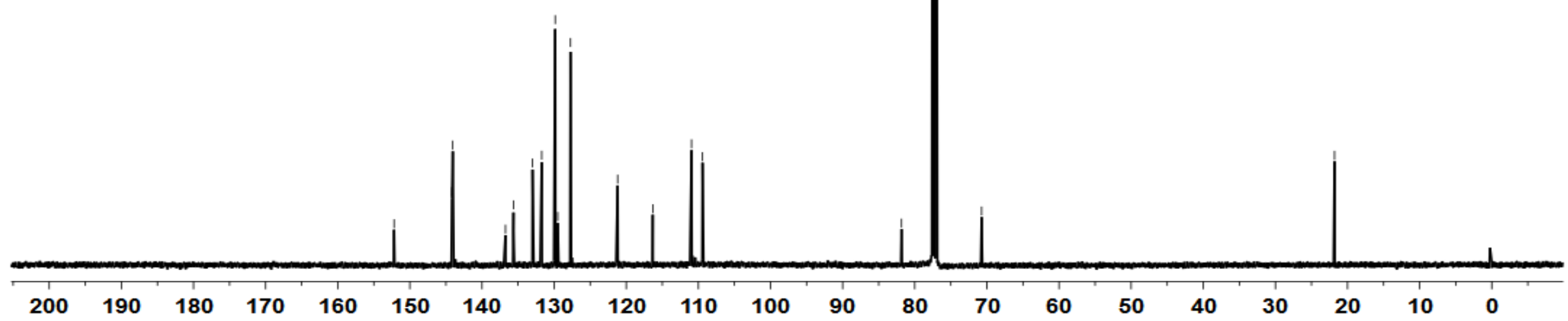




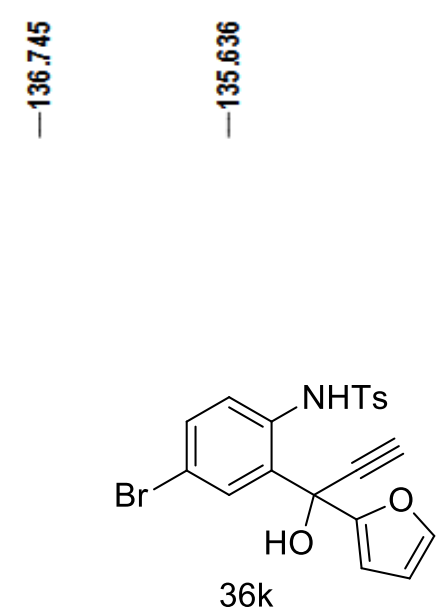

$100 \mathrm{MHz}, \mathrm{CDCl} 3$

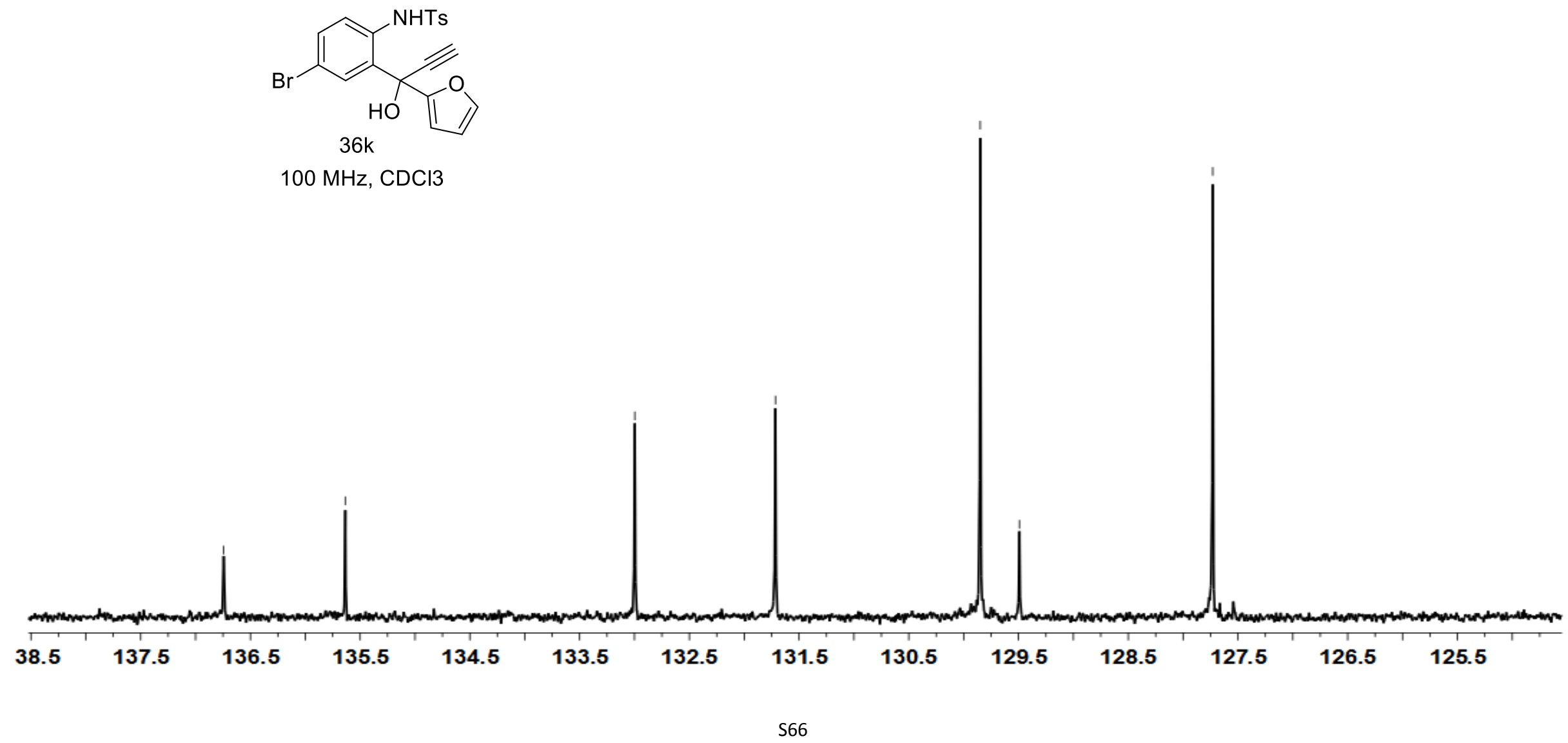




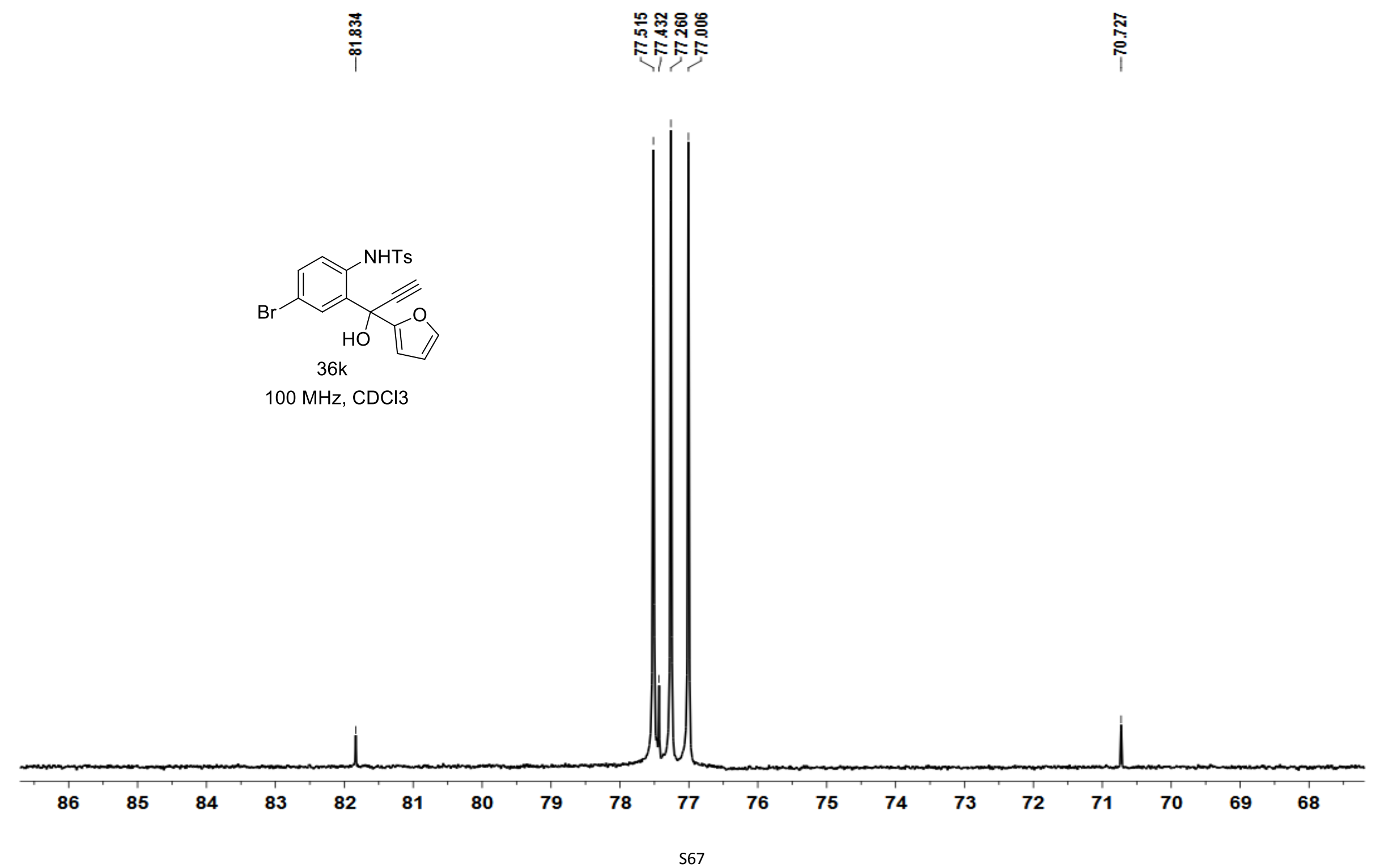




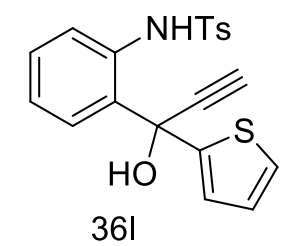

$400 \mathrm{MHz}, \mathrm{CDCl} 3$

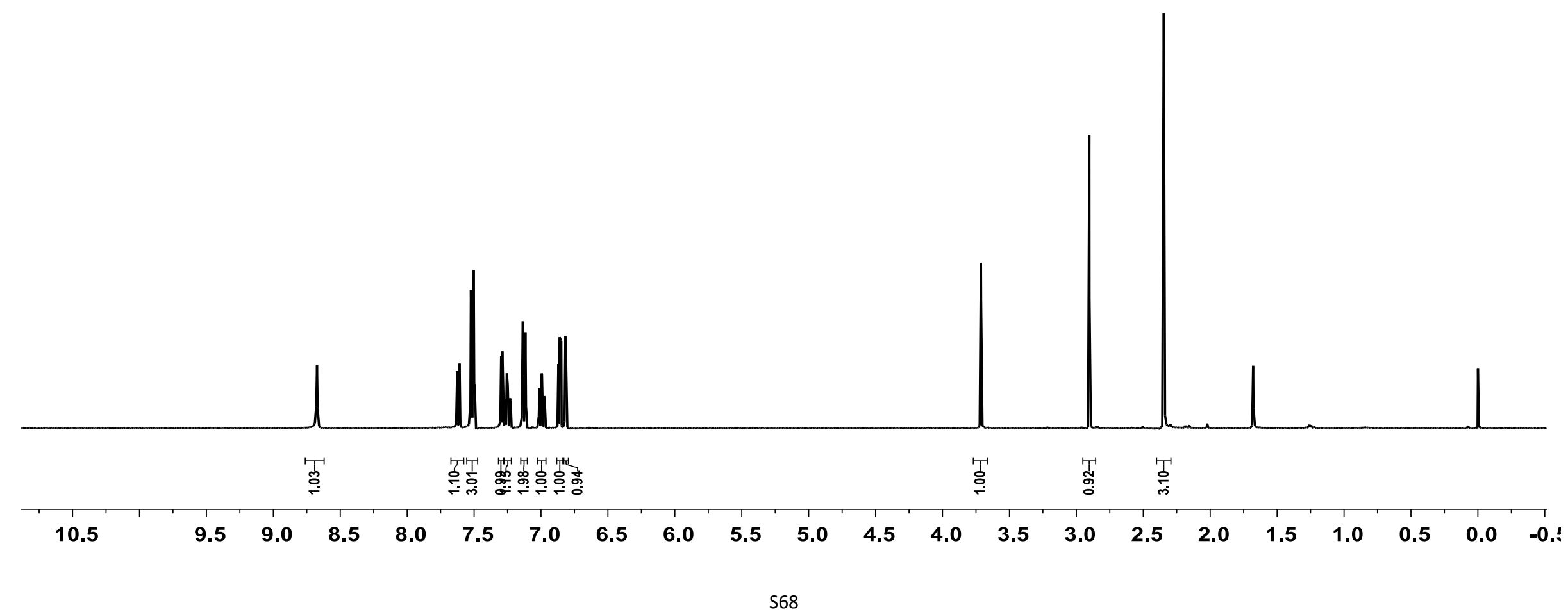




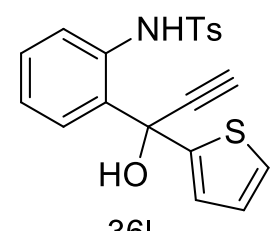

361

$100 \mathrm{MHz}, \mathrm{CDCl} 3$

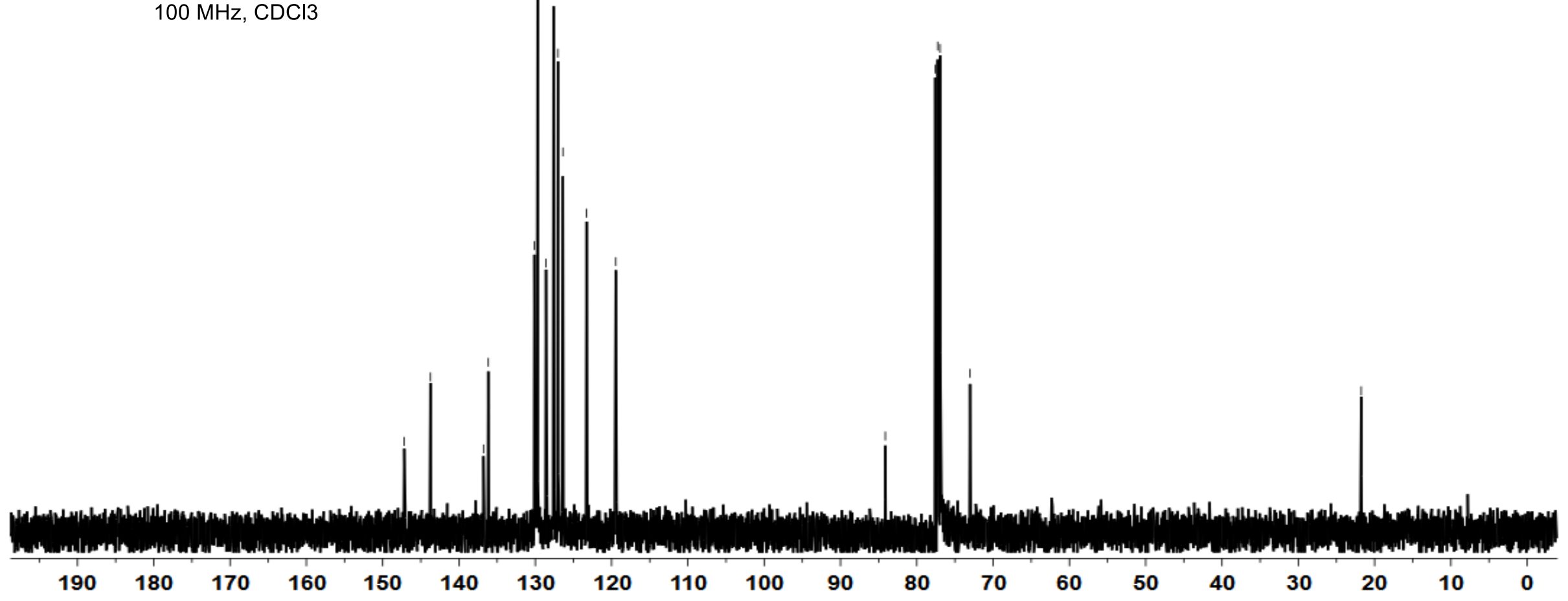




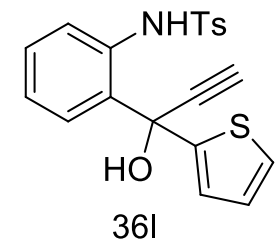

$100 \mathrm{MHz}, \mathrm{CDCl} 3$

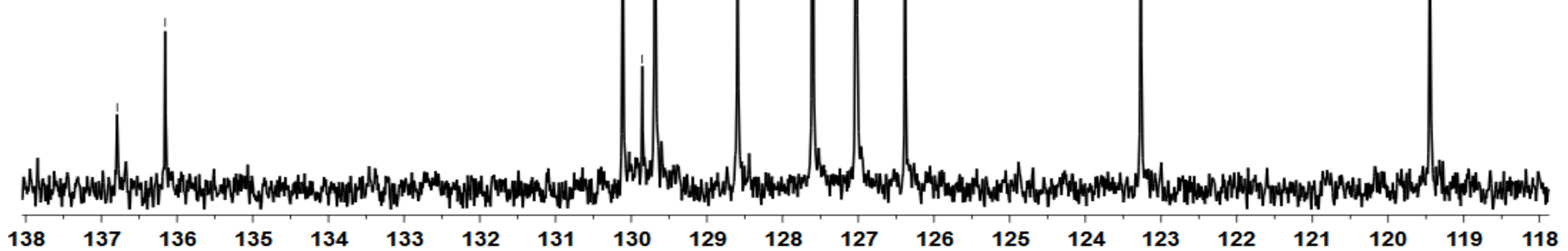




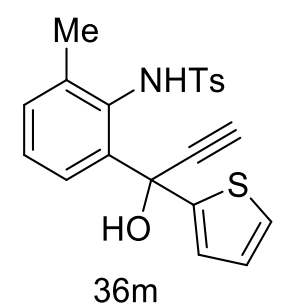

$400 \mathrm{MHz}, \mathrm{CD} 3 \mathrm{OD}$

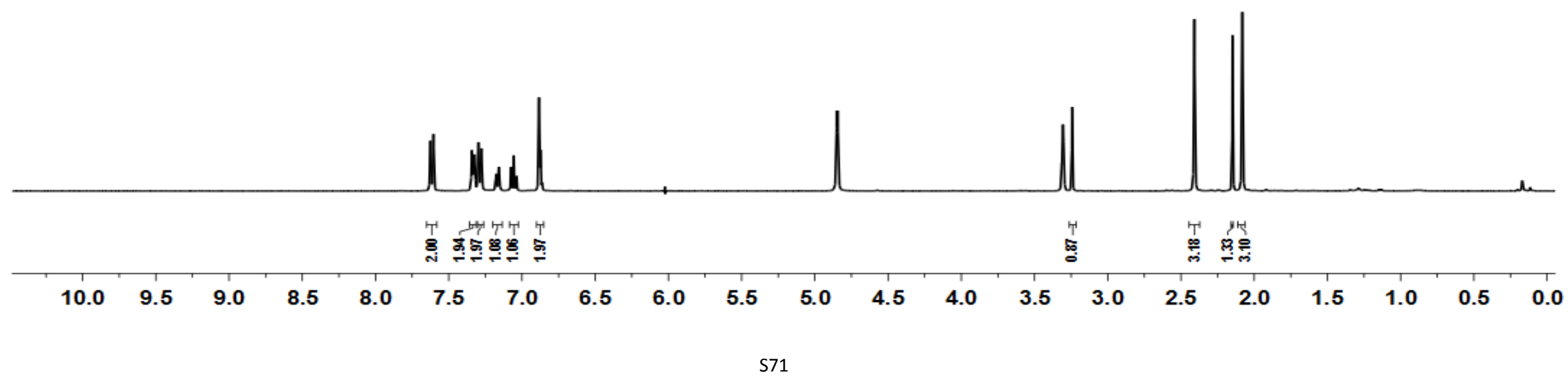




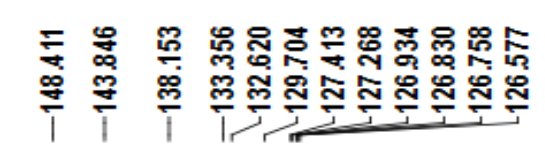

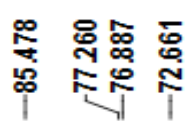

옹

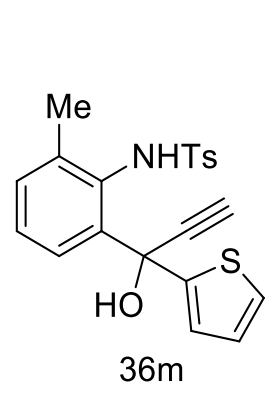

$100 \mathrm{MHz}, \mathrm{CDCl} 3$

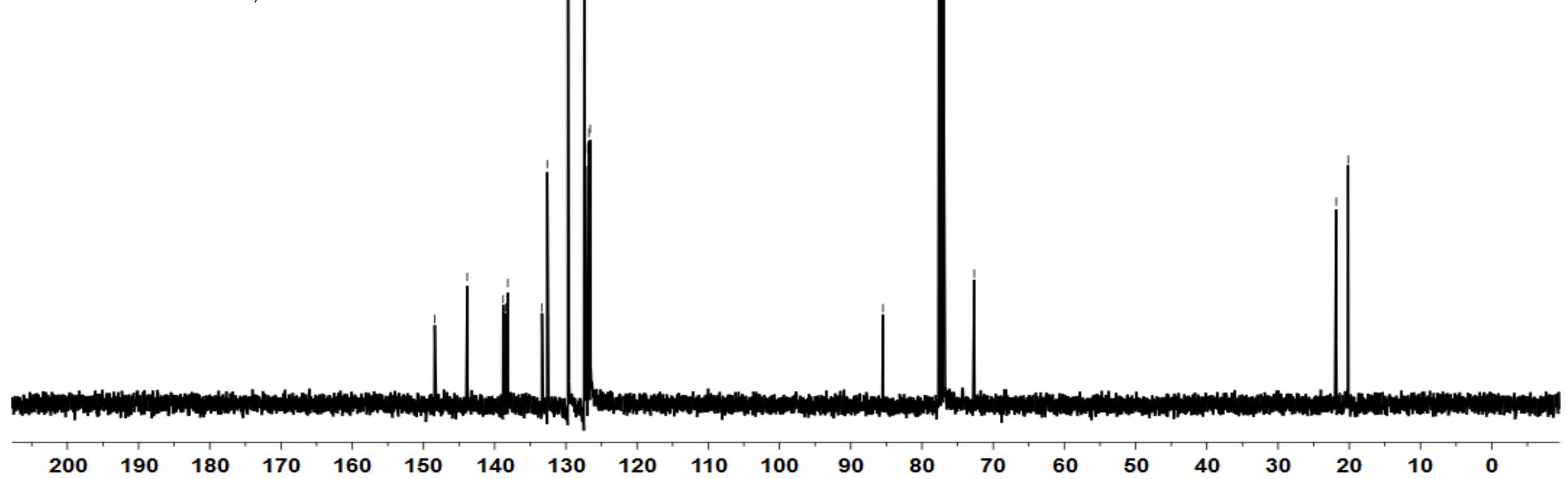




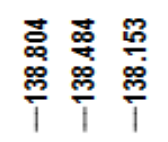

$100 \mathrm{MHz}, \mathrm{CDCl} 3$

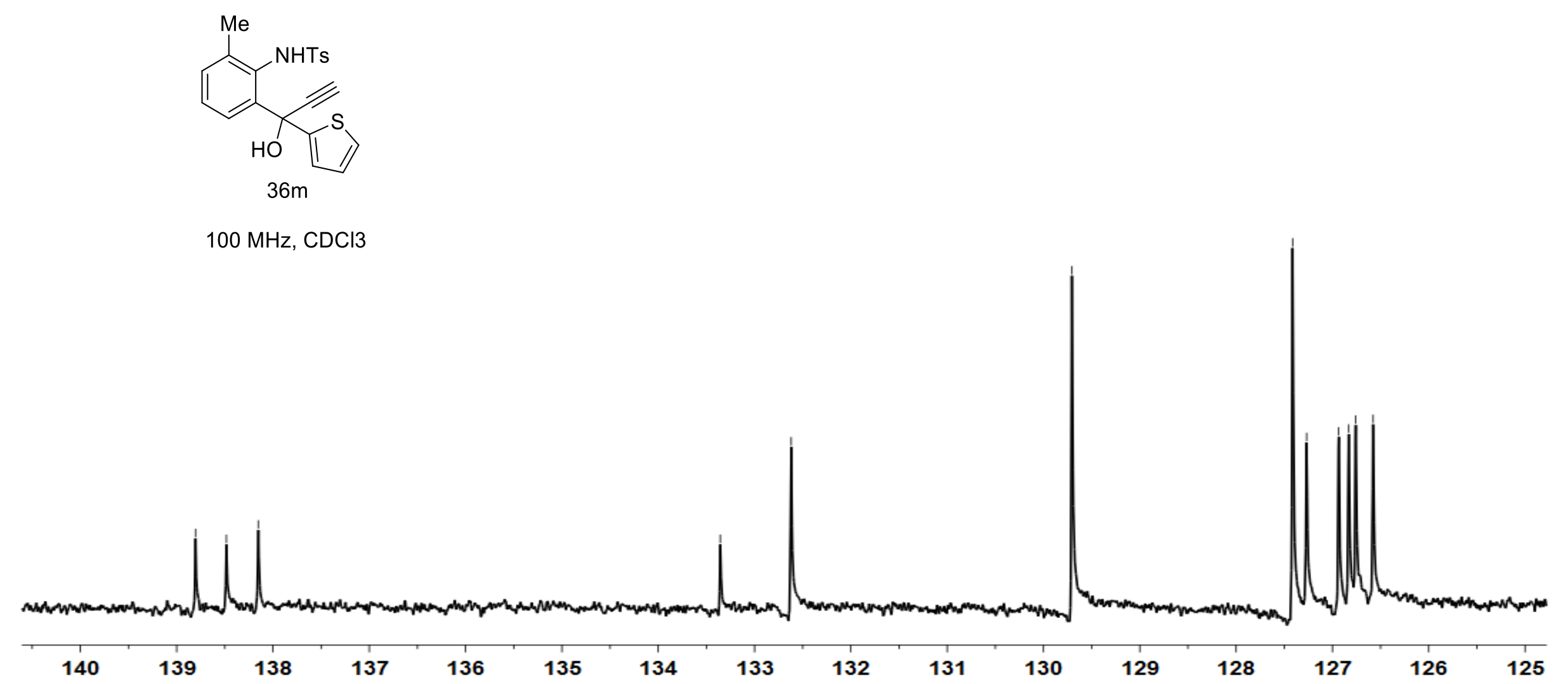

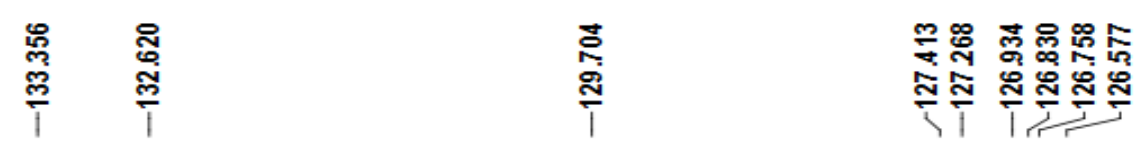




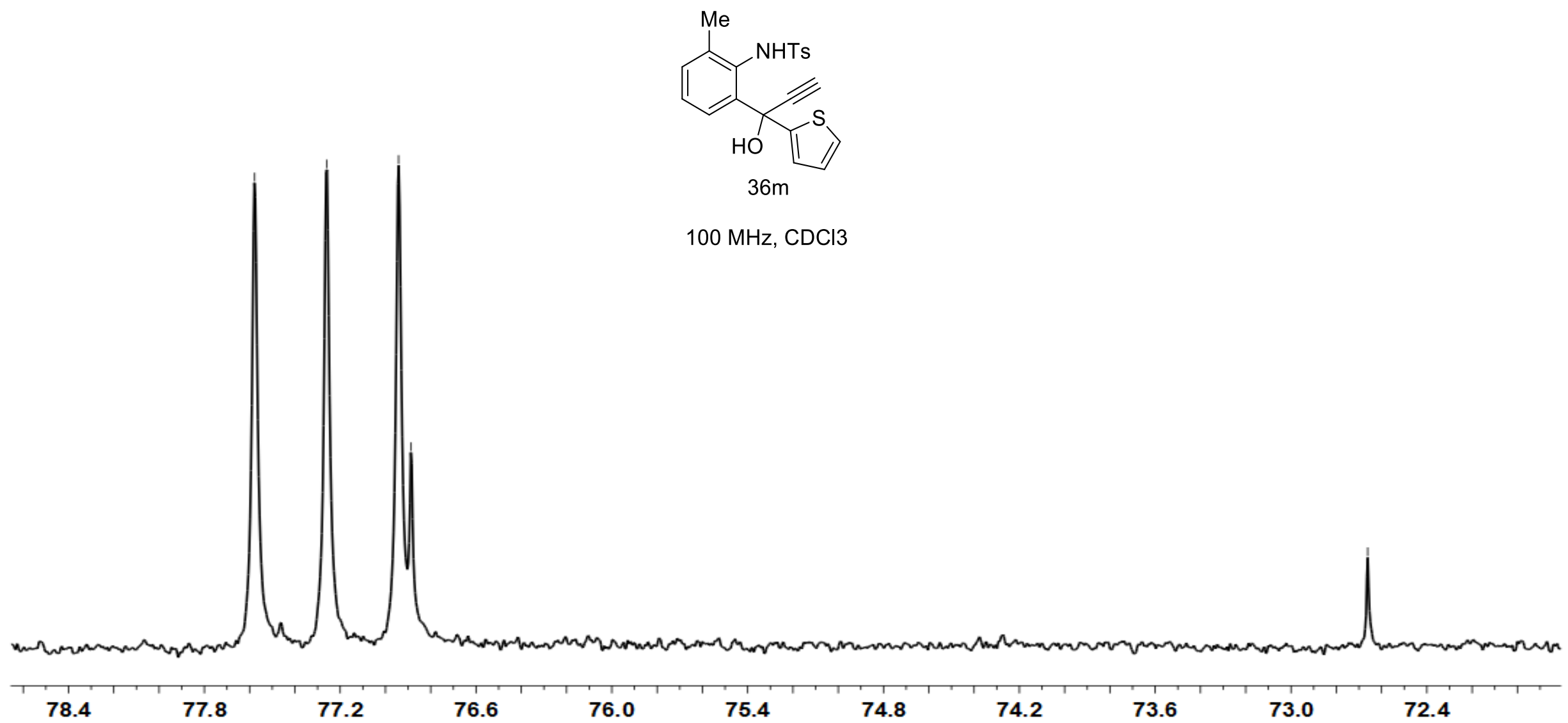




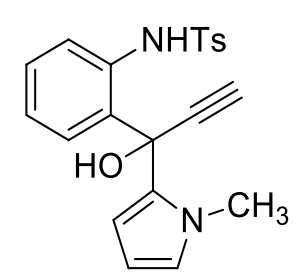

$36 n$

$500 \mathrm{MHz},(C D 3) 2 \mathrm{CO}$

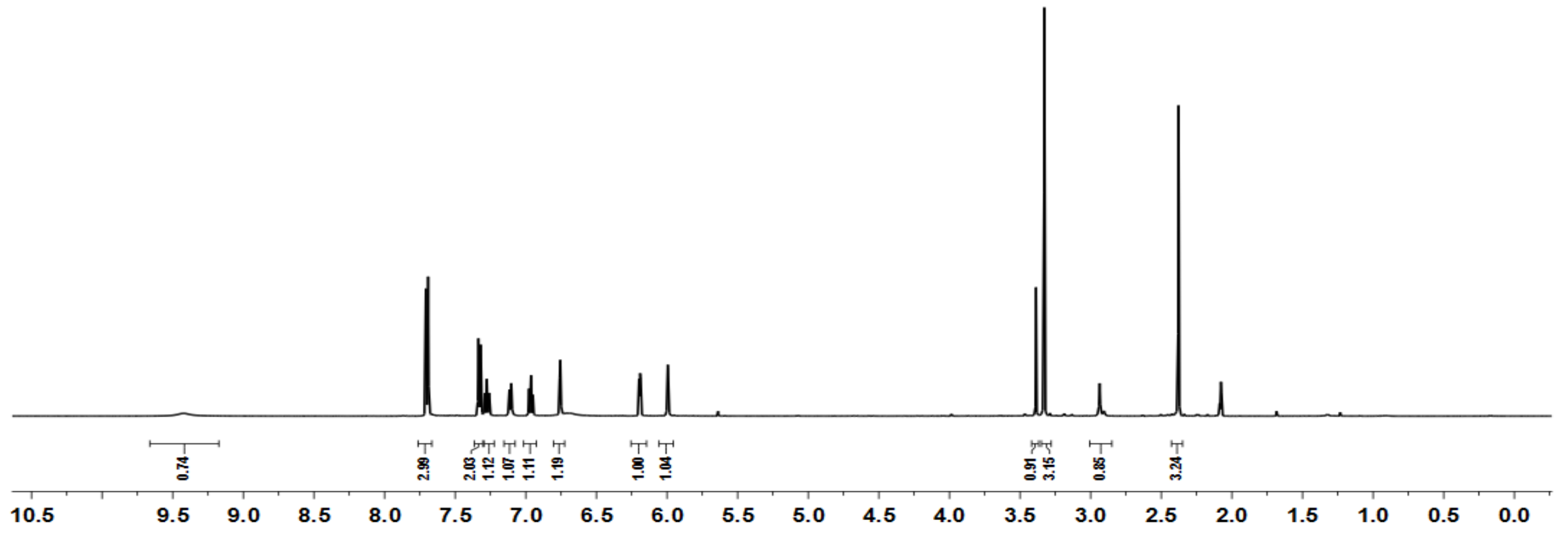



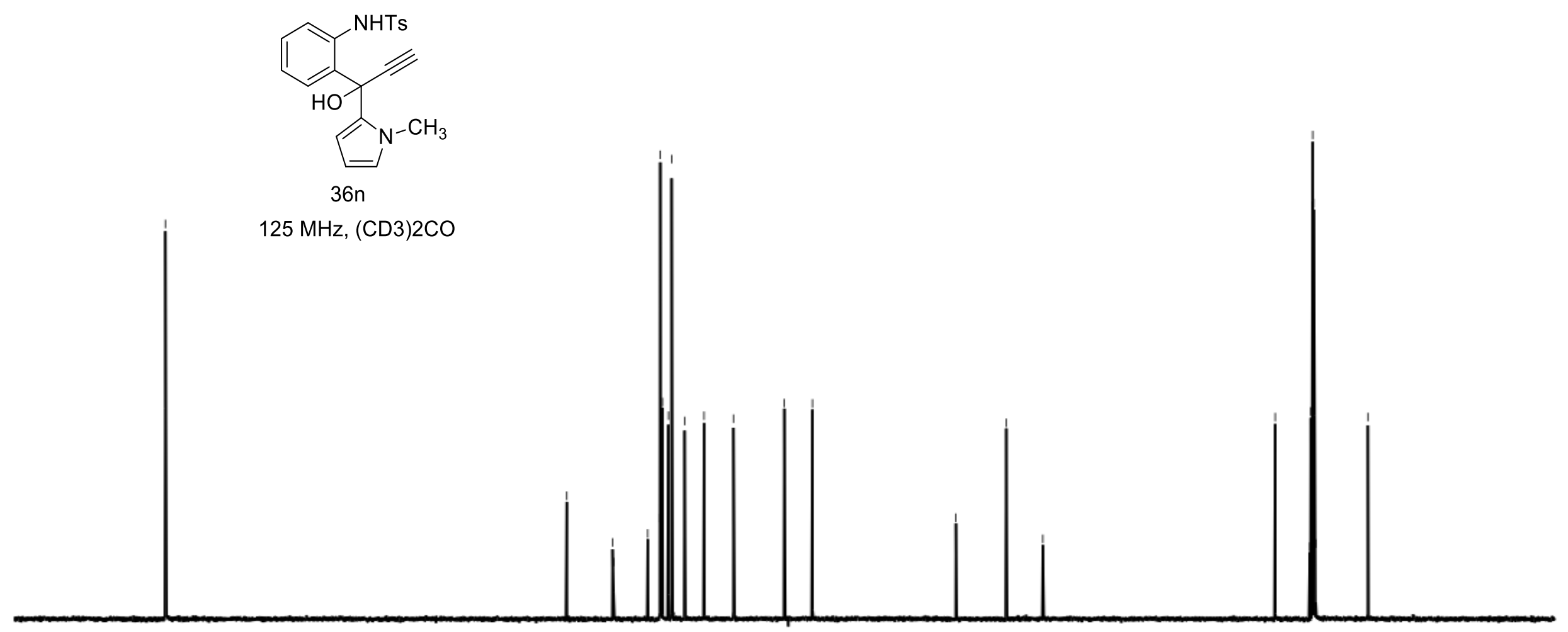
군

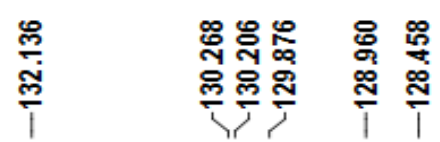

$\stackrel{8}{\stackrel{\circ}{\circ}}$

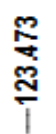

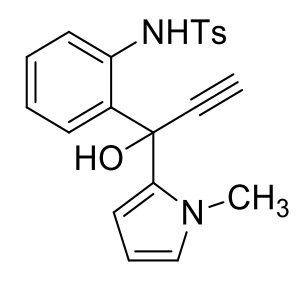

$36 \mathrm{n}$

$125 \mathrm{MHz},(\mathrm{CD} 3) 2 \mathrm{CO}$

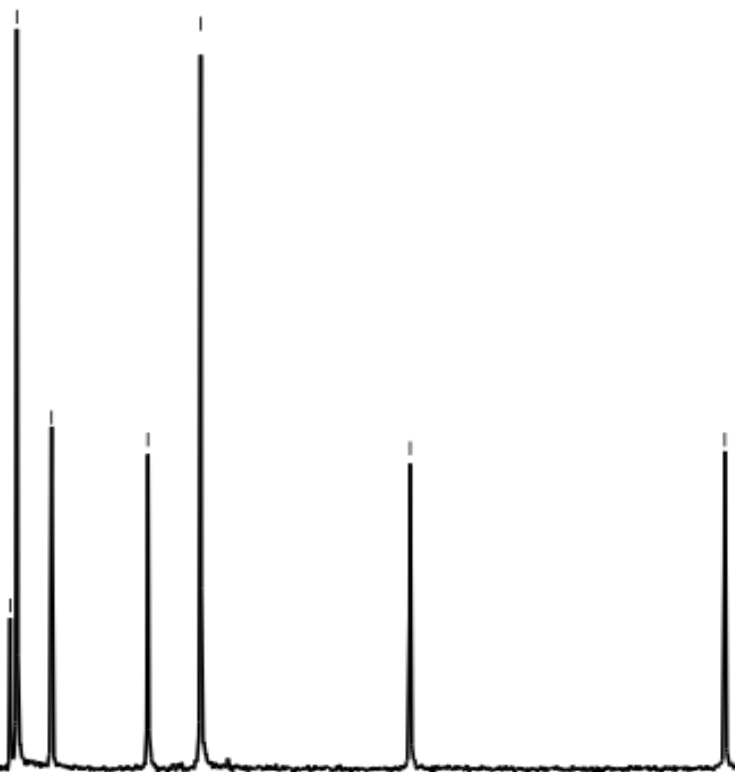




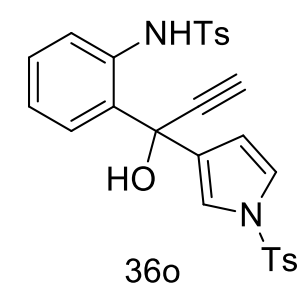

$500 \mathrm{MHz}, \mathrm{CDCl} 3$

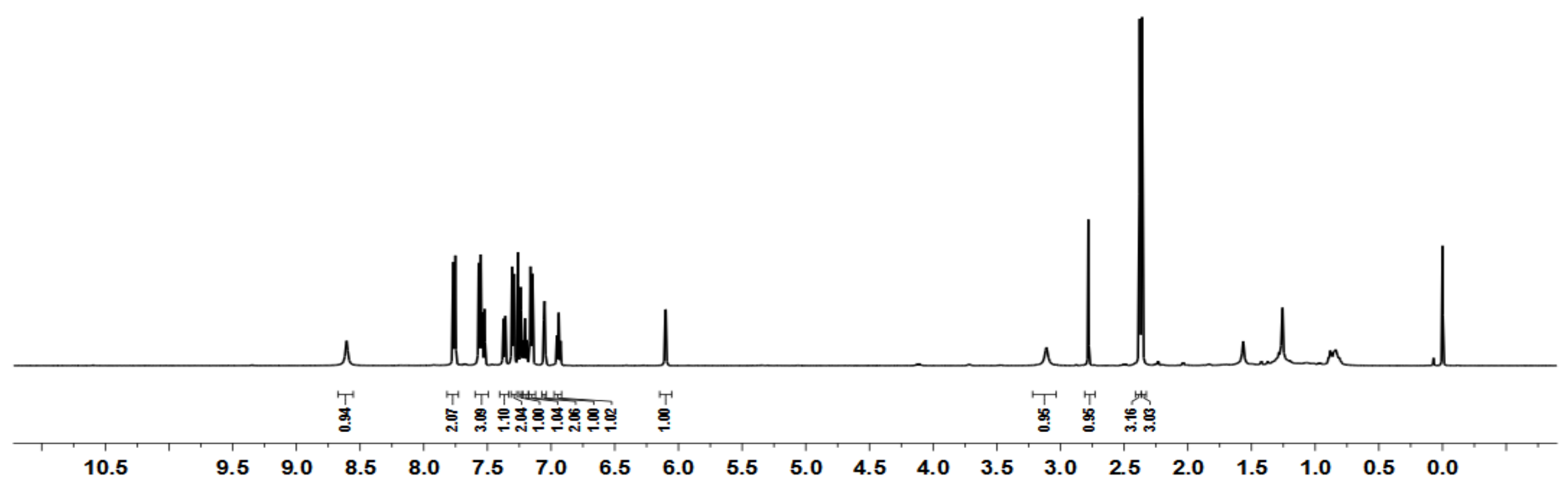




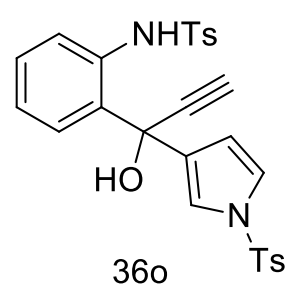

$500 \mathrm{MHz}, \mathrm{CDCl} 3$

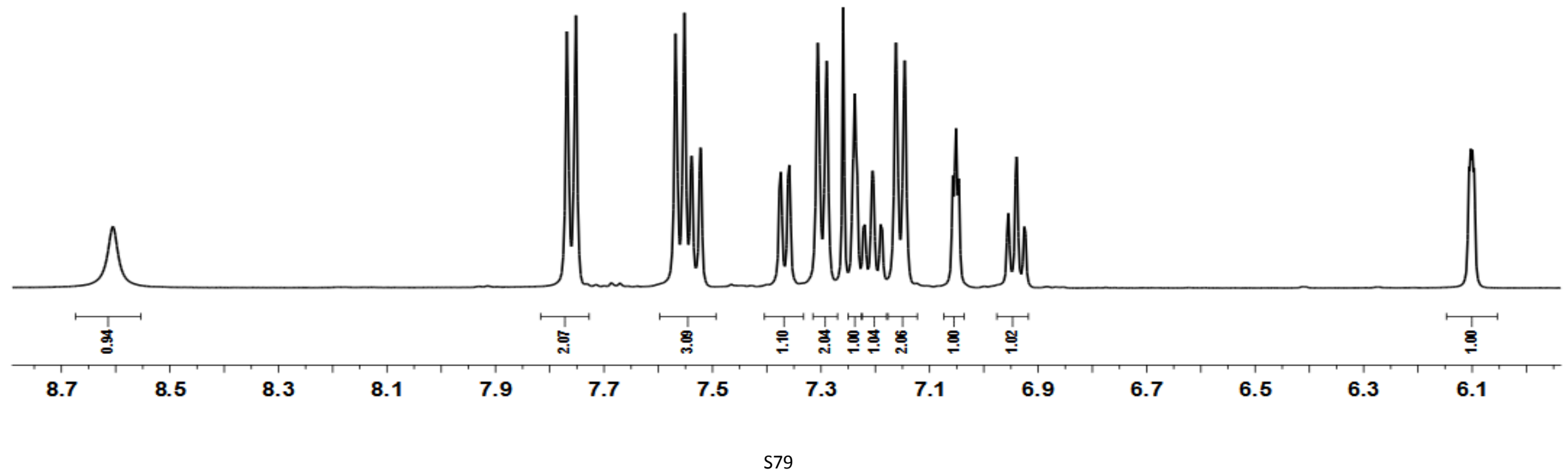




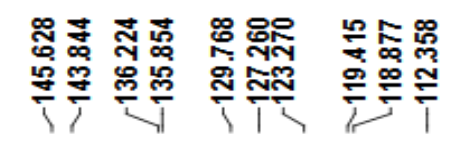

占 品品

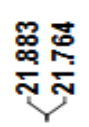

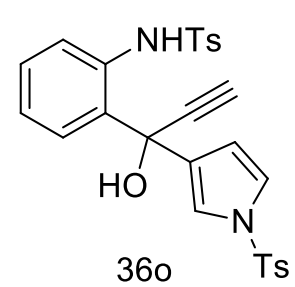

$100 \mathrm{MHz}, \mathrm{CDCl} 3$

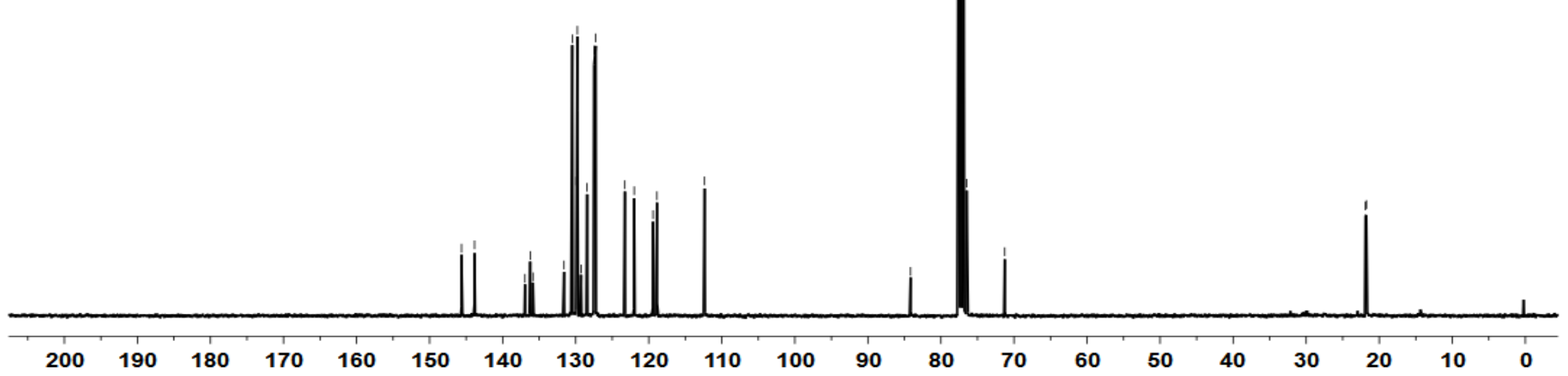




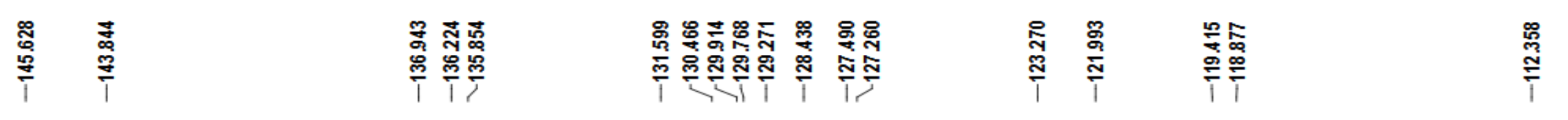

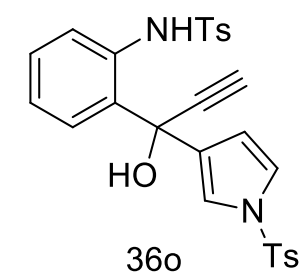

$100 \mathrm{MHz}, \mathrm{CDCl} 3$

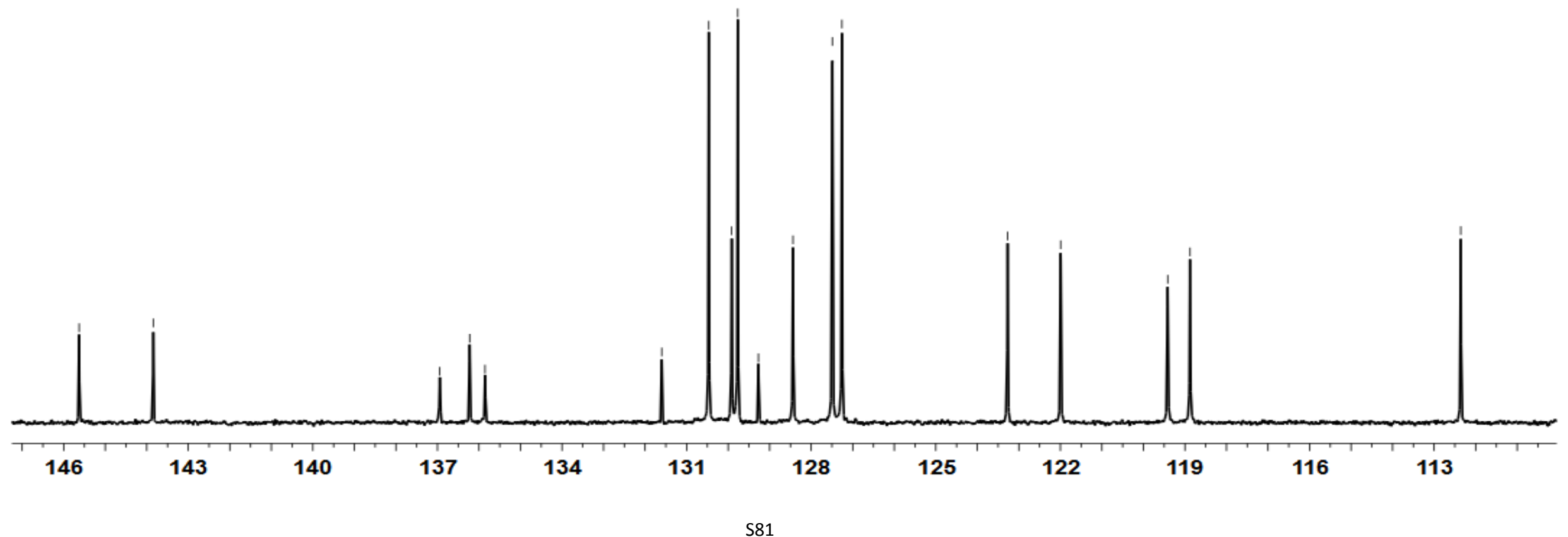




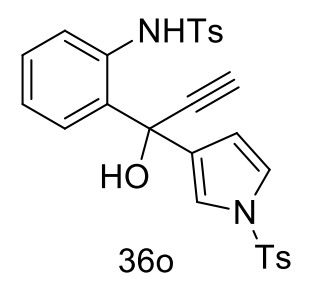

$100 \mathrm{MHz}, \mathrm{CDCl} 3$

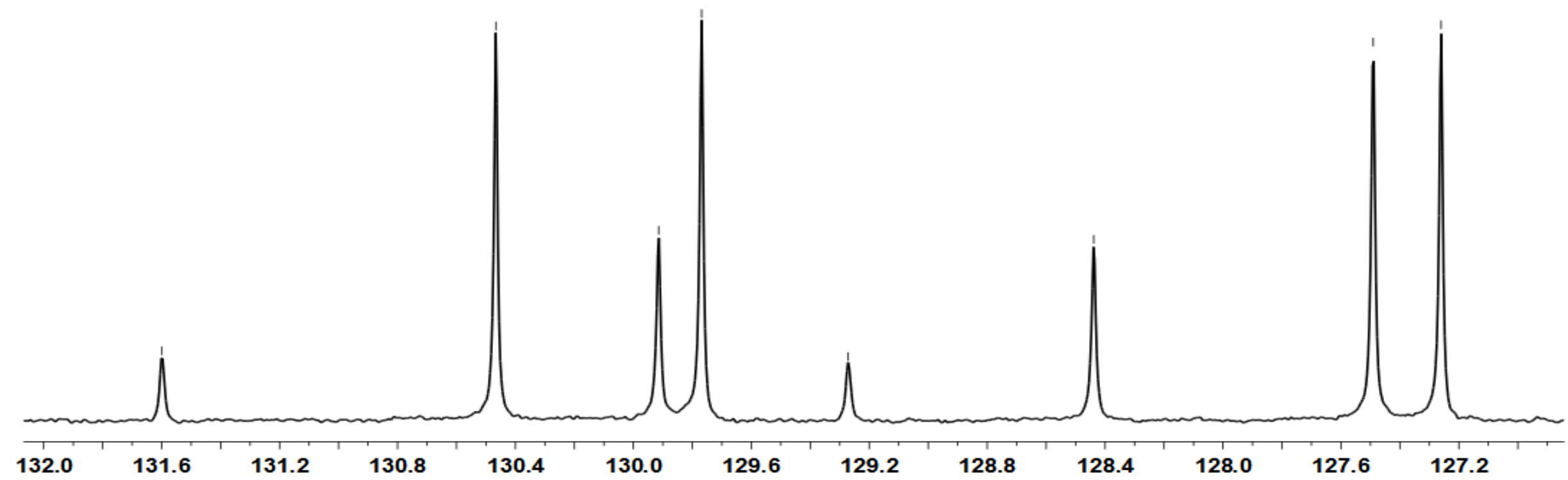




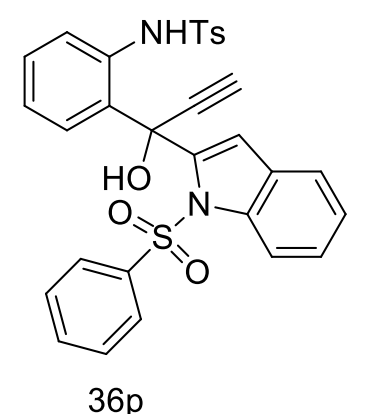

$500 \mathrm{MHz}, \mathrm{CDCl} 3$

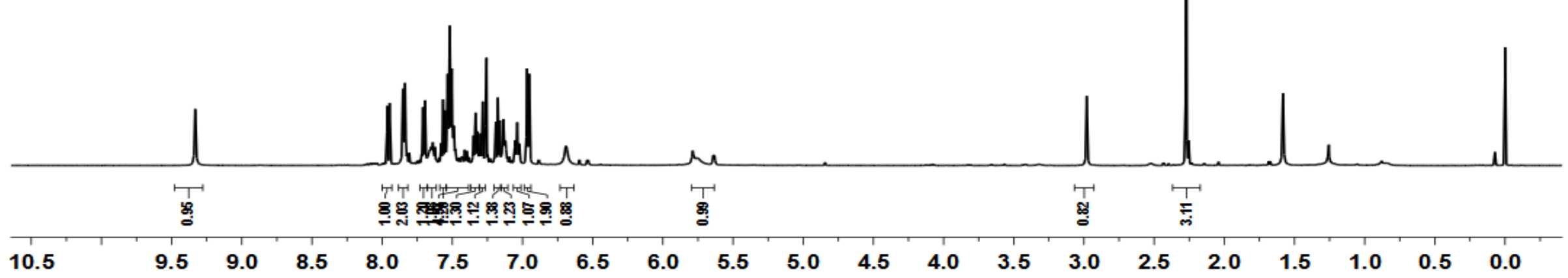




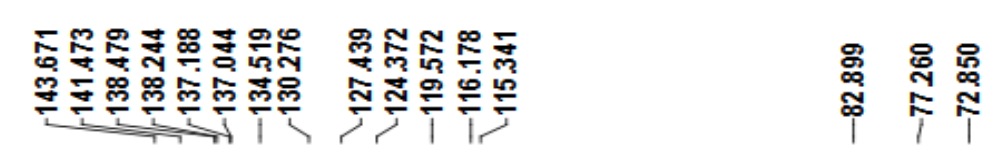

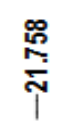

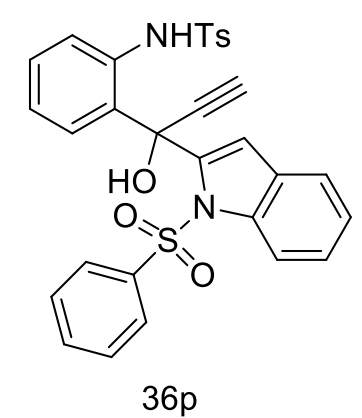

$100 \mathrm{MHz}, \mathrm{CDCl} 3$
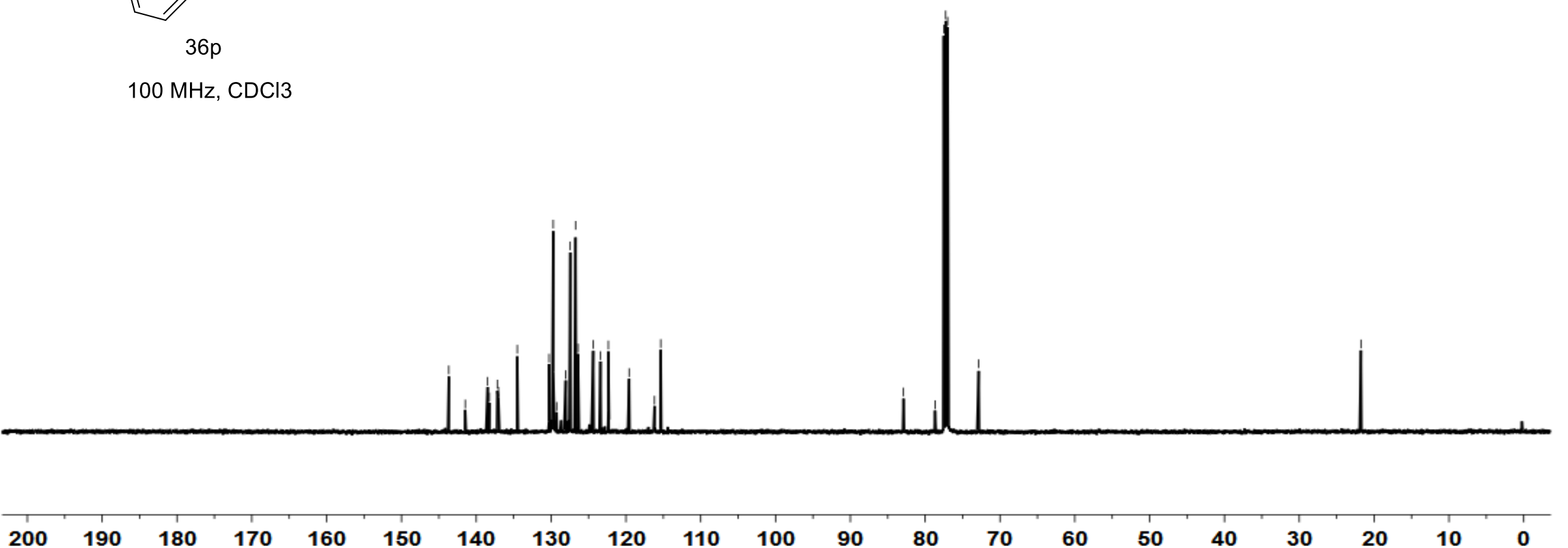

100

90

80 


\begin{tabular}{|c|c|c|c|c|c|}
\hline 疍 & 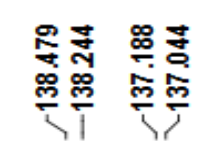 & 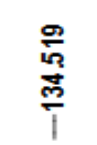 & 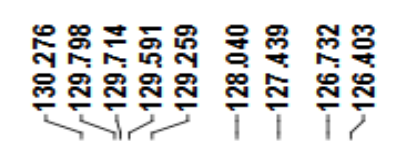 & 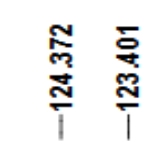 & 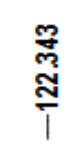 \\
\hline
\end{tabular}

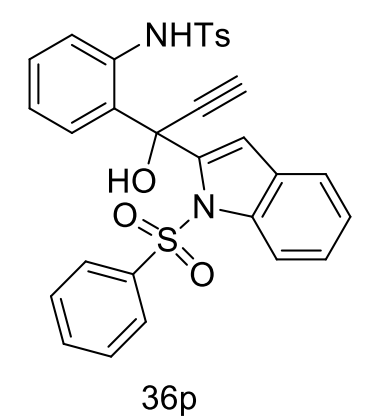

$100 \mathrm{MHz}, \mathrm{CDCl} 3$
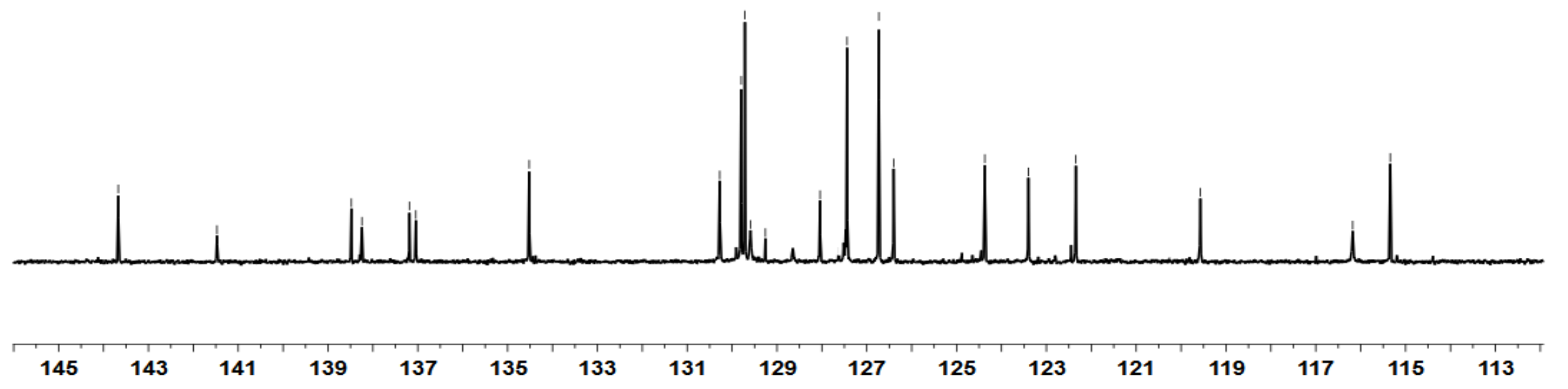

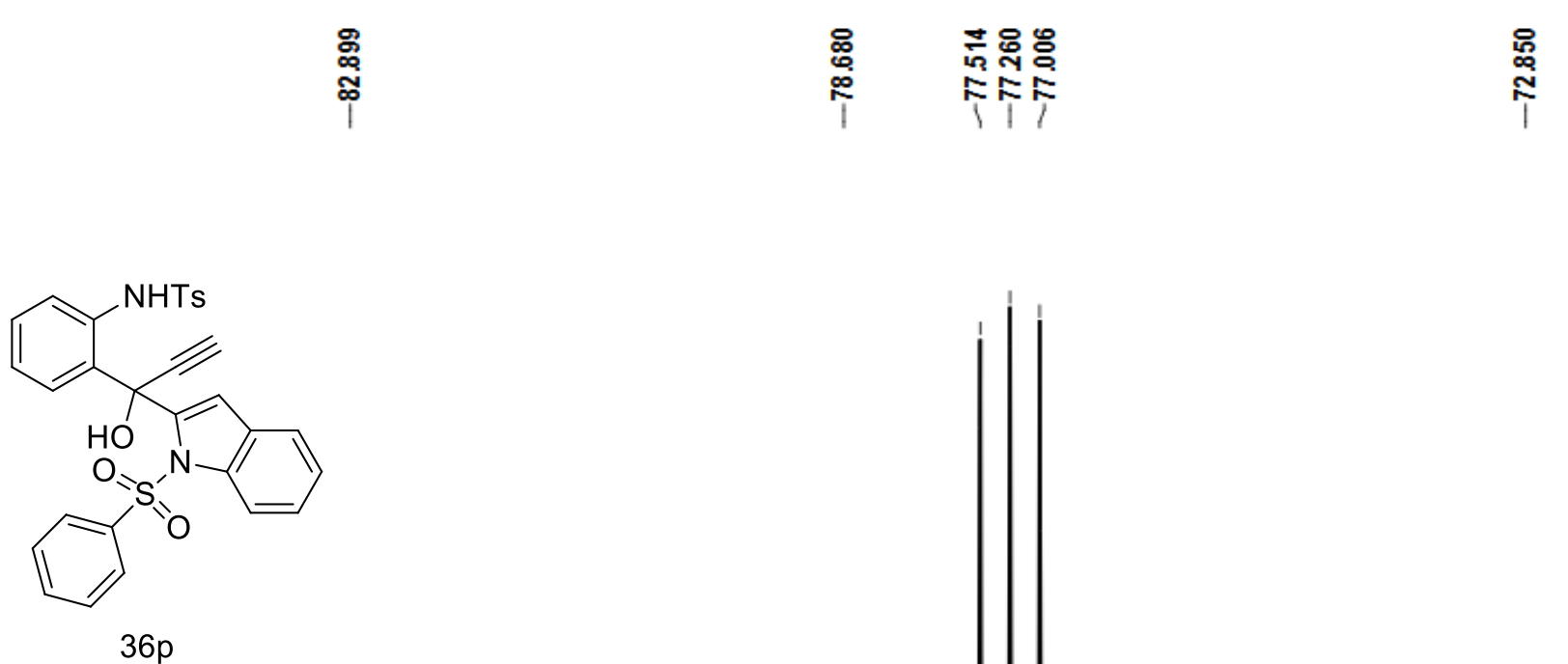

$100 \mathrm{MHz}, \mathrm{CDCl} 3$

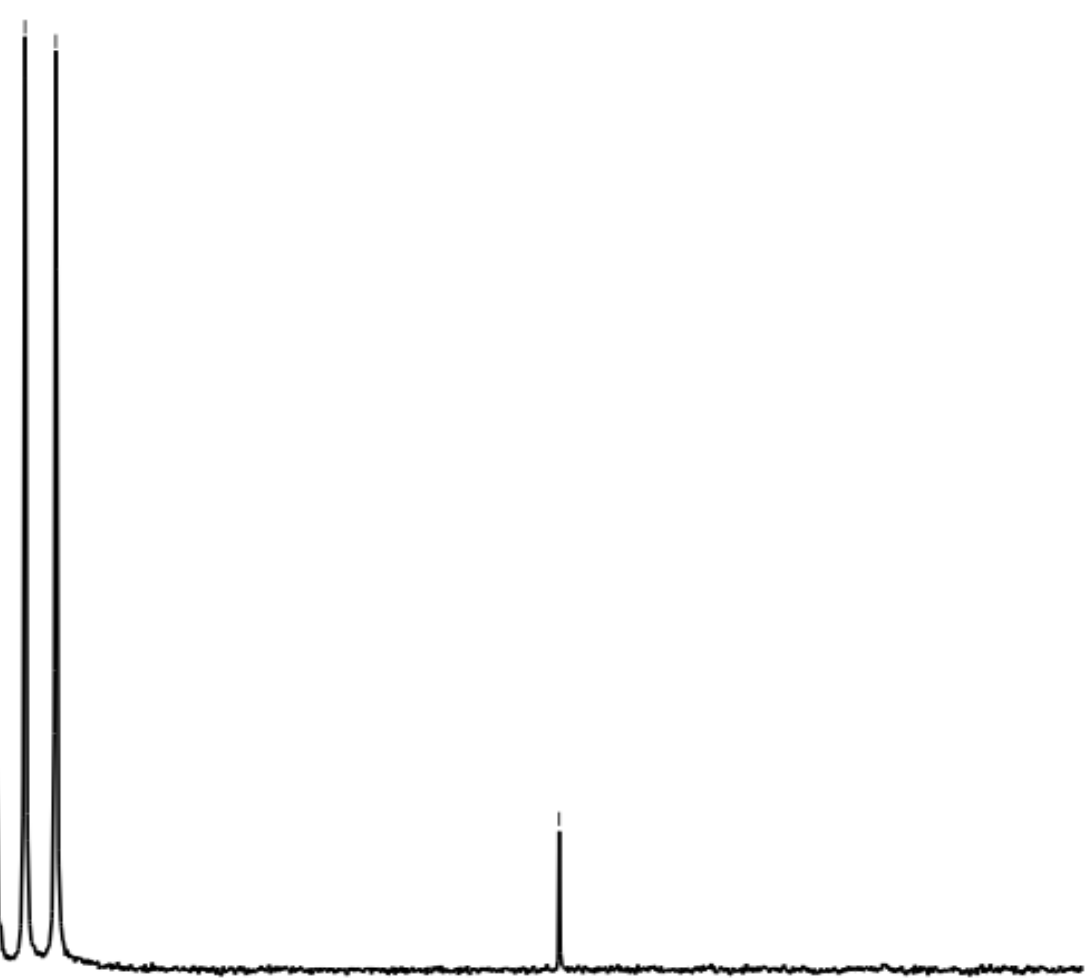




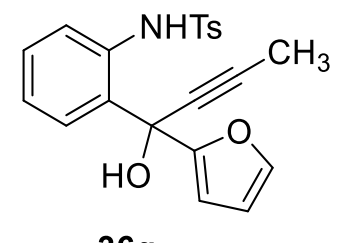

$36 q$

$500 \mathrm{MHz}, \mathrm{CDCl}_{3}$

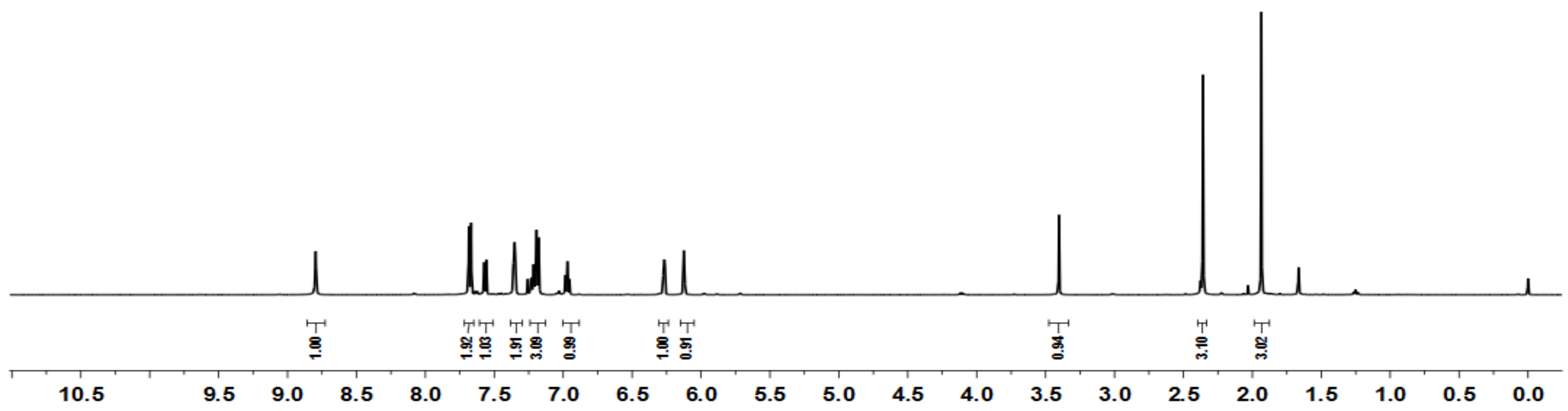




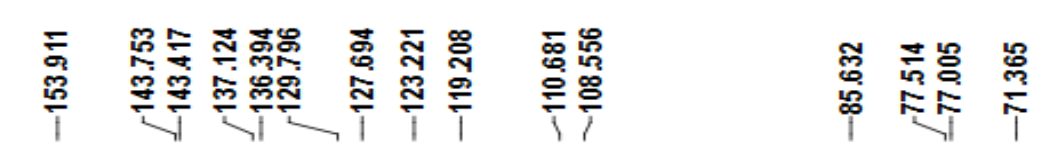

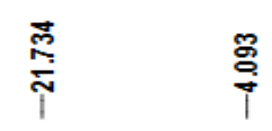

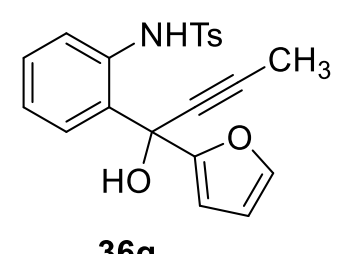

$36 q$

$125 \mathrm{MHz}, \mathrm{CDCl}_{3}$

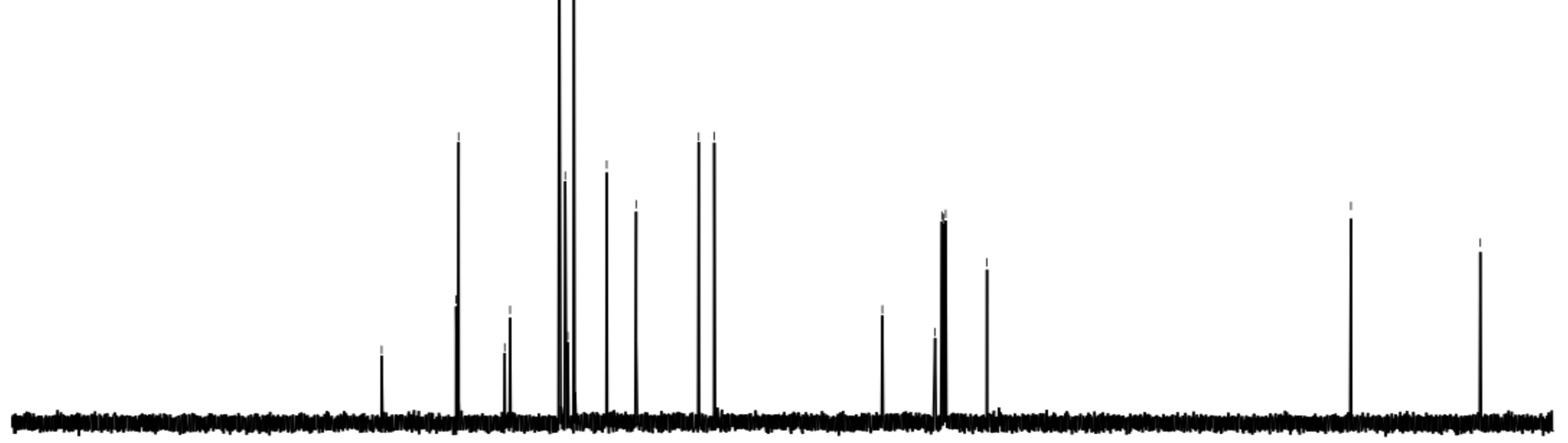

$\begin{array}{lllllllllll}200 & 190 & 180 & 170 & 160 & 150 & 140 & 130 & 120 & 110 & 100\end{array}$
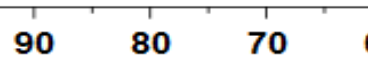

50

40

30

20

10 


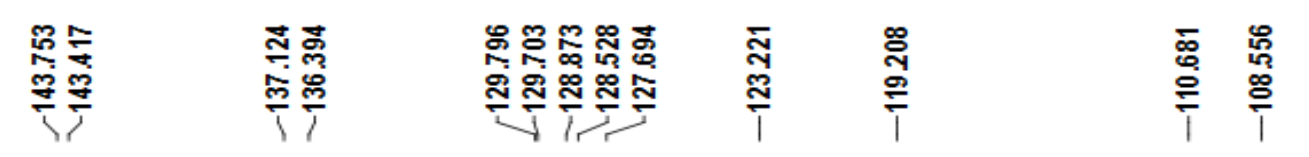

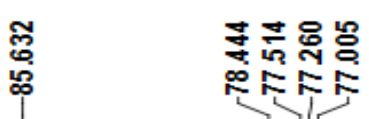

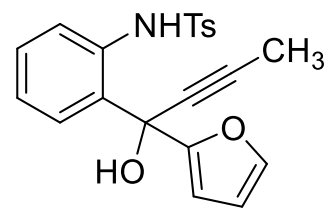

$36 q$

$125 \mathrm{MHz}, \mathrm{CDCl}_{3}$

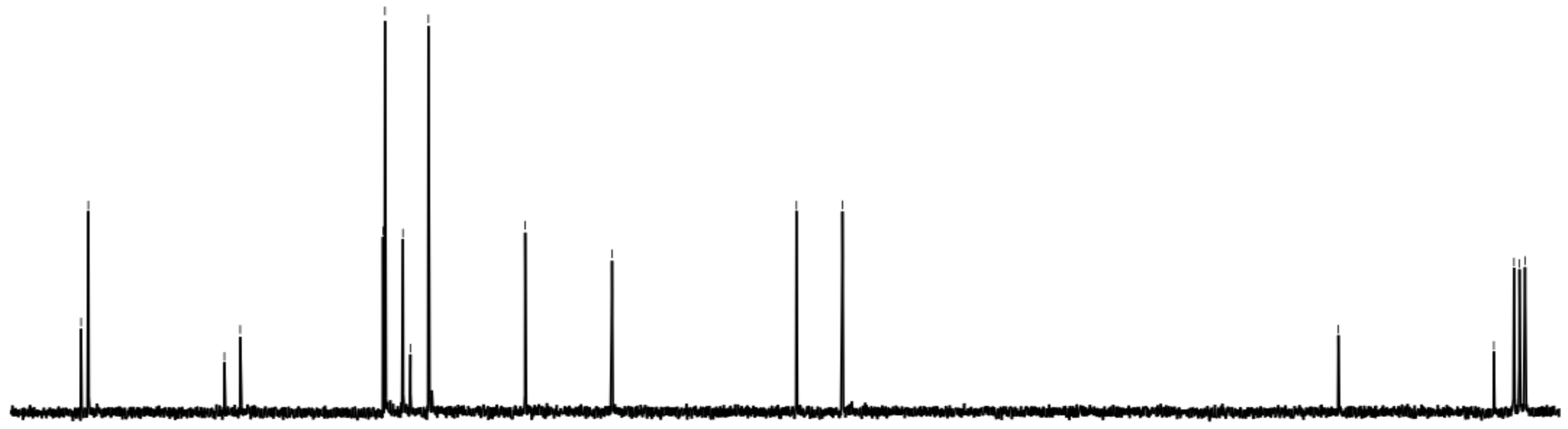




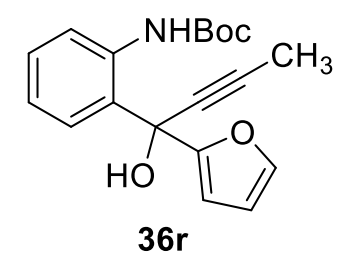

$500 \mathrm{MHz} \mathrm{CDCl}_{3}$

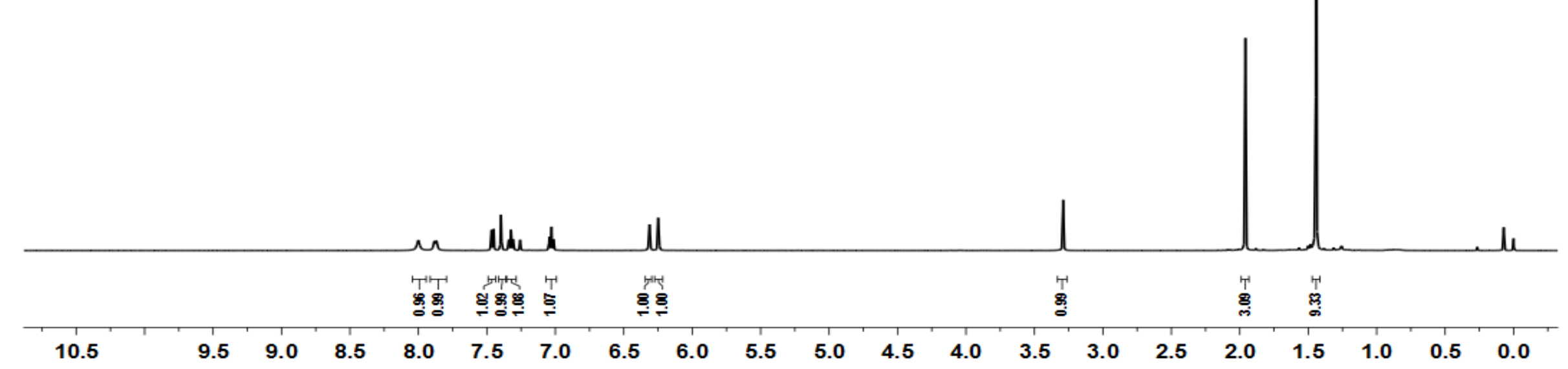




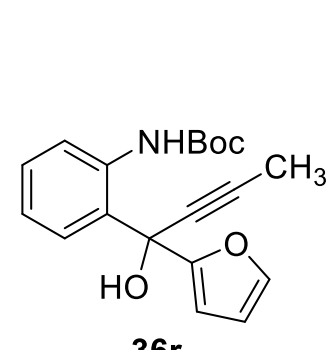

$100 \mathrm{MHz}, \mathrm{CDCl}_{3}$

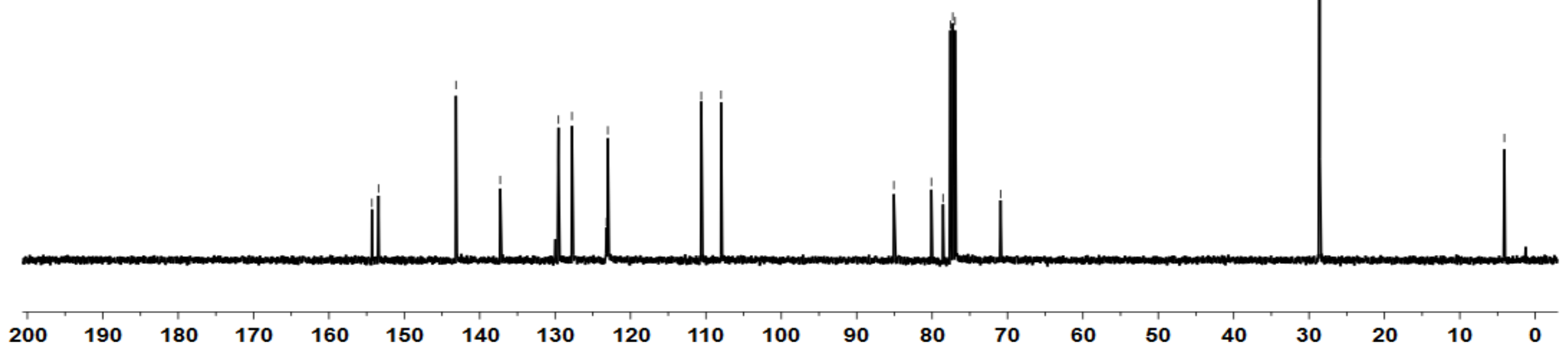




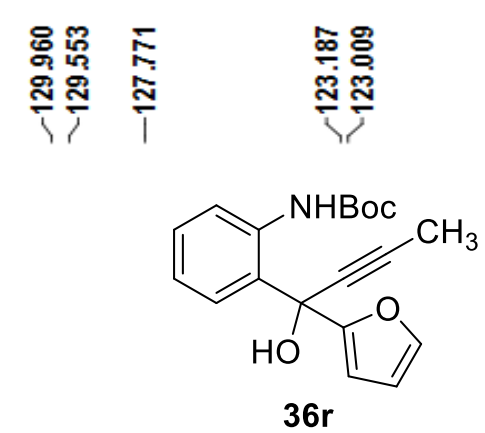

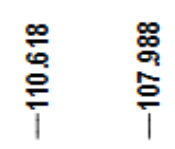

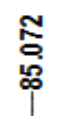

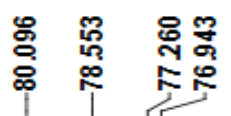

$100 \mathrm{MHz}, \mathrm{CDCl}_{3}$

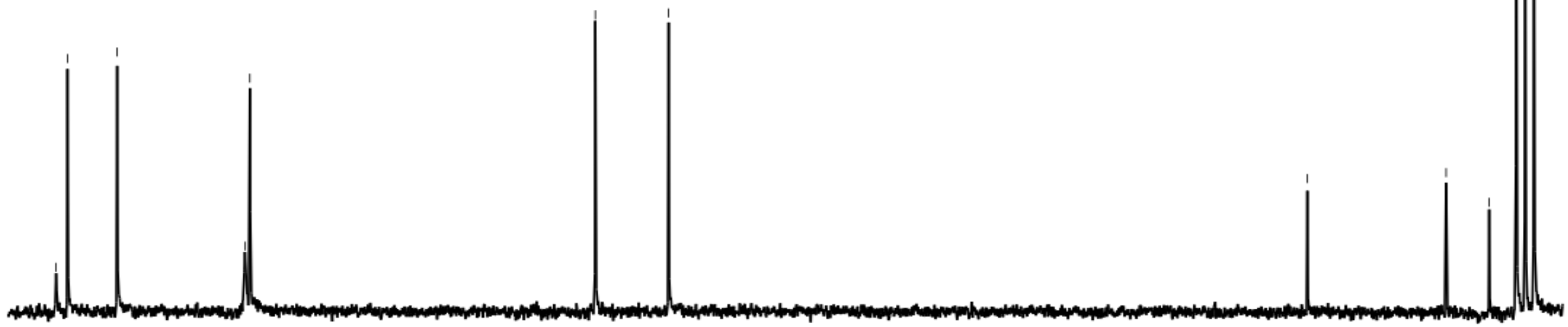




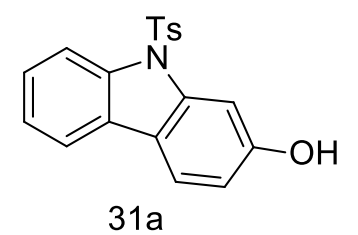

$400 \mathrm{MHz}, \mathrm{CDCl} 3$

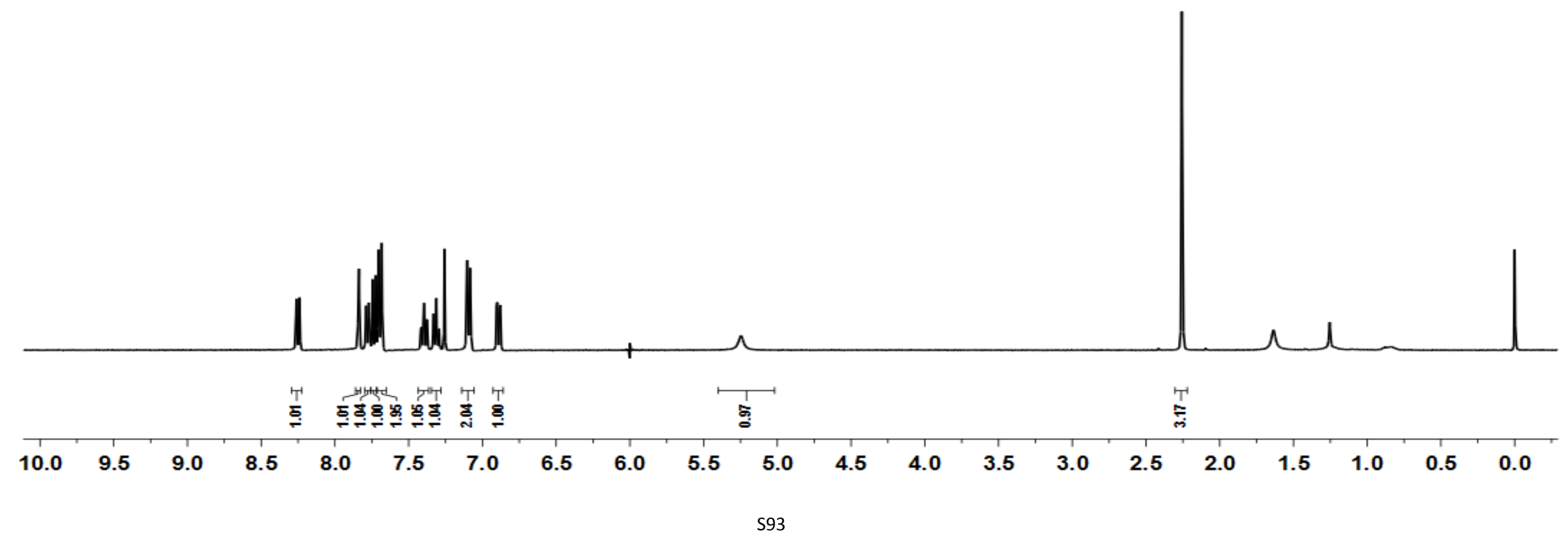




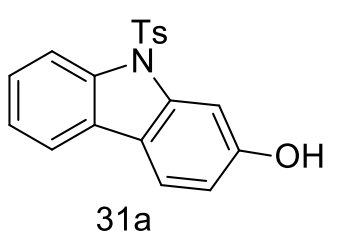

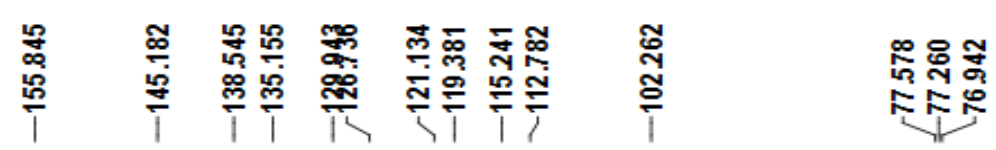

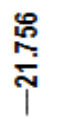

$100 \mathrm{MHz}, \mathrm{CDCl} 3$

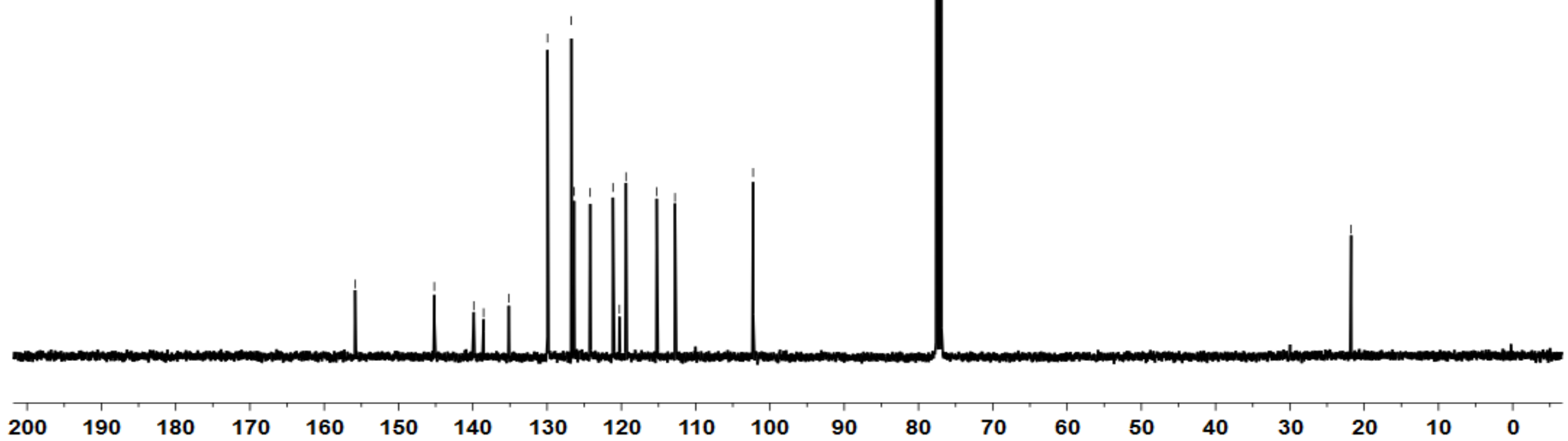



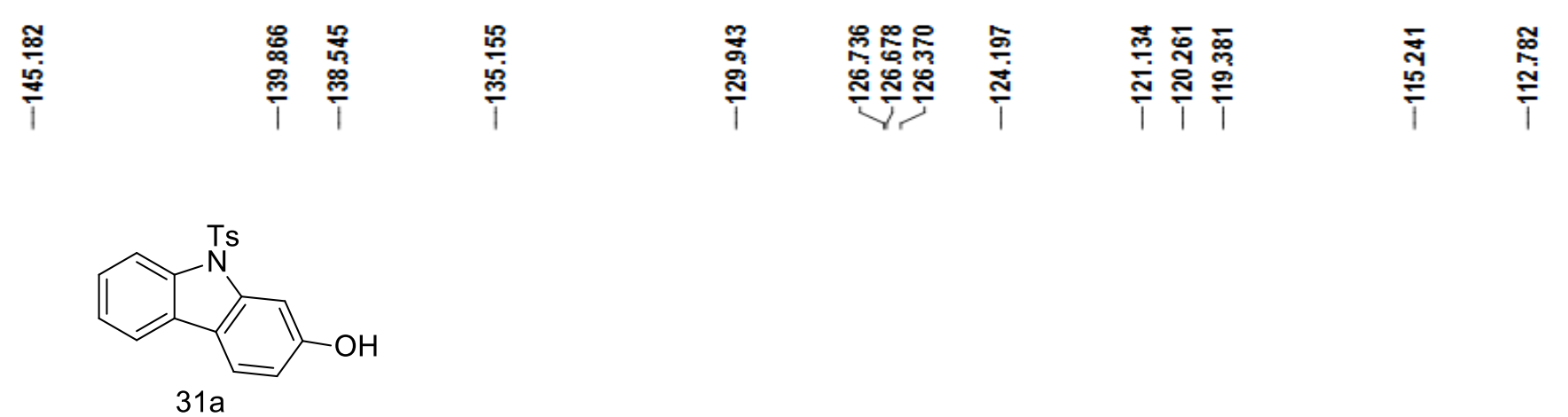

$100 \mathrm{MHz}, \mathrm{CDCl} 3$

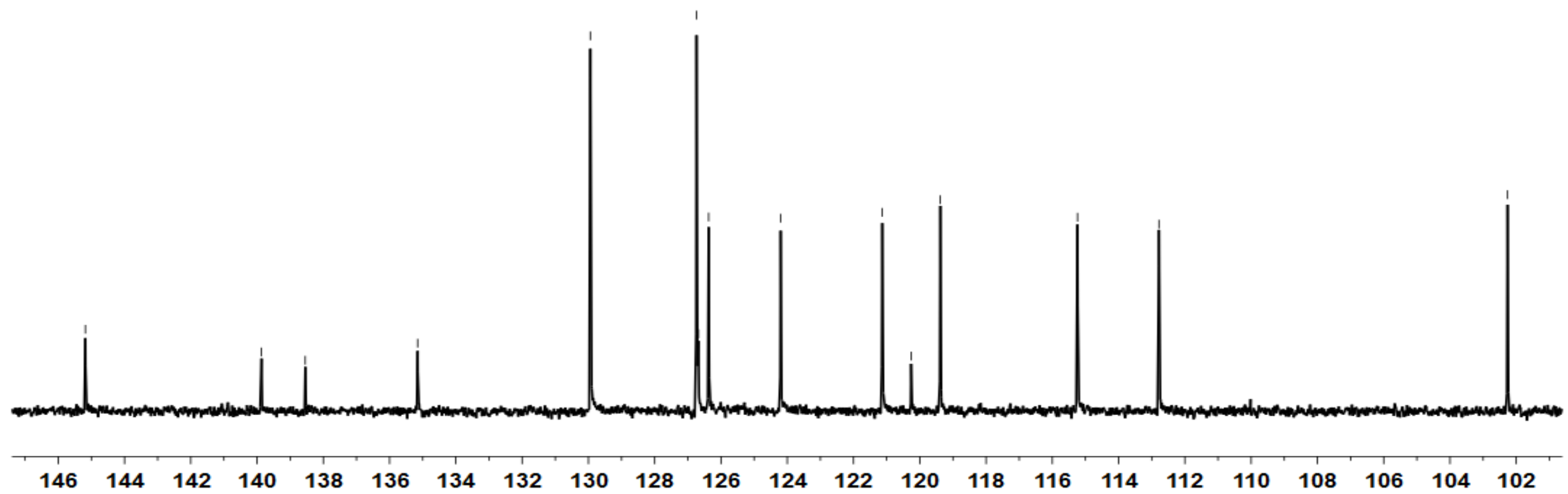




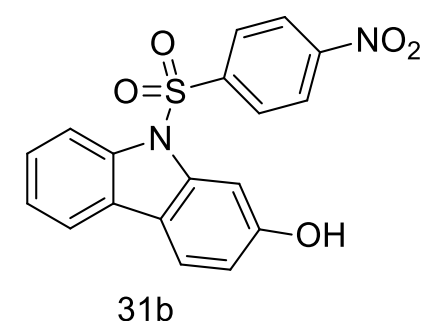

$500 \mathrm{MHz}, \mathrm{CD} 3 \mathrm{OD}$

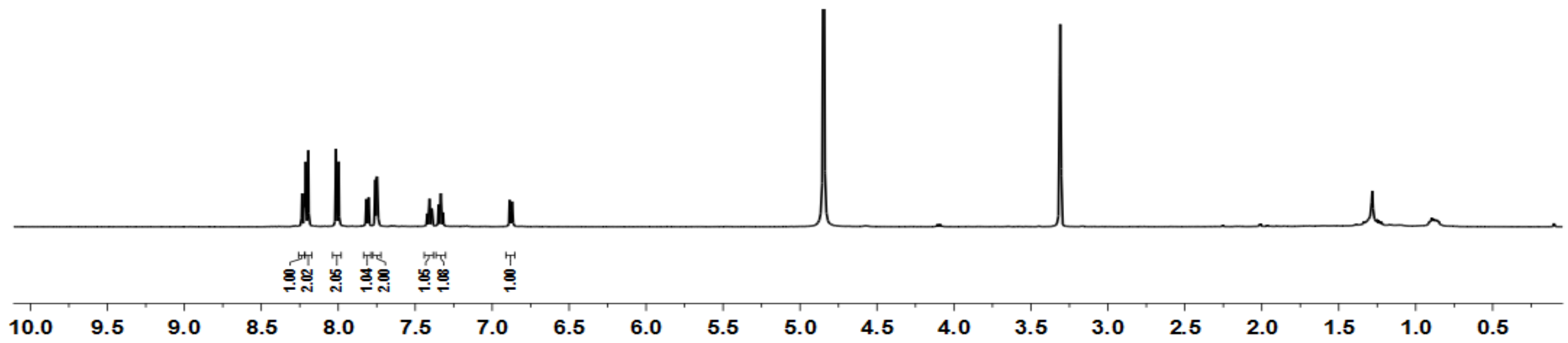




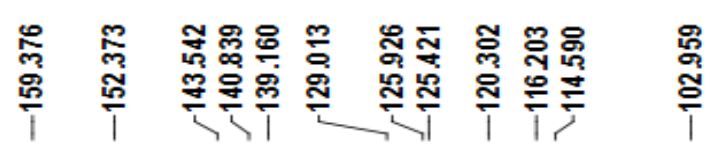

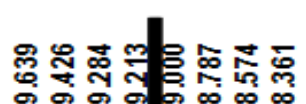

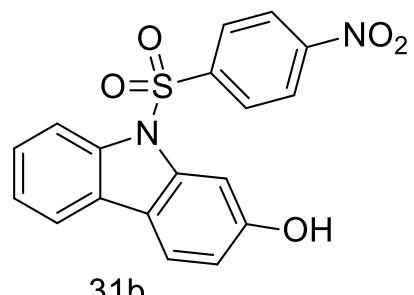

$100 \mathrm{MHz}, \mathrm{CD} 3 \mathrm{OD}$

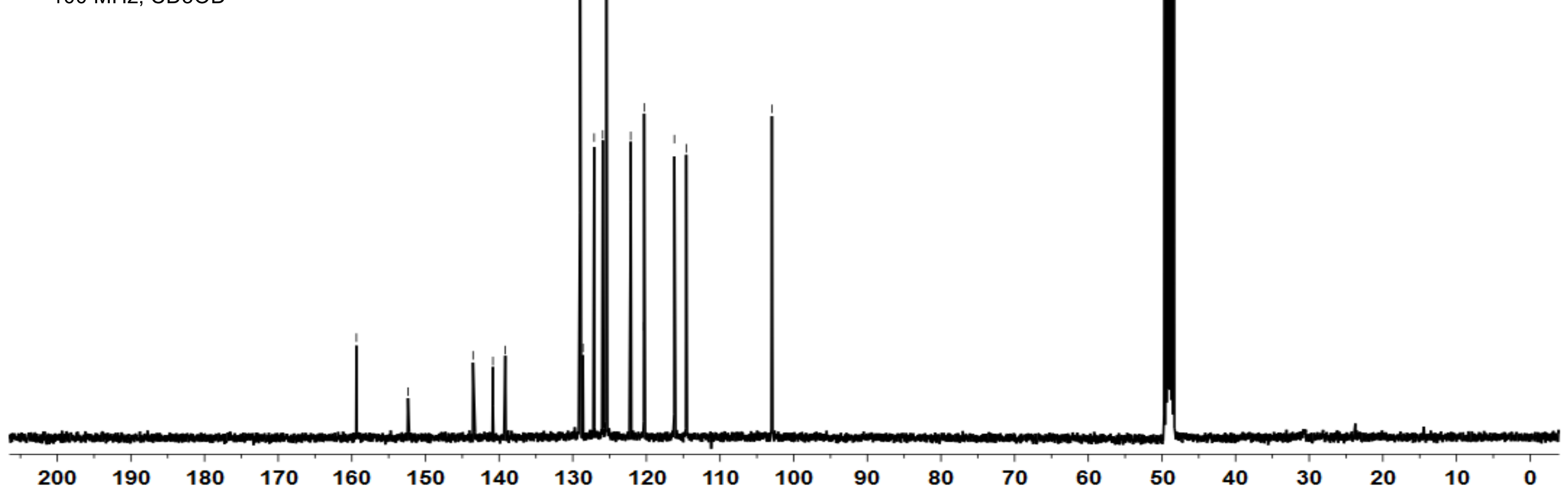




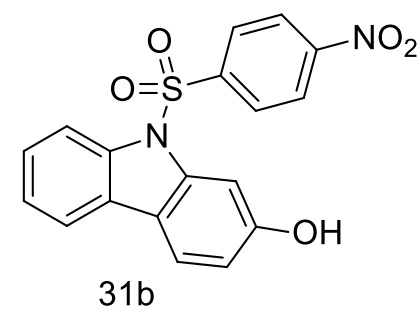

$100 \mathrm{MHz}, \mathrm{CD} 3 \mathrm{OD}$

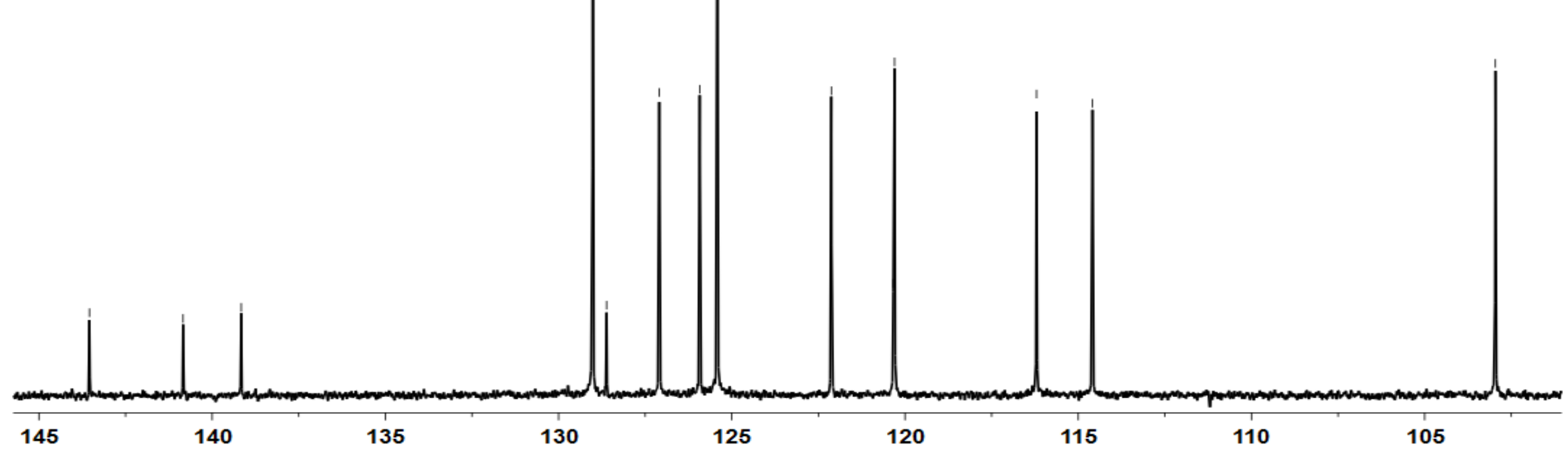




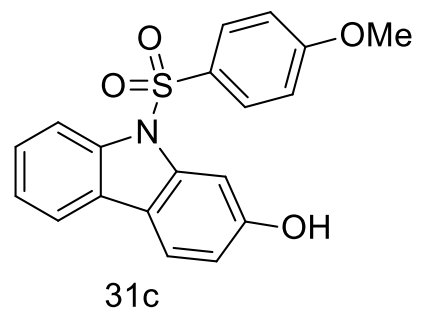

$500 \mathrm{MHz}, \mathrm{CD} 3 \mathrm{OD}$

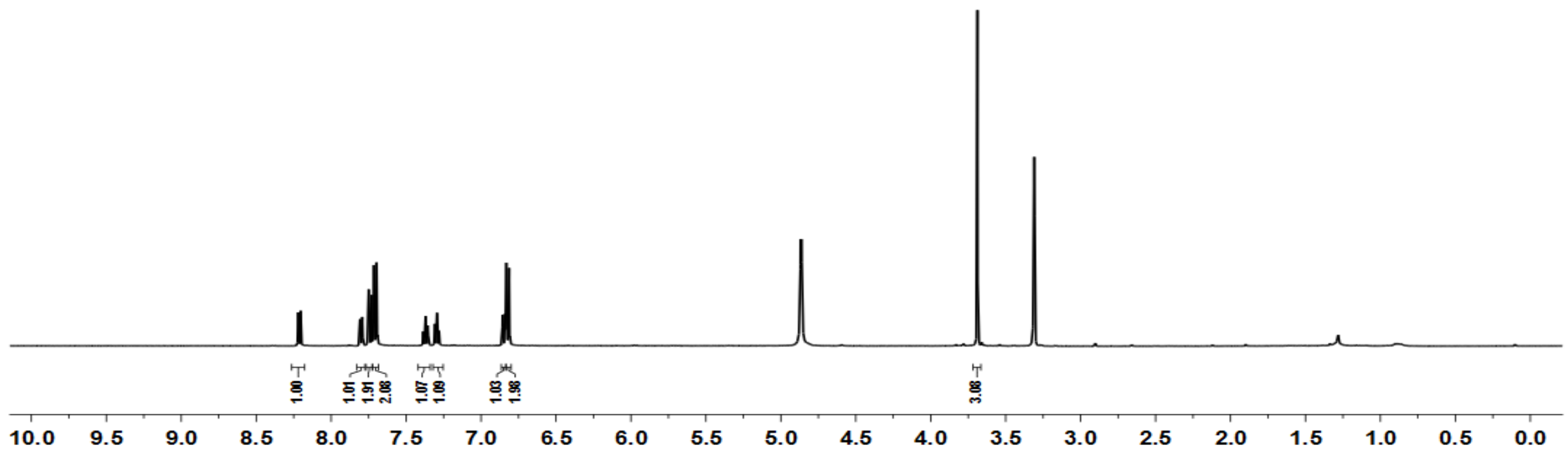




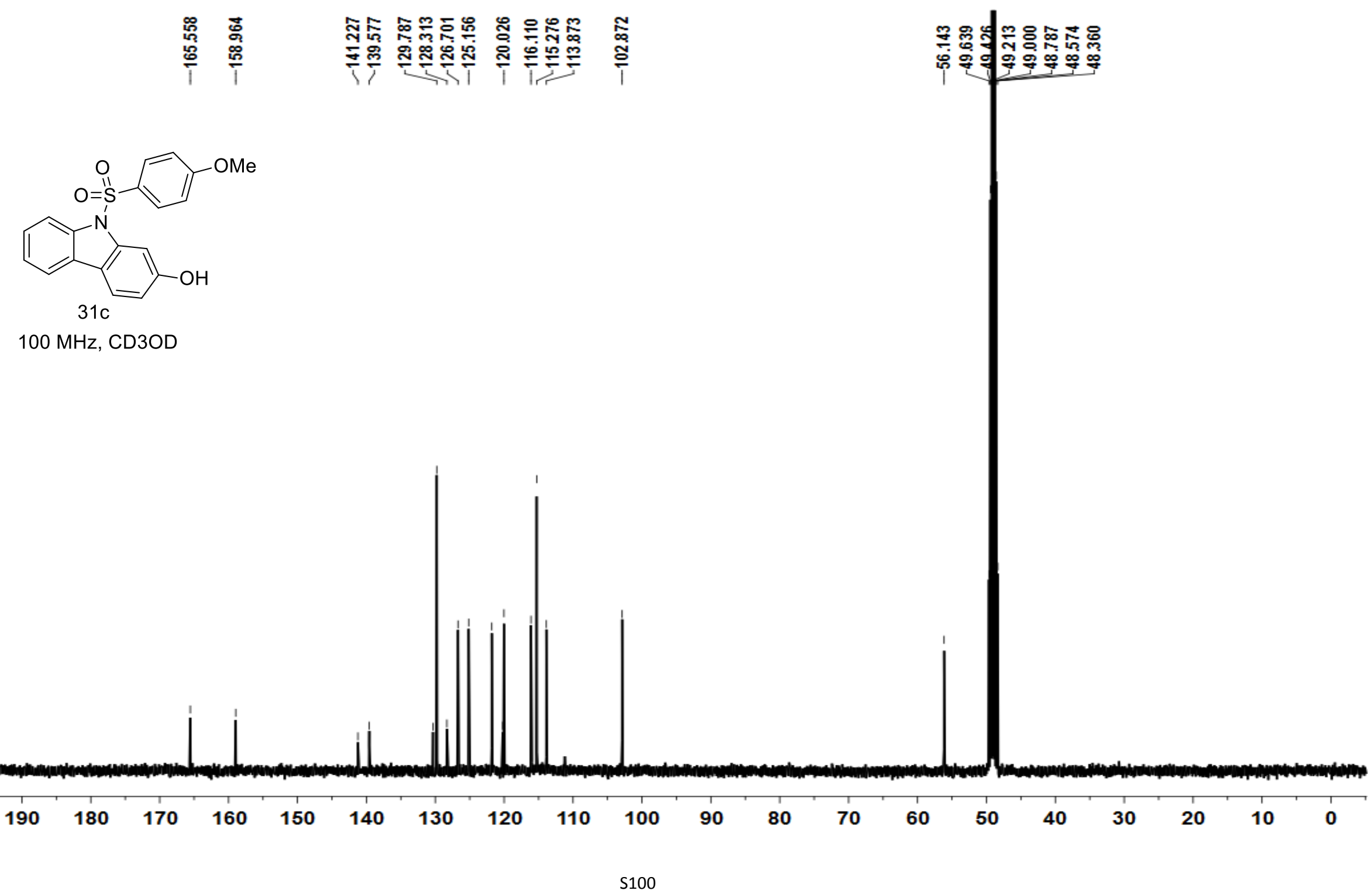




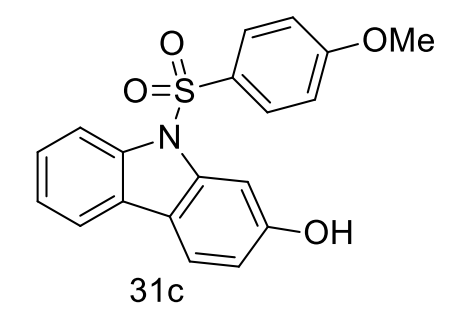

$100 \mathrm{MHz}, \mathrm{CD} 3 \mathrm{OD}$

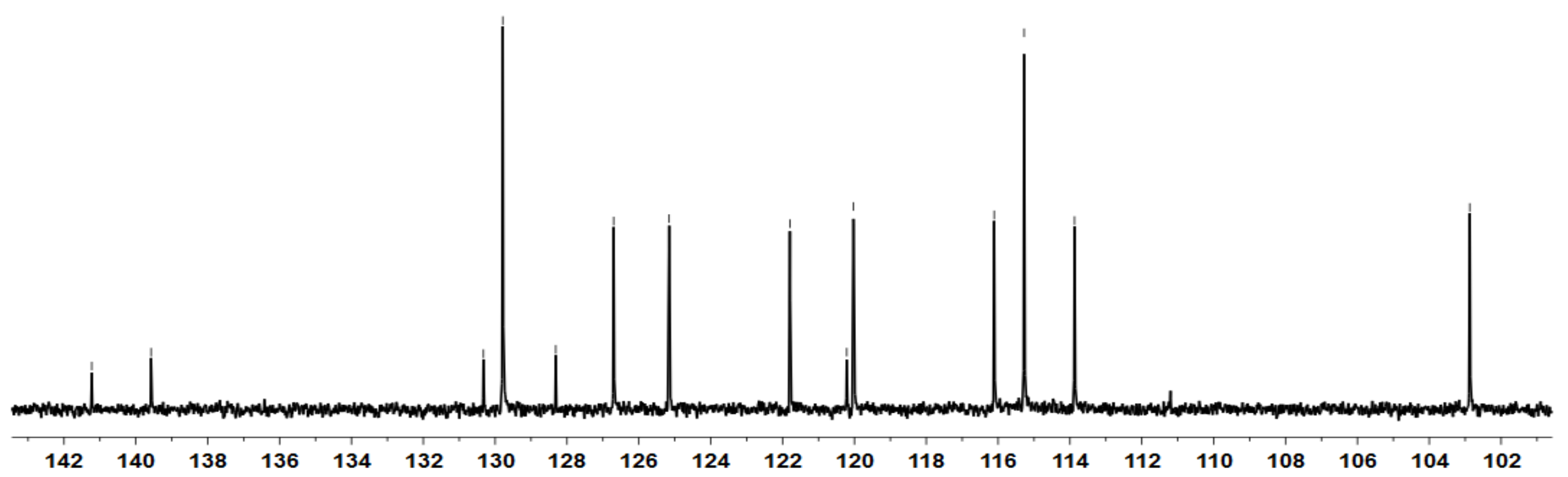




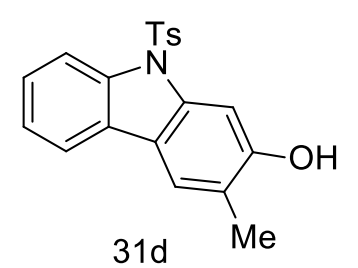

$500 \mathrm{MHz}, \mathrm{CDCl} 3$

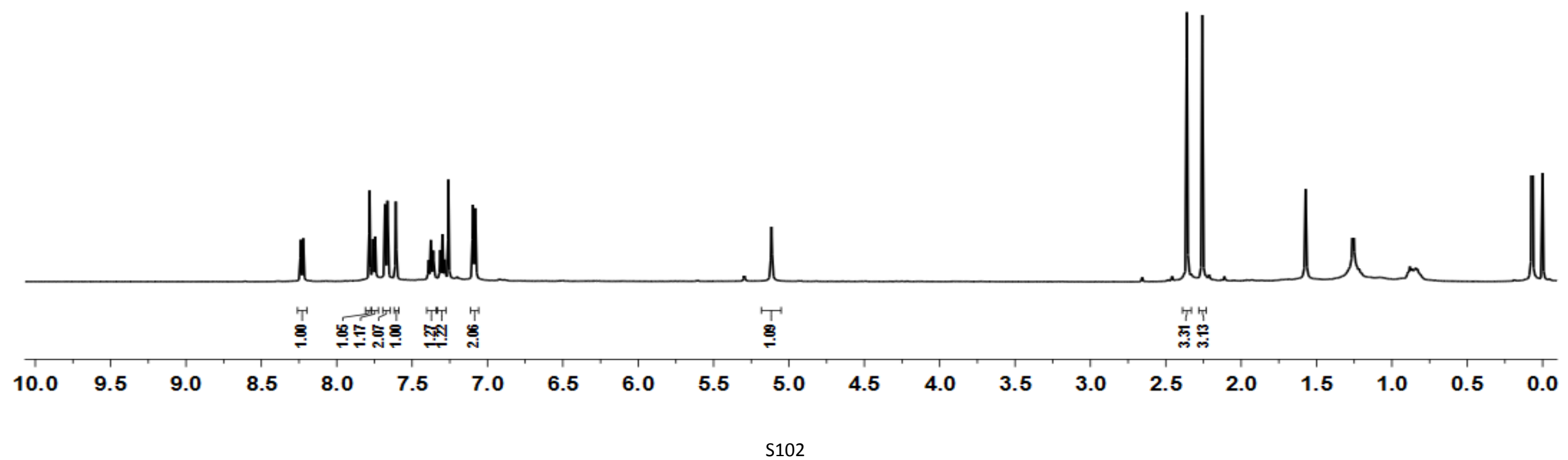



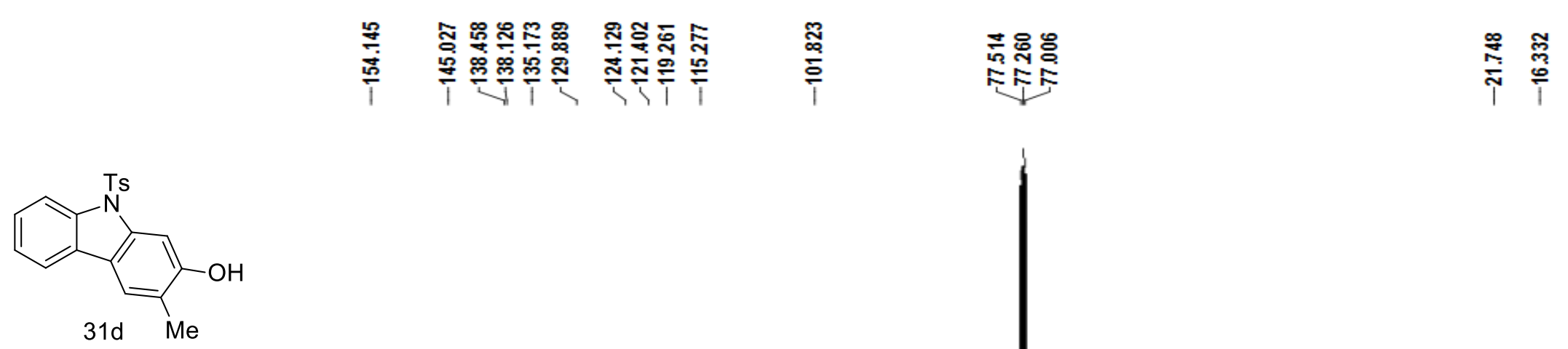

$125 \mathrm{MHz}, \mathrm{CDCl} 3$

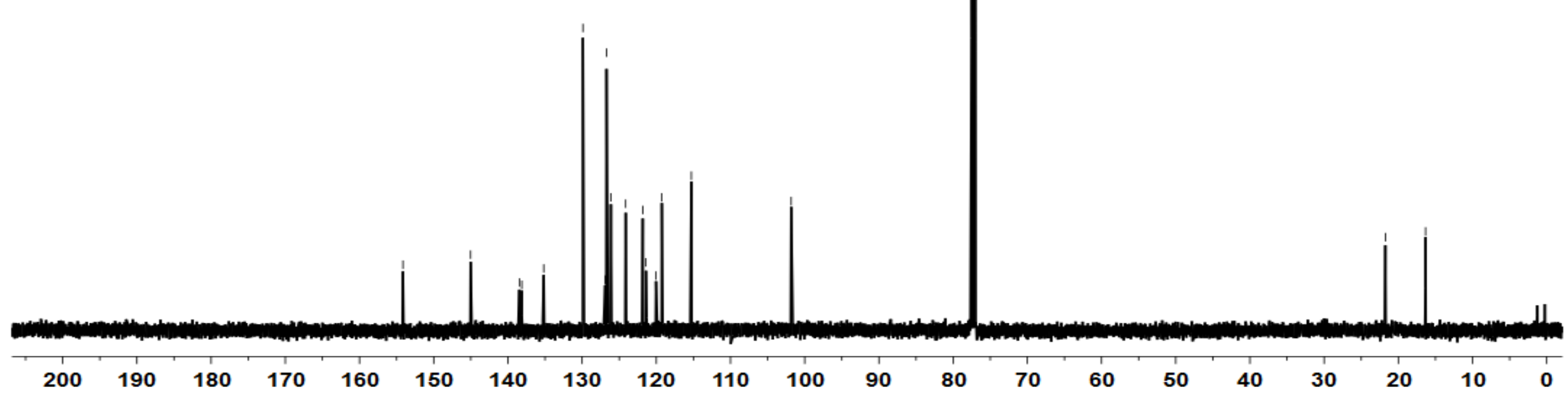




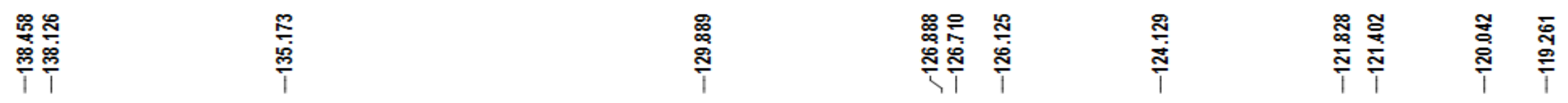

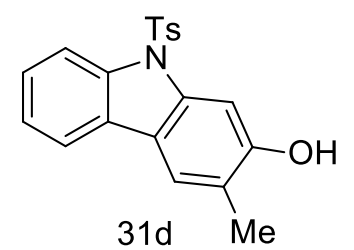

$125 \mathrm{MHz}, \mathrm{CDCl} 3$

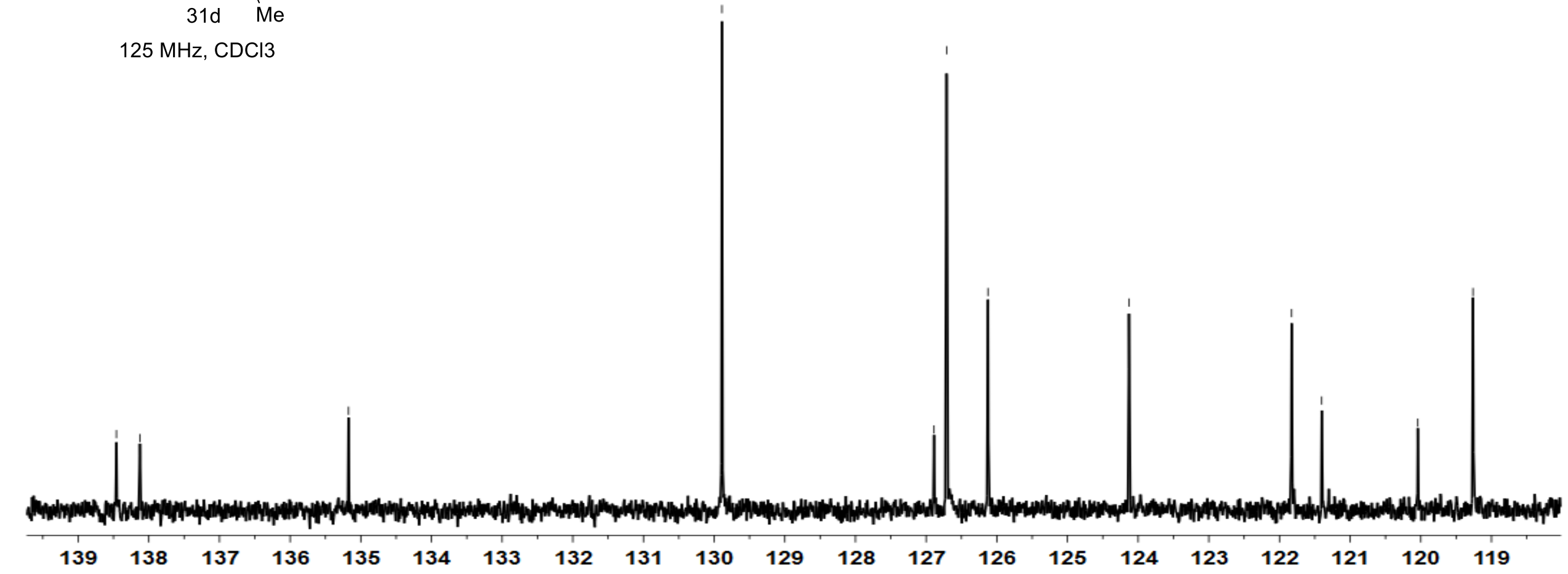




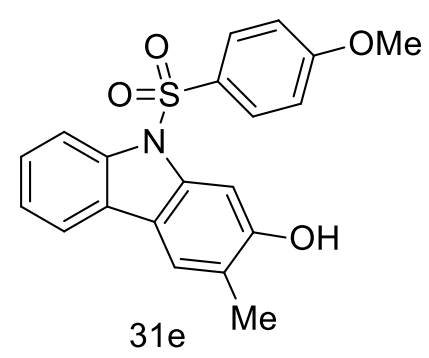

$500 \mathrm{MHz}, \mathrm{CDCl} 3$

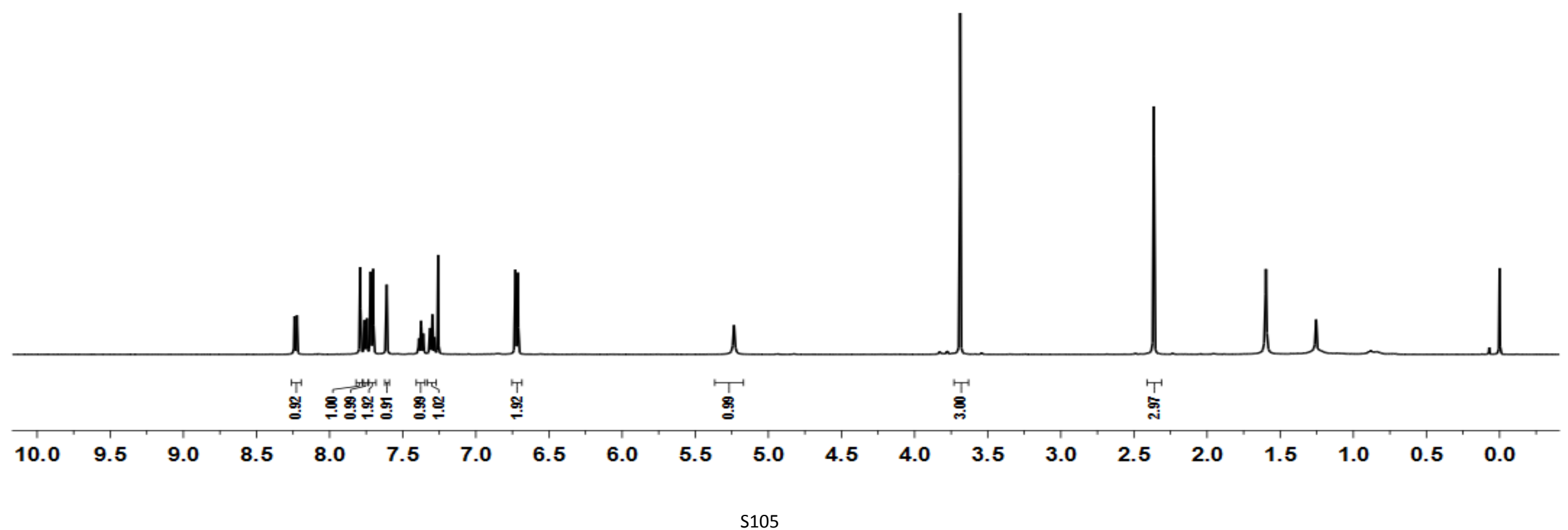




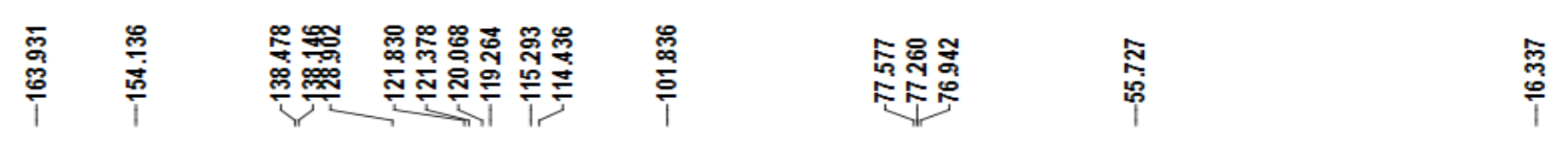

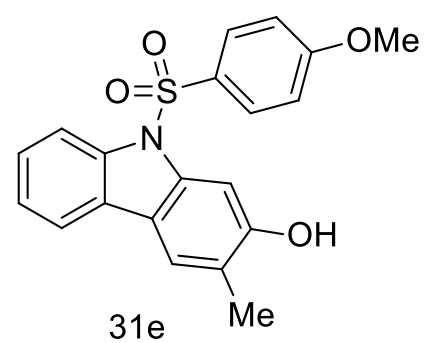

$100 \mathrm{MHz}, \mathrm{CDCl} 3$

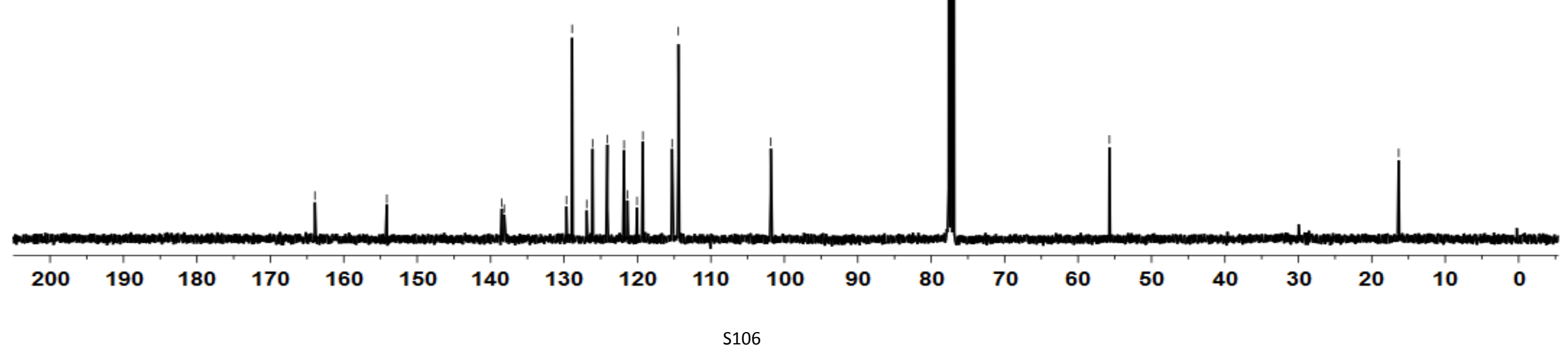




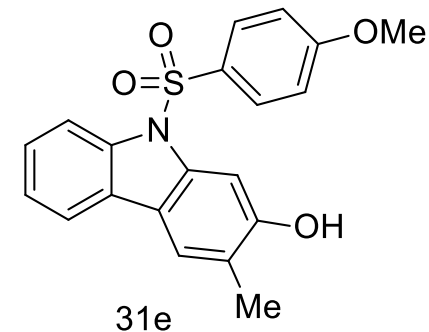

$100 \mathrm{MHz}, \mathrm{CDCl} 3$

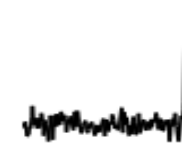

126

124

122 


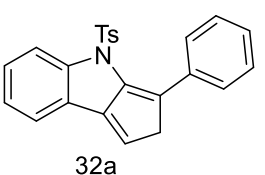

$500 \mathrm{MHz}, \mathrm{CDCl} 3$

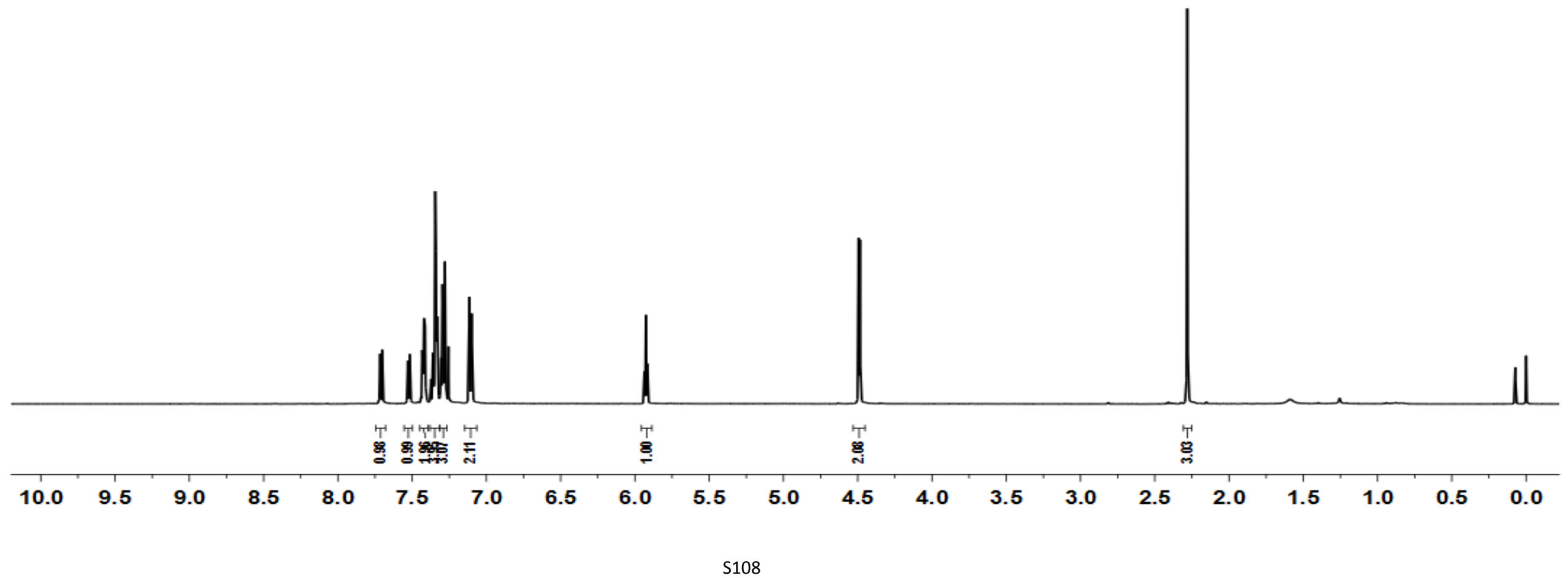




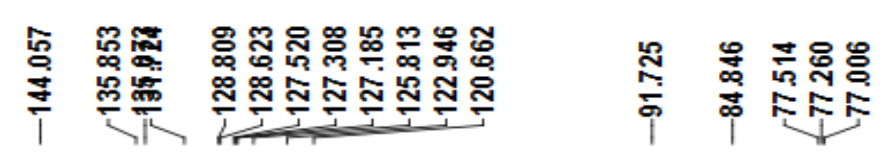

$\frac{1}{5}$

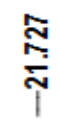

$\mathrm{MHz}, \mathrm{CDCl} 3$

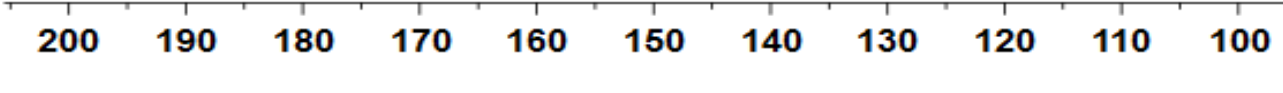




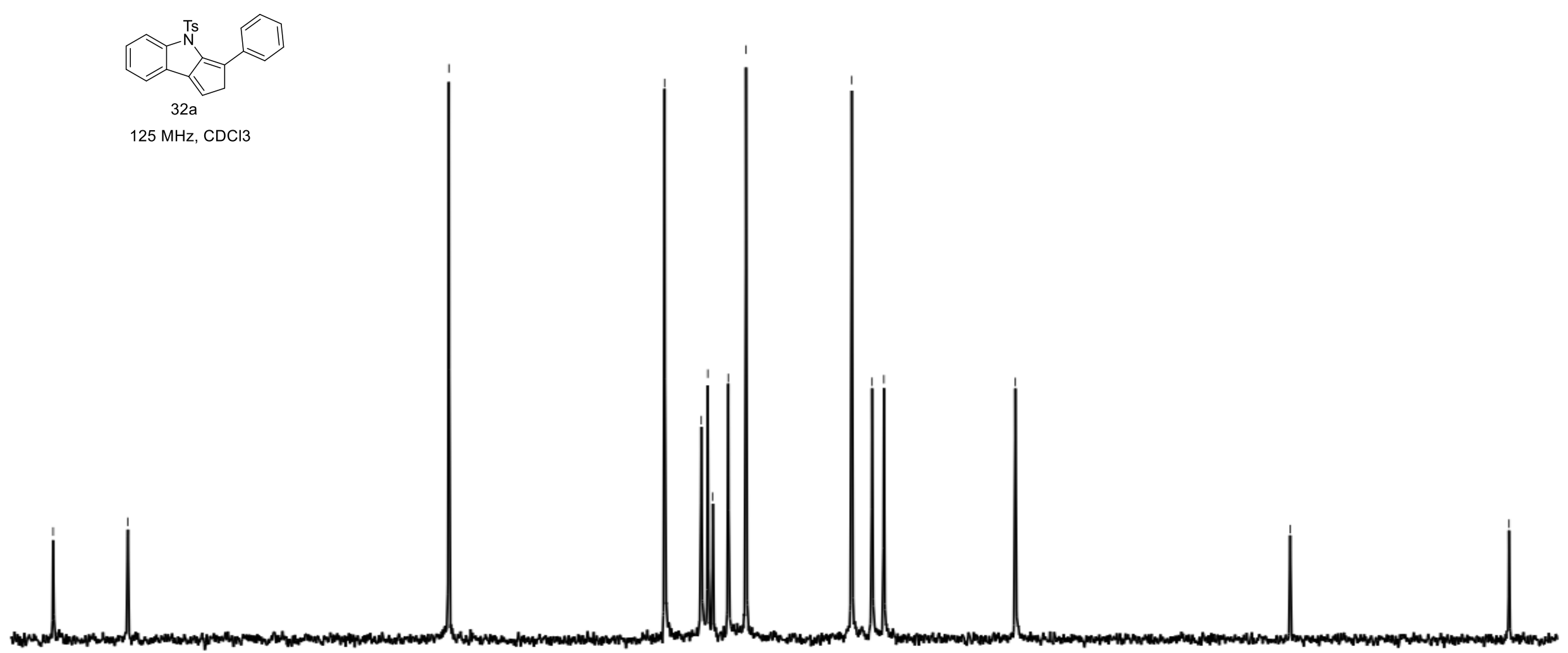




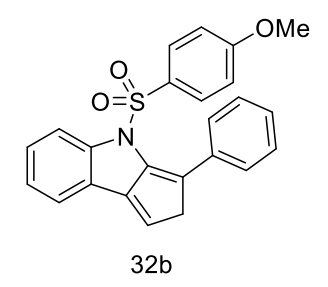

$400 \mathrm{MHz}, \mathrm{CDCl} 3$

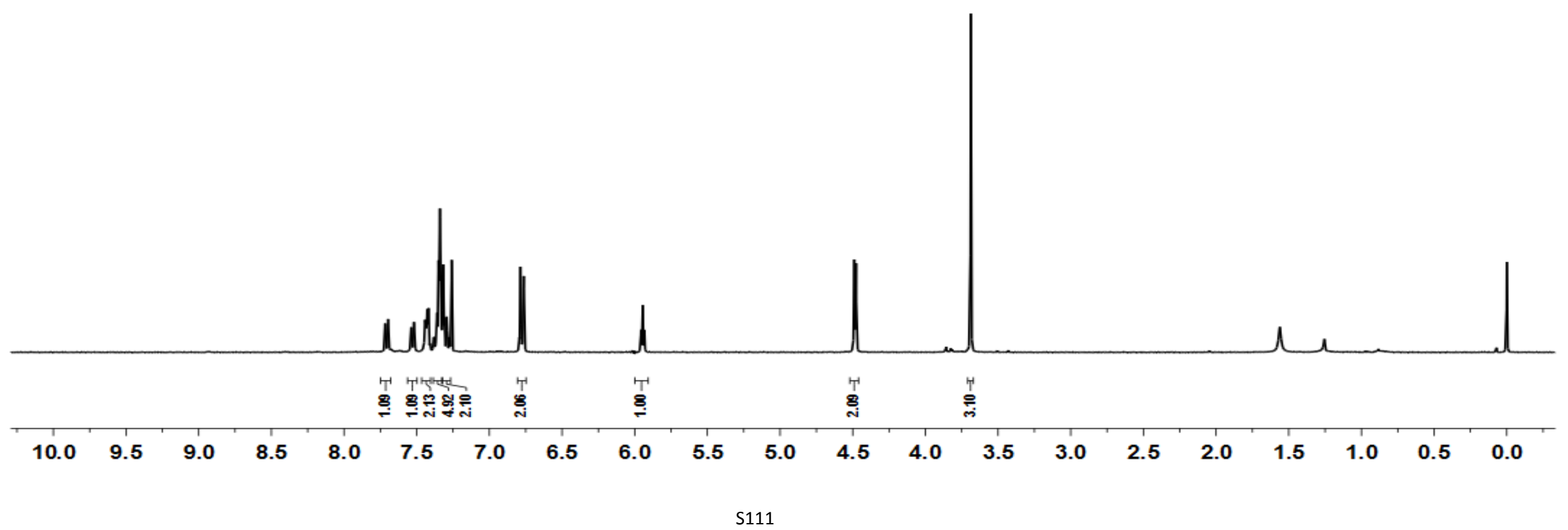




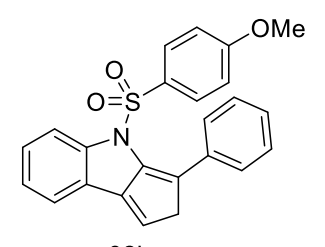

32

$100 \mathrm{MHz}, \mathrm{CDCl} 3$

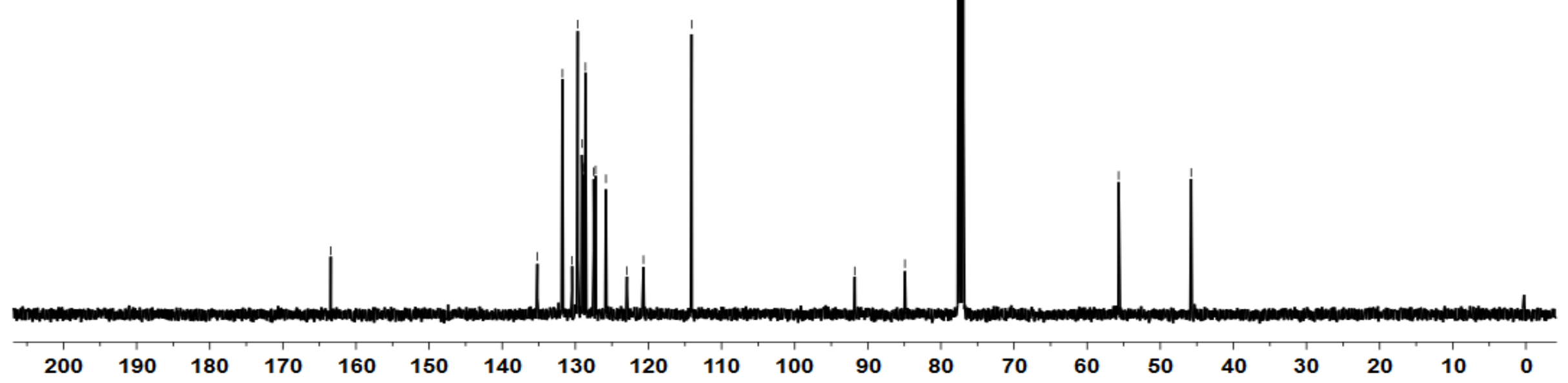




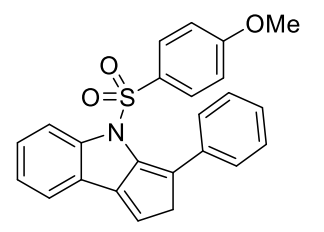

$32 \mathrm{~b}$

$100 \mathrm{MHz}, \mathrm{CDCl} 3$

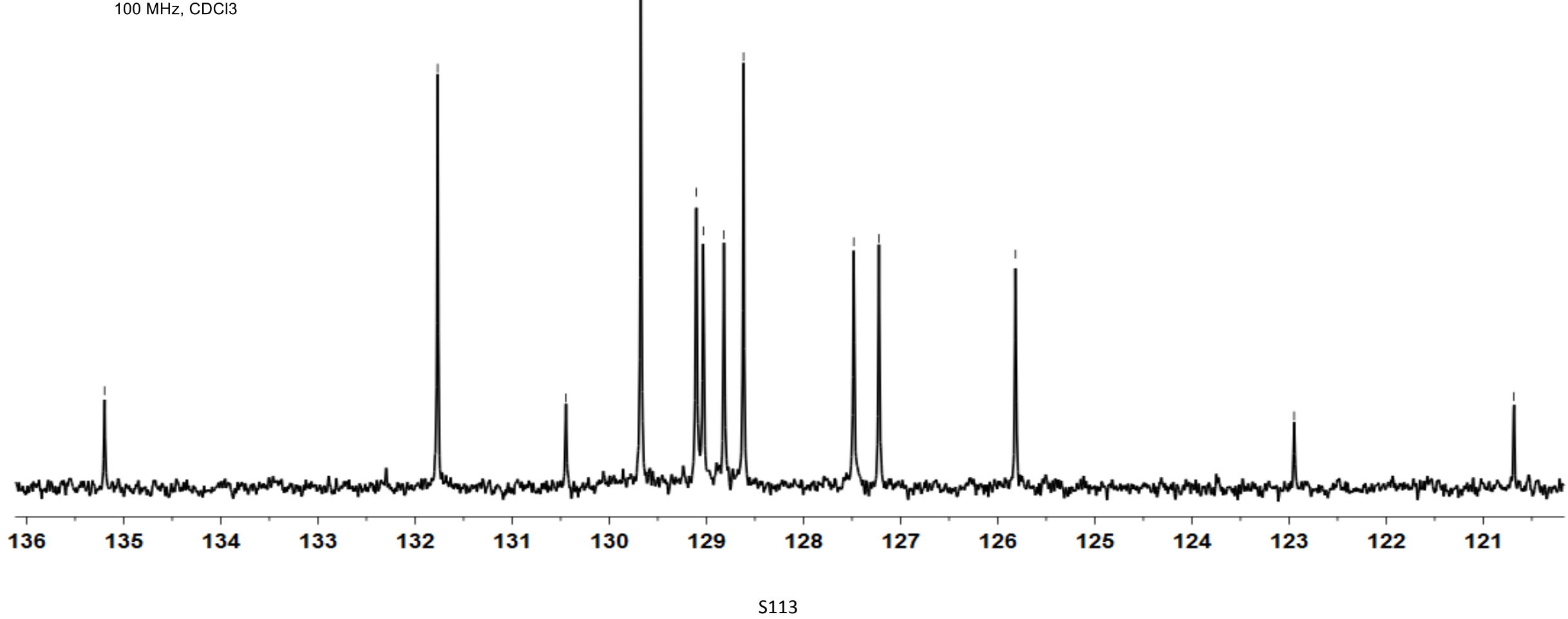




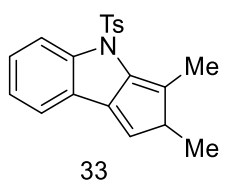

$400 \mathrm{MHz}, \mathrm{CDCl} 3$

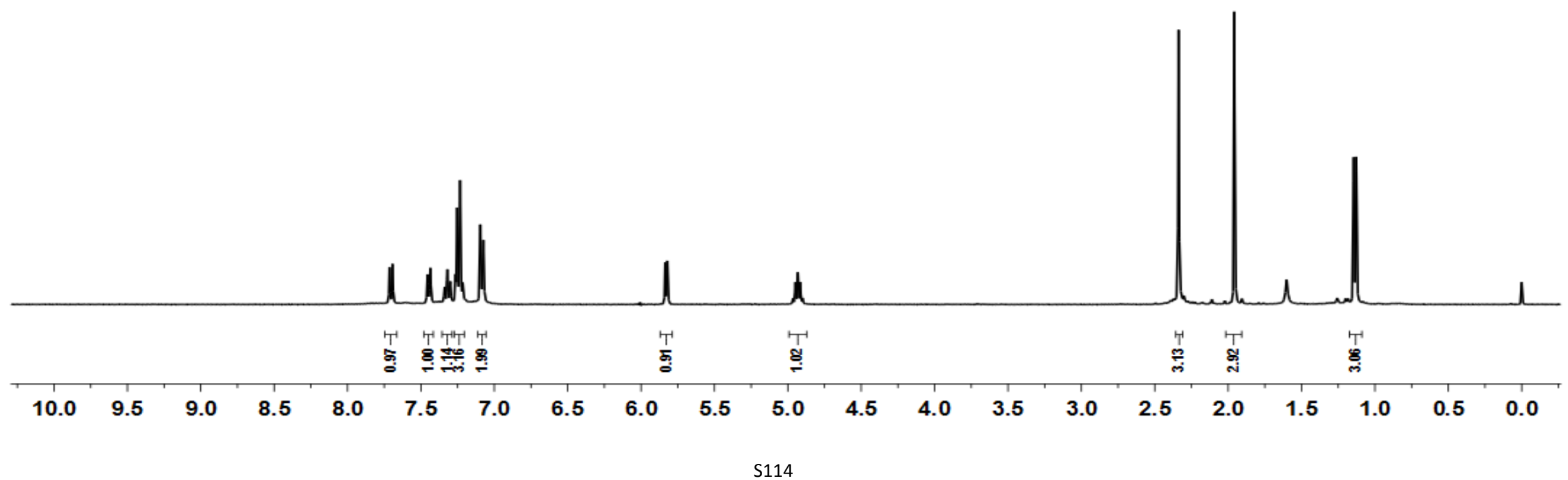



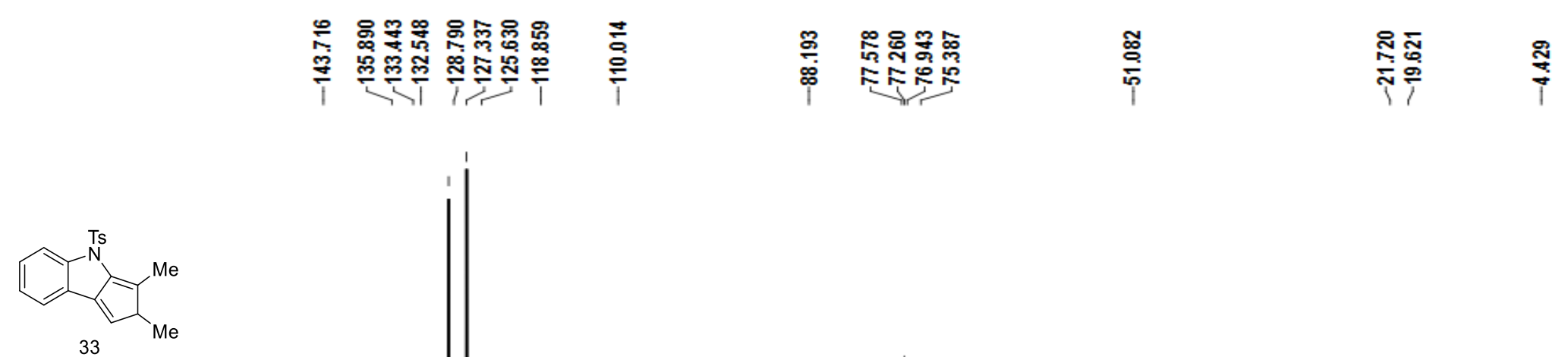

$100 \mathrm{MHz}, \mathrm{CDCl} 3$

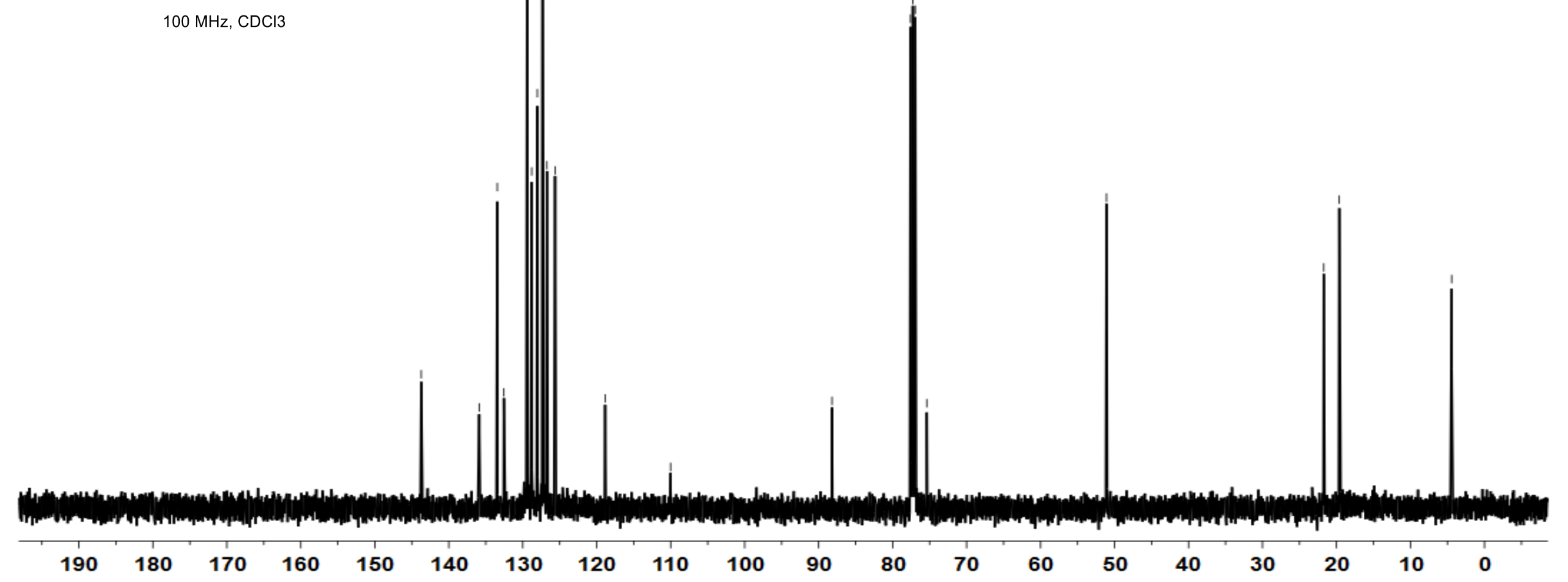




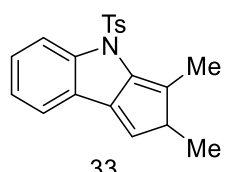

33

$100 \mathrm{MHz}, \mathrm{CDCl} 3$

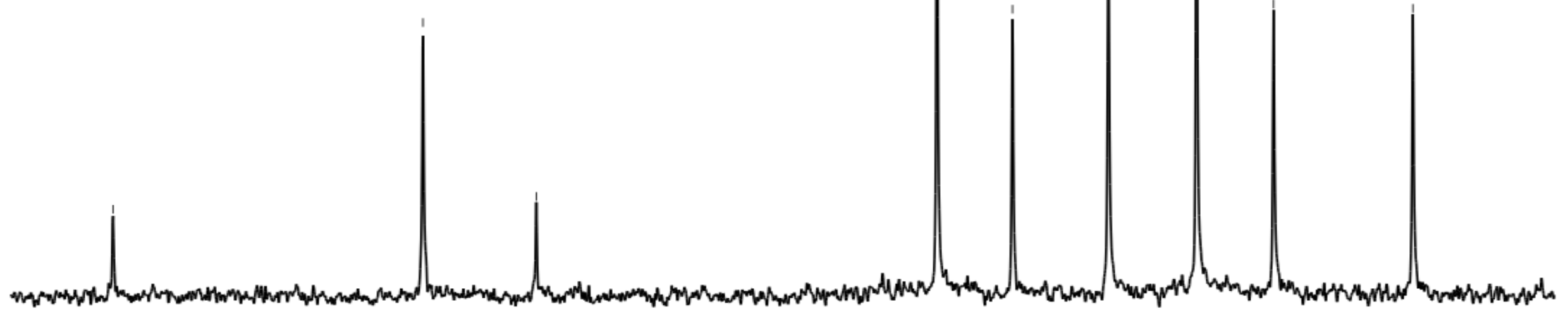




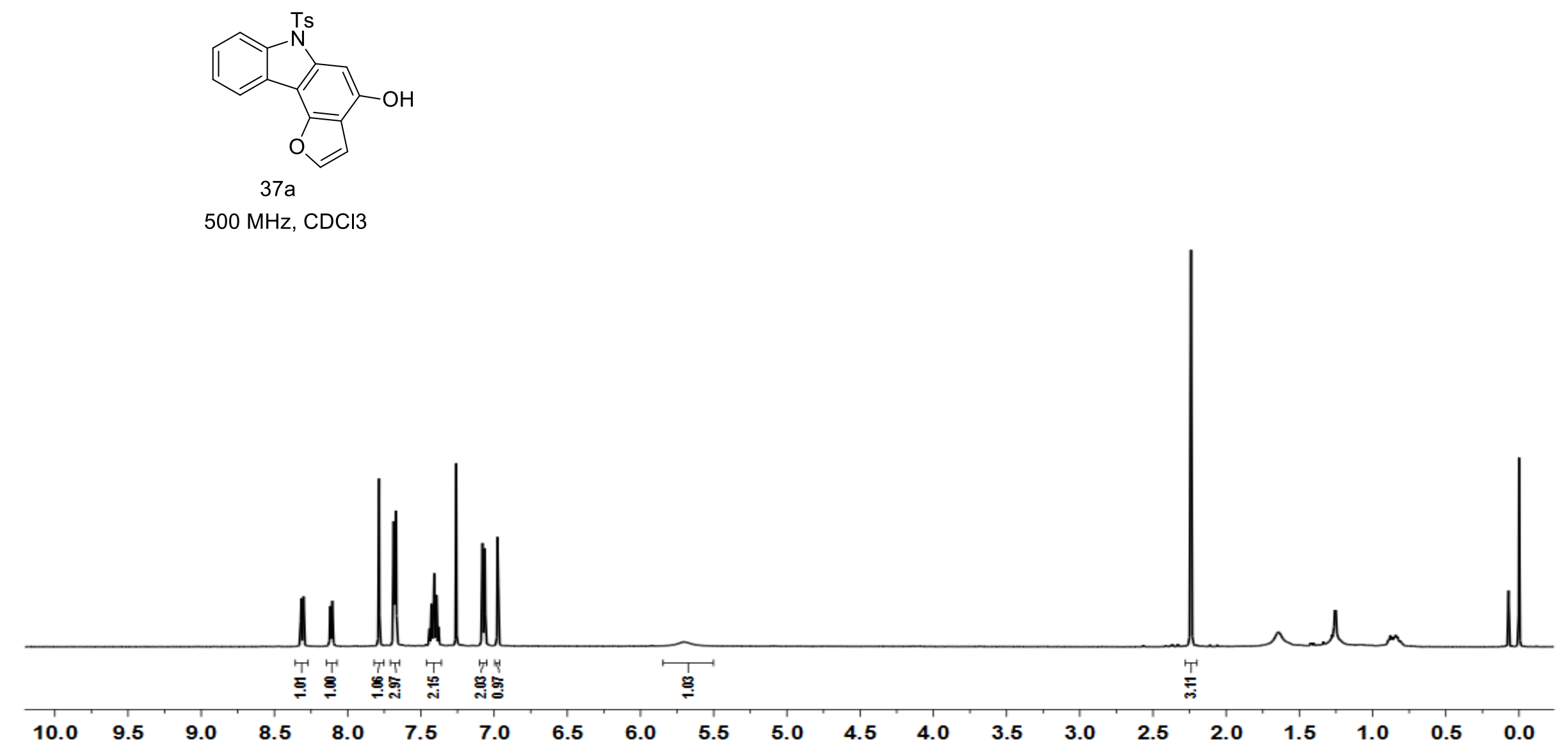




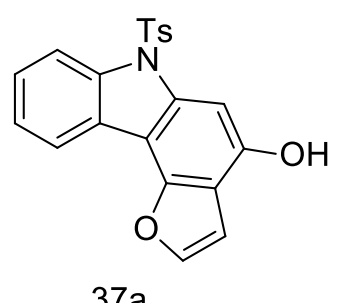

$37 a$

$125 \mathrm{MHz}, \mathrm{CDCl} 3$

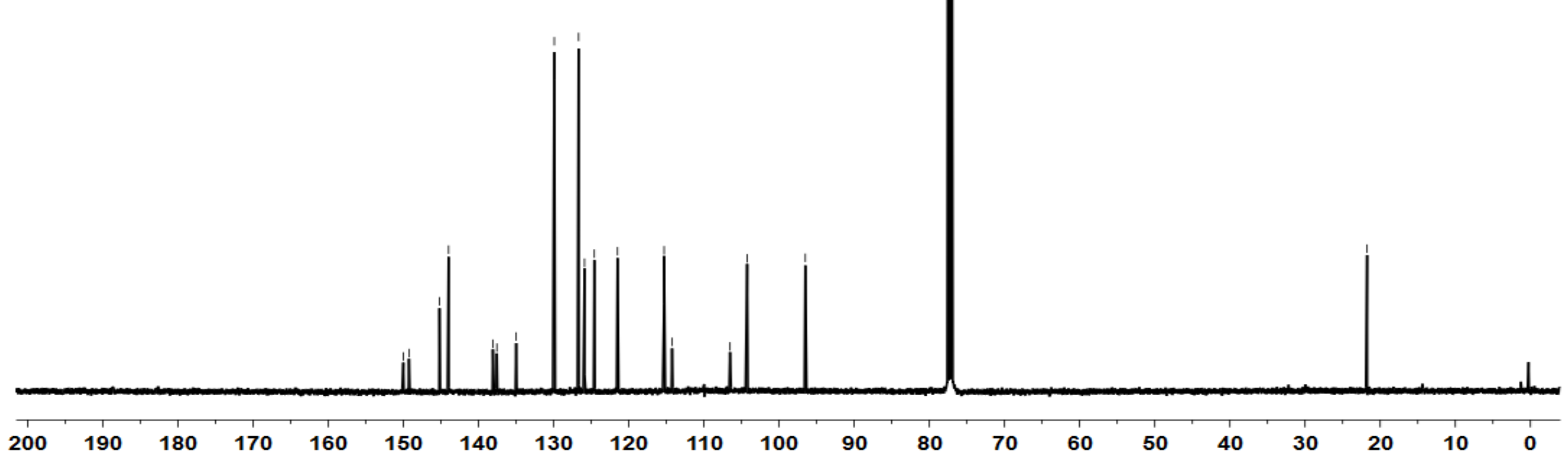




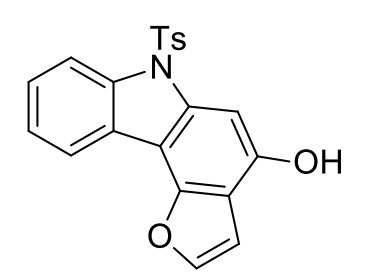

$37 a$

$125 \mathrm{MHz}, \mathrm{CDCl} 3$

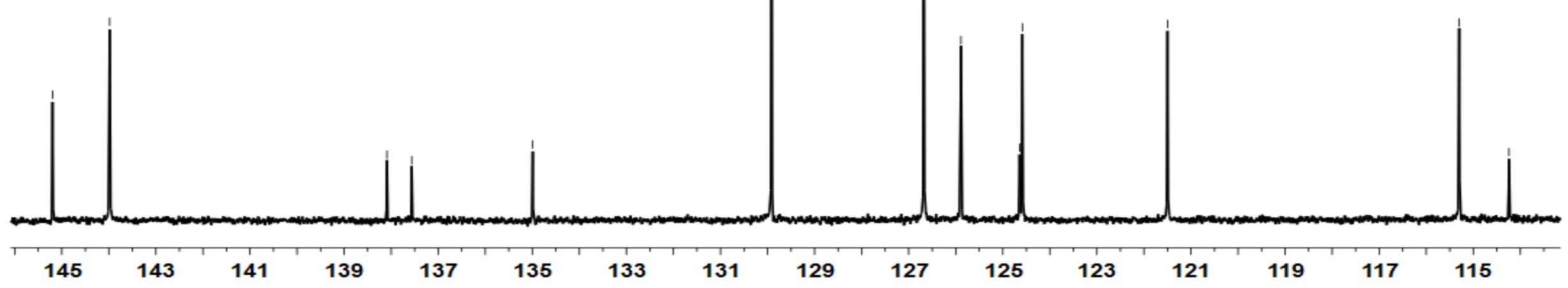




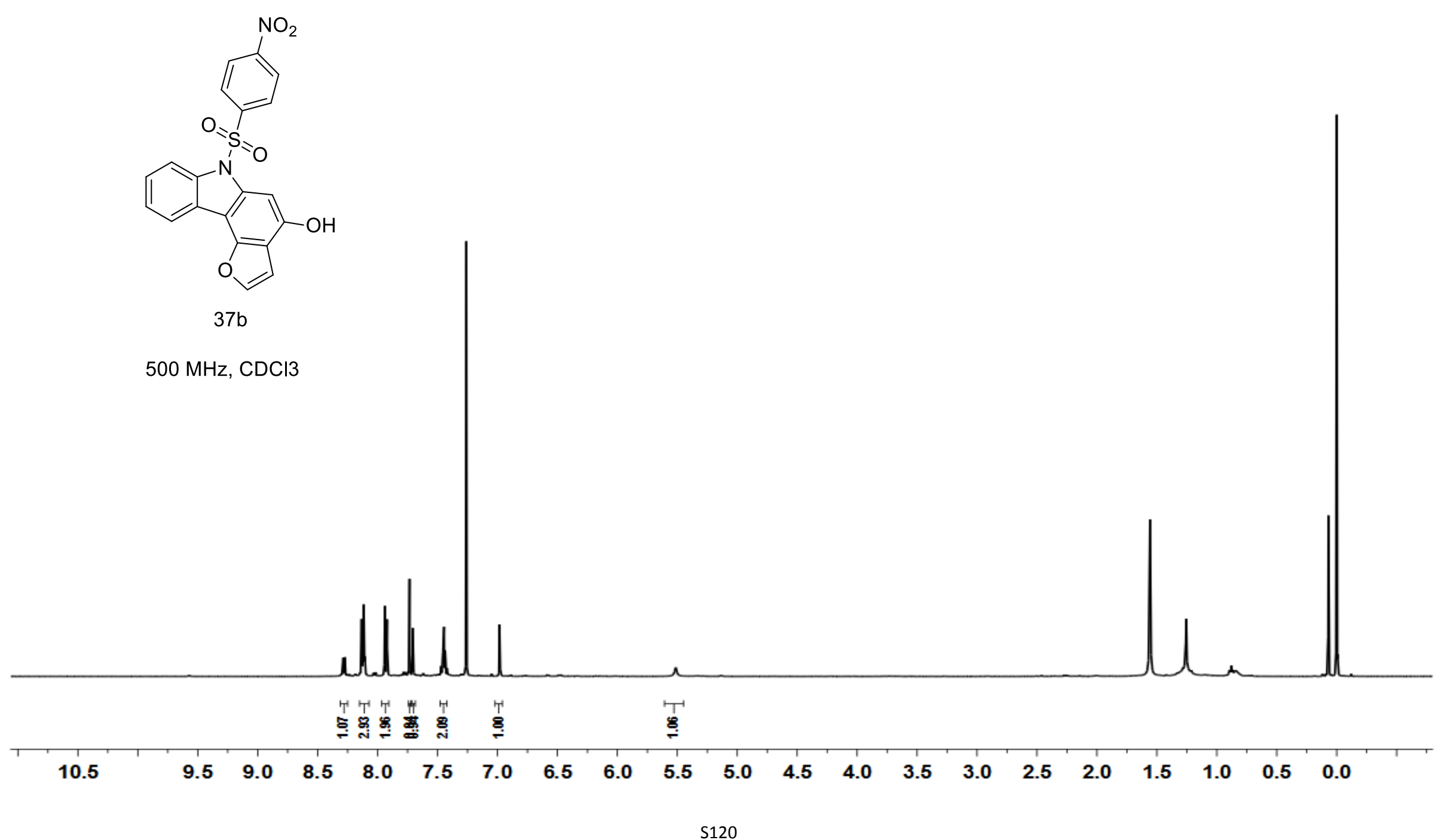




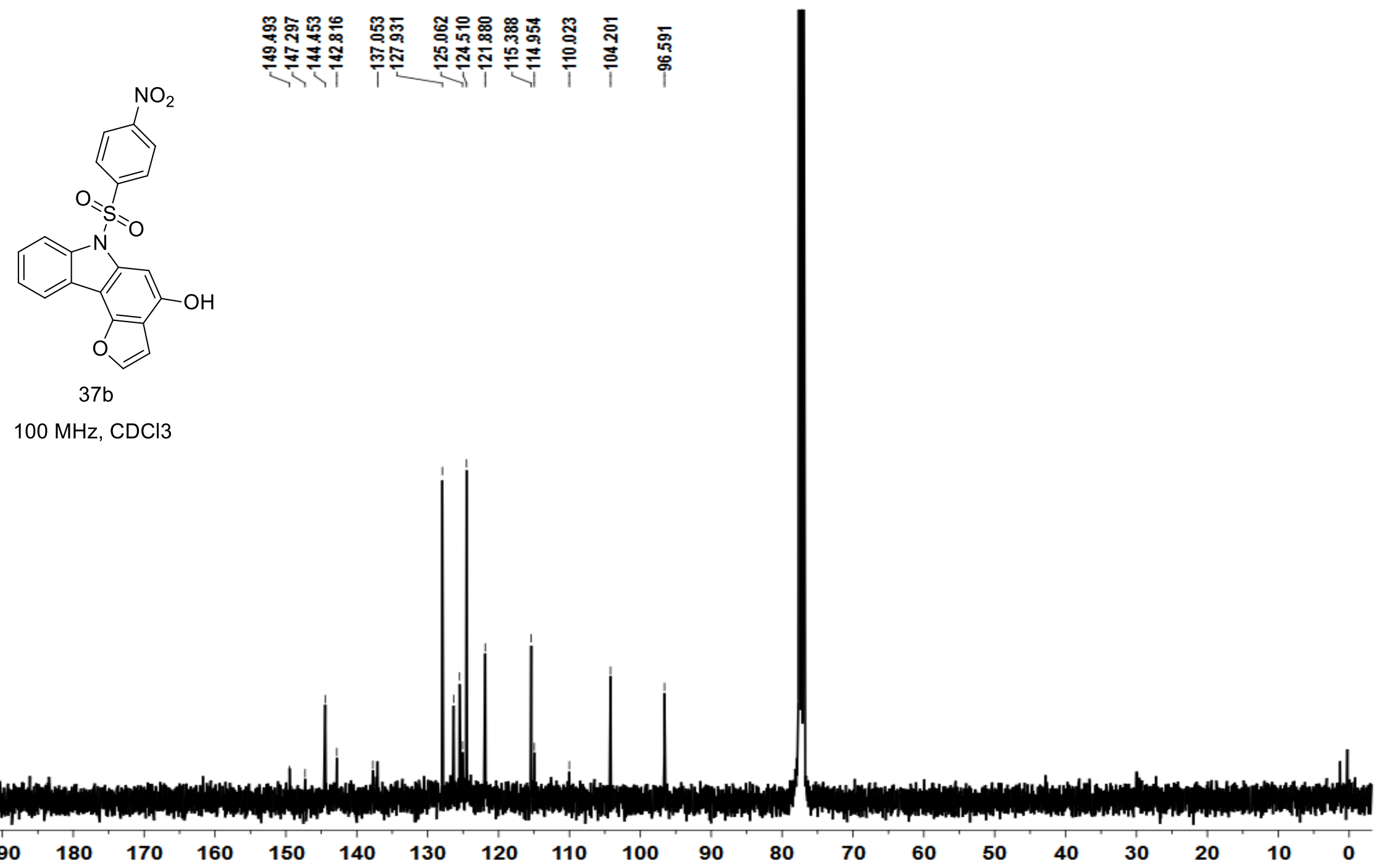




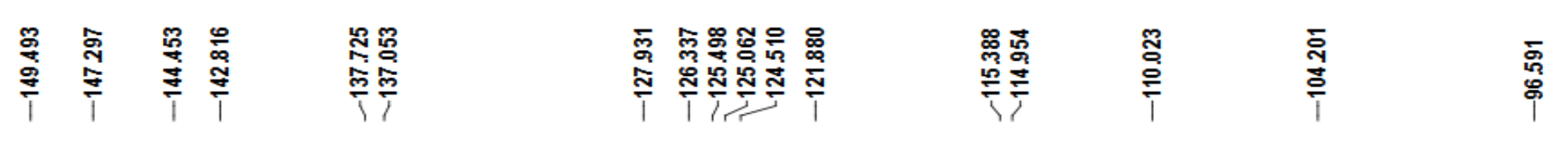

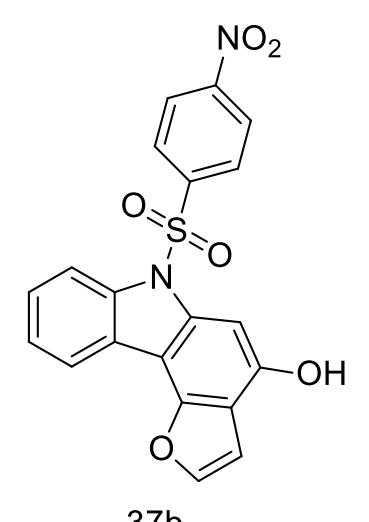

$100 \mathrm{MHz}, \mathrm{CDCl} 3$

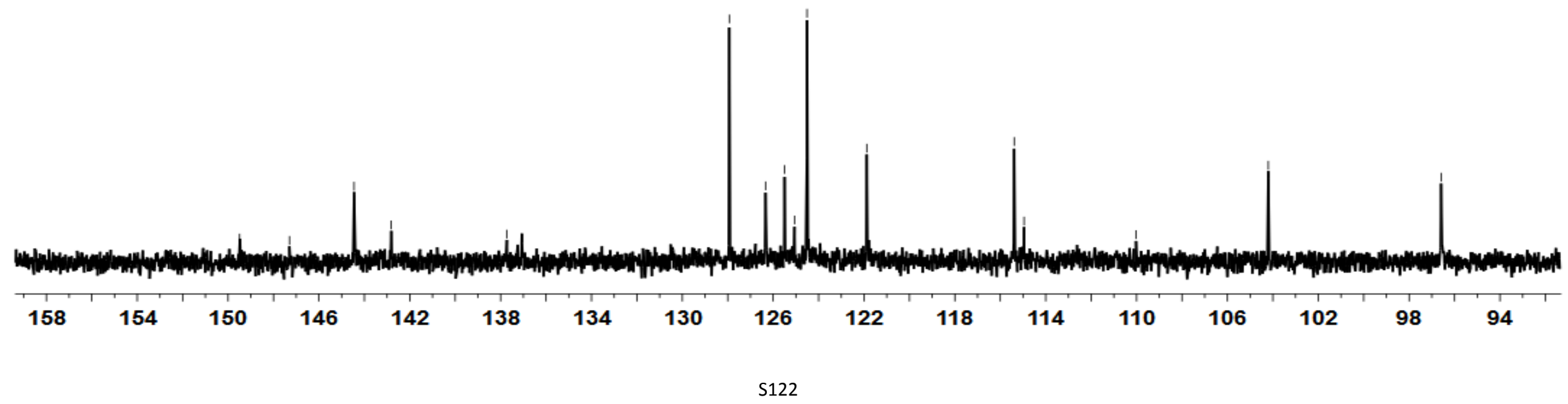




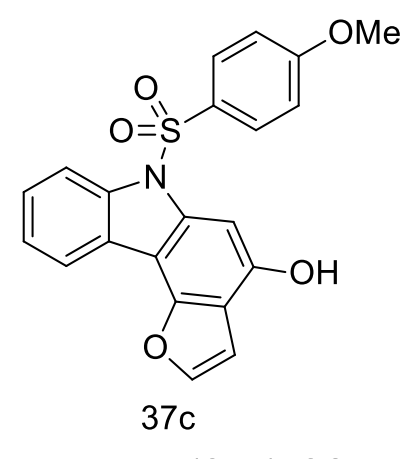

$500 \mathrm{MHz},(\mathrm{CD} 3) 2 \mathrm{CO}$

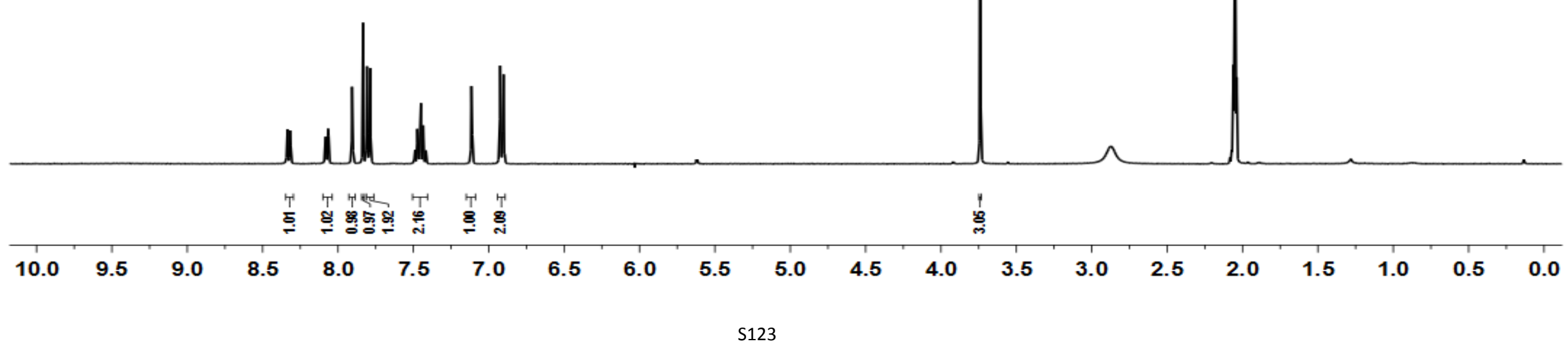




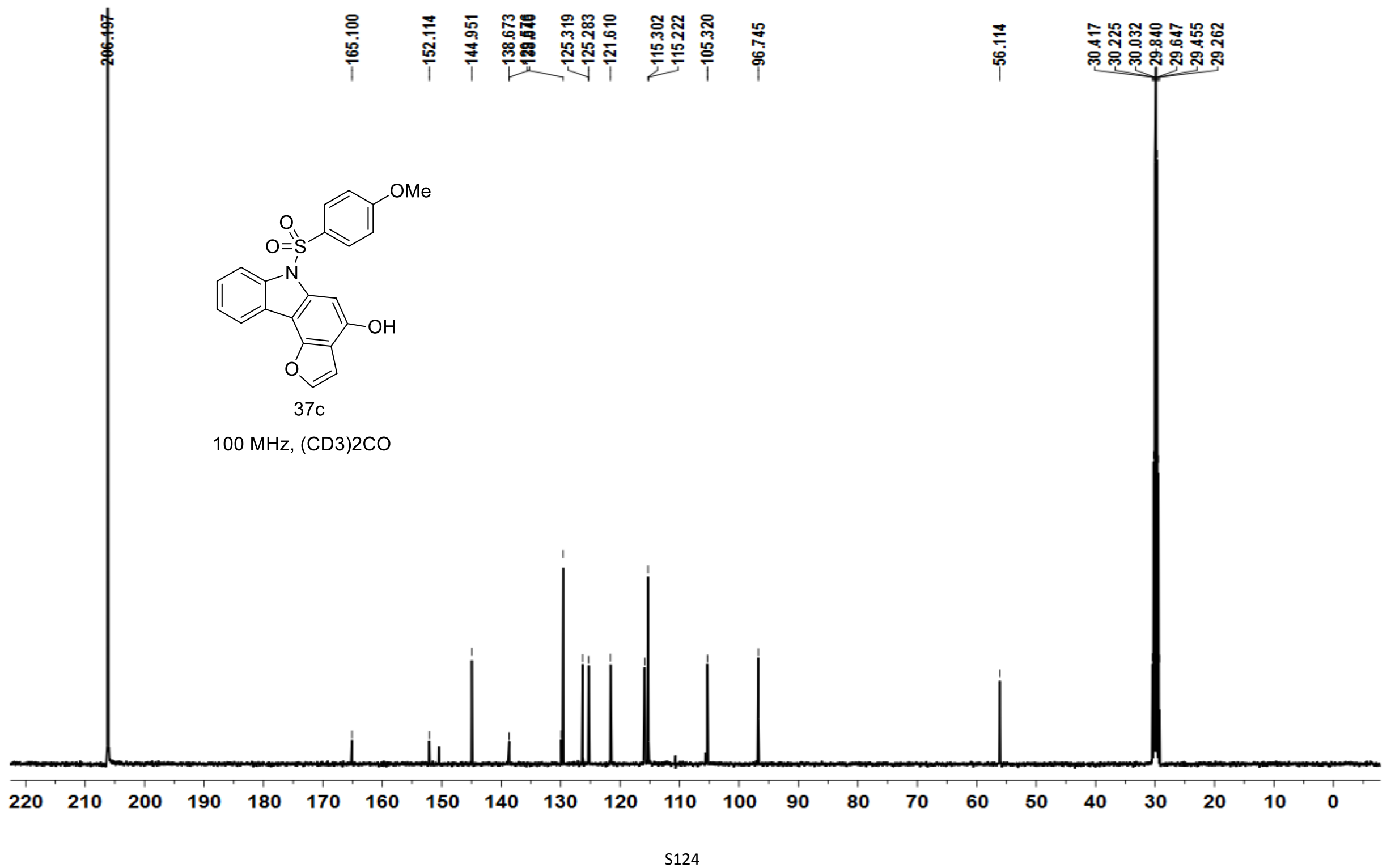




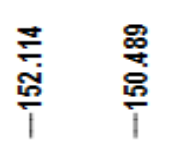

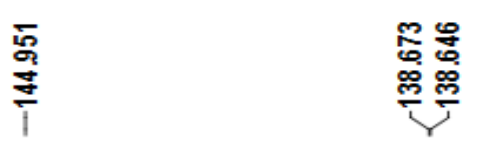

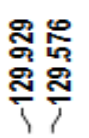

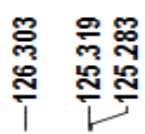

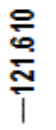

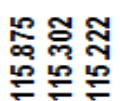

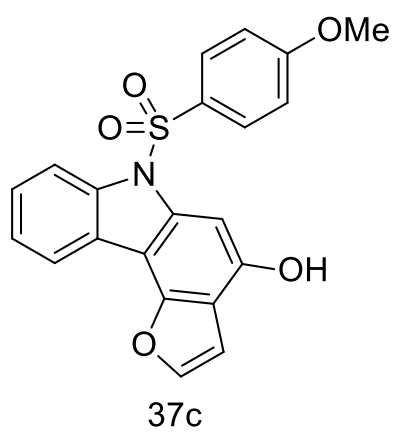

$100 \mathrm{MHz},(\mathrm{CD} 3) 2 \mathrm{CO}$

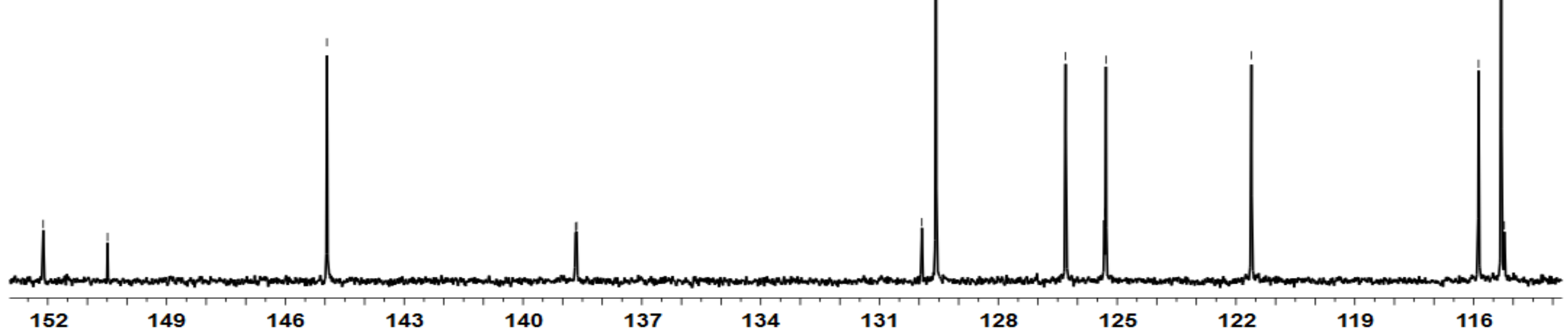




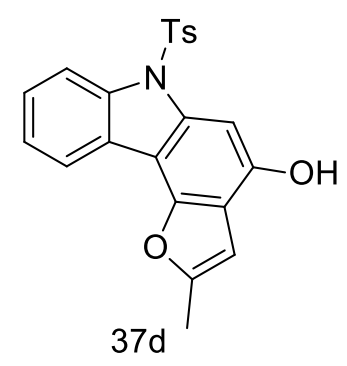

$500 \mathrm{MHz},(\mathrm{CD} 3) 2 \mathrm{CO}$

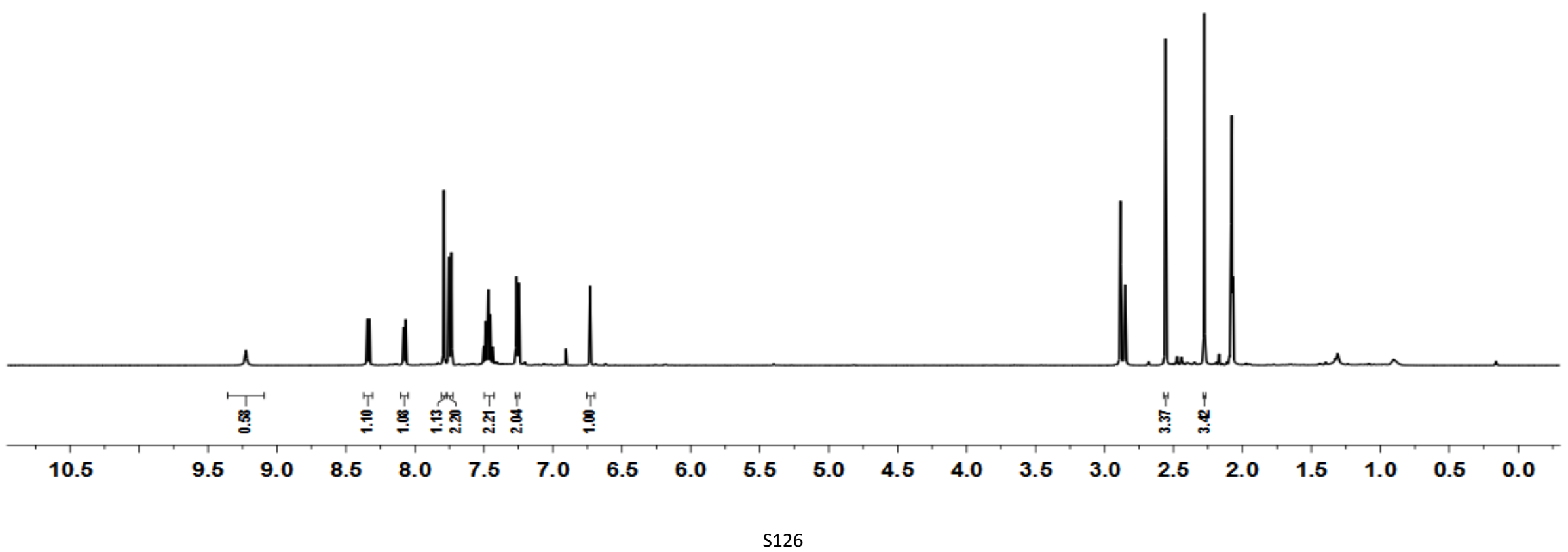




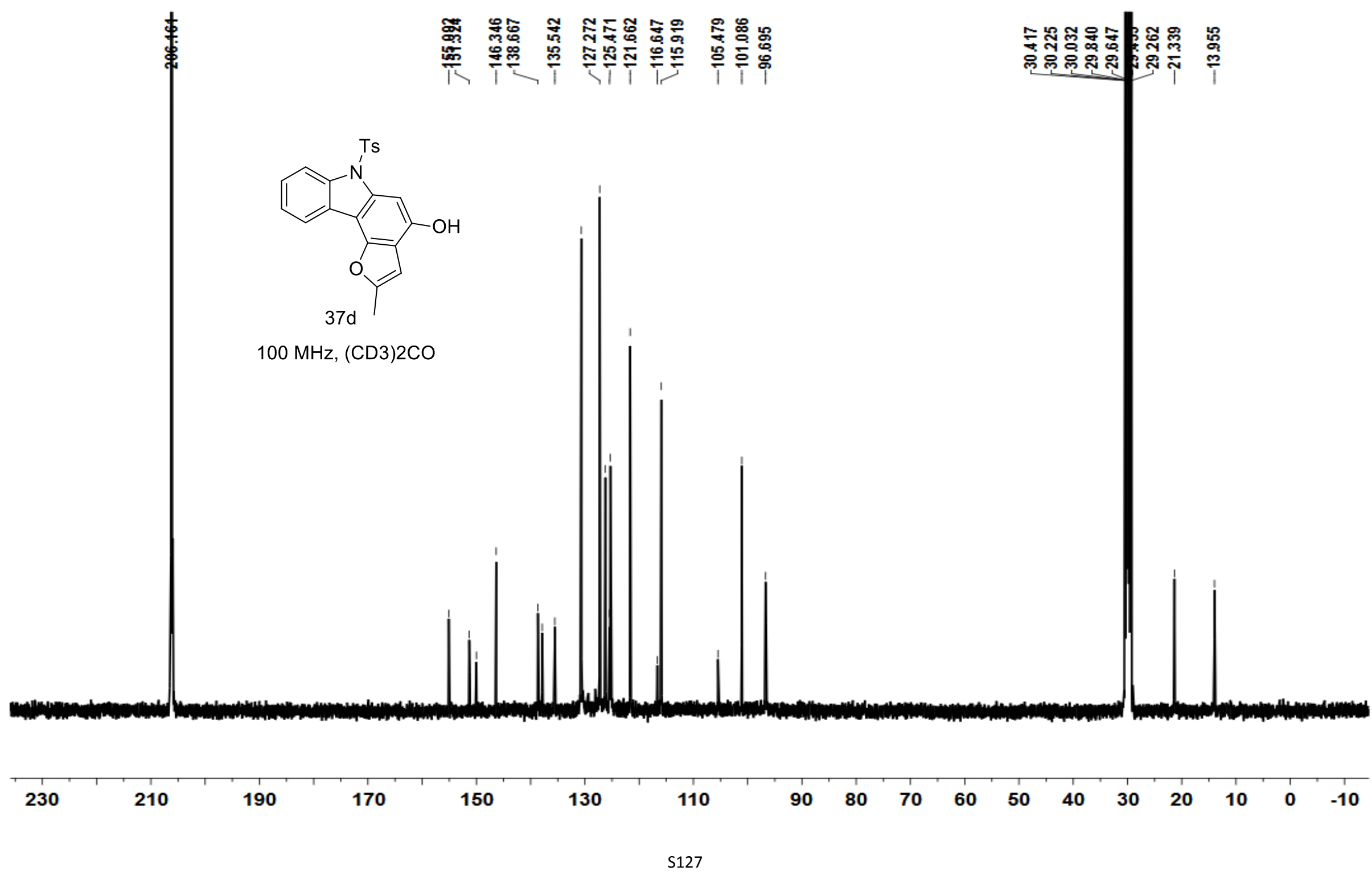




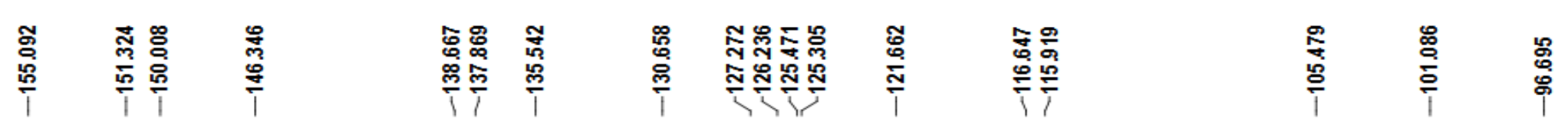

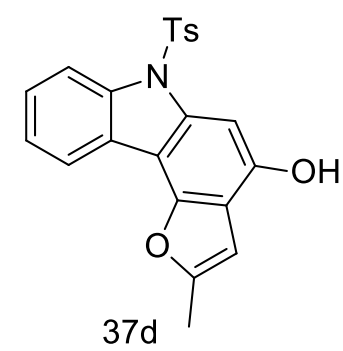

$100 \mathrm{MHz},(\mathrm{CD} 3) 2 \mathrm{CO}$

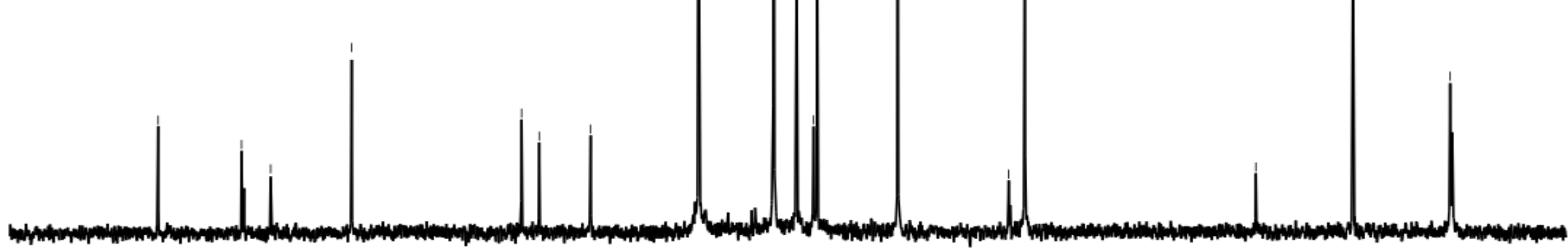

$160 \quad 155 \quad 150$

145

140

135

130

125

120

115

110

105

100

95 


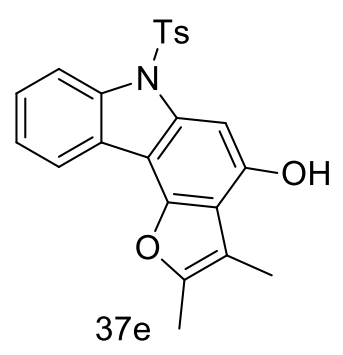

$500 \mathrm{MHz}, \mathrm{CDCl} 3$

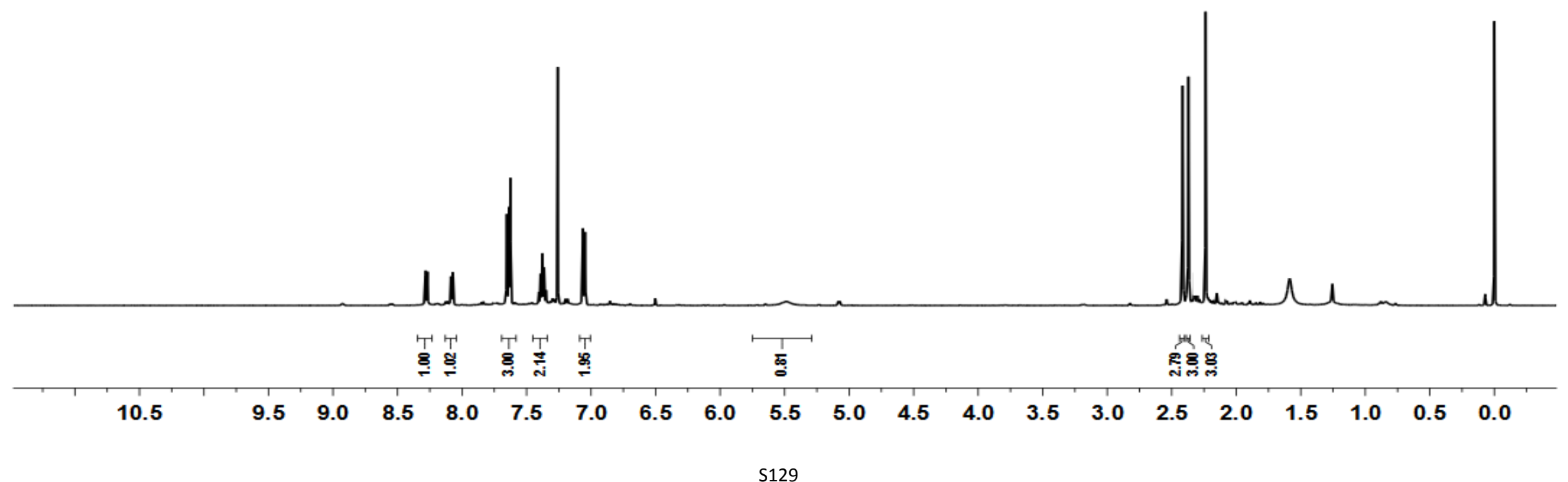




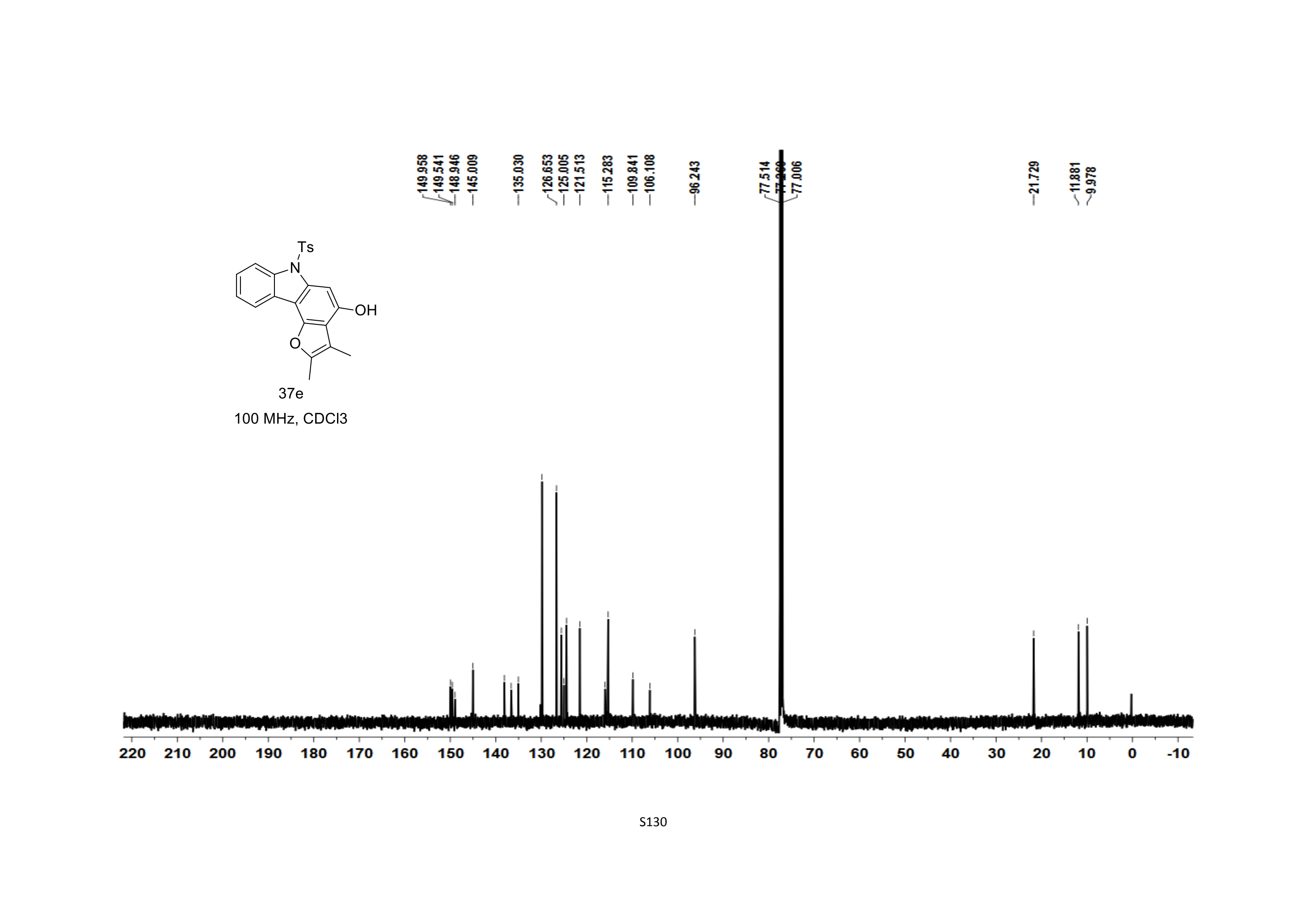




\begin{tabular}{|c|c|c|c|c|c|c|c|}
\hline 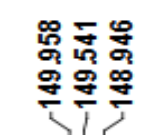 & 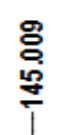 & 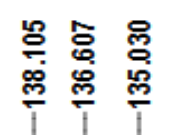 & $\begin{array}{l}\overline{0} \\
\text { a. } \\
\text { a }\end{array}$ & 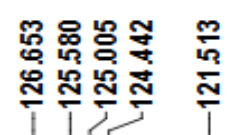 & 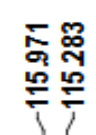 & 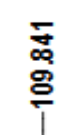 & $\frac{\circ}{\circ}$ \\
\hline
\end{tabular}

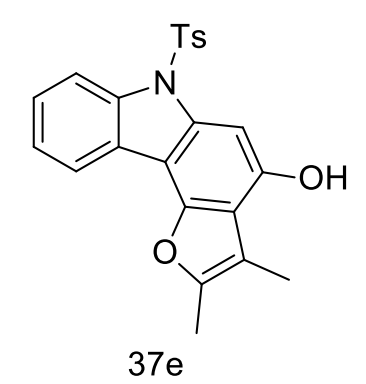

$100 \mathrm{MHz}, \mathrm{CDCl} 3$

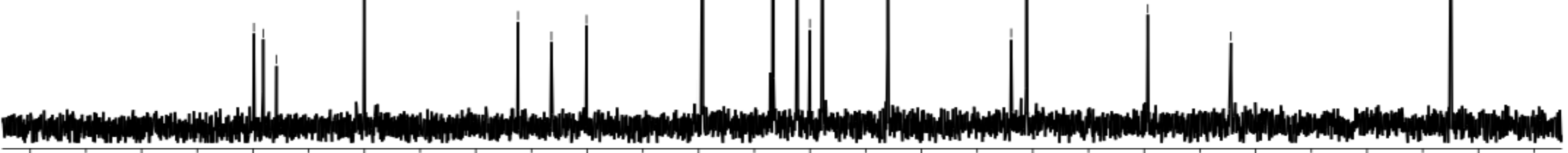




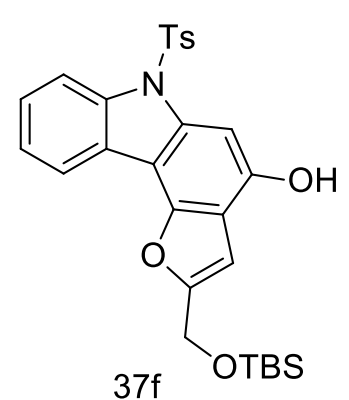

$500 \mathrm{MHz}, \mathrm{CDCl} 3$

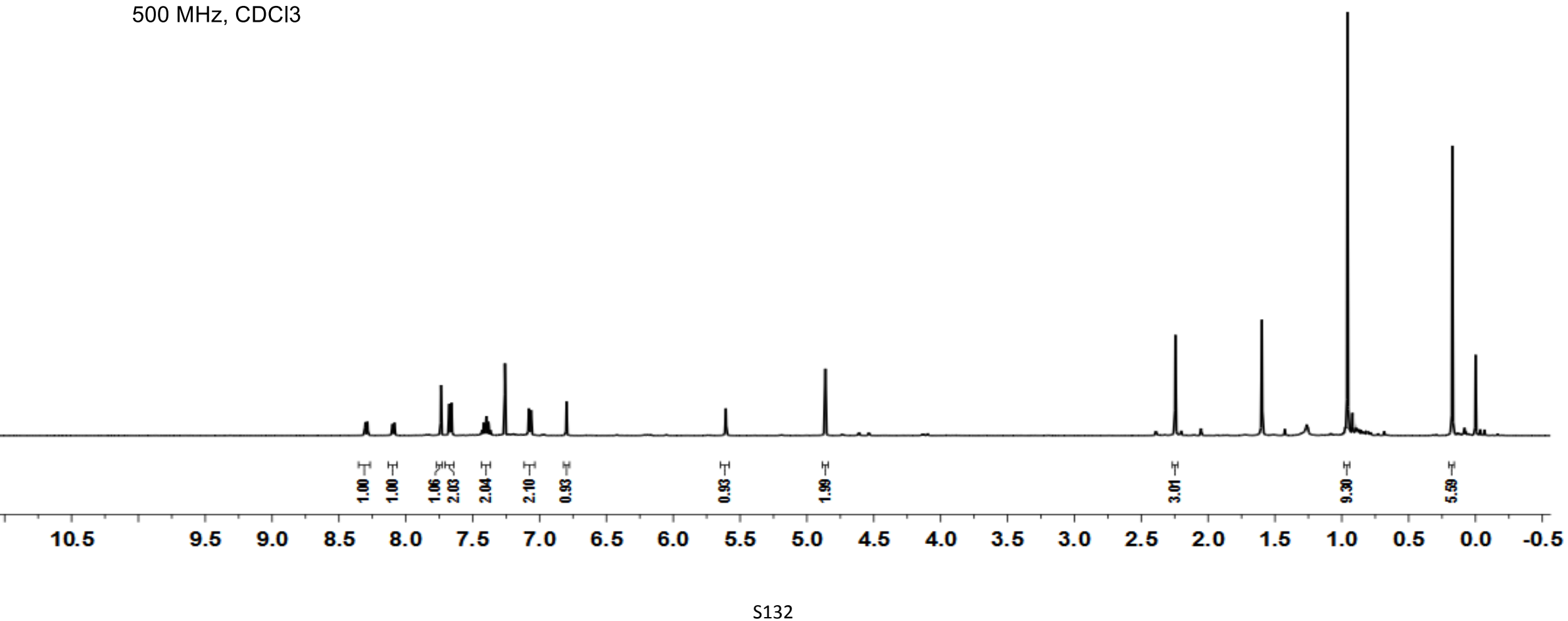




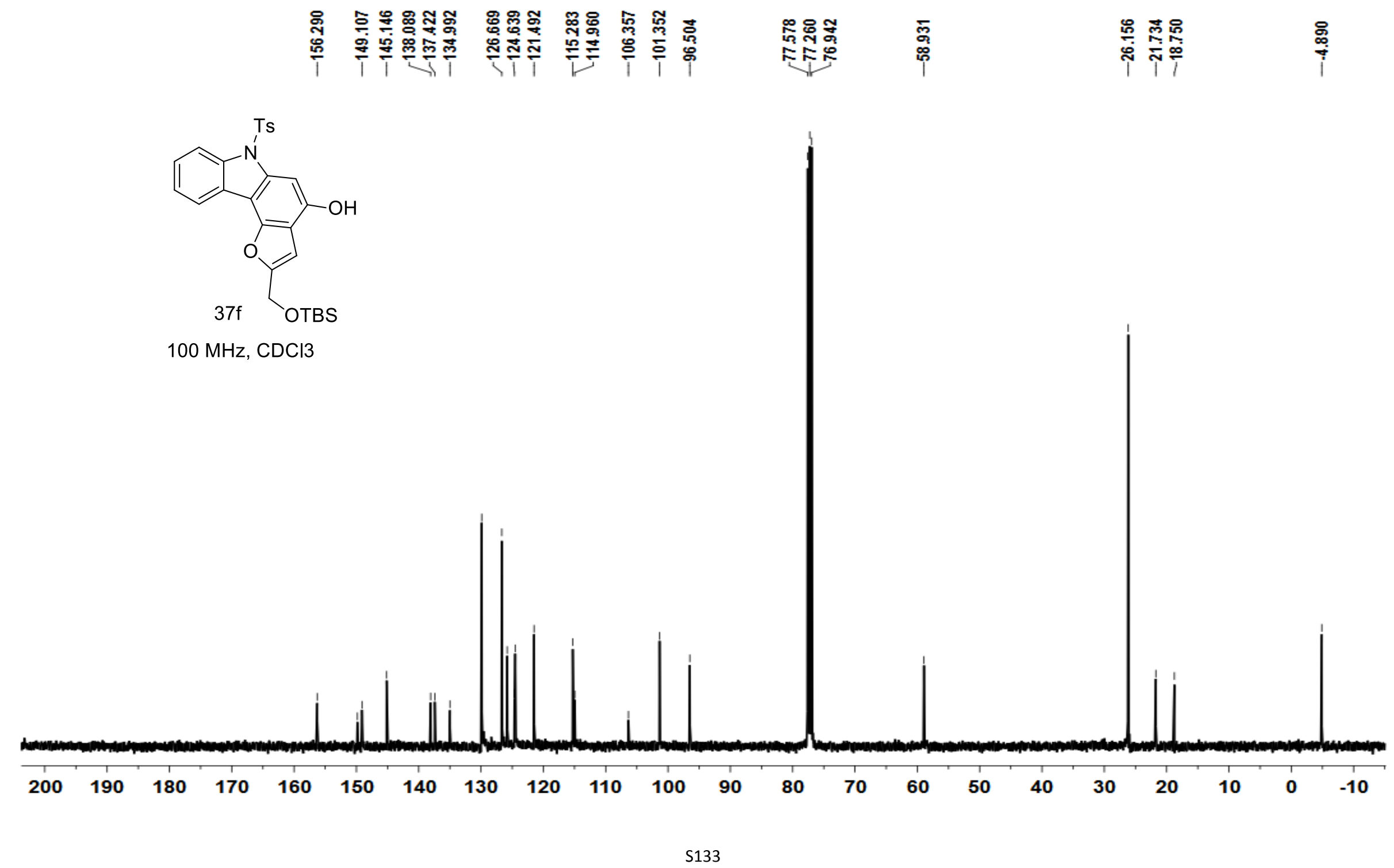




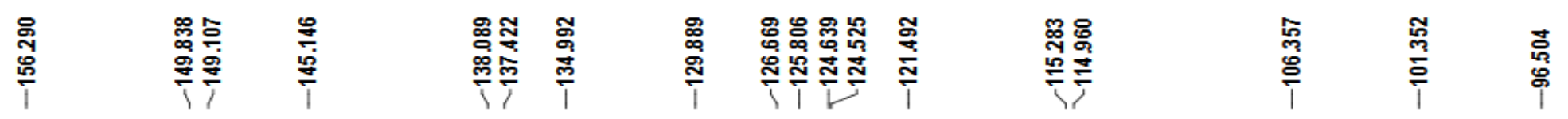

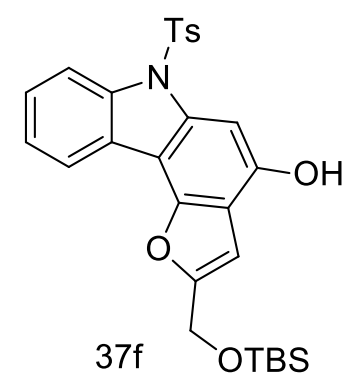

$100 \mathrm{MHz}, \mathrm{CDCl} 3$

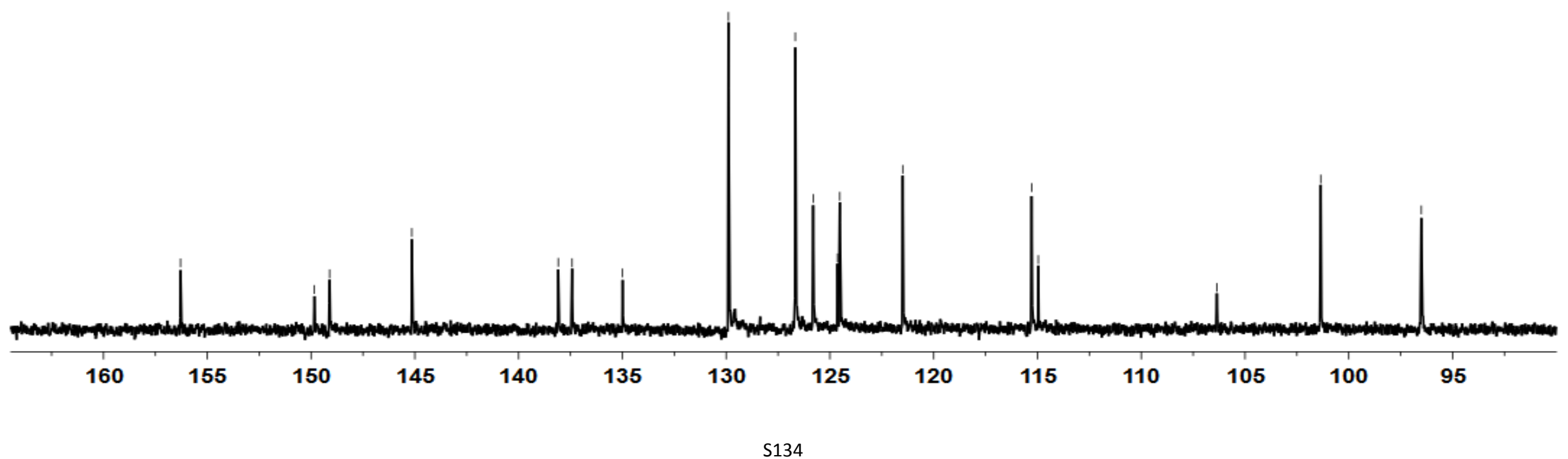




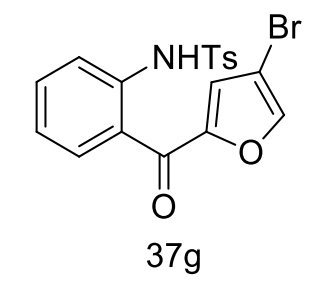

$500 \mathrm{MHz}, \mathrm{CDCl} 3$

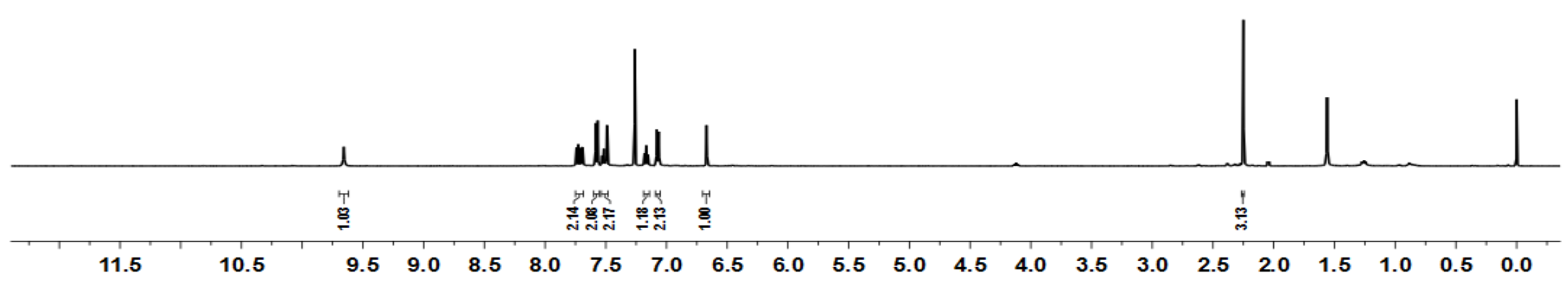




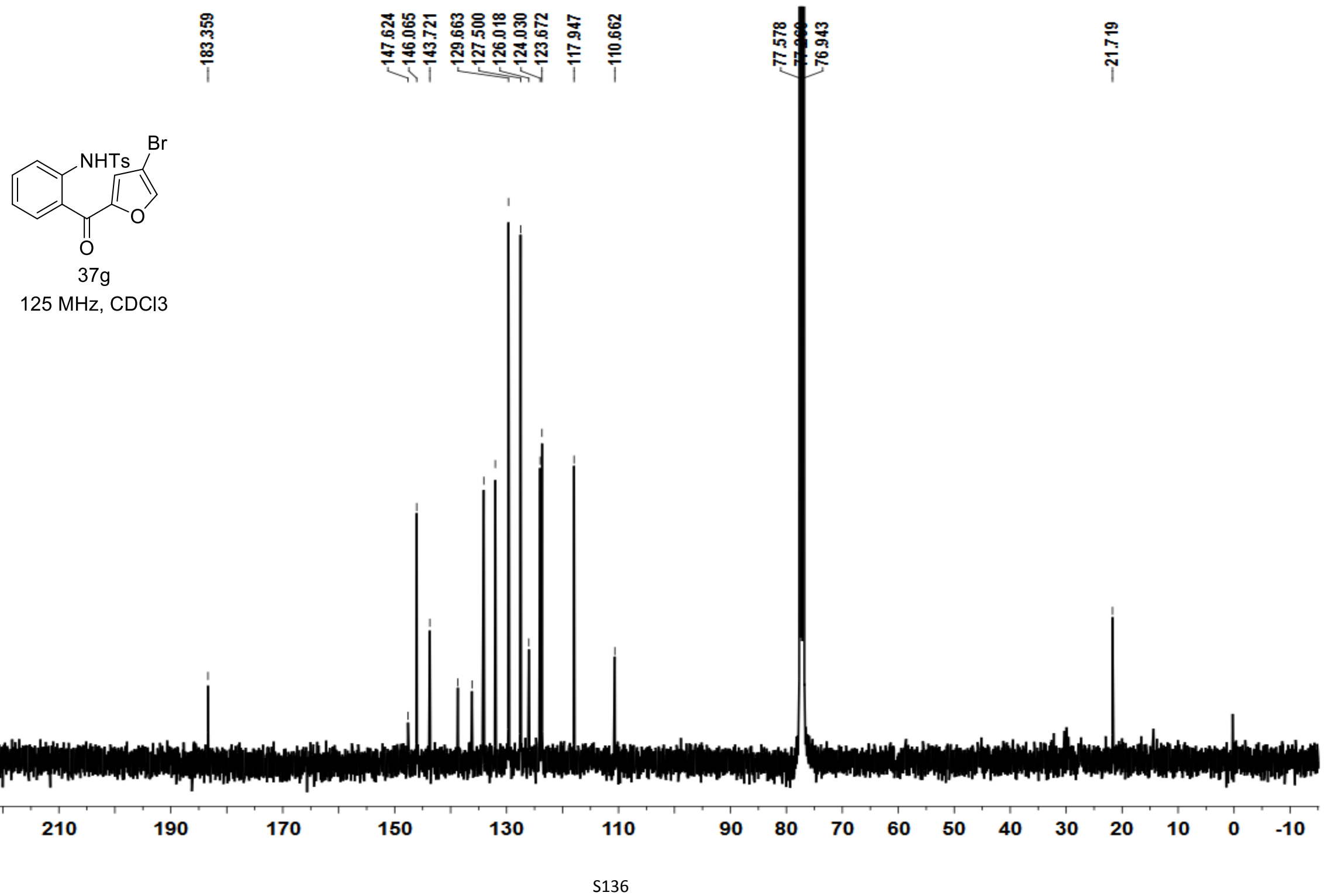




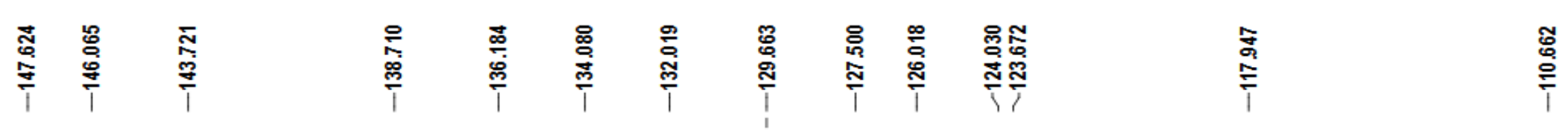

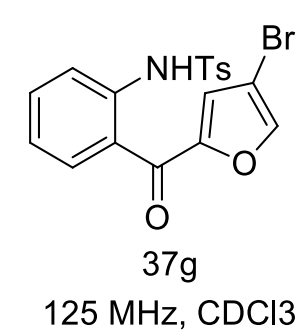

$125 \mathrm{MHz}, \mathrm{CDCl} 3$

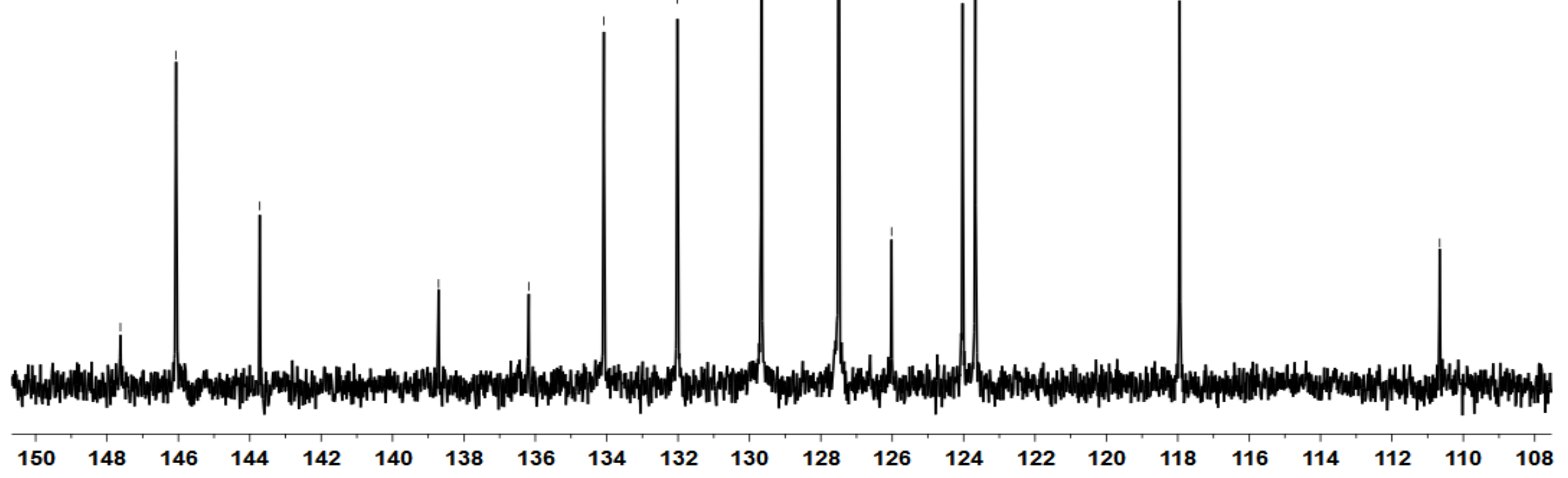




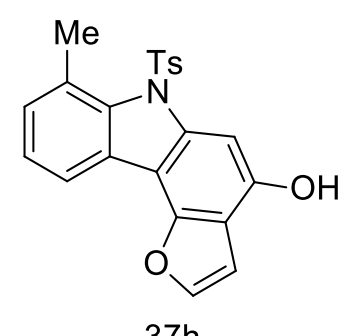

$500 \mathrm{MHz}, \mathrm{CDCl} 3$

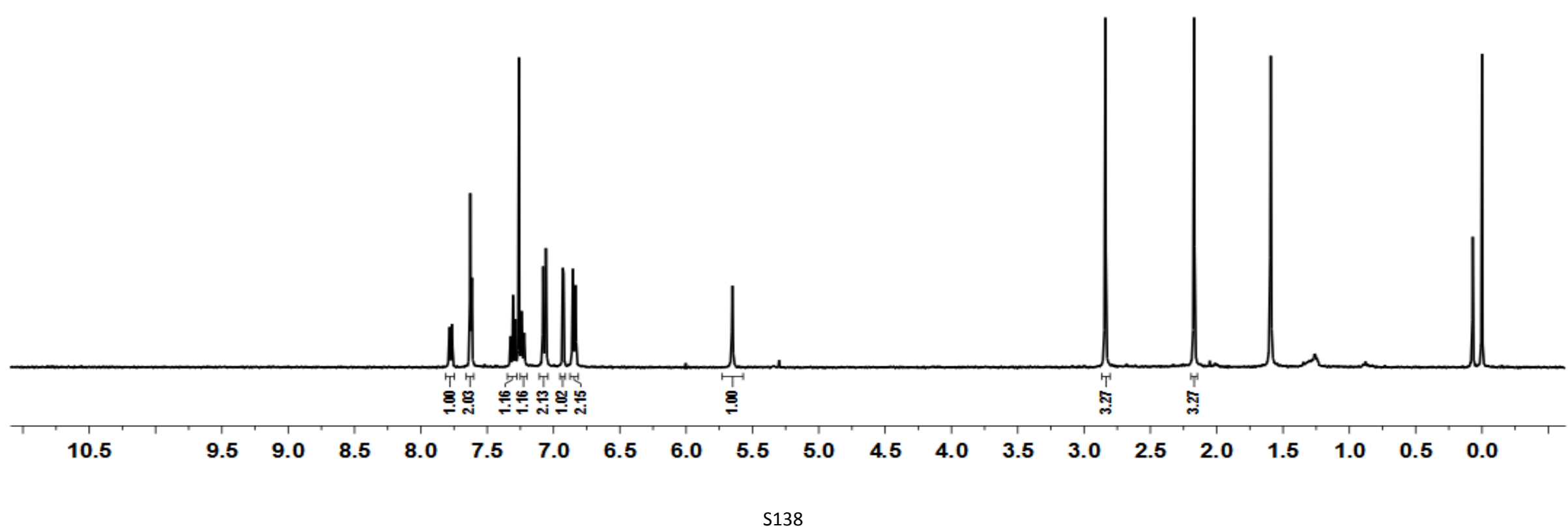




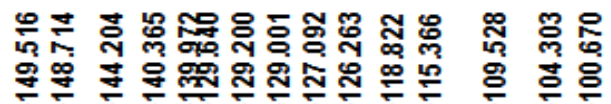 \\ 남}

울

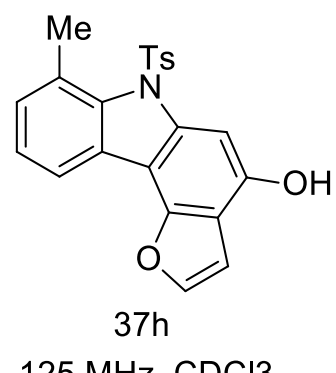

$125 \mathrm{MHz}, \mathrm{CDCl} 3$

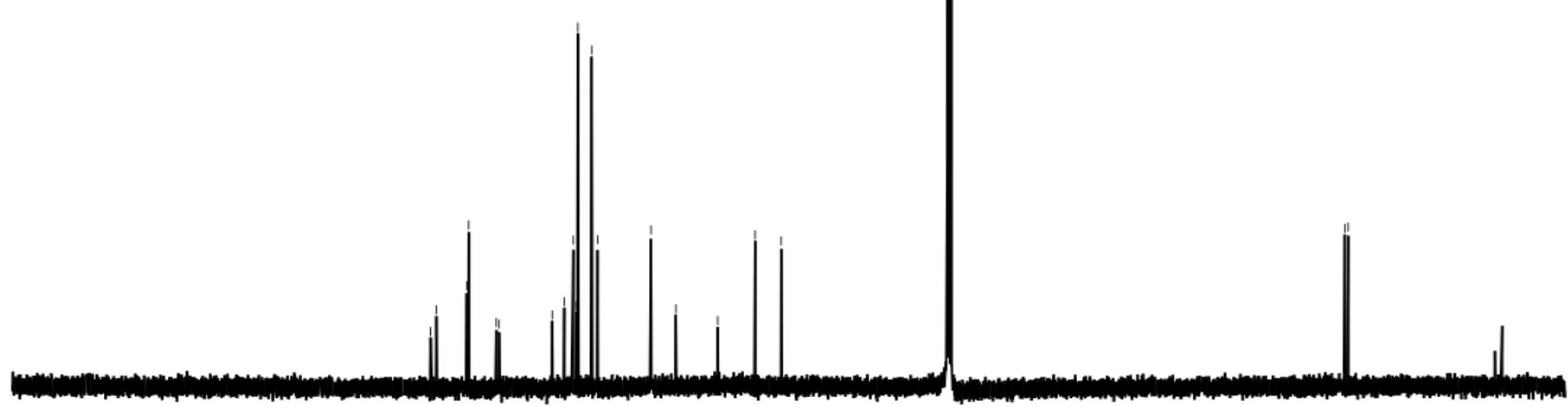

$\begin{array}{lllllllllll}200 & 190 & 180 & 170 & 160 & 150 & 140 & 130 & 120 & 110 & 100\end{array}$

9080

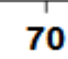

60

50

40

30

20 


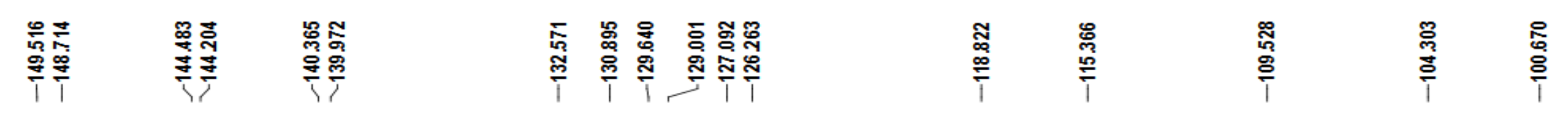

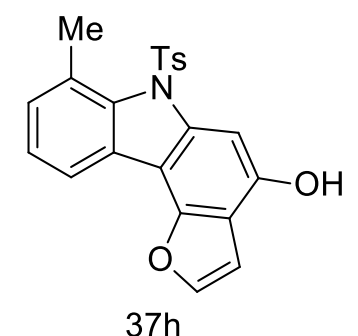

$125 \mathrm{MHz}, \mathrm{CDCl} 3$

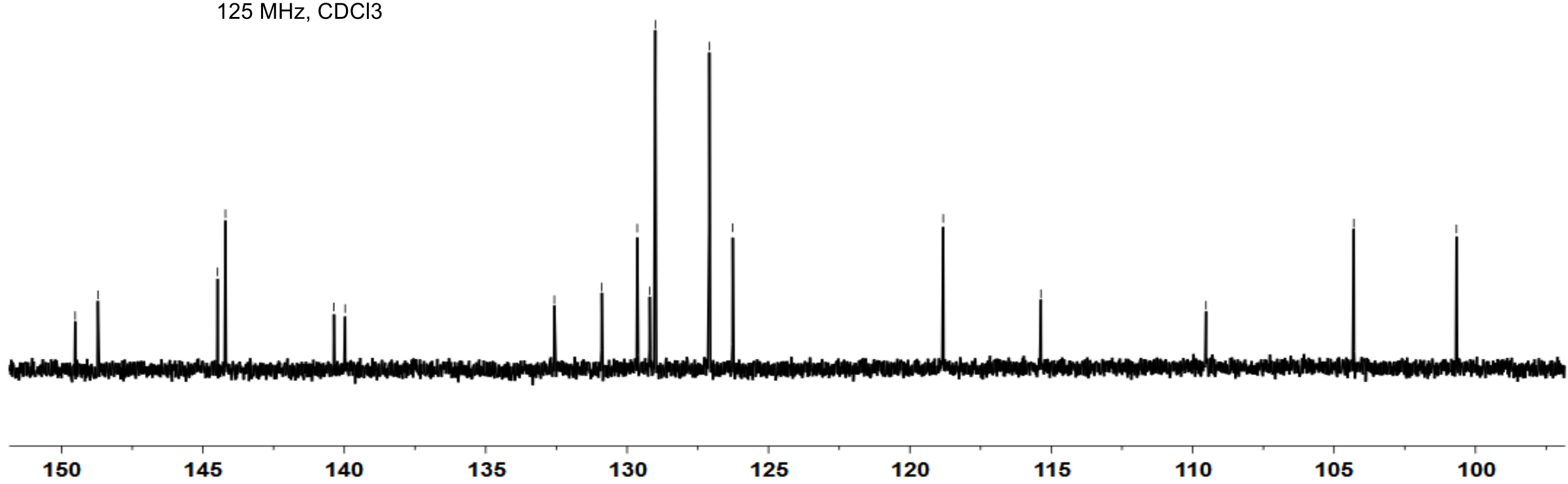




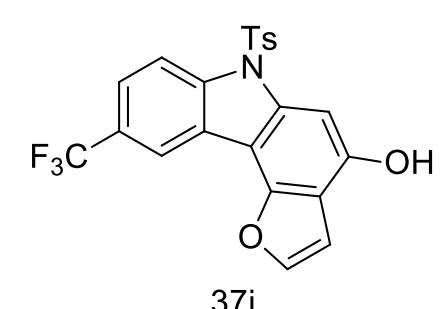

$500 \mathrm{MHz},(\mathrm{CD} 3) 2 \mathrm{CO}$

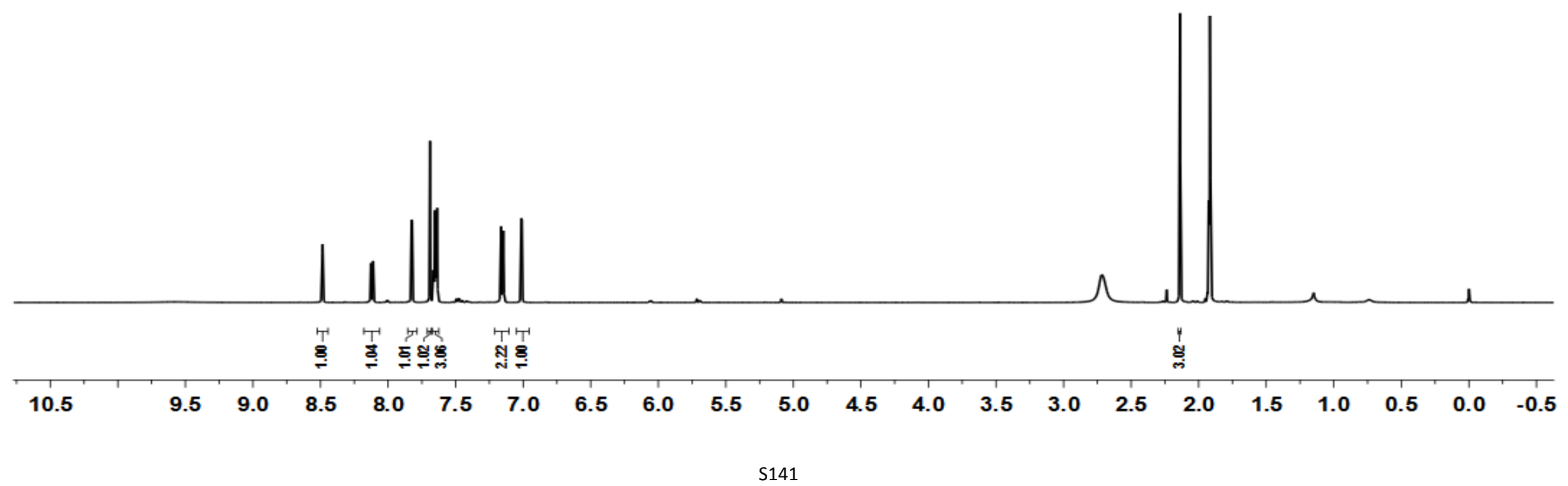




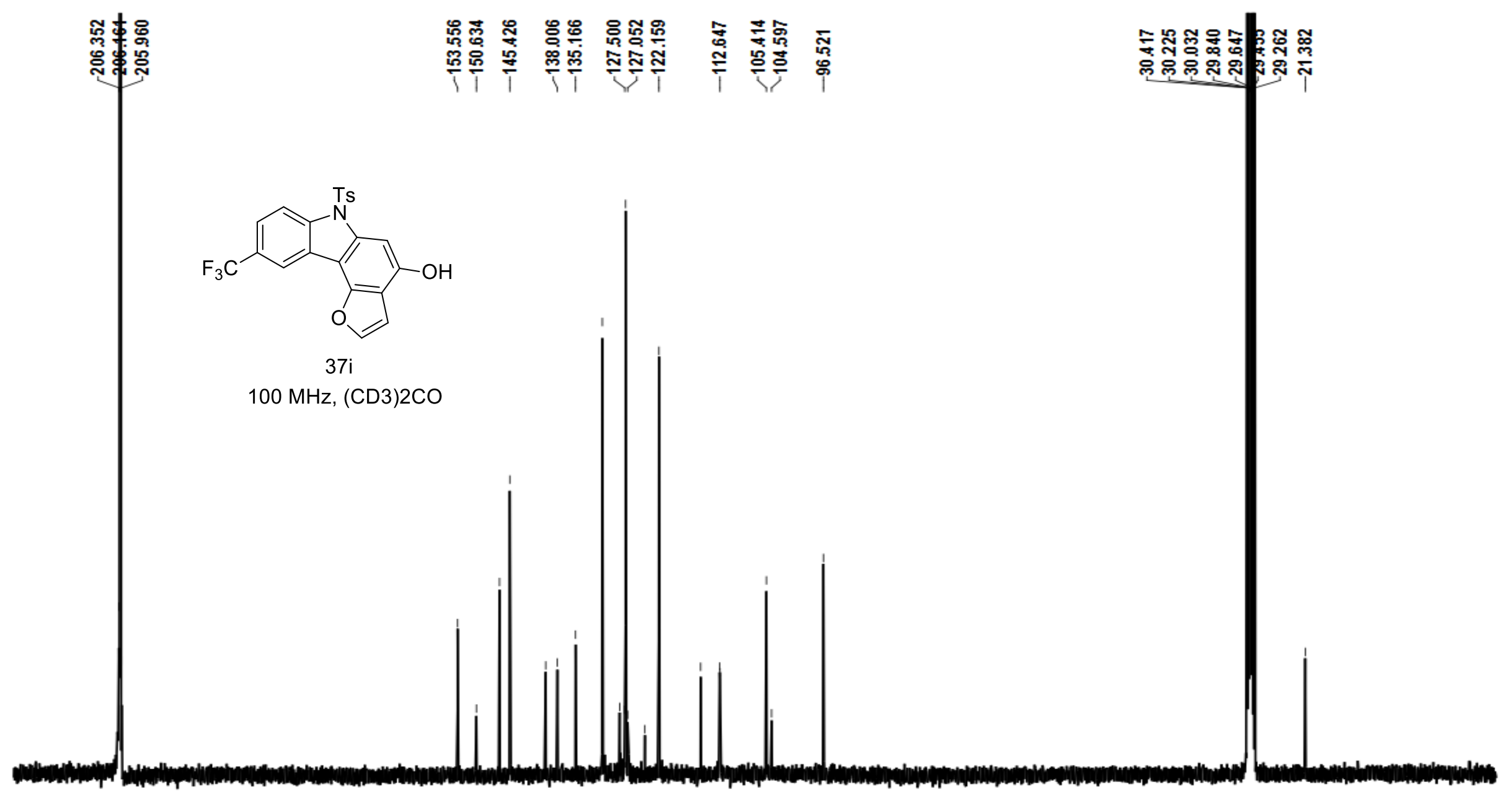

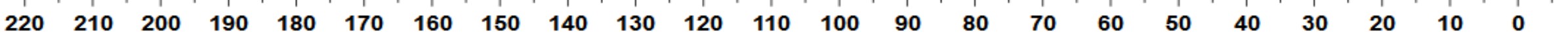



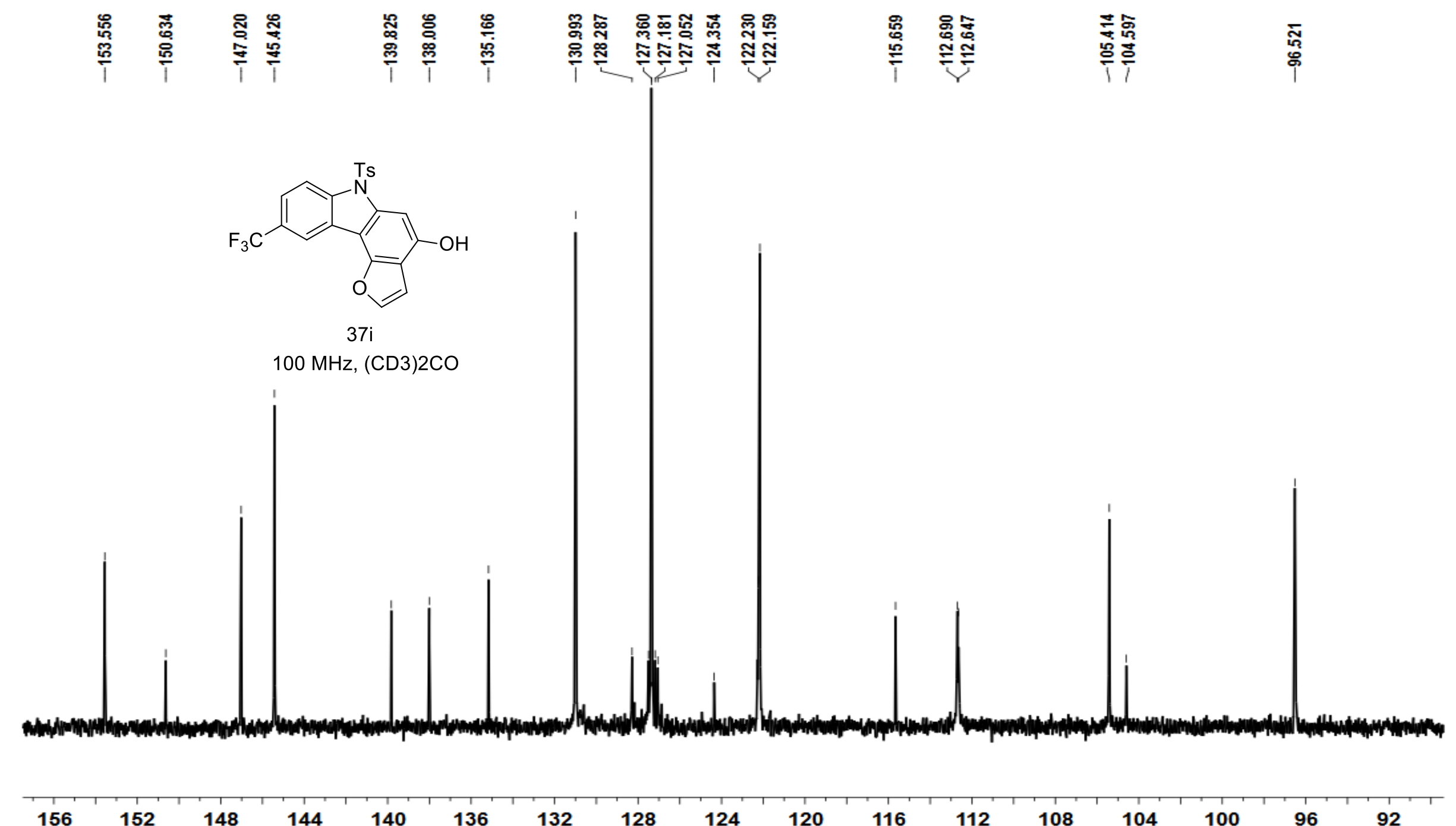

124

116

112 


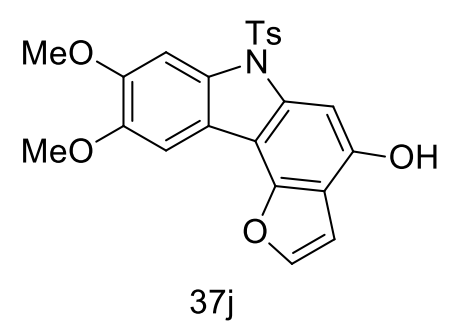

$400 \mathrm{MHz}, \mathrm{CDCl} 3$

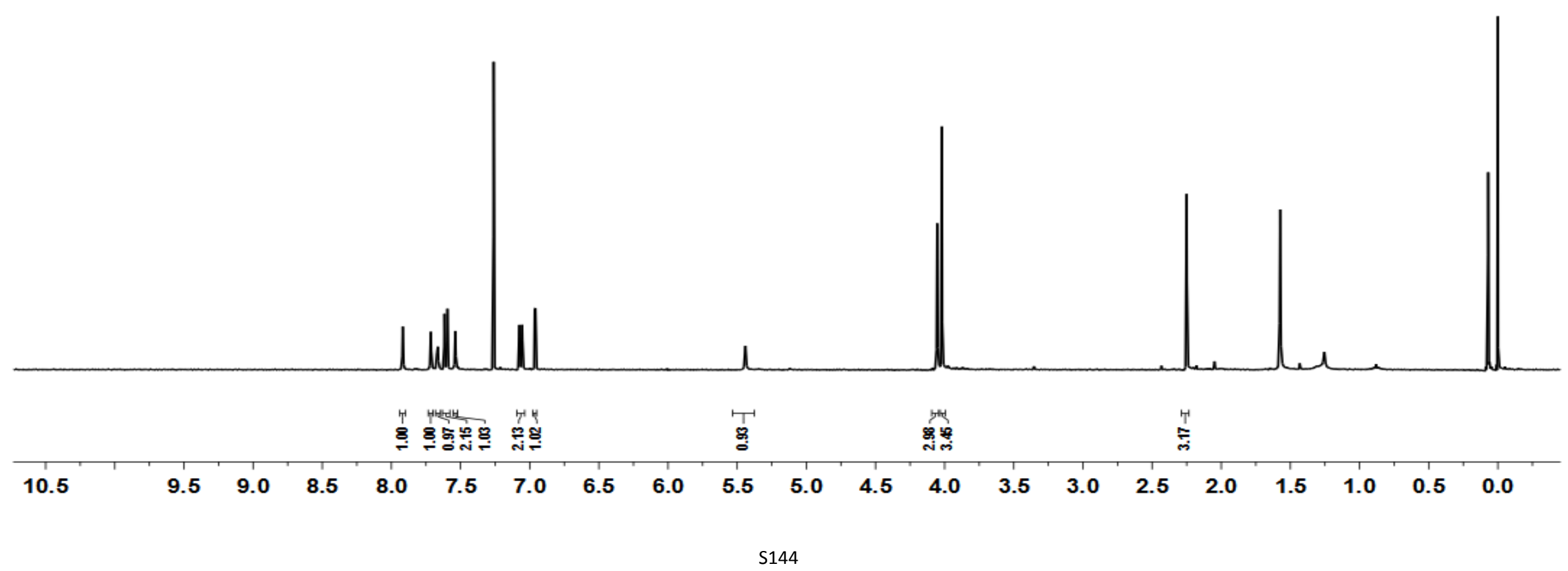



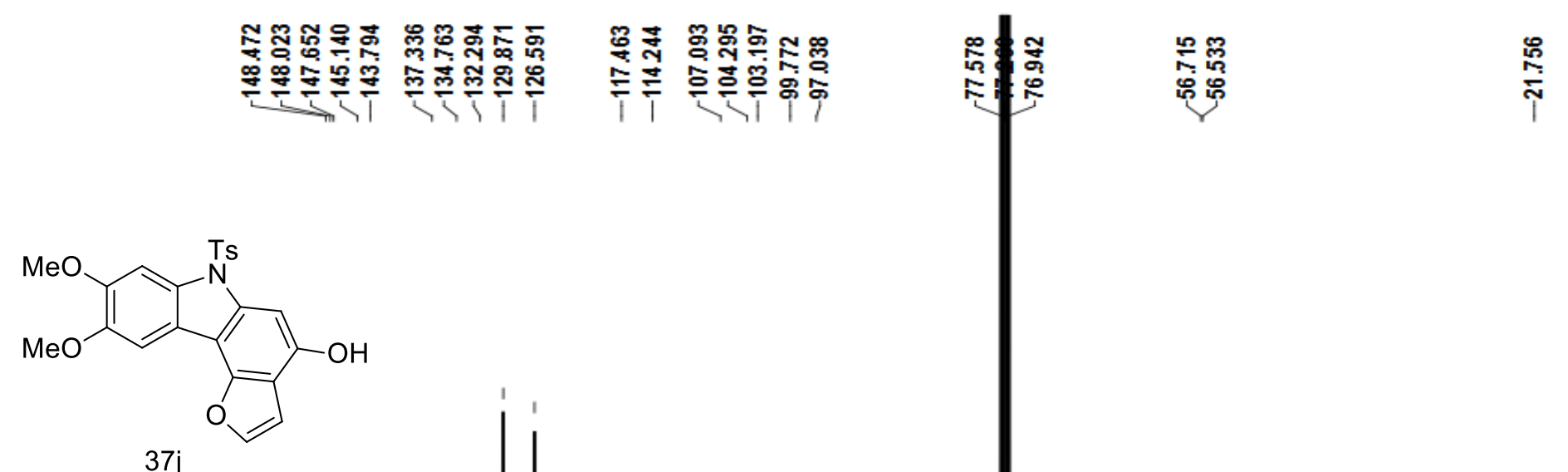

$125 \mathrm{MHz}, \mathrm{CDCl} 3$

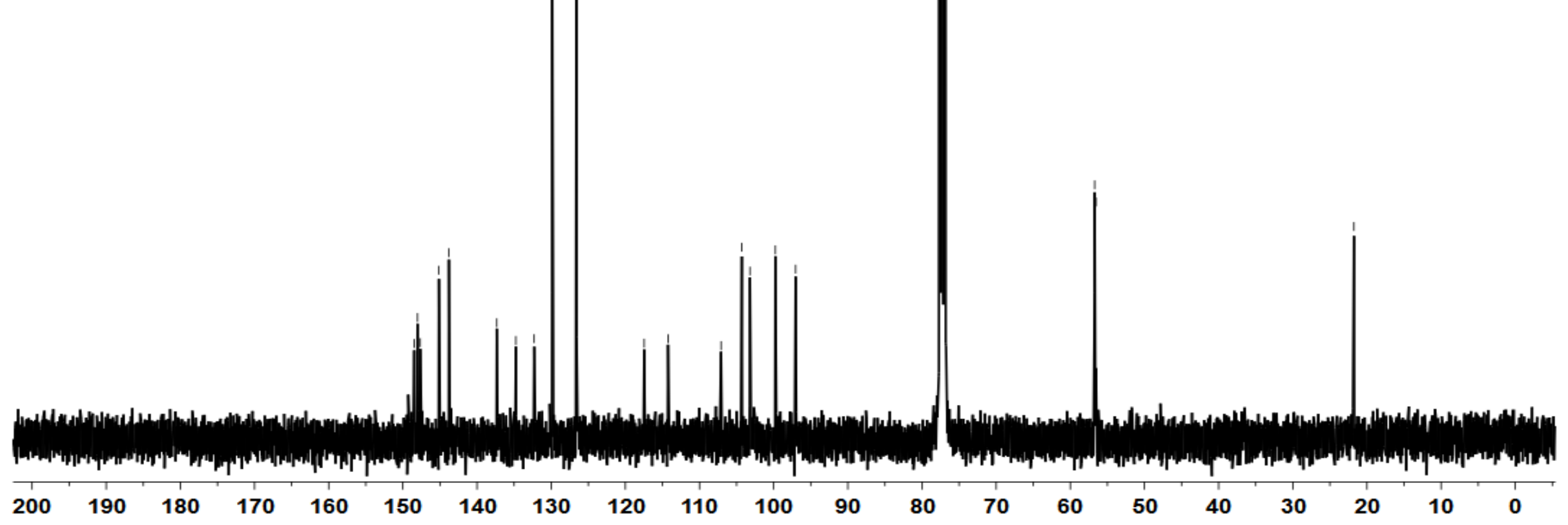




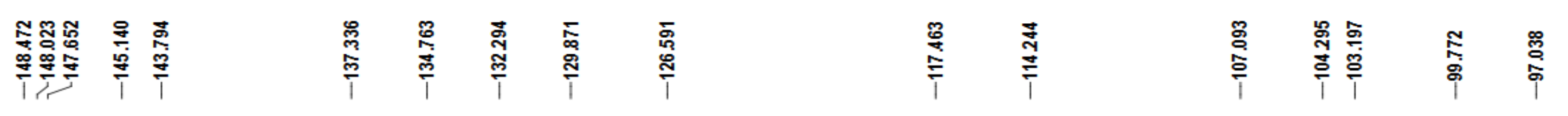

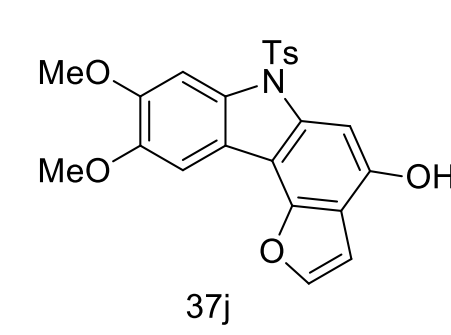

$125 \mathrm{MHz}, \mathrm{CDCl} 3$

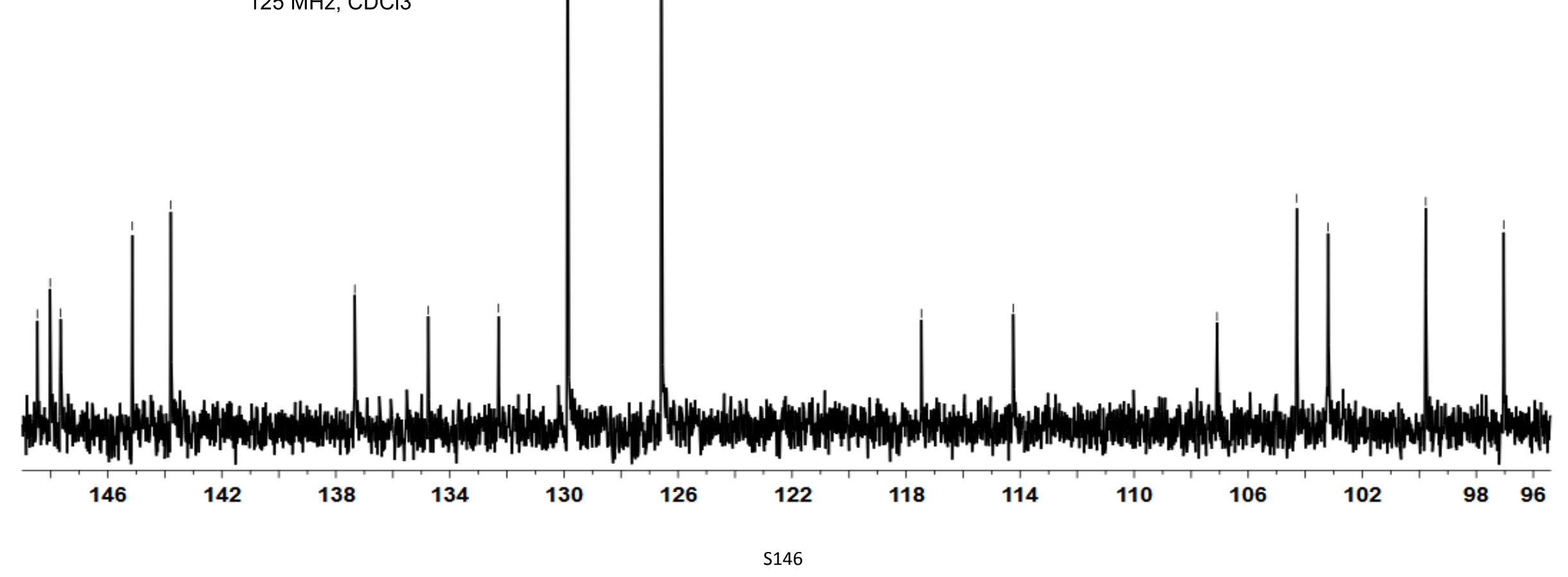




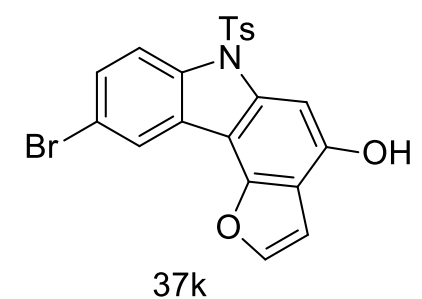

$500 \mathrm{MHz},(C D 3) 2 \mathrm{CO}$

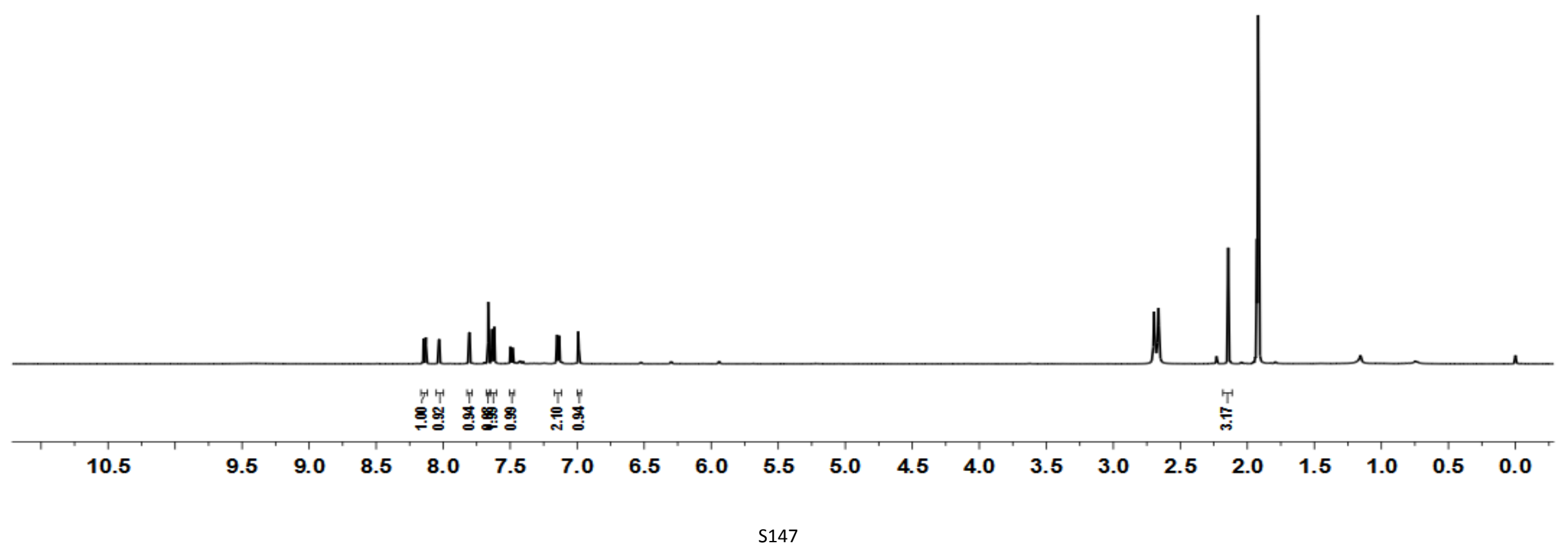




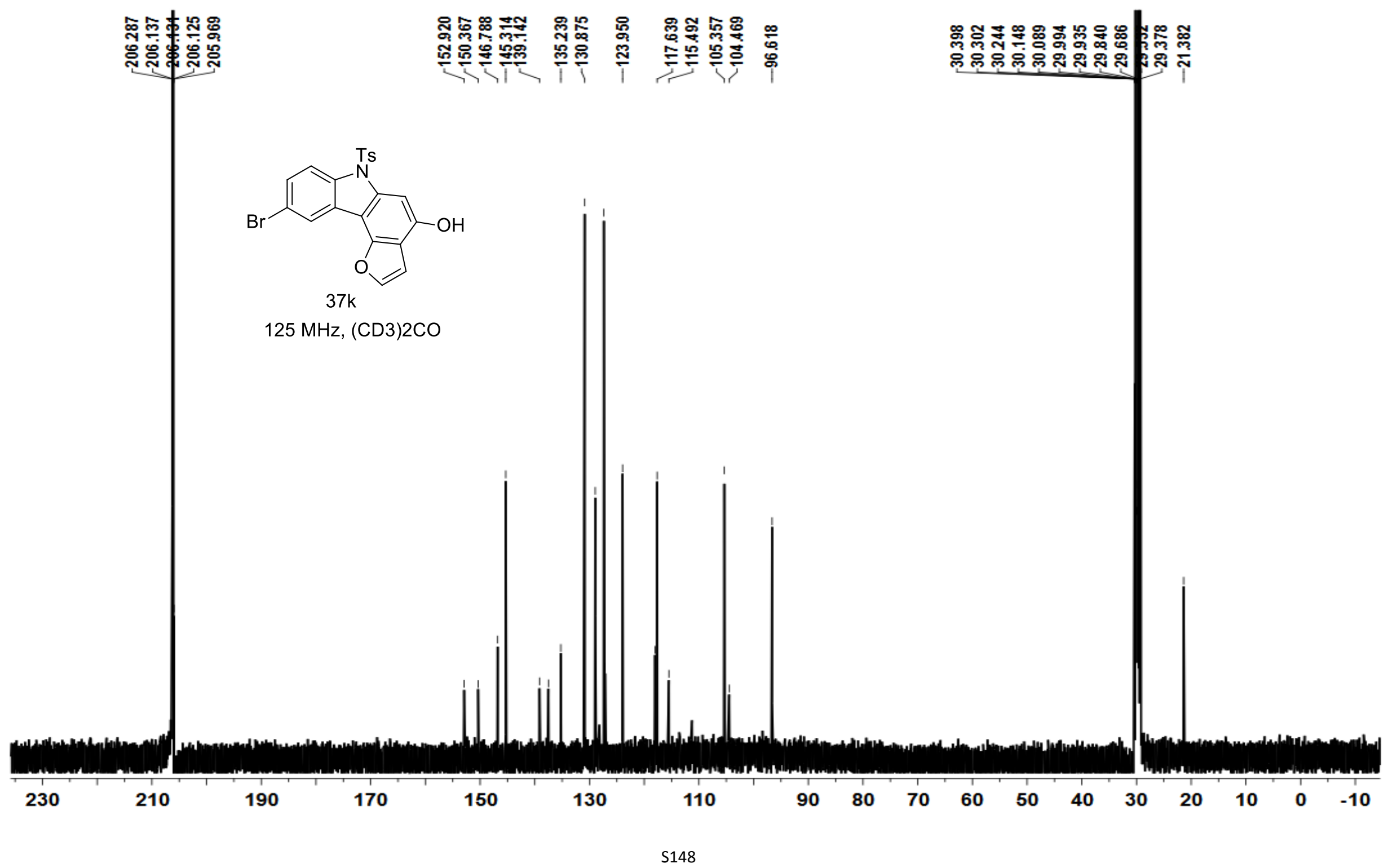




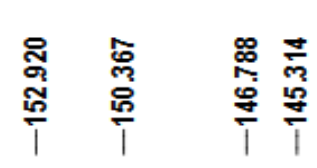

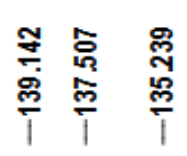

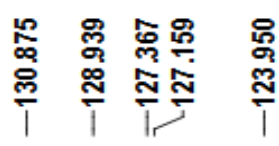

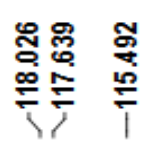

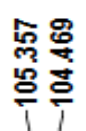
$\stackrel{\infty}{\circ}$

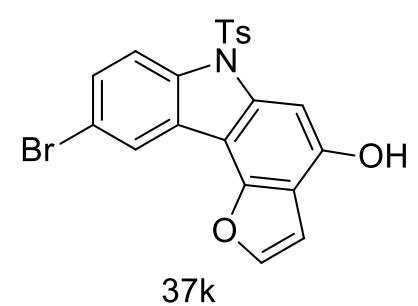

$125 \mathrm{MHz},(\mathrm{CD} 3) 2 \mathrm{CO}$

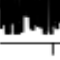

150

145

140

135

130

125

120

115

110

105

100

95 


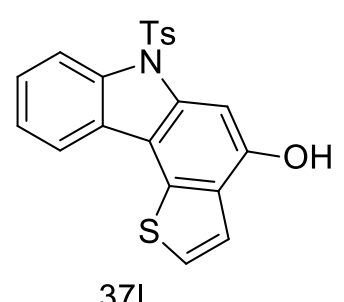

$400 \mathrm{MHz},(C D 3) 2 \mathrm{CO}$

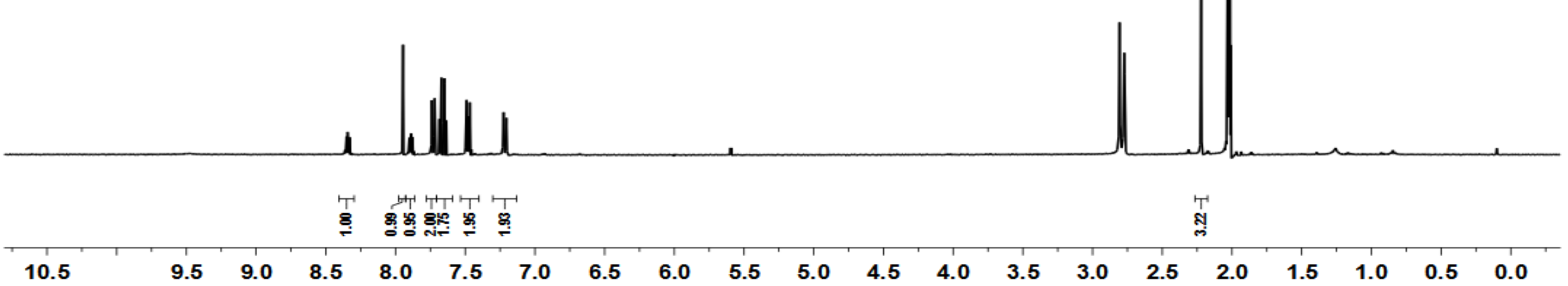




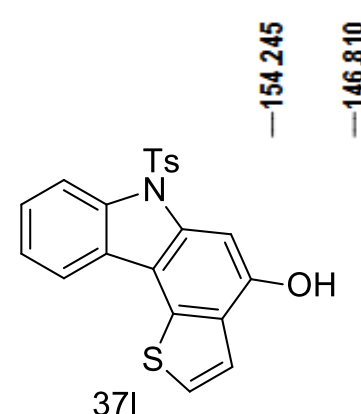

$125 \mathrm{MHz}, \mathrm{CD} 3 \mathrm{OD}$

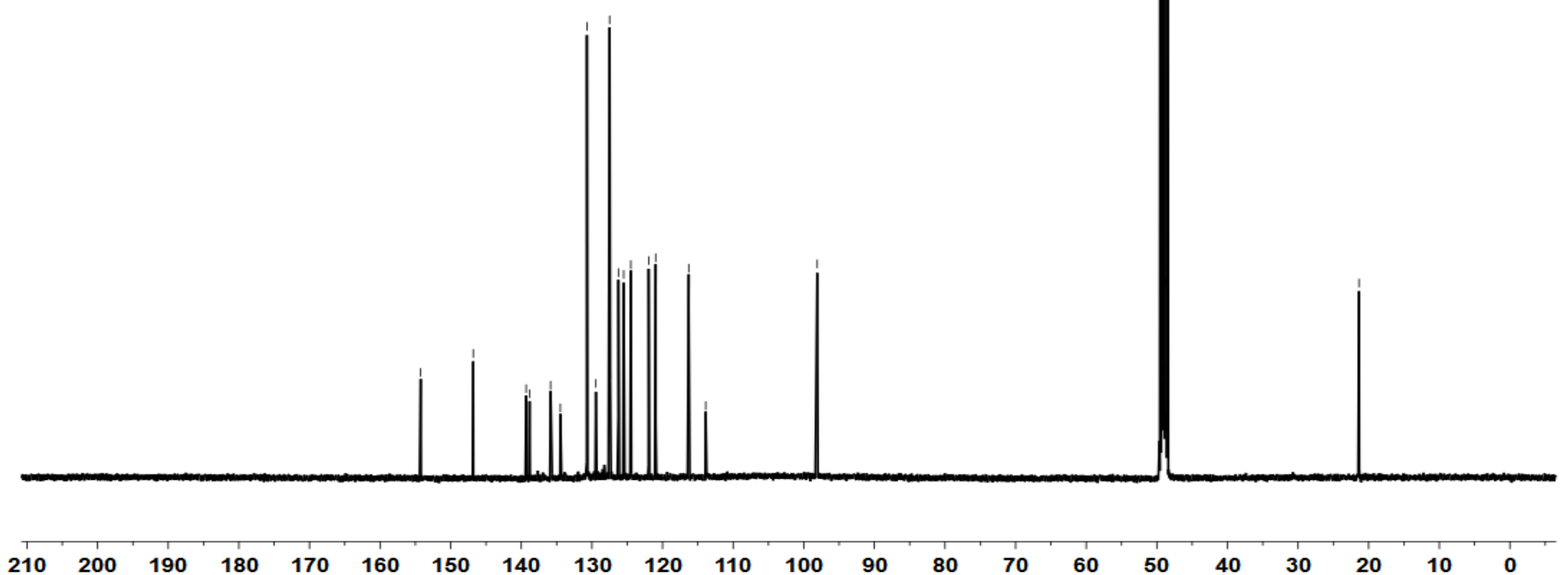




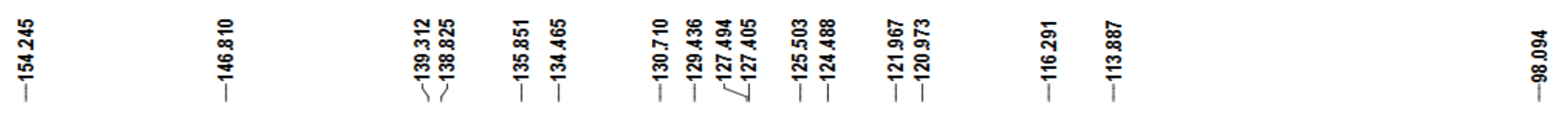

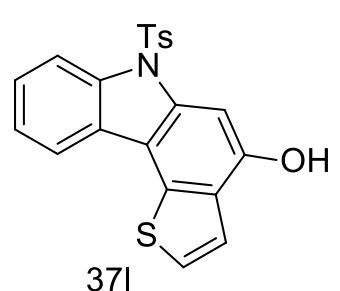

$125 \mathrm{MHz}, \mathrm{CD} 3 \mathrm{OD}$

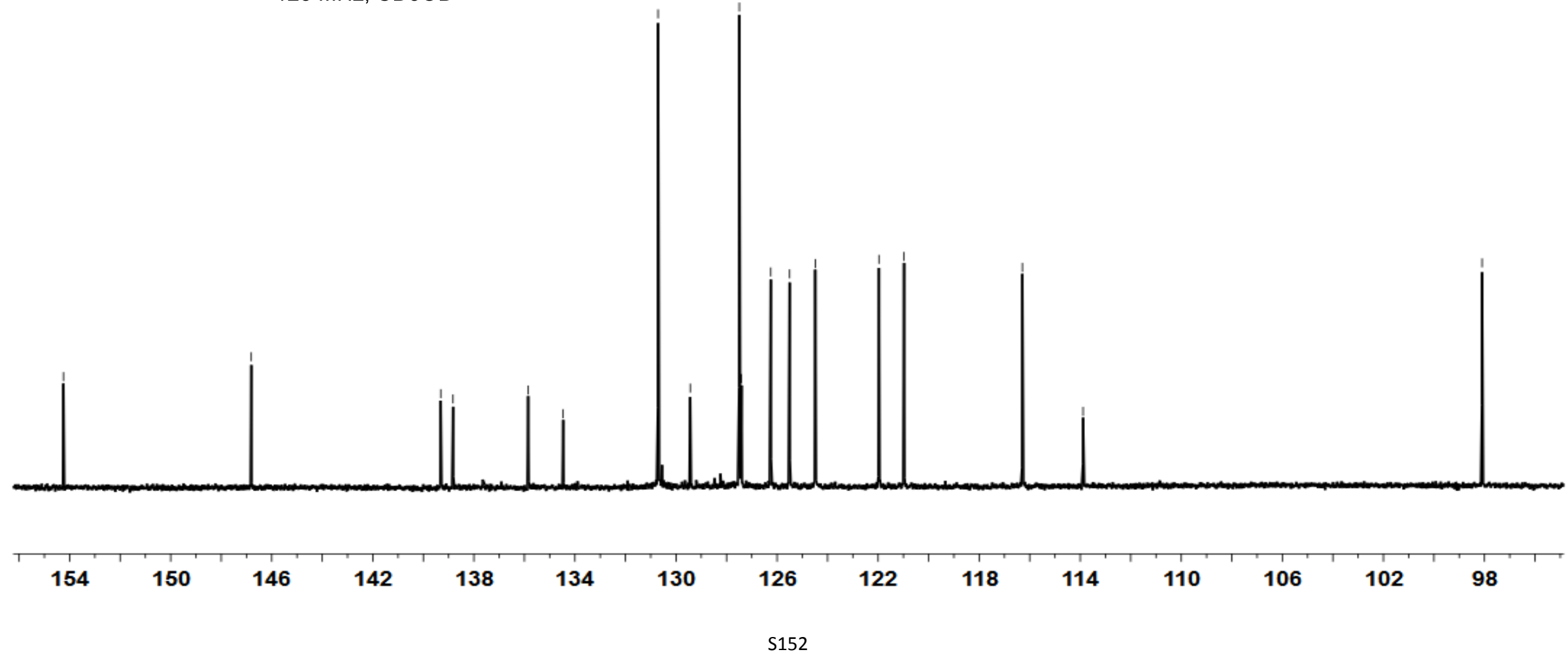




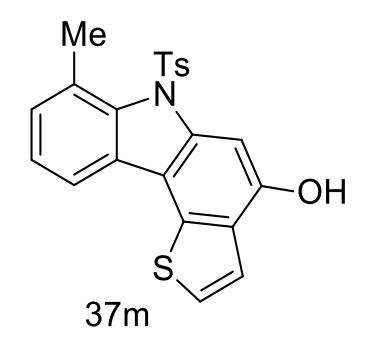

$400 \mathrm{MHz}, \mathrm{CDCl} 3$

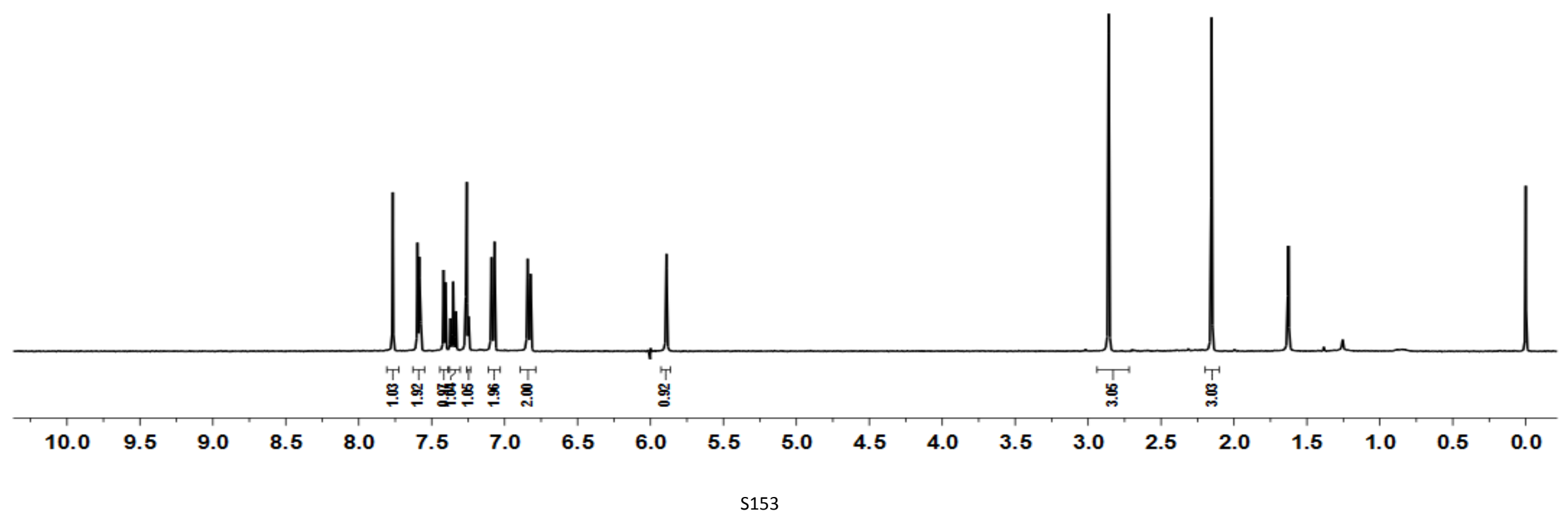




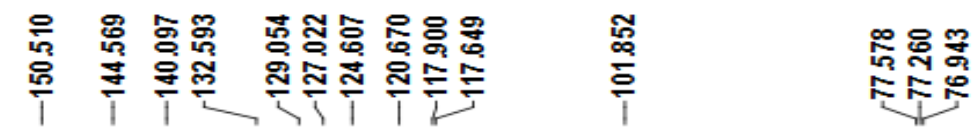

సี:

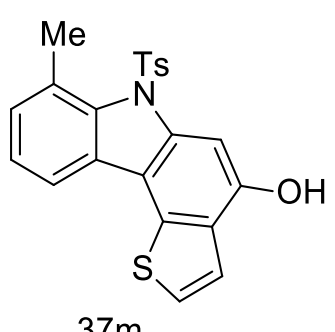

$37 \mathrm{~m}$

$100 \mathrm{MHz}, \mathrm{CDCl} 3$

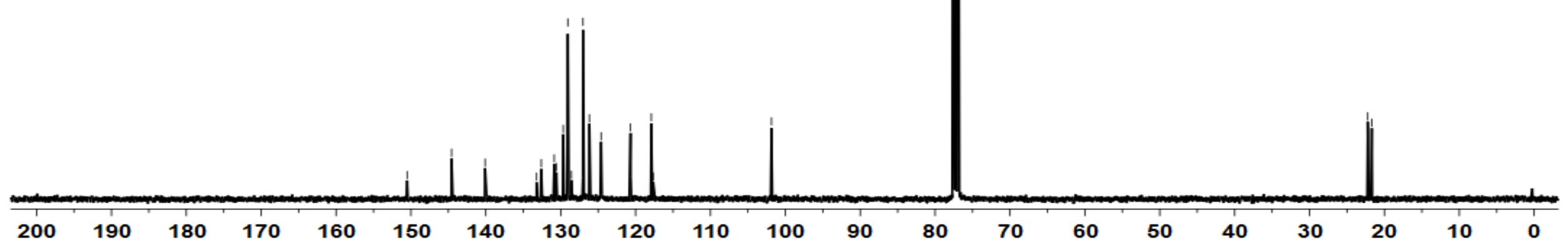




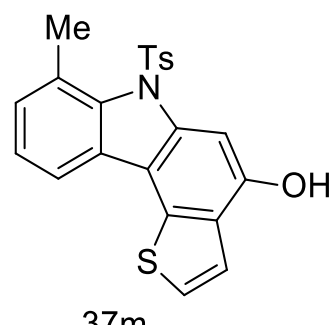

$100 \mathrm{MHz}, \mathrm{CDCl} 3$

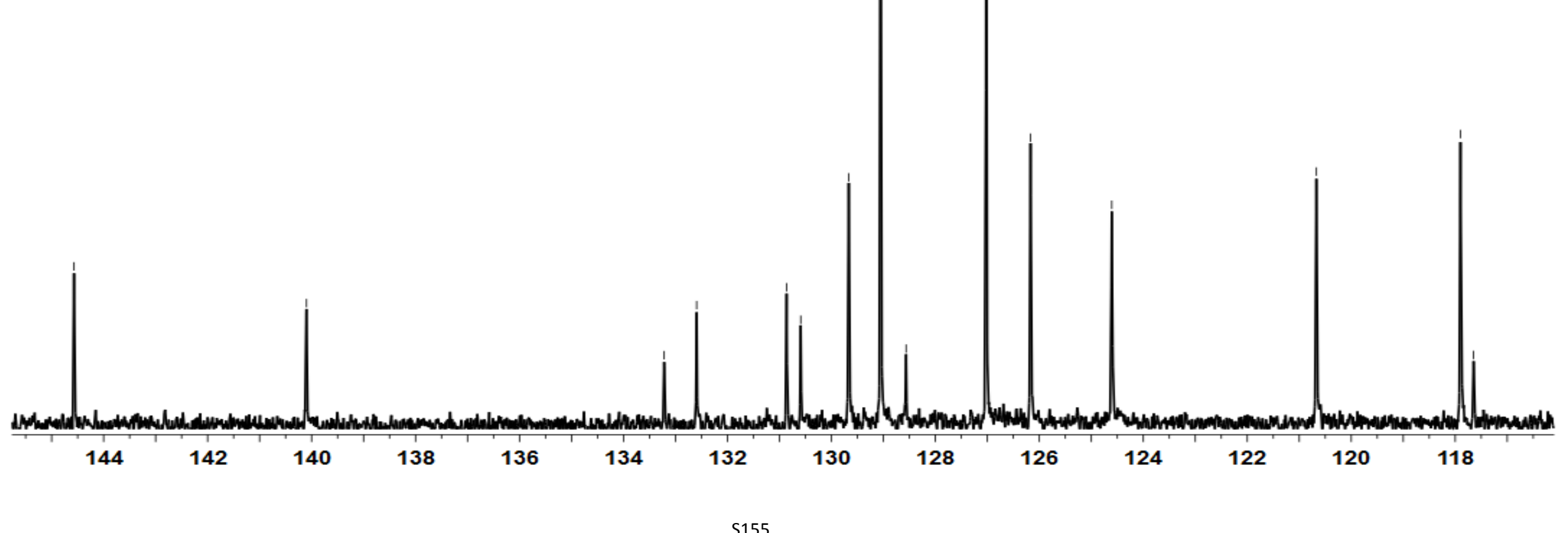




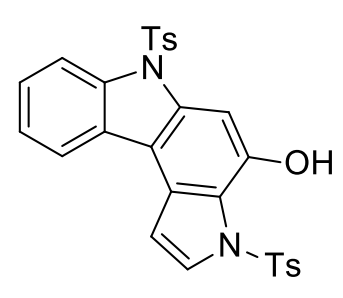

370

$500 \mathrm{MHz}, \mathrm{CDCl} 3$

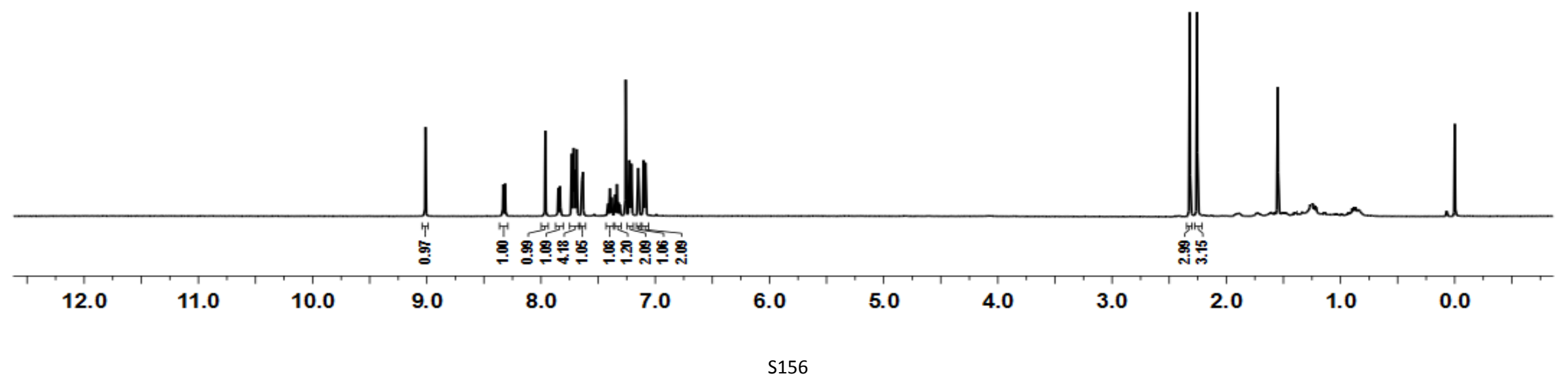




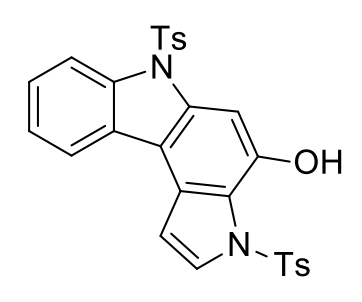

$500 \mathrm{MHz}, \mathrm{CDCl} 3$

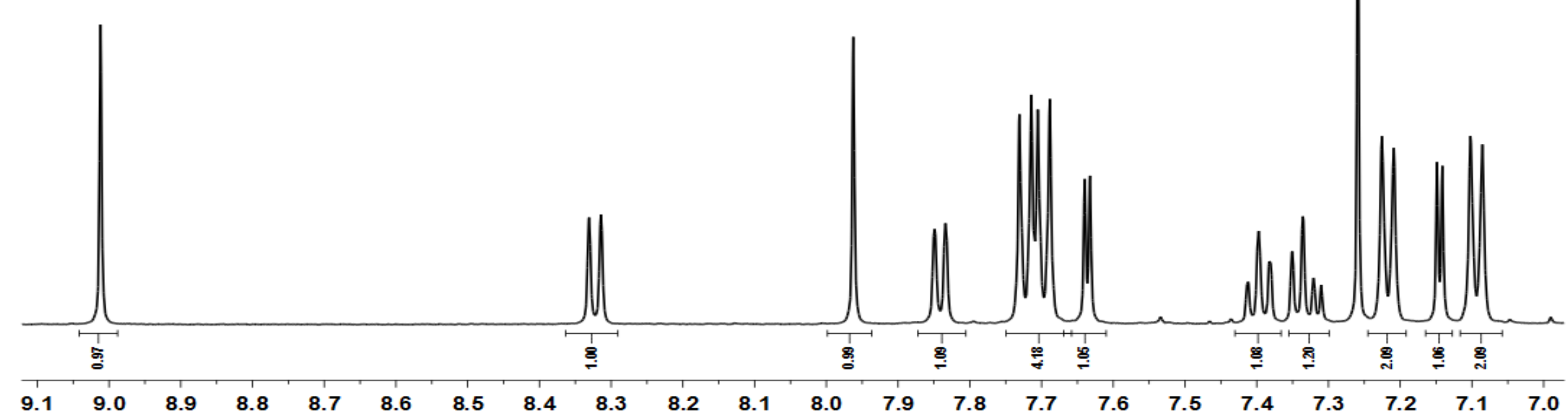



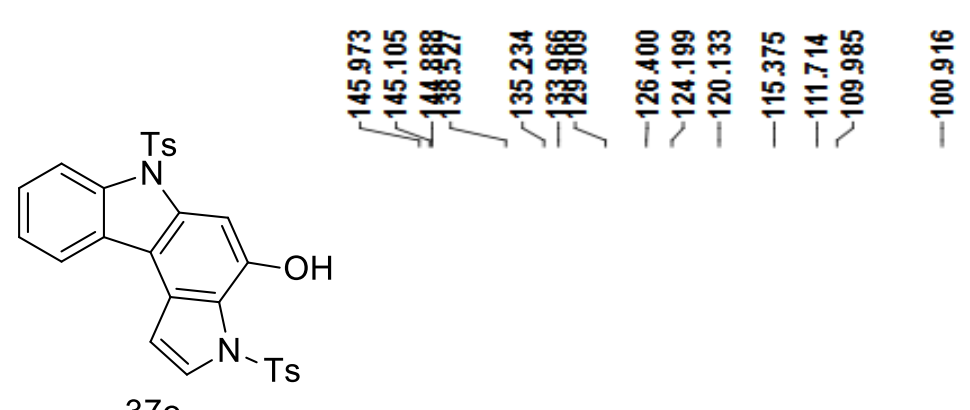

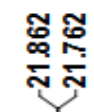

$100 \mathrm{MHz}, \mathrm{CDCl} 3$

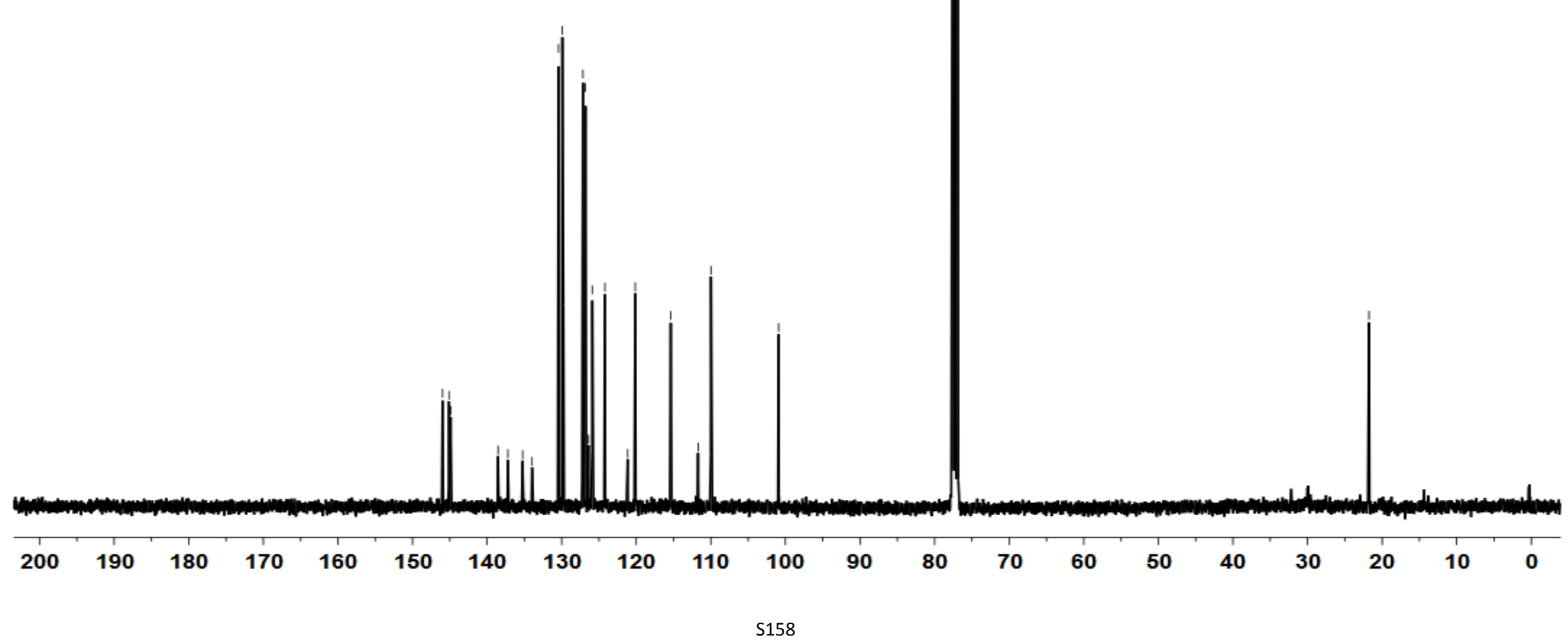




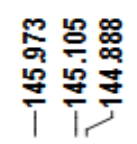

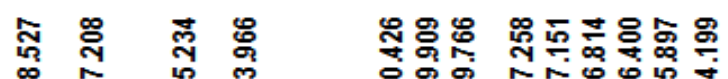

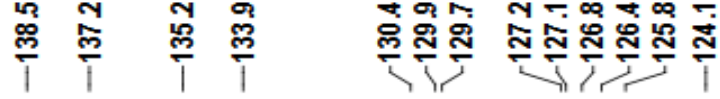

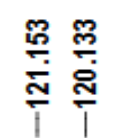

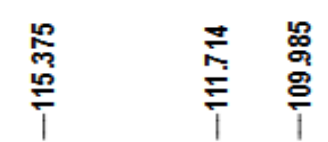

응

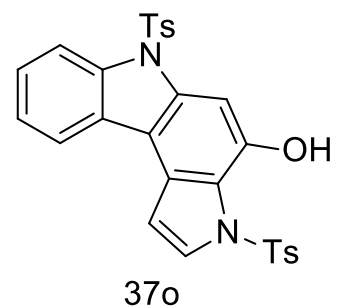

$100 \mathrm{MHz}, \mathrm{CDCl} 3$

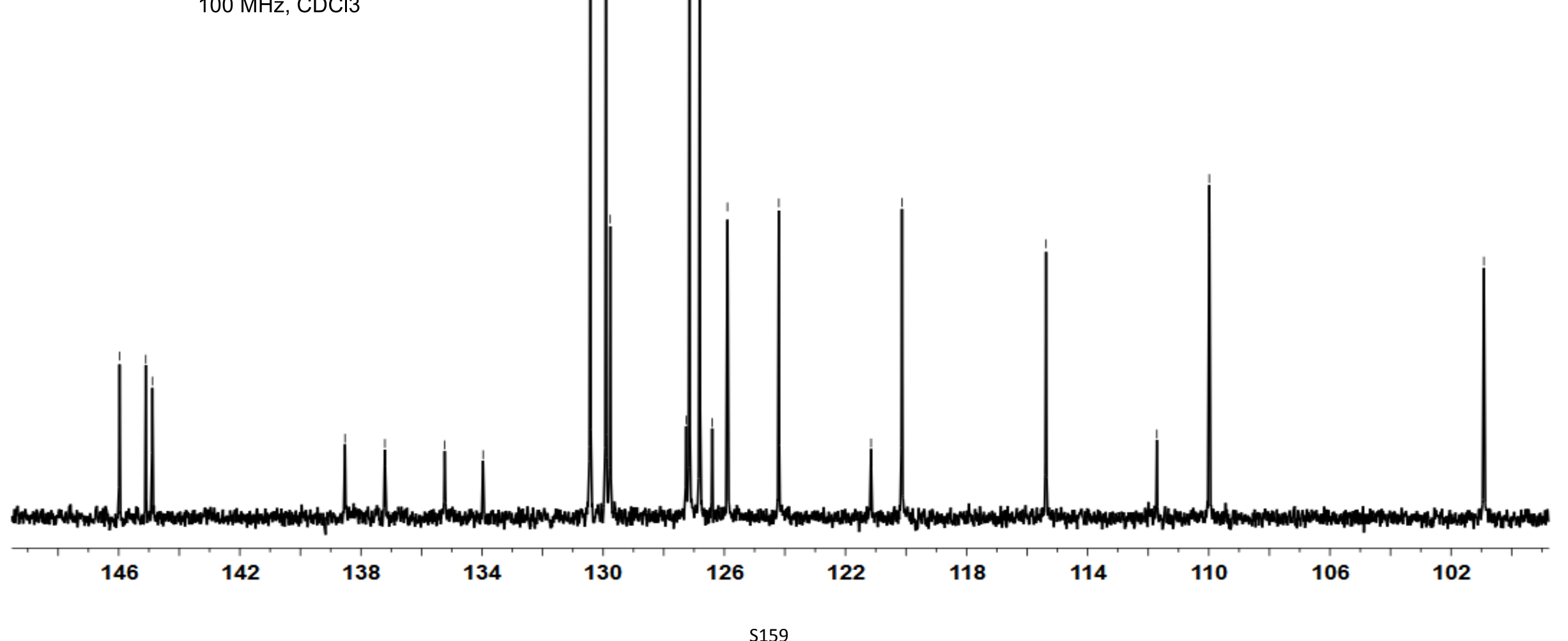




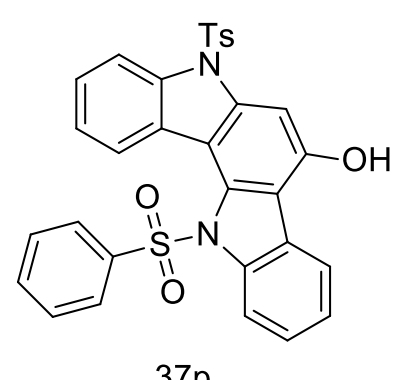

$37 p$

$500 \mathrm{MHz}, \mathrm{CDCl} 3$

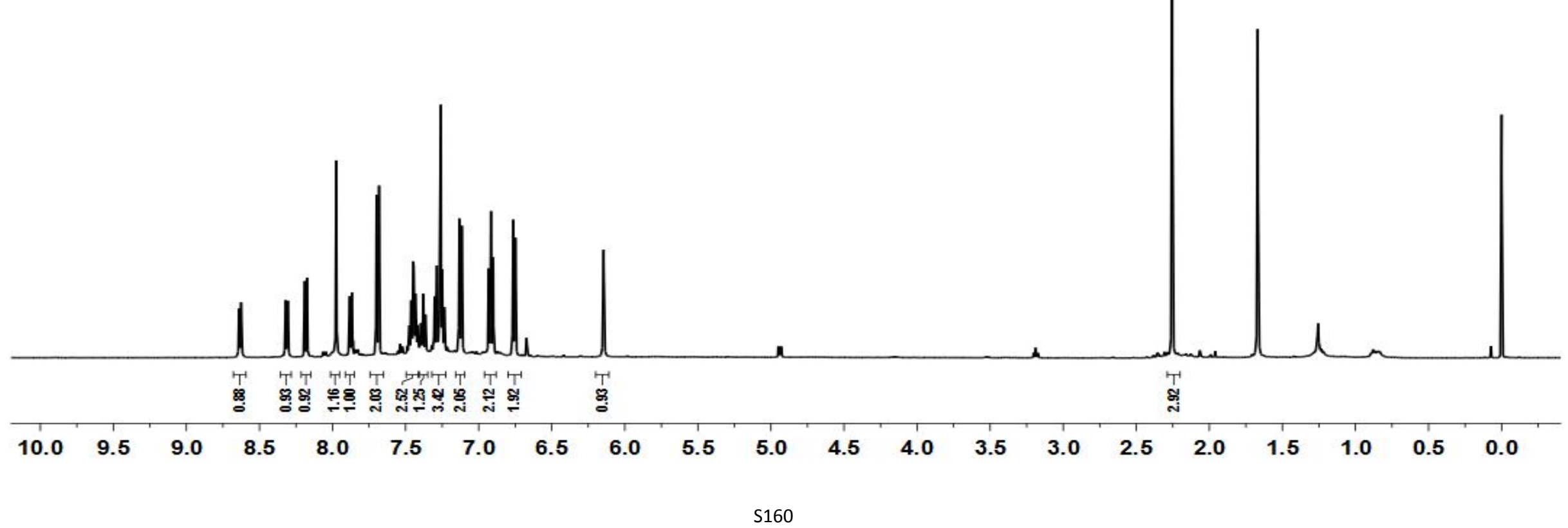




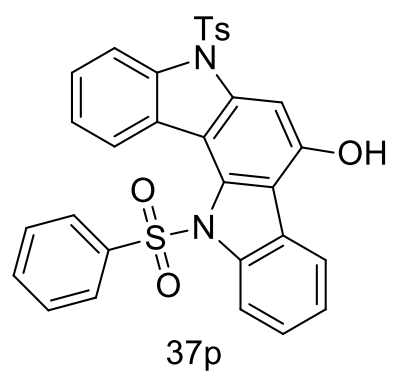

$125 \mathrm{MHz}, \mathrm{CDCl} 3$

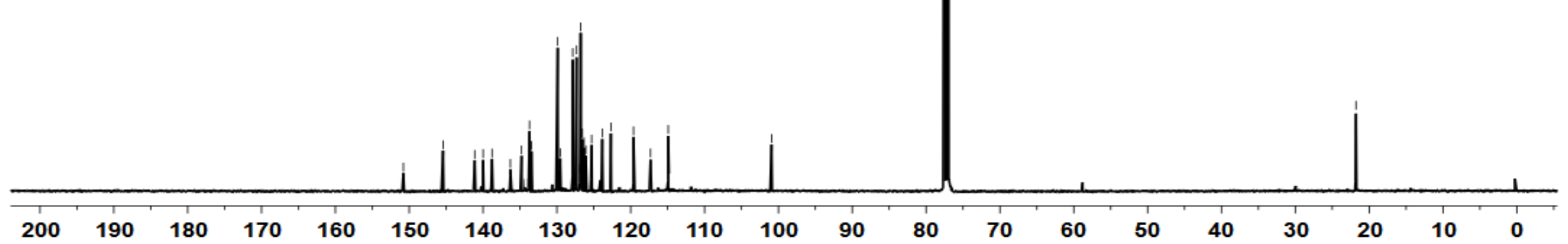




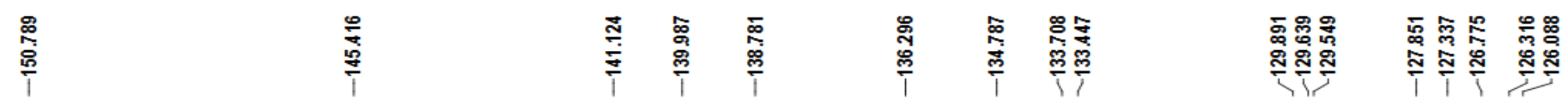

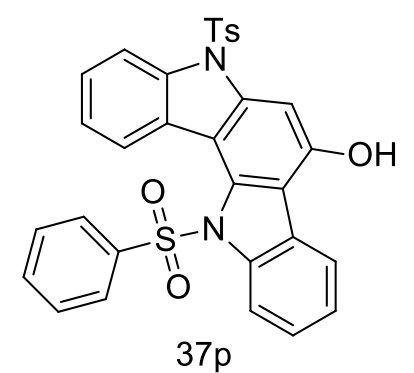

$125 \mathrm{MHz}, \mathrm{CDCl} 3$

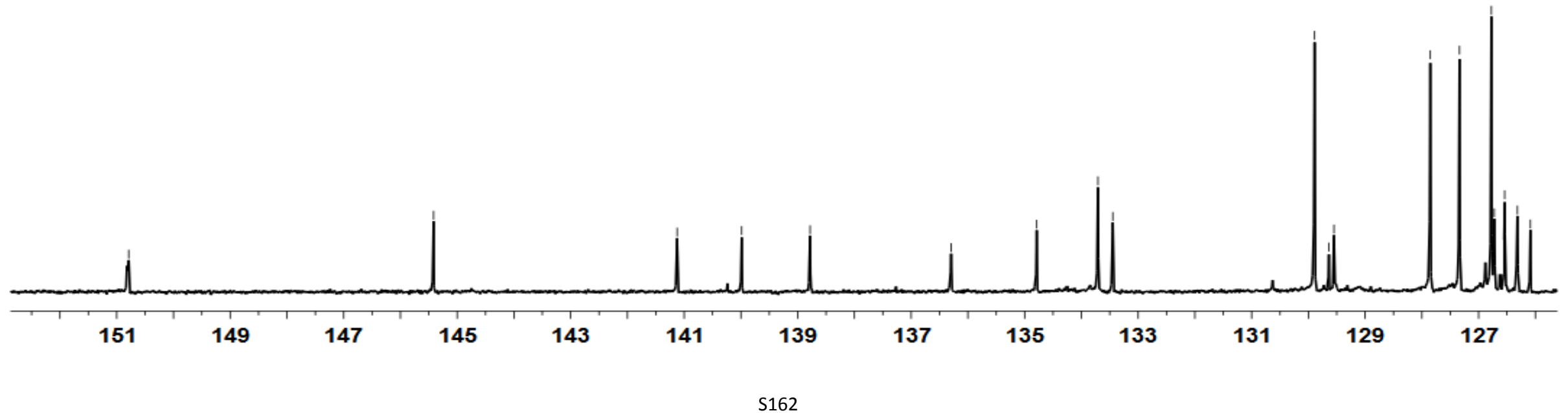




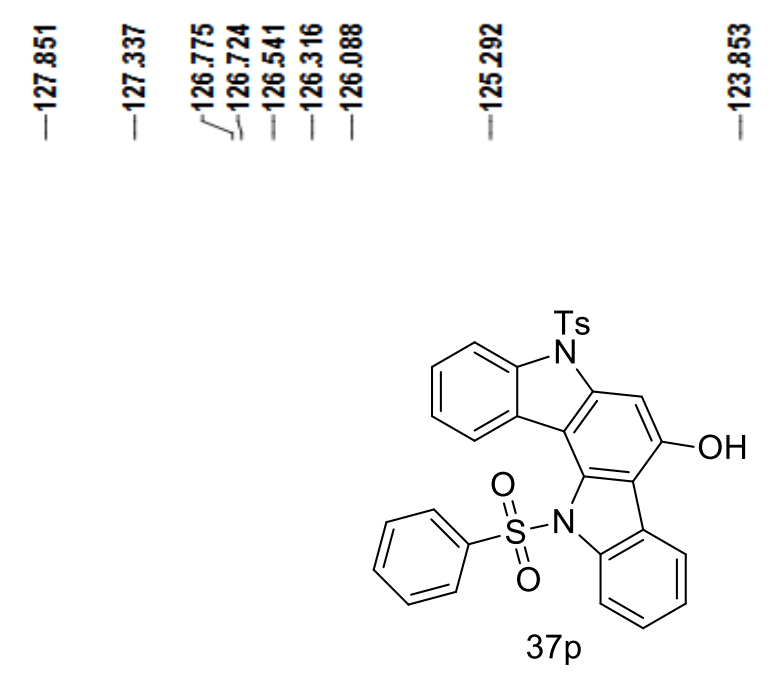

$125 \mathrm{MHz}, \mathrm{CDCl} 3$

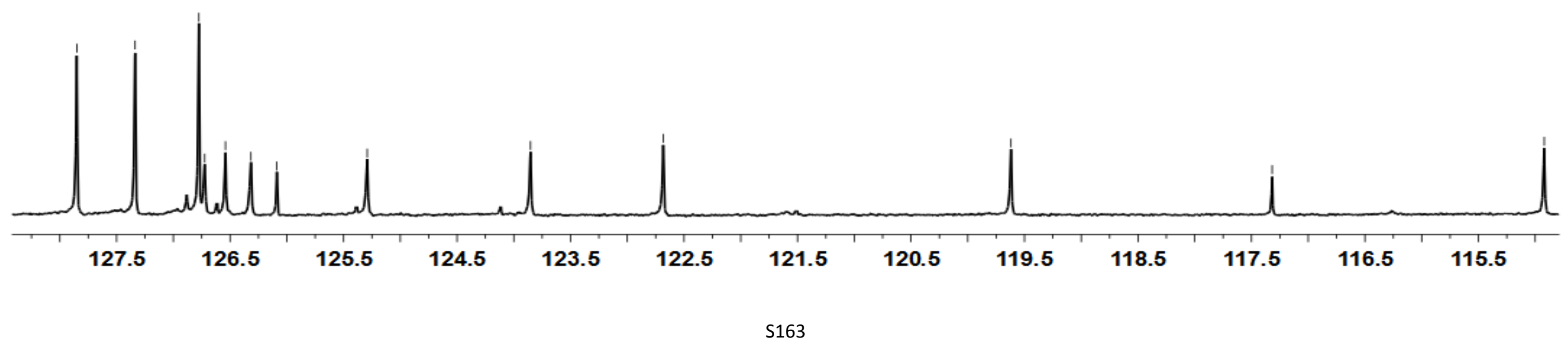




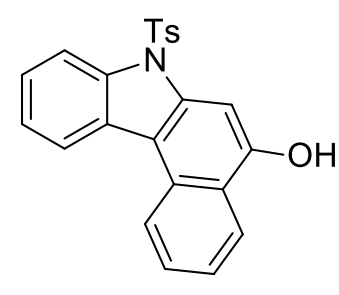

35

$500 \mathrm{MHz}$, (CD3)2CO

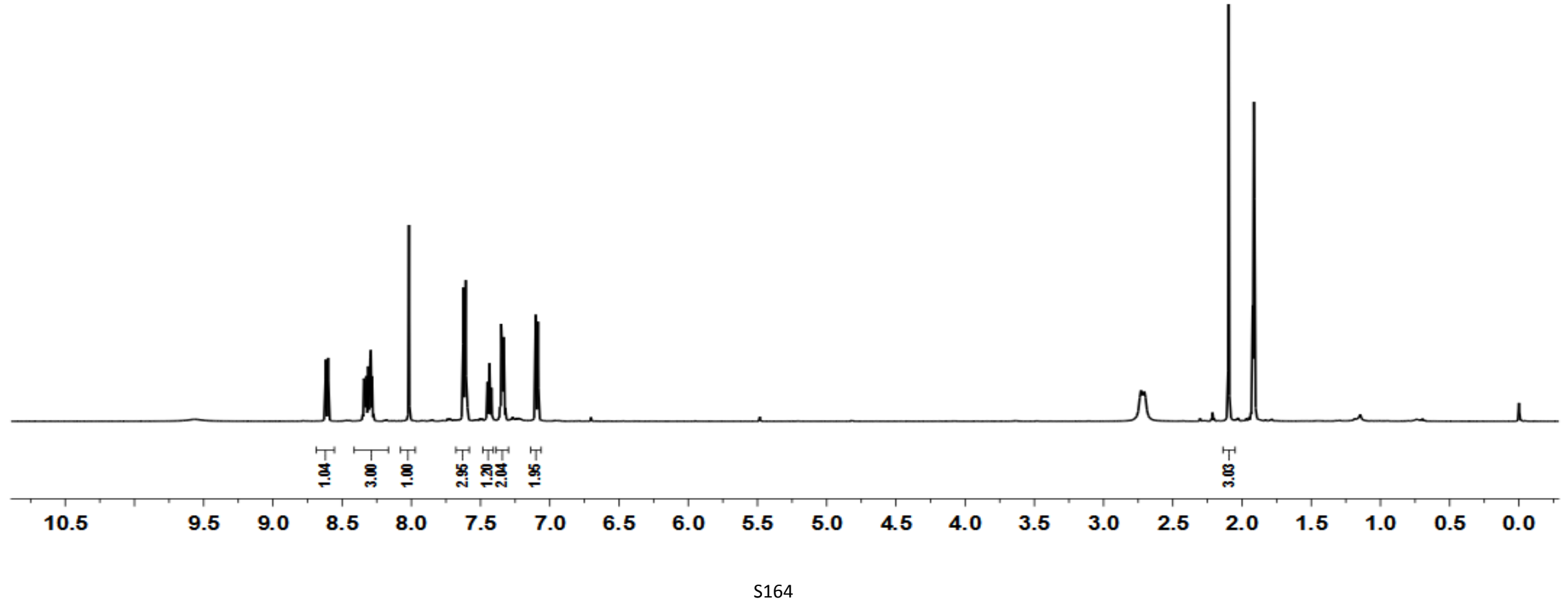




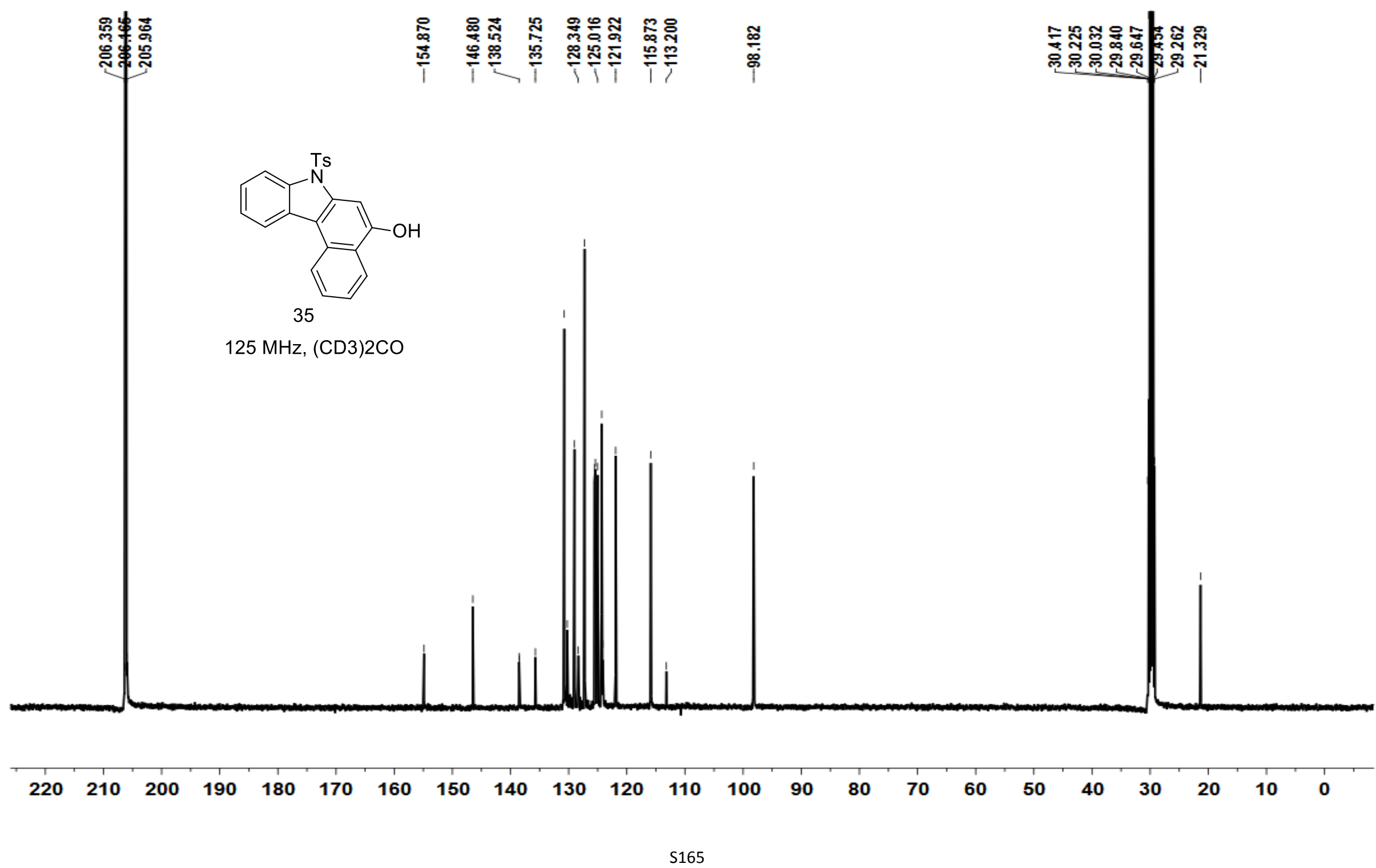




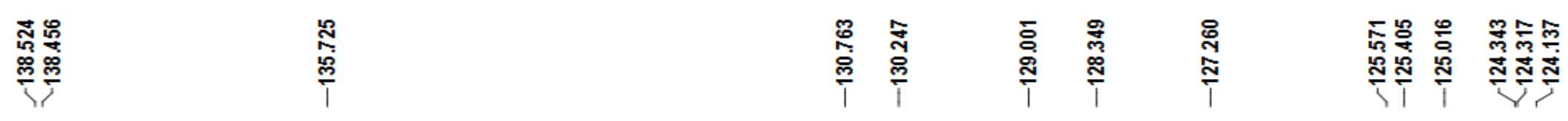

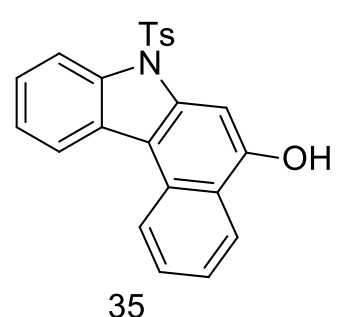

$125 \mathrm{MHz}$, (CD3)2CO

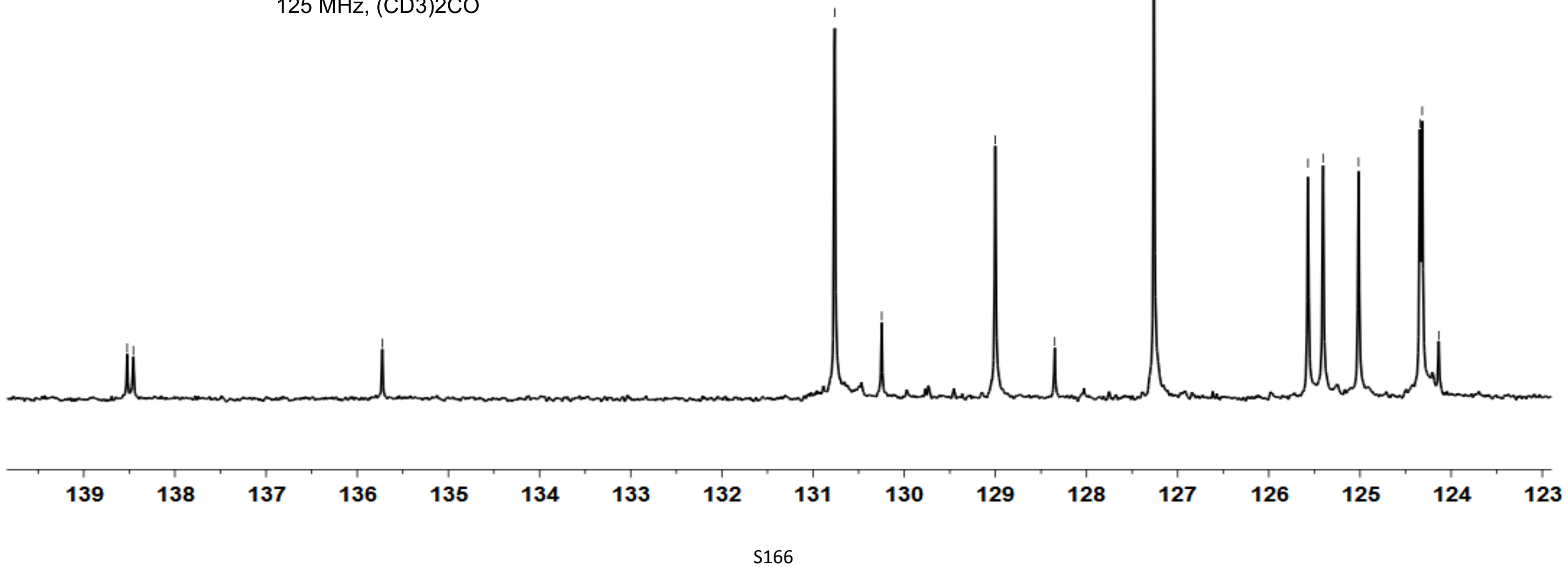

